\title{
Divergent Conversion of Double Isocyanides with Alkenyl Bromide to Polysubstituted Pyrroles and 4-Imino-4,5-Dihydropyrrolo[3,4-b]pyrrol-6(1H)-One Derivatives by Pd-Catalyzed Tandem Cyclization Reactions
}

Zhi-Lin Ren, ${ }^{\S, a}$ Ji-Ying Qiu, ${ }^{\S, a}$ Ling-Ling Yuan, ${ }^{\text {a }}$ Yue-Fei Yuan, ${ }^{a}$ Shuang Cai, ${ }^{\text {a }}$ Jun Li, ${ }^{a}$ Chi Kong, ${ }^{a}$ Ping $\mathrm{He}^{*}$, a and Long Wang ${ }^{*}, \mathrm{~b}$

${ }^{a}$ College of Chemical Engineering, Hubei University of Arts and Science, Xiangyang, 441053, Hubei Province, P. R. of China.

${ }^{\mathrm{b}}$ Key laboratory of inorganic nonmetallic crystalline and energy conversion materials, College of Materials and Chemical Engineering, China Three Gorges University, Yichang, Hubei Province, 443002, P. R.of China.

Email: pinghe129@163.com; wanglongchem@ctgu.edu.cn

\section{Contents}

\begin{tabular}{|c|l|c|}
\hline 1 & General Information & S2-S2 \\
\hline 2 & General Procedure for the Preparation of 4, 6 and 7 & S2-S3 \\
\hline 3 & X-ray Crystallography Data of 6f and 7f & S4-S7 \\
\hline 4 & HRMS Figures of 6f, 7f and 15a & S8 \\
\hline 5 & Characterization Datas & S9-S25 \\
\hline 6 & Copies of $^{1}$ H and ${ }^{13}$ C NMR Spectrum & S26-S84 \\
\hline
\end{tabular}




\section{General Information}

Unless otherwise noted, all reactions were performed in Schlenk tube by oil bath under an atmosphere of air balloon. Materials were purchased from commercial suppliers without further purification. All solvents were used without further distillation. Oil bath was used for all required reactions with magnetic stirring. Column chromatography purifications were performed under "flash" conditions using 200-300 mesh silica gel. Analytical TLC was carried out on silica gel 60 F254 plates which were visualized by exposure to ultraviolet light. Melting points were determined with an X-4 model apparatus. HRMS was measured on an FT-ICR MS Bruker15TSolarix spectrometer. NMR spectra were recorded on a Bruker 400 spectrometer calibrated to $\mathrm{CDCl}_{3}$ or DMSO- $d_{6}$ using tetramethylsilane (TMS) as internal standards. ${ }^{1} \mathrm{H}$ NMR spectral data are reported in terms of chemical shift $(\delta$, ppm), multiplicity $(\mathrm{s}=$ single, $\mathrm{d}=$ doublet, $\mathrm{t}=$ triplet, $\mathrm{q}=$ quartet, $\mathrm{m}=$ multiplet $)$, coupling constant $(\mathrm{Hz})$, and integration. ${ }^{13} \mathrm{C}$ NMR spectral data are reported in terms of chemical shift.

\section{General Procedure for the Preparation of 4, 6 and 7}

\section{General procedure for the preparation of compounds 4:}

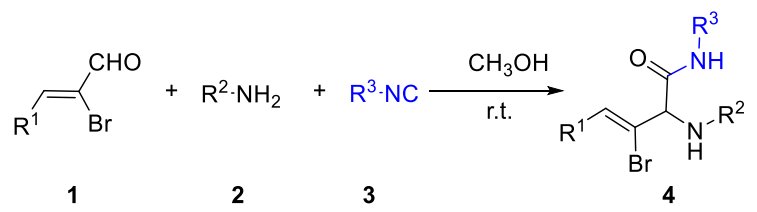

A mixture of $\alpha$-Bromocinnamaldehydes 1 ( $2 \mathrm{mmol})$, anilines 2 (2 mmol, 1.0 eqiuv.) and isocyanides 3 ( $2 \mathrm{mmol}, 1.0$ eqiuv.) were stirred in methanol (0.2 M, 10 $\mathrm{mL})$ in the presence of a catalytic amount of $\mathrm{H}_{3} \mathrm{PO}_{4}(20 \mathrm{~mol} \%, 0.04 \mathrm{mmol}, 0.2$ eqiuv.) at room temperature for $8 \mathrm{~h}$. The reaction mixture was then monitored by TLC. It should be noted that the Ugi reactions could not take place successfully when the substituents $\left(\mathrm{R}^{3}=4-\mathrm{Cl}, 4-\mathrm{Br}, 4-\mathrm{CH}_{3}\right)$ on the aromatic ring of isocyanides were used. After completion, the solvent was removed under reduced pressure and the residue was purified by flash chromatography on silica gel with ethylacetate/petroleum ether $(1: 15-20)$ as the eluent to give $\mathbf{4 a - z}$ in a yield of $47-84 \%$. 


\section{General procedure for the preparation of compounds 6:}

In an Schlenk tube with air balloon, substrates 4 (1.0 mmol), isocyanides 5 (2.4 mmol, 2.4 eqiuv.), $\mathrm{Pd}(\mathrm{OAc})_{2}$ (11 mg, $0.05 \mathrm{mmol} 0.05$ eqiuv.), ligand DPPB (21 mg, 0.05 mmol, 0.05 eqiuv.) and $\mathrm{Cs}_{2} \mathrm{CO}_{3}(652 \mathrm{mg}, 2.0 \mathrm{mmol}, 2.0$ eqiuv.) were stirred in 1,4-dioxane $(0.2 \mathrm{M}, 5 \mathrm{~mL})$ at $100{ }^{\circ} \mathrm{C}$ by oil bath for $5 \mathrm{~h}$. The mixtures were monitored by TLC. After completion of the reaction, the reaction mixtures were concentrated under reduced pressure and purified by silica flash chromatography (ethylacetate/petroleum ether $=1: 12-15, \mathrm{v} / \mathrm{v}$ ) to afford polysubstituted pyrroles derivatives 6a-v in $61-83 \%$ yields.

\section{General procedure for the preparation of compounds 7:}

In an Schlenk tube with air balloon, substrates $4(1.0 \mathrm{mmol})$, isocyanides 5 (2.4 mmol, 2.4 eqiuv.), $\mathrm{Pd}(\mathrm{OAc})_{2}(11 \mathrm{mg}, 0.05 \mathrm{mmol}, 0.05$ eqiuv.), ligand $\mathrm{DPPB}$ (21 mg, 0.05 mmol, 0.05 eqiuv.) and $\mathrm{Cs}_{2} \mathrm{CO}_{3}(652 \mathrm{mg}, 2.0 \mathrm{mmol}, 2.0$ eqiuv.) were stirred in 1,4-dioxane $(0.2 \mathrm{M}, 5 \mathrm{~mL})$ at $100{ }^{\circ} \mathrm{C}$ by oil bath for $5 \mathrm{~h}$. The mixtures were monitored by TLC. After completion of the reaction, the reaction mixtures were concentrated under reduced pressure and purified by silica flash chromatography (ethylacetate/petroleum ether $=1$ : 25-30, v/v) to afford 4-imino-4,5-dihydropyrrolo[3,4-b]pyrrol-6(1H)-one derivatives 7a-k in $75-86 \%$ yields.

\section{General procedure of scale-up synthesis of $7 \mathrm{~b}$ :}

In an Schlenk tube with air balloon, substrate 4i $(1.34 \mathrm{~g}, 3.0 \mathrm{mmol})$, isocyanide $5 \mathbf{b}$ (0.60 g, $7.2 \mathrm{mmol}, 2.4$ eqiuv.), $\mathrm{Pd}(\mathrm{OAc})_{2}$ (33 mg, $0.15 \mathrm{mmol}, 0.05$ eqiuv.), ligand DPPB (63 mg, 0.15 mmol, 0.05 eqiuv.) and $\mathrm{Cs}_{2} \mathrm{CO}_{3}$ (1.96 g, $6.0 \mathrm{mmol}, 2.0$ eqiuv.) were stirred in 1,4-dioxane $(0.2 \mathrm{M}, 15 \mathrm{~mL})$ at $100{ }^{\circ} \mathrm{C}$ by oil bath for $5 \mathrm{~h}$. The mixture was monitored by TLC. After completion of the reaction, the reaction mixture was concentrated under reduced pressure and purified by silica flash chromatography (ethylacetate/petroleum ether $=1: 30, \quad \mathrm{v} / \mathrm{v})$ to afford 4-imino-4,5-dihydropyrrolo[3,4-b]pyrrol-6(1H)-one derivatives $7 \mathbf{b}$ in $77 \%$ yield. 


\section{X-ray Crystallography Data of $6 f$ and $7 f$}

Crystal sample preparation of $\mathbf{6 f}$ : A solution of compound $\mathbf{6 f}(0.02 \mathrm{~g})$ in AcOEt (1.5 $\mathrm{mL})$ was placed in a vial $(10 \mathrm{~mL})$. Then $n$-hexane $(4 \mathrm{~mL})$ was added to the solution with a dropper. The single crystal 6f was obtained by slowly evaporating mixed solvent at room temperature under the air conditions. A suitable crystal was selected and tested on a XtaLAB AFC12 (RINC): Kappa single diffractometer. The crystal was kept at 293(2) K during data collection. Using Olex2 [1], the structure was solved with the ShelXT [2] structure solution program using Intrinsic Phasing and refined with the ShelXL [3] refinement package using Least Squares minimisation.

1. Dolomanov, O.V., Bourhis, L.J., Gildea, R.J, Howard, J.A.K. \& Puschmann, H. (2009), J. Appl. Cryst. 42, 339-341.

2. Sheldrick, G.M. (2015). Acta Cryst. A71, 3-8.

3. Sheldrick, G.M. (2015). Acta Cryst. C71, 3-8.

\section{Crystal structure determination of [exp_1889]}

Crystal Data for $\mathrm{C}_{30} \mathrm{H}_{40} \mathrm{~N}_{4} \mathrm{O}_{2}(\mathrm{M}=488.32 \mathrm{~g} / \mathrm{mol})$ : monoclinic, space group $\mathrm{P} 2_{1} / \mathrm{c}$ (no. 14), $a=12.0603(3) \AA, b=20.6826(4) \AA, c=12.3915(3) \AA, \beta=111.379(2)^{\circ}, V=$ 2878.23(12) $\AA^{3}, \mathrm{Z}=4, \mathrm{~T}=293(2) \mathrm{K}, \mu(\mathrm{CuK \alpha})=0.556 \mathrm{~mm}^{-1}$, Dcalc $=1.035 \mathrm{~g} / \mathrm{cm}^{3}$, 17355 reflections measured $\left(7.872^{\circ} \leqslant 2 \Theta \leqslant 148.794^{\circ}\right), 5702$ unique $\left(\mathrm{R}_{\text {int }}=\right.$ $\left.0.0919, R_{\text {sigma }}=0.0668\right)$ which were used in all calculations. The final $R_{1}$ was 0.0812 $(\mathrm{I}>2 \sigma(\mathrm{I}))$ and $\mathrm{wR}_{2}$ was 0.2442 (all data).

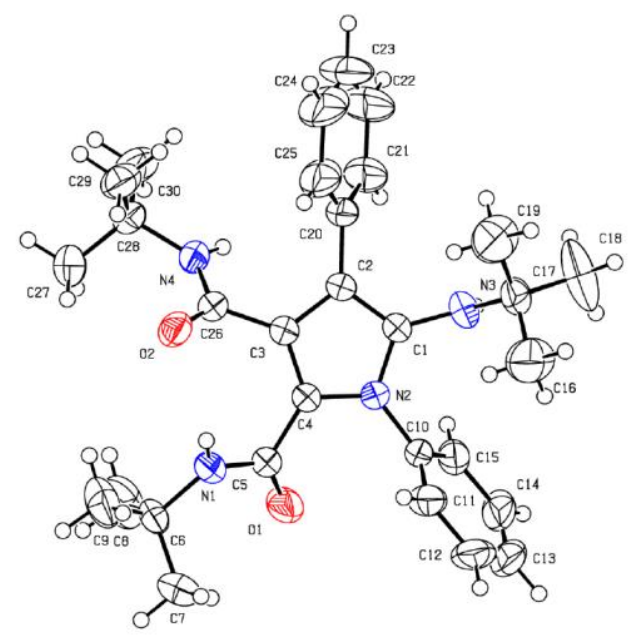

Figure S1. X-ray crystal structure of $\mathbf{6} \mathbf{f}$ is drawn at the $50 \%$ probability. 
Table 1 Crystal data and structure refinement for exp_1889.

\begin{tabular}{|c|c|}
\hline Identification code & exp_1889 \\
\hline Empirical formula & $\mathrm{C}_{30} \mathrm{H}_{40} \mathrm{~N}_{4} \mathrm{O}_{2}$ \\
\hline Formula weight & 488.32 \\
\hline Temperature/K & 293(2) \\
\hline Crystal system & monoclinic \\
\hline Space group & $\mathrm{P} 2{ }_{1} / \mathrm{c}$ \\
\hline $\mathrm{a} / \AA$ & $12.0603(3)$ \\
\hline $\mathrm{b} / \mathrm{A}$ & $20.6826(4)$ \\
\hline $\mathrm{c} / \AA$ & $12.3915(3)$ \\
\hline $\mathrm{a} /{ }^{\circ}$ & 90 \\
\hline$\beta /^{\circ}$ & $111.379(2)$ \\
\hline$\gamma /^{\circ}$ & 90 \\
\hline Volume $/ \AA^{3}$ & $2878.23(12)$ \\
\hline $\mathrm{Z}$ & 4 \\
\hline$\rho_{\text {calc }} g / \mathrm{cm}^{3}$ & 1.035 \\
\hline$\mu / \mathrm{mm}^{-1}$ & 0.556 \\
\hline $\mathrm{F}(000)$ & 897.0 \\
\hline Crystal size $/ \mathrm{mm}^{3}$ & $? \times ? \times ?$ \\
\hline Radiation & $\mathrm{CuK} a \quad(\lambda=1.54184)$ \\
\hline 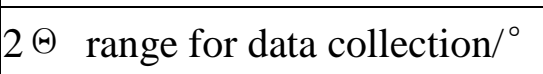 & 7.872 to 148.794 \\
\hline Index ranges & $-15 \leqslant \mathrm{~h} \leqslant 14,-25 \leqslant \mathrm{k} \leqslant 25,-9 \leqslant 1 \leqslant 14$ \\
\hline Reflections collected & 17355 \\
\hline Independent reflections & $5702\left[\mathrm{R}_{\mathrm{int}}=0.0919, \mathrm{R}_{\mathrm{sigma}}=0.0668\right]$ \\
\hline Data/restraints/parameters & $5702 / 0 / 334$ \\
\hline Goodness-of-fit on $\mathrm{F}^{2}$ & 1.064 \\
\hline Final $R$ indexes $[\mathrm{I}>=2 \sigma \quad(\mathrm{I})]$ & $\mathrm{R}_{1}=0.0812, \mathrm{wR}_{2}=0.2324$ \\
\hline Final $\mathrm{R}$ indexes [all data] & $\mathrm{R}_{1}=0.0897, \mathrm{wR}_{2}=0.2442$ \\
\hline Largest diff. peak/hole / e $\AA^{-3}$ & $0.42 /-0.44$ \\
\hline
\end{tabular}


Crystal sample preparation of 7f: A solution of compound 7f (0.02 g) in Petroleum ether $(5 \mathrm{~mL})$ was placed in a vial $(10 \mathrm{~mL})$. The single crystal $7 \mathbf{f}$ was obtained by slowly evaporating mixed solvent at room temperature under the air conditions. A suitable crystal was selected and tested on a XtaLAB AFC12 (RINC): Kappa single diffractometer. The crystal was kept at 293(2) K during data collection. Using Olex2 [1], the structure was solved with the SHELXT [2] structure solution program using Intrinsic Phasing and refined with the SHELXL [3] refinement package using Least Squares minimisation.

1. Dolomanov, O.V., Bourhis, L.J., Gildea, R.J, Howard, J.A.K. \& Puschmann, H. (2009), J. Appl. Cryst. 42, 339-341.

2. Sheldrick, G.M. (2015). Acta Cryst. A71, 3-8.

3. Sheldrick, G.M. (2015). Acta Cryst. C71, 3-8.

\section{Crystal structure determination of [exp_2327]}

Crystal Data for $\mathrm{C}_{32} \mathrm{H}_{38} \mathrm{Cl}_{2} \mathrm{~N}_{4} \mathrm{O}(\mathrm{M}=564.24 \mathrm{~g} / \mathrm{mol})$ : monoclinic, space group $\mathrm{P} 2_{1}$ (no. 4), $\mathrm{a}=10.9778(2) \AA, \mathrm{b}=9.8823(2) \AA, \mathrm{c}=14.2719(2) \AA, \beta=93.2770(10)^{\circ}, \mathrm{V}=$ 1545.77(5) $\AA^{3}, \mathrm{Z}=2, \mathrm{~T}=293(2) \mathrm{K}, \mu(\mathrm{Cu} \mathrm{K} \alpha)=2.119 \mathrm{~mm}^{-1}$, Dcalc $=1.217 \mathrm{~g} / \mathrm{cm}^{3}$, 8470 reflections measured $\left(8.068^{\circ} \leqslant 2 \Theta \leqslant 148.258^{\circ}\right), 4671$ unique $\left(\mathrm{R}_{\text {int }}=\right.$ 0.0297, $R_{\text {sigma }}=0.0407$ ) which were used in all calculations. The final $R_{1}$ was 0.0402 $(\mathrm{I}>2 \sigma(\mathrm{I}))$ and $\mathrm{wR}_{2}$ was 0.1167 (all data).

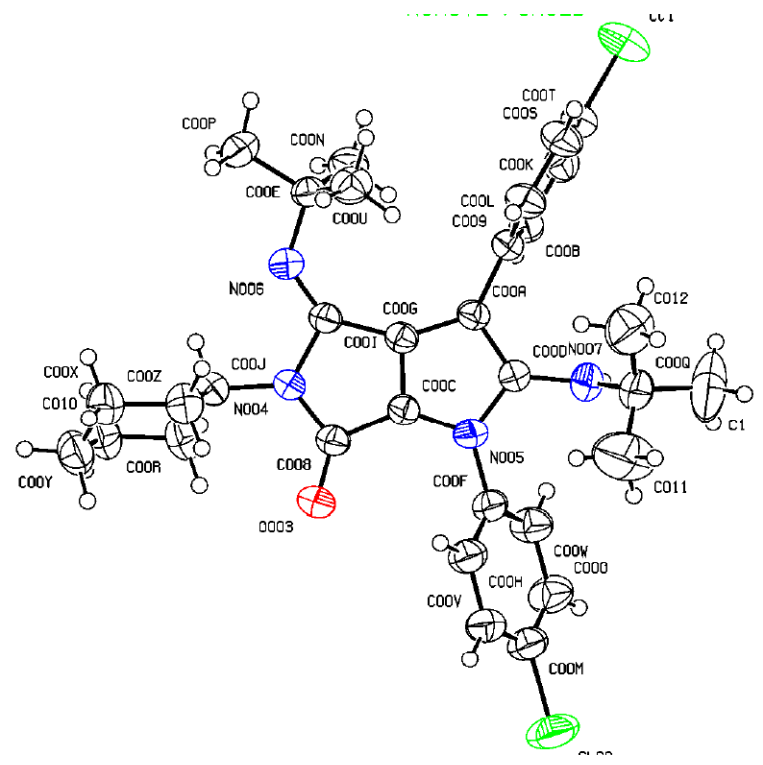

Figure S2. X-ray crystal structure of $\mathbf{7 f}$ is drawn at the 50\% probability. 
Table 2 Crystal data and structure refinement for exp_2327.

\begin{tabular}{|c|c|}
\hline Identification code & exp_2327 \\
\hline Empirical formula & $\mathrm{C}_{32} \mathrm{H}_{38} \mathrm{Cl}_{2} \mathrm{~N}_{4} \mathrm{O}$ \\
\hline Formula weight & 564.24 \\
\hline Temperature/K & 293(2) \\
\hline Crystal system & monoclinic \\
\hline Space group & $\mathrm{P} 2_{1}$ \\
\hline $\mathrm{a} / \AA$ & $10.9778(2)$ \\
\hline $\mathrm{b} / \AA ̊$ & $9.8823(2)$ \\
\hline $\mathrm{c} / \AA ̊ \AA$ & $14.2719(2)$ \\
\hline$\alpha /{ }^{\circ}$ & 90 \\
\hline$\beta /^{\circ}$ & $93.2770(10)$ \\
\hline$\gamma /^{\circ}$ & 90 \\
\hline Volume $/ \AA^{3}$ & $1545.77(5)$ \\
\hline $\mathrm{Z}$ & 2 \\
\hline$\rho_{\text {calc }} / \mathrm{cm}^{3}$ & 1.217 \\
\hline$\mu / \mathrm{mm}^{-1}$ & 2.119 \\
\hline $\mathrm{F}(000)$ & 602.0 \\
\hline Crystal size $/ \mathrm{mm}^{3}$ & $0.2 \times 0.15 \times 0.1$ \\
\hline Radiation & $\mathrm{Cu} \mathrm{K} \mathrm{a} \quad(\lambda=1.54184)$ \\
\hline $2 \Theta$ range for data collection $/^{\circ}$ & 8.068 to 148.258 \\
\hline Index ranges & $-8 \leqslant \mathrm{~h} \leqslant 13,-12 \leqslant \mathrm{k} \leqslant 9,-17 \leqslant 1 \leqslant 17$ \\
\hline Reflections collected & 8470 \\
\hline Independent reflections & $4671\left[R_{\text {int }}=0.0297, R_{\text {sigma }}=0.0407\right]$ \\
\hline Data/restraints/parameters & $4671 / 1 / 358$ \\
\hline Goodness-of-fit on $\mathrm{F}^{2}$ & 1.070 \\
\hline Final $R$ indexes $[I>=2 \sigma \quad(\mathrm{I})]$ & $\mathrm{R}_{1}=0.0402, \mathrm{wR}_{2}=0.1132$ \\
\hline Final $\mathrm{R}$ indexes [all data] & $\mathrm{R}_{1}=0.0434, \mathrm{wR}_{2}=0.1167$ \\
\hline Largest diff. peak/hole / e $\AA^{-3}$ & $0.27 /-0.22$ \\
\hline Flack parameter & $0.005(12)$ \\
\hline
\end{tabular}


4. HRMS Figures of $6 f, 7 f$ and $15 a$.

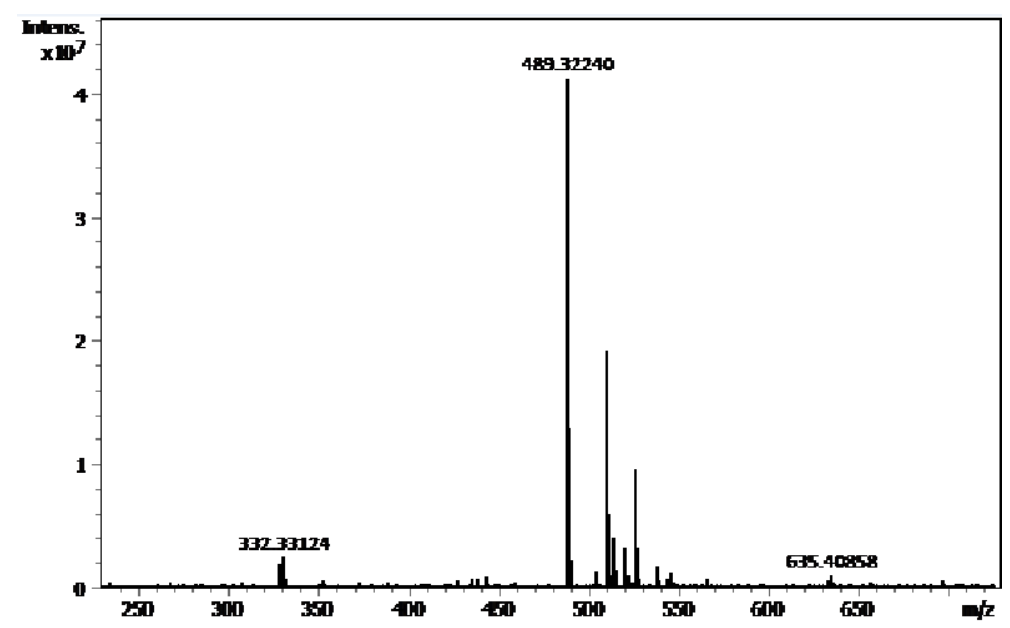

HRMS Figure of $\mathbf{6 f}$

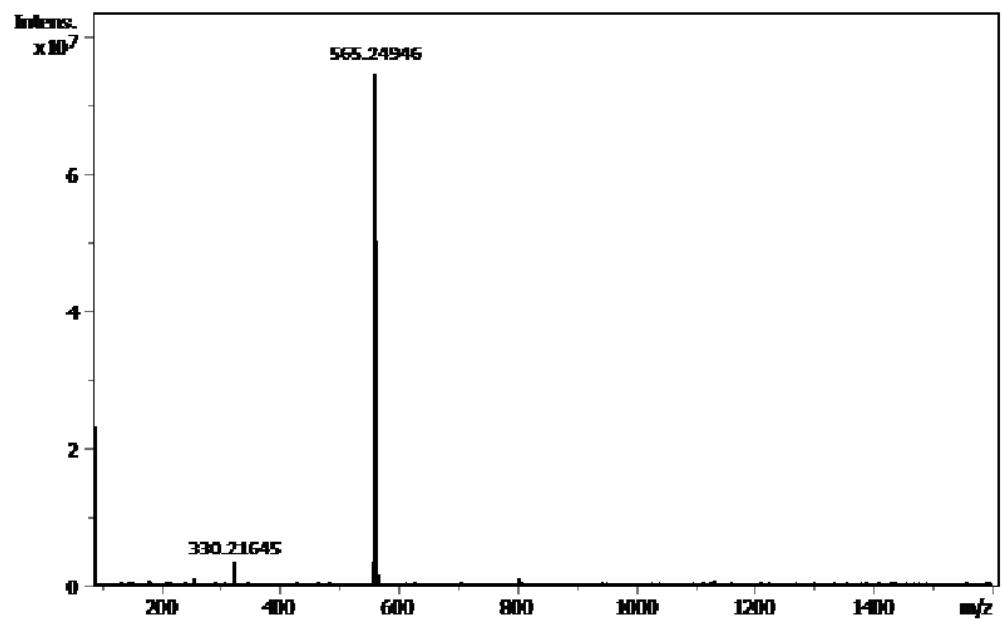

HRMS Figure of $7 \mathbf{f}$

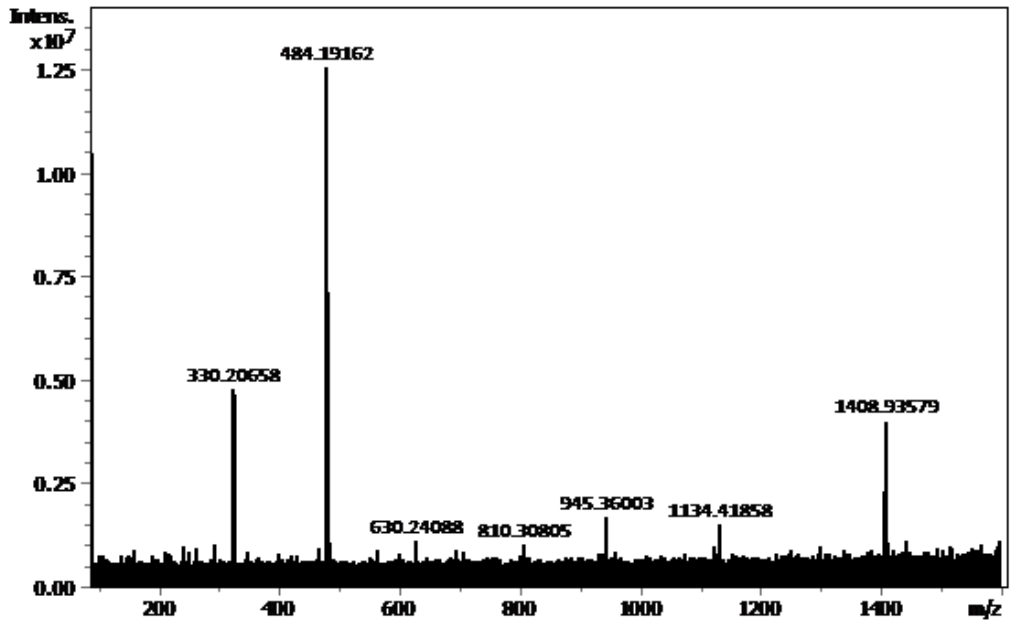

HRMS Figure of $\mathbf{1 5 a}$ 


\section{Characterization Datas}

(Z)-3-Bromo-2-((4-bromophenyl)amino)- $N$-(tert-butyl)-4-phenylbut-3-enamide (4a):

White solid, yield: $0.698 \mathrm{~g}(75 \%)$, ethylacetate/petroleum ether $=1: 20, \mathrm{mp} 138$ $140{ }^{\circ} \mathrm{C} .{ }^{1} \mathrm{H}$ NMR $\left(\mathrm{CDCl}_{3}, 400 \mathrm{MHz}\right) \delta(\mathrm{ppm})$ 7.58-6.53 (m, 10H), 6.20 (s, 1H), 5.09 $(\mathrm{s}, 1 \mathrm{H}), 4.48(\mathrm{~s}, 1 \mathrm{H}), 1.38(\mathrm{~s}, 9 \mathrm{H}) ;{ }^{13} \mathrm{C}\left\{{ }^{1} \mathrm{H}\right\} \mathrm{NMR}\left(\mathrm{CDCl}_{3}, 100 \mathrm{MHz}\right) \delta(\mathrm{ppm}) 166.8$, $144.5,134.5,132.0,131.7,129.1,128.6,128.2,122.8,115.5,110.6,63.7,51.9,28.5$; HRMS (ESI-TOF) m/z: $[\mathrm{M}+\mathrm{H}]^{+}$Calcd for $\mathrm{C}_{20} \mathrm{H}_{23} \mathrm{Br}_{2} \mathrm{~N}_{2} \mathrm{O}$ 465.0172; Found: 465.0171 .

(Z)-3-Bromo- $N$-(tert-butyl)-2-((3-chlorophenyl)amino)-4-phenylbut-3-enamide (4b):

White solid, yield: $0.657 \mathrm{~g}(78 \%)$, ethylacetate/petroleum ether $=1: 16, \mathrm{mp} 119$ $121{ }^{\circ} \mathrm{C} .{ }^{1} \mathrm{H}$ NMR $\left(\mathrm{CDCl}_{3}, 400 \mathrm{MHz}\right) \delta(\mathrm{ppm})$ 7.60-6.58 (m, 10H), $6.26(\mathrm{~s}, 1 \mathrm{H}), 5.72$ $(\mathrm{d}, J=4.8 \mathrm{~Hz}, 1 \mathrm{H}), 4.55(\mathrm{~d}, J=5.2 \mathrm{~Hz}, 1 \mathrm{H}), 1.39(\mathrm{~s}, 9 \mathrm{H}) ;{ }^{13} \mathrm{C}\left\{{ }^{1} \mathrm{H}\right\} \mathrm{NMR}\left(\mathrm{CDCl}_{3}\right.$, $100 \mathrm{MHz}) \delta(\mathrm{ppm}) 166.8,141.7,134.6,131.6,129.4,129.1,128.6,128.2,127.8$, 122.4, 120.3, 119.0, 112.8, 67.3, 51.9, 28.6; HRMS (ESI-TOF) m/z: $[\mathrm{M}+\mathrm{H}]^{+}$Calcd for $\mathrm{C}_{20} \mathrm{H}_{23} \mathrm{BrClN}_{2} \mathrm{O}$ 421.0677; Found: 421.0676 .

(Z)-3-Bromo- $N$-(tert-butyl)-4-phenyl-2-((4-(trifluoromethyl)phenyl)amino)but-3enamide (4c):

White solid, yield: $0.637 \mathrm{~g}(70 \%)$, ethylacetate/petroleum ether $=1: 20, \mathrm{mp} \mathrm{144-}$ $145{ }^{\circ} \mathrm{C} .{ }^{1} \mathrm{H}$ NMR $\left(\mathrm{CDCl}_{3}, 400 \mathrm{MHz}\right) \delta(\mathrm{ppm})$ 7.59-6.53 (m, 10H), 6.18 (s, 1H), 5.08 $(\mathrm{d}, J=4.4 \mathrm{~Hz}, 1 \mathrm{H}), 4.47(\mathrm{~d}, J=4.8 \mathrm{~Hz}, 1 \mathrm{H}), 1.38(\mathrm{~s}, 9 \mathrm{H}) ;{ }^{13} \mathrm{C}\left\{{ }^{1} \mathrm{H}\right\} \mathrm{NMR}\left(\mathrm{CDCl}_{3}\right.$, $100 \mathrm{MHz}) \delta(\mathrm{ppm}) 166.8,144.5,134.5,132.1,131.7,129.1,128.6,128.2,122.8$, 115.5, 110.7, 67.3, 52.0, 28.5; HRMS (ESI-TOF) m/z: $[\mathrm{M}+\mathrm{H}]^{+}$Calcd for $\mathrm{C}_{21} \mathrm{H}_{23} \mathrm{BrF}_{3} \mathrm{~N}_{2} \mathrm{O}$ 455.0940; Found: 455.0939 .

(Z)-3-Bromo- $N$-(tert-butyl)-4-phenyl-2-(phenylamino)but-3-enamide (4d):

White solid, yield: $0.565 \mathrm{~g}(73 \%)$, ethylacetate/petroleum ether $=1: 15, \mathrm{mp} 103-$ $104{ }^{\circ} \mathrm{C} .{ }^{1} \mathrm{H}$ NMR $\left(\mathrm{CDCl}_{3}, 400 \mathrm{MHz}\right) \delta(\mathrm{ppm})$ 7.59-6.67 (m, 11H), 6.39 (s, 1H), 4.92 (s, 1H), $4.53(\mathrm{~s}, 1 \mathrm{H}), 1.38(\mathrm{~s}, 9 \mathrm{H}) ;{ }^{13} \mathrm{C}\left\{{ }^{1} \mathrm{H}\right\} \mathrm{NMR}\left(\mathrm{CDCl}_{3}, 100 \mathrm{MHz}\right) \delta(\mathrm{ppm})$ 167.3, 145.6, 134.7, 131.6, 129.3, 129.1, 128.4, 128.1, 123.0, 119.0, 114.1, 67.9, 51.7, 28.5; 
HRMS (ESI-TOF) m/z: $[\mathrm{M}+\mathrm{H}]^{+}$Calcd for $\mathrm{C}_{20} \mathrm{H}_{24} \mathrm{BrN}_{2} \mathrm{O}$ 387.1067; Found: 387.1065 .

(Z)-3-Bromo- $N$-(tert-butyl)-4-phenyl-2-(p-tolylamino)but-3-enamide (4e):

White solid, yield: $0.481 \mathrm{~g}(60 \%)$, ethylacetate/petroleum ether $=1: 18, \mathrm{mp} 154-$ $155^{\circ} \mathrm{C} .{ }^{1} \mathrm{H}$ NMR $\left(\mathrm{CDCl}_{3}, 400 \mathrm{MHz}\right) \delta(\mathrm{ppm})$ 7.58-6.59 (m, 10H), 6.49 (s, 1H), 4.70 (s, 1H), $4.48(\mathrm{~s}, 1 \mathrm{H}), 1.38(\mathrm{~s}, 9 \mathrm{H}) ;{ }^{13} \mathrm{C}\left\{{ }^{1} \mathrm{H}\right\} \mathrm{NMR}\left(\mathrm{CDCl}_{3}, 100 \mathrm{MHz}\right) \delta(\mathrm{ppm})$ 167.6, $143.4,134.8,131.8,129.8,129.1,128.5,128.4,128.1,123.1,114.4,68.5,51.6,28.5$, 20.4; HRMS (ESI-TOF) m/z: $[\mathrm{M}+\mathrm{H}]^{+}$Calcd for $\mathrm{C}_{21} \mathrm{H}_{26} \mathrm{BrN}_{2} \mathrm{O}$ 401.1223; Found: 401.1222 .

(Z)-3-Bromo- $N$-(tert-butyl)-2-((4-chlorophenyl)amino)-4-phenylbut-3-enamide (4f):

White solid, yield: $0.690 \mathrm{~g}(82 \%)$, ethylacetate/petroleum ether $=1: 16, \mathrm{mp} 124$ $125{ }^{\circ} \mathrm{C} .{ }^{1} \mathrm{H} \mathrm{NMR}\left(\mathrm{CDCl}_{3}, 400 \mathrm{MHz}\right) \delta(\mathrm{ppm}) 7.59-6.58(\mathrm{~m}, 11 \mathrm{H}), 6.23(\mathrm{~s}, 1 \mathrm{H}), 4.48$ (s, 1H), $1.38(\mathrm{~s}, 9 \mathrm{H}) ;{ }^{13} \mathrm{C}\left\{{ }^{1} \mathrm{H}\right\} \mathrm{NMR}\left(\mathrm{CDCl}_{3}, 100 \mathrm{MHz}\right) \delta(\mathrm{ppm}) 166.9,144.1,134.5$, $131.7,129.2,129.1,128.6,128.2,123.6,122.8,115.1,67.5,51.9,28.5$; HRMS (ESI-TOF) m/z: [M + H] ${ }^{+}$Calcd for $\mathrm{C}_{20} \mathrm{H}_{23} \mathrm{BrClN}_{2} \mathrm{O}$ 421.0677; Found: 421.0677 .

(Z)-N-Benzyl-3-bromo-2-((4-bromophenyl)amino)-4-phenylbut-3-enamide (4g):

White solid, yield: $0.496 \mathrm{~g}(47 \%)$, ethylacetate/petroleum ether $=1: 20, \mathrm{mp} 147-$ $148{ }^{\circ} \mathrm{C} .{ }^{1} \mathrm{H} \mathrm{NMR}\left(\mathrm{CDCl}_{3}, 400 \mathrm{MHz}\right) \delta(\mathrm{ppm})$ 7.59-6.54 (m, 16H), $5.08(\mathrm{~s}, 1 \mathrm{H})$, 4.65-4.49 (m, 3H); ${ }^{13} \mathrm{C}\left\{{ }^{1} \mathrm{H}\right\}$ NMR $\left(\mathrm{CDCl}_{3}, 100 \mathrm{MHz}\right) \delta(\mathrm{ppm})$ 168.0, 144.4, 137.4, $134.3,132.3,132.1,129.1,128.8,128.7,128.2,127.7,127.6,122.0,115.7,111.0$, 67.2, 44.0; HRMS (ESI-TOF) $\mathrm{m} / \mathrm{z}:[\mathrm{M}+\mathrm{Na}]^{+}$Calcd for $\mathrm{C}_{23} \mathrm{H}_{20} \mathrm{Br}^{81} \mathrm{BrN}_{2} \mathrm{NaO}$ 522.9814; Found: 522.9814.

(Z)-3-Bromo- $N$-(tert-butyl)-2-((2-chlorophenyl)amino)-4-phenylbut-3-enamide (4h):

White solid, yield: $0.530 \mathrm{~g}(63 \%)$, ethylacetate/petroleum ether $=1: 20, \mathrm{mp} 118$ $119{ }^{\circ} \mathrm{C} .{ }^{1} \mathrm{H}$ NMR $\left(\mathrm{CDCl}_{3}, 400 \mathrm{MHz}\right) \delta(\mathrm{ppm})$ 7.60-6.58 (m, 10H), 6.26 (s, 1H), 5.72 $(\mathrm{d}, J=4.8 \mathrm{~Hz}, 1 \mathrm{H}), 4.55(\mathrm{~d}, J=5.2 \mathrm{~Hz}, 1 \mathrm{H}), 1.39(\mathrm{~s}, 9 \mathrm{H}) ;{ }^{13} \mathrm{C}\left\{{ }^{1} \mathrm{H}\right\} \mathrm{NMR}\left(\mathrm{CDCl}_{3}\right.$, $100 \mathrm{MHz}) \delta$ (ppm) 166.8, 141.7, 134.6, 131.6, 129.4, 129.1, 128.5, 128.2, 127.8, 122.4, 120.3, 119.0, 112.8, 67.3, 51.9, 28.5; HRMS (ESI-TOF) m/z: $[\mathrm{M}+\mathrm{H}]^{+}$Calcd 
for $\mathrm{C}_{20} \mathrm{H}_{23} \mathrm{BrClN}_{2} \mathrm{O}$ 421.0677; Found: 421.0676.

(Z)-3-Bromo-2-((4-chlorophenyl)amino)- $N$-cyclohexyl-4-phenylbut-3-enamide

(4i):

White solid, yield: $0.688 \mathrm{~g}(77 \%)$, ethylacetate/petroleum ether $=1: 18, \mathrm{mp} 127-$ $128{ }^{\circ} \mathrm{C} .{ }^{1} \mathrm{H} \mathrm{NMR}\left(\mathrm{CDCl}_{3}, 400 \mathrm{MHz}\right) \delta(\mathrm{ppm}) 7.58-6.58(\mathrm{~m}, 10 \mathrm{H}), 6.31(\mathrm{~d}, J=8.0 \mathrm{~Hz}$, $1 \mathrm{H}), 4.90(\mathrm{~s}, 1 \mathrm{H}), 4.55(\mathrm{~s}, 1 \mathrm{H}), 3.88-3.81(\mathrm{~m}, 1 \mathrm{H}), 1.91-1.15(\mathrm{~m}, 10 \mathrm{H}) ;{ }^{13} \mathrm{C}\left\{{ }^{1} \mathrm{H}\right\}$ $\mathrm{NMR}\left(\mathrm{CDCl}_{3}, 100 \mathrm{MHz}\right) \delta(\mathrm{ppm}) 166.8,144.1,131.8,129.2,129.1,128.6,128.2$, 122.4, 115.1, 48.8, 32.8, 32.6, 25.3, 24.6, 24.5; HRMS (ESI-TOF) m/z: $[\mathrm{M}+\mathrm{H}]^{+}$ Calcd for $\mathrm{C}_{22} \mathrm{H}_{25} \mathrm{BrClN}_{2} \mathrm{O}$ 447.0833; Found: 447.0833 .

(Z)-3-Bromo- $N$-(tert-butyl)-2-((3,5-dichlorophenyl)amino)-4-phenylbut-3-enamid e (4j):

White solid, yield: $0.673 \mathrm{~g}(74 \%)$, ethylacetate/petroleum ether $=1: 15, \mathrm{mp} 109$ $110{ }^{\circ} \mathrm{C} .{ }^{1} \mathrm{H} \mathrm{NMR}\left(\mathrm{CDCl}_{3}, 400 \mathrm{MHz}\right) \delta(\mathrm{ppm}) 7.61-6.52(\mathrm{~m}, 9 \mathrm{H}), 6.01(\mathrm{~s}, 1 \mathrm{H}), 5.37(\mathrm{~d}$, $J=4.8 \mathrm{~Hz}, 1 \mathrm{H}), 4.48(\mathrm{~d}, J=4.8 \mathrm{~Hz}, 1 \mathrm{H}), 1.39(\mathrm{~s}, 9 \mathrm{H}) ;{ }^{13} \mathrm{C}\left\{{ }^{1} \mathrm{H}\right\} \mathrm{NMR}\left(\mathrm{CDCl}_{3}, 100\right.$ MHz) $\delta(\mathrm{ppm}) 166.2,147.1,135.5,134.3,131.9,129.1,128.8,128.3,122.6,118.4$, 112.1, 66.4, 52.2, 28.5; HRMS (ESI-TOF) m/z: $[\mathrm{M}+\mathrm{Na}]^{+}$Calcd for $\mathrm{C}_{20} \mathrm{H}_{21} \mathrm{BrCl}_{2} \mathrm{~N}_{2} \mathrm{NaO}$ 477.0107; Found: 477.0106.

(Z)-3-Bromo- $N$-(tert-butyl)-2-((3,4-dichlorophenyl)amino)-4-phenylbut-3-enamid e (4k):

White solid, yield: $0.655 \mathrm{~g}(72 \%)$, ethylacetate/petroleum ether $=1: 20, \mathrm{mp} \mathrm{115-}$ $116{ }^{\circ} \mathrm{C} .{ }^{1} \mathrm{H}$ NMR $\left(\mathrm{CDCl}_{3}, 400 \mathrm{MHz}\right) \delta(\mathrm{ppm}) 7.60-6.49(\mathrm{~m}, 9 \mathrm{H}), 6.06(\mathrm{~s}, 1 \mathrm{H}), 5.25(\mathrm{~d}$, $J=4.8 \mathrm{~Hz}, 1 \mathrm{H}), 4.47(\mathrm{~d}, J=4.8 \mathrm{~Hz}, 1 \mathrm{H}), 1.39(\mathrm{~s}, 9 \mathrm{H}) ;{ }^{13} \mathrm{C}\left\{{ }^{1} \mathrm{H}\right\} \mathrm{NMR}\left(\mathrm{CDCl}_{3}, 100\right.$ MHz) $\delta(\mathrm{ppm}) 166.4,145.0,134.4,133.0,131.9,130.8,129.1,128.7,128.3,122.6$, 121.4, 115.1, 113.5, 66.8, 52.1, 28.5; HRMS (ESI-TOF) m/z: $[\mathrm{M}+\mathrm{Na}]^{+}$Calcd for $\mathrm{C}_{20} \mathrm{H}_{21} \mathrm{BrCl}_{2} \mathrm{~N}_{2} \mathrm{NaO}$ 477.0107; Found: 477.0106.

(Z)-3-Bromo- $N$-cyclohexyl-2-((4-fluorophenyl)amino)-4-phenylbut-3-enamide

(4l):

White solid, yield: $0.663 \mathrm{~g}(77 \%)$, ethylacetate/petroleum ether $=1: 16, \mathrm{mp} 118$ $119{ }^{\circ} \mathrm{C} .{ }^{1} \mathrm{H}$ NMR $\left(\mathrm{CDCl}_{3}, 400 \mathrm{MHz}\right) \delta(\mathrm{ppm})$ 7.58-6.39 (m, 11H), $4.53(\mathrm{~s}, 1 \mathrm{H})$, 3.88-3.81 (m, 1H), 1.92-0.86 (m, 10H); ${ }^{13} \mathrm{C}\left\{{ }^{1} \mathrm{H}\right\}$ NMR $\left(\mathrm{CDCl}_{3}, 100 \mathrm{MHz}\right) \delta(\mathrm{ppm})$ 
$167.1,156.7\left(\mathrm{~d}, J_{\mathrm{C}-\mathrm{F}}=235.8 \mathrm{~Hz}\right), 141.8\left(\mathrm{~d}, J_{\mathrm{C}-\mathrm{F}}=1.9 \mathrm{~Hz}\right), 134.6,131.9,129.1,128.6$, 128.2, 122.6, $115.8\left(\mathrm{~d}, J_{\mathrm{C}-\mathrm{F}}=22.3 \mathrm{~Hz}\right), 115.1\left(\mathrm{~d}, J_{\mathrm{C}-\mathrm{F}}=7.5 \mathrm{~Hz}\right), 68.1,48.7,32.9,32.7$, 25.4, 24.7, 24.6; HRMS (ESI-TOF) m/z: $[\mathrm{M}+\mathrm{H}]^{+}$Calcd for $\mathrm{C}_{22} \mathrm{H}_{25} \mathrm{BrFN}_{2} \mathrm{O} 431.1129$; Found: 431.1128 .

\section{(Z)-3-Bromo- $N$-cyclohexyl-4-phenyl-2-(phenylamino)but-3-enamide (4m):}

White solid, yield: $0.570 \mathrm{~g}(69 \%)$, ethylacetate/petroleum ether $=1: 20, \mathrm{mp} 130-$ $131{ }^{\circ} \mathrm{C} .{ }^{1} \mathrm{H} \mathrm{NMR}\left(\mathrm{CDCl}_{3}, 400 \mathrm{MHz}\right) \delta(\mathrm{ppm})$ 7.59-6.42 (m, 12H), $4.90(\mathrm{~d}, J=4.4 \mathrm{~Hz}$, $1 \mathrm{H}), 4.58(\mathrm{~d}, J=4.8 \mathrm{~Hz}, 1 \mathrm{H}), 3.89-3.82(\mathrm{~m}, 1 \mathrm{H}), 1.95-1.15(\mathrm{~m}, 10 \mathrm{H}) ;{ }^{13} \mathrm{C}\left\{{ }^{1} \mathrm{H}\right\} \mathrm{NMR}$ $\left(\mathrm{CDCl}_{3}, 100 \mathrm{MHz}\right) \delta(\mathrm{ppm}) 167.3,145.6,134.7,131.8,129.4,129.1,128.5,128.1$, 122.7, 119.1, 114.1, 67.6, 48.7, 32.9, 32.7, 25.4, 24.7, 24.6; HRMS (ESI-TOF) m/z: $[\mathrm{M}+\mathrm{H}]^{+}$Calcd for $\mathrm{C}_{22} \mathrm{H}_{26} \mathrm{BrN}_{2} \mathrm{O}$ 413.1223; Found: 413.1223.

(Z)- $N$-((3s,5s,7s)-Adamantan-1-yl)-3-bromo-2-((4-chlorophenyl)amino)-4-phenyl but-3-enamide (4n):

White solid, yield: $0.838 \mathrm{~g}(84 \%)$, ethylacetate/petroleum ether $=1: 15, \mathrm{mp} 207-$ $208{ }^{\circ} \mathrm{C} .{ }^{1} \mathrm{H} \mathrm{NMR}\left(\mathrm{CDCl}_{3}, 400 \mathrm{MHz}\right) \delta(\mathrm{ppm})$ 7.58-6.57 (m, 10H), 6.09 (s, 1H), 5.05 $(\mathrm{d}, J=4.4 \mathrm{~Hz}, 1 \mathrm{H}), 4.47(\mathrm{~d}, J=4.4 \mathrm{~Hz}, 1 \mathrm{H}), 2.08-1.68(\mathrm{~m}, 15 \mathrm{H}),{ }^{13} \mathrm{C}\left\{{ }^{1} \mathrm{H}\right\} \mathrm{NMR}$ $\left(\mathrm{CDCl}_{3}, 100 \mathrm{MHz}\right) \delta(\mathrm{ppm}) 166.5,144.1,134.5,131.6,129.2,129.1,128.5,128.2$, 123.5, 123.0, 115.1, 67.5, 52.5, 41.3, 36.2, 29.3; HRMS (ESI-TOF) m/z: $[\mathrm{M}+\mathrm{Na}]^{+}$ Calcd for $\mathrm{C}_{26} \mathrm{H}_{28} \mathrm{BrClN}_{2} \mathrm{NaO}$ 521.0966; Found: 521.0965.

(Z)- $N$-((3s,5s,7s)-Adamantan-1-yl)-3-bromo-4-phenyl-2-(phenylamino)but-3-ena mide (40):

White solid, yield: 0.698g (75\%), ethylacetate/petroleum ether $=1: 16, \mathrm{mp} 166-$ $167{ }^{\circ} \mathrm{C} .{ }^{1} \mathrm{H} \mathrm{NMR}\left(\mathrm{CDCl}_{3}, 400 \mathrm{MHz}\right) \delta(\mathrm{ppm})$ 7.59-6.24 (m, 12H), $4.87(\mathrm{~d}, J=7.2 \mathrm{~Hz}$, $1 \mathrm{H}), 4.51(\mathrm{~s}, 1 \mathrm{H}), 2.08-1.68(\mathrm{~m}, 15 \mathrm{H}) ;{ }^{13} \mathrm{C}\left\{{ }^{1} \mathrm{H}\right\} \mathrm{NMR}\left(\mathrm{CDCl}_{3}, 100 \mathrm{MHz}\right) \delta(\mathrm{ppm})$ 167.0, 145.7, 134.7, 131.6, 129.3, 129.1, 128.4, 128.1, 123.2, 119.0, 114.1, 67.9, 52.4, 41.3, 36.2, 29.3; HRMS (ESI-TOF) $\mathrm{m} / \mathrm{z}:[\mathrm{M}+\mathrm{Na}]^{+}$Calcd for $\mathrm{C}_{26} \mathrm{H}_{29} \mathrm{BrN}_{2} \mathrm{NaO}$ 487.1355; Found: 487.1355.

(Z)- $N$-((3s,5s,7s)-Adamantan-1-yl)-3-bromo-2-((4-bromophenyl)amino)-4-phenyl but-3-enamide (4p):

White solid, yield: $0.836 \mathrm{~g}(77 \%)$, ethylacetate/petroleum ether $=1: 20, \mathrm{mp} 167-$ 
$168{ }^{\circ} \mathrm{C} .{ }^{1} \mathrm{H}$ NMR $\left(\mathrm{CDCl}_{3}, 400 \mathrm{MHz}\right) \delta(\mathrm{ppm})$ 7.58-6.52 (m, 10H), $6.06(\mathrm{~s}, 1 \mathrm{H}), 5.08$ $(\mathrm{d}, J=4.4 \mathrm{~Hz}, 1 \mathrm{H}), 4.46(\mathrm{~d}, J=4.8 \mathrm{~Hz}, 1 \mathrm{H}), 2.08-1.68(\mathrm{~m}, 15 \mathrm{H}),{ }^{13} \mathrm{C}\left\{{ }^{1} \mathrm{H}\right\} \mathrm{NMR}$ $\left(\mathrm{CDCl}_{3}, 100 \mathrm{MHz}\right) \delta(\mathrm{ppm}) 166.4,144.6,134.5,132.0,131.6,129.1,128.6,128.2$, 123.0, 115.5, 110.6, 67.3, 52.6, 41.3, 36.2, 29.3; HRMS (ESI-TOF) m/z: $[\mathrm{M}+\mathrm{Na}]^{+}$ Calcd for $\mathrm{C}_{26} \mathrm{H}_{28} \mathrm{Br}^{81} \mathrm{BrN}_{2} \mathrm{NaO}$ 567.0440; Found: 567.0439.

(Z)- $N$-((3s,5s,7s)-Adamantan-1-yl)-3-bromo-2-((3-chlorophenyl)amino)-4-phenyl but-3-enamide (4q):

White solid, yield: $0.739 \mathrm{~g}(74 \%)$, ethylacetate/petroleum ether $=1: 18, \mathrm{mp} 135$ $136{ }^{\circ} \mathrm{C} .{ }^{1} \mathrm{H}$ NMR $\left(\mathrm{CDCl}_{3}, 400 \mathrm{MHz}\right) \delta(\mathrm{ppm})$ 7.60-6.52 (m, 10H), $6.02(\mathrm{~s}, 1 \mathrm{H}), 5.16$ $(\mathrm{d}, J=4.8 \mathrm{~Hz}, 1 \mathrm{H}), 4.49(\mathrm{~d}, J=4.8 \mathrm{~Hz}, 1 \mathrm{H}), 2.09-1.68(\mathrm{~m}, 15 \mathrm{H}) ;{ }^{13} \mathrm{C}\left\{{ }^{1} \mathrm{H}\right\} \mathrm{NMR}$ $\left(\mathrm{CDCl}_{3}, 100 \mathrm{MHz}\right) \delta$ (ppm) 166.3, 146.7, 135.0, 134.5, 131.7, 130.3, 129.1, 128.6, 128.2, 123.0, 118.6, 113.8, 112.1, 67.0, 52.6, 41.3, 36.2, 29.3; HRMS (ESI-TOF) m/z: $[\mathrm{M}+\mathrm{Na}]^{+}$Calcd for $\mathrm{C}_{26} \mathrm{H}_{28} \mathrm{BrClN}{ }_{2} \mathrm{NaO}$ 521.0966; Found: 521.0965.

(Z)- $N$-((3s,5s,7s)-Adamantan-1-yl)-3-bromo-4-(4-chlorophenyl)-2-((4-chlorophen yl)amino)but-3-enamide (4r):

White solid, yield: $0.757 \mathrm{~g}(71 \%)$, ethylacetate/petroleum ether $=1: 15, \mathrm{mp} 167-$ $168{ }^{\circ} \mathrm{C} .{ }^{1} \mathrm{H} \mathrm{NMR}\left(\mathrm{CDCl}_{3}, 400 \mathrm{MHz}\right) \delta(\mathrm{ppm}) 7.51-6.56(\mathrm{~m}, 9 \mathrm{H}), 6.10(\mathrm{~s}, 1 \mathrm{H}), 5.02(\mathrm{~d}$, $J=4.8 \mathrm{~Hz}, 1 \mathrm{H}), 4.45(\mathrm{~d}, J=4.8 \mathrm{~Hz}, 1 \mathrm{H}), 2.08-1.68(\mathrm{~m}, 15 \mathrm{H}) ;{ }^{13} \mathrm{C}\left\{{ }^{1} \mathrm{H}\right\} \mathrm{NMR}\left(\mathrm{CDCl}_{3}\right.$, $100 \mathrm{MHz}) \delta(\mathrm{ppm}) 166.3,144.0,134.3,133.0,130.4,130.3,129.2,128.4,123.6$, 123.5, 115.1, 67.5, 52.6, 41.3, 36.1, 29.3; HRMS (ESI-TOF) m/z: $[\mathrm{M}+\mathrm{Na}]^{+}$Calcd for $\mathrm{C}_{26} \mathrm{H}_{27}{ }^{81} \mathrm{BrCl}_{2} \mathrm{~N}_{2} \mathrm{NaO}$ 557.0556; Found: 557.0552.

(Z)- $N$-((3s,5s,7s)-Adamantan-1-yl)-3-bromo-4-(4-chlorophenyl)-2-(phenylamino) but-3-enamide (4s):

White solid, yield: $0.639 \mathrm{~g}(64 \%)$, ethylacetate/petroleum ether $=1: 20, \mathrm{mp} 140$ $141{ }^{\circ} \mathrm{C} .{ }^{1} \mathrm{H} \mathrm{NMR}\left(\mathrm{CDCl}_{3}, 400 \mathrm{MHz}\right) \delta(\mathrm{ppm}) 7.52-6.40(\mathrm{~m}, 11 \mathrm{H}), 4.87(\mathrm{~d}, J=4.4 \mathrm{~Hz}$, $1 \mathrm{H}), 4.51(\mathrm{~d}, J=4.8 \mathrm{~Hz}, 1 \mathrm{H}), 1.38(\mathrm{~s}, 9 \mathrm{H}) ;{ }^{13} \mathrm{C}\left\{{ }^{1} \mathrm{H}\right\} \mathrm{NMR}\left(\mathrm{CDCl}_{3}, 100 \mathrm{MHz}\right) \delta(\mathrm{ppm})$ 167.2, 145.5, 134.2, 133.1, 130.4, 130.3, 129.4, 128.3, 123.6, 119.2, 114.1, 67.9, 51.8, 28.5; HRMS (ESI-TOF) m/z: $[\mathrm{M}+\mathrm{H}]^{+}$Calcd for $\mathrm{C}_{26} \mathrm{H}_{29} \mathrm{BrClN}_{2} \mathrm{O}$ 499.1146; Found: 499.1145. 
mide (4t):

White solid, yield: $0.556 \mathrm{~g}(58 \%)$, ethylacetate/petroleum ether $=1: 16, \mathrm{mp} 195$ $196{ }^{\circ} \mathrm{C} .{ }^{1} \mathrm{H}$ NMR $\left(\mathrm{CDCl}_{3}, 400 \mathrm{MHz}\right) \delta(\mathrm{ppm})$ 7.58-6.35 (m, 11H), 4.69 (s, 1H), 4.47 $(\mathrm{s}, 1 \mathrm{H}), 2.25(\mathrm{~s}, 3 \mathrm{H}), 2.08-1.68(\mathrm{~m}, 15 \mathrm{H}) ;{ }^{13} \mathrm{C}\left\{{ }^{1} \mathrm{H}\right\} \mathrm{NMR}\left(\mathrm{CDCl}_{3}, 100 \mathrm{MHz}\right) \delta(\mathrm{ppm})$ $167.3,143.5,131.8,130.0,129.2,128.5,128.4,128.2,114.4,68.5,52.3,41.4,36.3$, 29.4, 20.5; HRMS (ESI-TOF) m/z: $[\mathrm{M}+\mathrm{H}]^{+}$Calcd for $\mathrm{C}_{27} \mathrm{H}_{32} \mathrm{BrN}_{2} \mathrm{O}$ 479.1693; Found: 479.1692.

(Z)- $N$-((3s,5s,7s)-Adamantan-1-yl)-3-bromo-2-((4-fluorophenyl)amino)-4-phenylb ut-3-enamide (4u):

White solid, yield: $0.696 \mathrm{~g}(72 \%)$, ethylacetate/petroleum ether $=1: 15, \mathrm{mp} 150$ $151{ }^{\circ} \mathrm{C} .{ }^{1} \mathrm{H} \mathrm{NMR}\left(\mathrm{CDCl}_{3}, 400 \mathrm{MHz}\right) \delta(\mathrm{ppm}) 7.58-6.21(\mathrm{~m}, 11 \mathrm{H}), 4.85(\mathrm{~d}, J=4.4 \mathrm{~Hz}$, $1 \mathrm{H}), 4.44(\mathrm{~d}, J=4.8 \mathrm{~Hz}, 1 \mathrm{H}), 2.08-1.68(\mathrm{~m}, 15 \mathrm{H}) ;{ }^{13} \mathrm{C}\left\{{ }^{1} \mathrm{H}\right\} \mathrm{NMR}\left(\mathrm{CDCl}_{3}, 100 \mathrm{MHz}\right)$ $\delta(\mathrm{ppm}) 166.8,156.6\left(\mathrm{~d}, J_{\mathrm{C}-\mathrm{F}}=235.4 \mathrm{~Hz}\right), 142.0,134.6,131.7,129.1,128.5,128.2$, $123.2,115.7\left(\mathrm{~d}, J_{\mathrm{C}-\mathrm{F}}=22.3 \mathrm{~Hz}\right), 115.1\left(\mathrm{~d}, J_{\mathrm{C}-\mathrm{F}}=7.5 \mathrm{~Hz}\right), 68.3,52.4,41.3,36.2,29.3$; HRMS (ESI-TOF) m/z: $[\mathrm{M}+\mathrm{H}]^{+}$Calcd for $\mathrm{C}_{26} \mathrm{H}_{29} \mathrm{BrFN}_{2} \mathrm{O}$ 483.1442; Found: 483.1440 .

\section{(Z)-3-Bromo-4-(4-chlorophenyl)-2-((4-chlorophenyl)amino)- $N$-cyclohexylbut-3-e} namide (4v):

White solid, yield: $0.683 \mathrm{~g}(71 \%)$, ethylacetate/petroleum ether $=1: 20, \mathrm{mp} 140$ $142{ }^{\circ} \mathrm{C} .{ }^{1} \mathrm{H}$ NMR $\left(\mathrm{CDCl}_{3}, 400 \mathrm{MHz}\right) \delta(\mathrm{ppm}) 7.51-6.57(\mathrm{~m}, 9 \mathrm{H}), 6.30(\mathrm{~d}, J=6.4 \mathrm{~Hz}$, $1 \mathrm{H}), 5.01(\mathrm{~d}, J=3.2 \mathrm{~Hz}, 1 \mathrm{H}), 4.53(\mathrm{~d}, J=2.8 \mathrm{~Hz}, 1 \mathrm{H}), 3.87-3.81(\mathrm{~m}, 1 \mathrm{H}), 1.95-1.14$ $(\mathrm{m}, 10 \mathrm{H}) ;{ }^{13} \mathrm{C}\left\{{ }^{1} \mathrm{H}\right\} \mathrm{NMR}\left(\mathrm{CDCl}_{3}, 100 \mathrm{MHz}\right) \delta(\mathrm{ppm})$ 166.7, 143.9, 130.5, 130.4, 129.2, 128.4, 123.8, 123.0, 115.1, 67.3, 48.8, 32.8, 32.7, 25.4, 24.6, 24.5; HRMS (ESI-TOF) m/z: $[\mathrm{M}+\mathrm{Na}]^{+}$Calcd for $\mathrm{C}_{22} \mathrm{H}_{23} \mathrm{BrCl}_{2} \mathrm{~N}_{2} \mathrm{NaO}$ 503.0263; Found: 503.0263.

\section{(Z)-3-Bromo-4-(4-chlorophenyl)- $N$-cyclohexyl-2-(m-tolylamino)but-3-enamide} (4w):

White solid, yield: $0.618 \mathrm{~g}(67 \%)$, ethylacetate/petroleum ether $=1: 20, \mathrm{mp} 111-$ $112{ }^{\circ} \mathrm{C} .{ }^{1} \mathrm{H} \mathrm{NMR}\left(\mathrm{CDCl}_{3}, 400 \mathrm{MHz}\right) \delta(\mathrm{ppm})$ 7.52-6.46 (m, 10H), 4.75-4.56 (m, 2H), 3.82-3.80 (m, 1H), $2.28(\mathrm{~s}, 3 \mathrm{H}), 2.27-1.18(\mathrm{~m}, 10 \mathrm{H}) ;{ }^{13} \mathrm{C}\left\{{ }^{1} \mathrm{H}\right\}$ NMR $\left(\mathrm{CDCl}_{3}, 100\right.$ 
MHz) $\delta(\mathrm{ppm}) 167.3,145.6,139.3,134.2,133.2,130.6,130.4,129.3,128.4,123.2$, 120.3, 115.0, 111.1, 67.9, 48.7, 32.7, 25.4, 24.6, 21.6; HRMS (ESI-TOF) m/z: [M + $\mathrm{Na}]^{+}$Calcd for $\mathrm{C}_{23} \mathrm{H}_{26}{ }^{81} \mathrm{BrClN}_{2} \mathrm{NaO}$ 485.0789; Found: 485.0789 .

(Z)- $N$-((3s,5s,7s)-Adamantan-1-yl)-3-bromo-2-((4-chlorophenyl)amino)-4-(4-fluor ophenyl)but-3-enamide (4x):

White solid, yield: $0.724 \mathrm{~g}(70 \%)$, ethylacetate/petroleum ether $=1: 18, \mathrm{mp} 186-$ $187{ }^{\circ} \mathrm{C} .{ }^{1} \mathrm{H} \mathrm{NMR}\left(\mathrm{CDCl}_{3}, 400 \mathrm{MHz}\right) \delta(\mathrm{ppm})$ 7.58-6.57 (m, 9H), 6.09 (s, 1H), $5.01(\mathrm{~d}$, $J=4.8 \mathrm{~Hz}, 1 \mathrm{H}), 4.45(\mathrm{~d}, J=4.8 \mathrm{~Hz}, 1 \mathrm{H}), 2.09-1.68(\mathrm{~m}, 15 \mathrm{H}) ;{ }^{13} \mathrm{C}\left\{{ }^{1} \mathrm{H}\right\} \mathrm{NMR}\left(\mathrm{CDCl}_{3}\right.$, $100 \mathrm{MHz}) \delta(\mathrm{ppm}) 166.5,162.5\left(\mathrm{~d}, J_{\mathrm{C}-\mathrm{F}}=247.6 \mathrm{~Hz}\right), 144.1,131.0\left(\mathrm{~d}, J_{\mathrm{C}-\mathrm{F}}=8.1 \mathrm{~Hz}\right)$, $130.6\left(\mathrm{~d}, J_{\mathrm{C}-\mathrm{F}}=3.3 \mathrm{~Hz}\right), 130.5,129.3,123.7,122.8\left(\mathrm{~d}, J_{\mathrm{C}-\mathrm{F}}=1.7 \mathrm{~Hz}\right), 115.3\left(\mathrm{~d}, J_{\mathrm{C}-\mathrm{F}}=\right.$ $21.5 \mathrm{~Hz}), 115.1,67.6,52.6,41.4,36.2,29.4$; HRMS (ESI-TOF) m/z: $[\mathrm{M}+\mathrm{H}]^{+}$Calcd for $\mathrm{C}_{26} \mathrm{H}_{28} \mathrm{BrClFN}_{2} \mathrm{O}$ 517.1052; Found: 517.1051.

(Z)-3-Bromo- $N$-butyl-2-((4-chlorophenyl)amino)-4-phenylbut-3-enamide (4y):

White solid, yield: 0.648g (77\%), ethylacetate/petroleum ether $=1: 20, \mathrm{mp} 82-84{ }^{\circ} \mathrm{C}$. ${ }^{1} \mathrm{H} \mathrm{NMR}\left(\mathrm{CDCl}_{3}, 400 \mathrm{MHz}\right) \delta(\mathrm{ppm})$ 7.59-6.44 (m, 11H), $4.58(\mathrm{~s}, 1 \mathrm{H}), 3.36-3.30(\mathrm{~m}$, 1H), 1.53-0.89 (m, 7H); ${ }^{13} \mathrm{C}\left\{{ }^{1} \mathrm{H}\right\} \mathrm{NMR}\left(\mathrm{CDCl}_{3}, 100 \mathrm{MHz}\right) \delta(\mathrm{ppm})$ 167.9, 144.1, $134.5,132.0,129.3,129.2,128.7,128.3,123.8,122.4,115.2,67.3,39.8,31.5,20.0$, 13.7; HRMS (ESI-TOF) m/z: $[\mathrm{M}+\mathrm{H}]^{+}$Calcd for $\mathrm{C}_{20} \mathrm{H}_{23} \mathrm{BrClN}_{2} \mathrm{O}$ 421.0677; Found: 421.0676 .

(Z)-3-Bromo- $N$-butyl-4-phenyl-2-((4-(trifluoromethyl)phenyl)amino)but-3-enami de (4z):

Light yellow oil, yield: 0.655g (72\%), ethylacetate/petroleum ether $=1: 18 .{ }^{1} \mathrm{H}$ NMR $\left(\mathrm{CDCl}_{3}, 400 \mathrm{MHz}\right) \delta(\mathrm{ppm}) 7.59-6.40(\mathrm{~m}, 11 \mathrm{H}), 6.26(\mathrm{~s}, 1 \mathrm{H}), 5.55-5.36(\mathrm{~m}, 1 \mathrm{H}), 4.67$ $(\mathrm{d}, J=3.6 \mathrm{~Hz}, 1 \mathrm{H}), 3.39-0.89(\mathrm{~m}, 9 \mathrm{H}) ;{ }^{13} \mathrm{C}\left\{{ }^{1} \mathrm{H}\right\} \mathrm{NMR}\left(\mathrm{CDCl}_{3}, 100 \mathrm{MHz}\right) \delta(\mathrm{ppm})$ $167.5,146.8\left(\mathrm{~d}, J_{\mathrm{C}-\mathrm{F}}=226.1 \mathrm{~Hz}\right), 134.3,132.2,131.9,129.8,129.1,129.0,128.7$, $128.2,126.6\left(\mathrm{q}, J_{\mathrm{C}-\mathrm{F}}=3.0 \mathrm{~Hz}\right), 122.3,122.2,116.9,113.1,110.2,66.2,39.9,31.4$, 19.9, 13.6; HRMS (ESI-TOF) m/z: $[\mathrm{M}+\mathrm{H}]^{+}$Calcd for $\mathrm{C}_{21} \mathrm{H}_{23} \mathrm{BrF}_{3} \mathrm{~N}_{2} \mathrm{O}$ 455.0940; Found: 455.0940.

$N^{3}$-((3s, $\left.5 s, 7 s\right)$-Adamantan-1-yl)-5-(((3s,5s,7s)-adamantan-1-yl)amino)-1-(4-bromo phenyl)- $N^{2}$-(tert-butyl)-4-phenyl-1H-pyrrole-2,3-dicarboxamide (6a): 
White solid, yield: $0.528 \mathrm{~g}(73 \%)$, ethylacetate/petroleum ether $=1: 15, \mathrm{mp} \mathrm{278-}$ $279{ }^{\circ} \mathrm{C} .{ }^{1} \mathrm{H}$ NMR $\left(\mathrm{CDCl}_{3}, 400 \mathrm{MHz}\right) \delta(\mathrm{ppm}) 8.59$ (s, 1H), 7.56-7.15 (m, 9H), 4.88 (s, 1H), $2.93(\mathrm{~s}, 1 \mathrm{H}), 1.96-0.93(\mathrm{~m}, 39 \mathrm{H}) ;{ }^{13} \mathrm{C}\left\{{ }^{1} \mathrm{H}\right\} \mathrm{NMR}\left(\mathrm{CDCl}_{3}, 100 \mathrm{MHz}\right) \delta(\mathrm{ppm})$ $166.3,160.4,137.8,134.9,134.3,131.7,130.4,129.2$, 128.5, 126.9, 124.2, 121.8, 121.3, 116.9, 55.4, 52.1, 51.2, 43.5, 42.5, 40.8, 36.2, 35.9, 29.5, 28.7; HRMS (ESI-TOF) m/z: $[\mathrm{M}+\mathrm{H}]^{+}$Calcd for $\mathrm{C}_{42} \mathrm{H}_{52} \mathrm{BrN}_{4} \mathrm{O}_{2}$ 723.3268; Found: 723.3266 .

$N^{3}$-((3s,5s,7s)-Adamantan-1-yl)-5-(((3s,5s,7s)-adamantan-1-yl)amino)- $N^{2}$-(tert-but yl)-1,4-diphenyl-1H-pyrrole-2,3-dicarboxamide (6b):

White solid, yield: $0.432 \mathrm{~g}(67 \%)$, ethylacetate/petroleum ether $=1: 12, \mathrm{mp} 279$ $280{ }^{\circ} \mathrm{C} .{ }^{1} \mathrm{H} \mathrm{NMR}\left(\mathrm{CDCl}_{3}, 400 \mathrm{MHz}\right) \delta(\mathrm{ppm}) 8.47$ (s, 1H), 7.46-7.26 (m, 10H), 4.95 $(\mathrm{s}, 1 \mathrm{H}), 3.04(\mathrm{~s}, 1 \mathrm{H}), 1.97-0.91(\mathrm{~m}, 39 \mathrm{H}) ;{ }^{13} \mathrm{C}\left\{{ }^{1} \mathrm{H}\right\} \mathrm{NMR}\left(\mathrm{CDCl}_{3}, 100 \mathrm{MHz}\right) \delta(\mathrm{ppm})$ $166.5,160.6,138.6,135.2,134.5,130.5,128.7,128.4$, 127.5, 126.6, 124.2, 121.5, 116.1, 55.2, 52.0, 51.1, 43.4, 42.5, 40.8, 36.2, 35.9, 29.6, 28.7; HRMS (ESI-TOF) m/z: $[\mathrm{M}+\mathrm{H}]^{+}$Calcd for $\mathrm{C}_{42} \mathrm{H}_{53} \mathrm{~N}_{4} \mathrm{O}_{2}$ 645.4163; Found: 645.4160.

$N^{3}-\left((3 s, 5 s, 7 s)\right.$-Adamantan-1-yl)-5-(((3s,5s,7s)-adamantan-1-yl)amino)- $N^{2}$-(tert-but yl)-1-(4-chlorophenyl)-4-phenyl-1H-pyrrole-2,3-dicarboxamide (6c):

White solid, yield: $0.523 \mathrm{~g}(77 \%)$, ethylacetate/petroleum ether $=1: 15, \mathrm{mp} 240$ $241{ }^{\circ} \mathrm{C} .{ }^{1} \mathrm{H} \mathrm{NMR}\left(\mathrm{CDCl}_{3}, 400 \mathrm{MHz}\right) \delta(\mathrm{ppm}) 8.59$ (s, 1H), 7.41-7.21 (m, 9H), 4.88 (s, 1H), $2.93(\mathrm{~s}, 1 \mathrm{H}), 1.97-0.93(\mathrm{~m}, 39 \mathrm{H}) ;{ }^{13} \mathrm{C}\left\{{ }^{1} \mathrm{H}\right\} \mathrm{NMR}\left(\mathrm{CDCl}_{3}, 100 \mathrm{MHz}\right) \delta(\mathrm{ppm})$ $166.4,160.4,137.3,134.9,134.3,133.2,130.4,128.8,128.7,128.5,128.9,124.3$, $121.7,116.9,55.4,52.1,51.2,43.5,42.5,40.8,36.2,35.9,29.5,28.7$; HRMS (ESI-TOF) m/z: $[\mathrm{M}+\mathrm{Na}]^{+}$Calcd for $\mathrm{C}_{42} \mathrm{H}_{51} \mathrm{ClN}_{4} \mathrm{NaO}_{2}$ 701.3593; Found: 701.3590.

$N^{2}, N^{3}$-Di-tert-butyl-5-(tert-butylamino)-1-(3-chlorophenyl)-4-phenyl-1H-pyrrole-

\section{2,3-dicarboxamide (6d):}

White solid, yield: $0.377 \mathrm{~g}(72 \%)$, ethylacetate/petroleum ether $=1: 15, \mathrm{mp} \mathrm{251-}$ $252{ }^{\circ} \mathrm{C} .{ }^{1} \mathrm{H}$ NMR $\left(\mathrm{CDCl}_{3}, 400 \mathrm{MHz}\right) \delta(\mathrm{ppm}) 8.74(\mathrm{~s}, 1 \mathrm{H}), 7.51-7.27$ (m, 9H), 5.07 (s, $1 \mathrm{H}), 2.86(\mathrm{~s}, 1 \mathrm{H}), 1.71-0.52(\mathrm{~m}, 27 \mathrm{H}) ;{ }^{13} \mathrm{C}\left\{{ }^{1} \mathrm{H}\right\} \mathrm{NMR}\left(\mathrm{CDCl}_{3}, 100 \mathrm{MHz}\right) \delta(\mathrm{ppm})$ 166.6, 160.2, 136.7, 135.0, 134.9, 132.2, 130.5, 129.9, 129.8, 128.9, 128.4, 126.9, 126.8, 124.9, 120.8, 116.7, 54.5, 51.4, 50.9, 30.2, 28.6, 27.9; HRMS (ESI-TOF) m/z: $[\mathrm{M}+\mathrm{H}]^{+}$Calcd for $\mathrm{C}_{30} \mathrm{H}_{40} \mathrm{ClN}_{4} \mathrm{O}_{2}$ 523.2834; Found: 523.2836. 
$N^{2}, N^{3}$-Di-tert-butyl-5-(tert-butylamino)-4-phenyl-1-(4-(trifluoromethyl)phenyl)-1 $H$-pyrrole-2,3-dicarboxamide (6e):

White solid, yield: $0.429 \mathrm{~g}(77 \%)$, ethylacetate/petroleum ether $=1: 12, \mathrm{mp} \mathrm{243-}$ $245^{\circ} \mathrm{C} .{ }^{1} \mathrm{H} \mathrm{NMR}\left(\mathrm{CDCl}_{3}, 400 \mathrm{MHz}\right) \delta(\mathrm{ppm}) 8.69(\mathrm{~s}, 1 \mathrm{H}), 7.71-7.27$ (m, 9H), $5.04(\mathrm{~s}$, $1 \mathrm{H}), 2.96(\mathrm{~s}, 1 \mathrm{H}), 1.34-0.46(\mathrm{~m}, 27 \mathrm{H}) ;{ }^{13} \mathrm{C}\left\{{ }^{1} \mathrm{H}\right\} \mathrm{NMR}\left(\mathrm{CDCl}_{3}, 100 \mathrm{MHz}\right) \delta(\mathrm{ppm})$ $166.4,160.2,141.8,135.1,134.6,130.2,129.3\left(\mathrm{~d}, J_{\mathrm{C}-\mathrm{F}}=3.4 \mathrm{~Hz}\right), 128.6,128.0,127.1$, $125.6\left(\mathrm{q}, J_{\mathrm{C}-\mathrm{F}}=3.6 \mathrm{~Hz}\right), 124.7,124.0\left(\mathrm{~d}, J_{\mathrm{C}-\mathrm{F}}=270.4 \mathrm{~Hz}\right), 121.9,117.5,55.2,51.5$, 51.2, 30.1, 28.6, 27.9; HRMS (ESI-TOF) m/z: $[\mathrm{M}+\mathrm{H}]^{+}$Calcd for $\mathrm{C}_{31} \mathrm{H}_{40} \mathrm{~F}_{3} \mathrm{~N}_{4} \mathrm{O}_{2}$ 557.3098; Found: 557.3097.

$N^{2}, N^{3}$-Di-tert-butyl-5-(tert-butylamino)-1,4-diphenyl-1H-pyrrole-2,3-dicarboxami de (6f):

White solid, yield: $0.342 \mathrm{~g}(70 \%)$, ethylacetate/petroleum ether $=1: 12, \mathrm{mp} 242-$ $243{ }^{\circ} \mathrm{C} .{ }^{1} \mathrm{H}$ NMR $\left(\mathrm{CDCl}_{3}, 400 \mathrm{MHz}\right) \delta(\mathrm{ppm}) 8.43$ (s, 1H), 7.37-7.18 (m, 10H), 5.00 $(\mathrm{s}, 1 \mathrm{H}), 3.01(\mathrm{~s}, 1 \mathrm{H}), 1.25-0.40(\mathrm{~m}, 27 \mathrm{H}) ;{ }^{13} \mathrm{C}\left\{{ }^{1} \mathrm{H}\right\} \mathrm{NMR}\left(\mathrm{CDCl}_{3}, 100 \mathrm{MHz}\right) \delta(\mathrm{ppm})$ $166.7,160.5,138.5,135.3,135.1,130.3,128.7,128.4,127.6,127.4,126.7,124.4$, 116.0, 55.0, 51.3, 51.1, 30.1, 28.6, 28.0; HRMS (ESI-TOF) m/z: $[\mathrm{M}+\mathrm{H}]^{+}$Calcd for $\mathrm{C}_{30} \mathrm{H}_{41} \mathrm{~N}_{4} \mathrm{O}_{2}$ 489.3224; Found: 489.3224.

$N^{2}, N^{3}$-Di-tert-butyl-5-(tert-butylamino)-4-phenyl-1-(p-tolyl)-1H-pyrrole-2,3-dicar boxamide (6g):

White solid, yield: $0.292 \mathrm{~g}(58 \%)$, ethylacetate/petroleum ether $=1: 14, \mathrm{mp} 237-$ $239{ }^{\circ} \mathrm{C} .{ }^{1} \mathrm{H} \mathrm{NMR}\left(\mathrm{CDCl}_{3}, 400 \mathrm{MHz}\right) \delta(\mathrm{ppm}) 8.49$ (s, 1H), 7.38-7.12 (m, 9H), 5.07 (s, $1 \mathrm{H}), 3.12(\mathrm{~s}, 1 \mathrm{H}), 2.40(\mathrm{~s}, 3 \mathrm{H}), 1.33-0.49(\mathrm{~m}, 27 \mathrm{H}) ;{ }^{13} \mathrm{C}\left\{{ }^{1} \mathrm{H}\right\} \mathrm{NMR}\left(\mathrm{CDCl}_{3}, 100 \mathrm{MHz}\right)$ $\delta(\mathrm{ppm}) 166.9,160.7,137.3,135.9,135.4,135.3,130.4,129.5,128.4,127.1,126.6$, 124.3, 121.4, 115.7, 55.1, 51.3, 51.1, 30.2, 28.6, 28.0, 21.3; HRMS (ESI-TOF) m/z: $[\mathrm{M}+\mathrm{H}]^{+}$Calcd for $\mathrm{C}_{31} \mathrm{H}_{43} \mathrm{~N}_{4} \mathrm{O}_{2}$ 503.3381; Found: 503.3380.

$N^{2}, N^{3}$-Di-tert-butyl-5-(tert-butylamino)-1-(4-chlorophenyl)-4-phenyl-1H-pyrrole2,3-dicarboxamide $(6 \mathrm{~h})$ :

White solid, yield: $0.434 \mathrm{~g}(83 \%)$, ethylacetate/petroleum ether $=1: 15, \mathrm{mp} \mathrm{248-}$ $249{ }^{\circ} \mathrm{C} .{ }^{1} \mathrm{H} \mathrm{NMR}\left(\mathrm{CDCl}_{3}, 400 \mathrm{MHz}\right) \delta(\mathrm{ppm}) 8.63$ (s, 1H), 7.41-7.21 (m, 9H), 5.03 (s, $1 \mathrm{H}), 2.98(\mathrm{~s}, 1 \mathrm{H}), 1.34-0.50(\mathrm{~m}, 27 \mathrm{H}) ;{ }^{13} \mathrm{C}\left\{{ }^{1} \mathrm{H}\right\} \mathrm{NMR}\left(\mathrm{CDCl}_{3}, 100 \mathrm{MHz}\right) \delta(\mathrm{ppm})$ 
$166.5,160.3,137.2,135.1,134.8,133.2,130.2$, 128.8, 128.7, 128.5, 126.9, 124.4, 121.6, 116.8, 55.1, 51.4, 51.2, 30.1, 28.6, 28.0; HRMS (ESI-TOF) m/z: $[\mathrm{M}+\mathrm{H}]^{+}$ Calcd for $\mathrm{C}_{30} \mathrm{H}_{40} \mathrm{ClN}_{4} \mathrm{O}_{2}$ 523.2834; Found: 523.2835.

$N^{2}, N^{3}$-Di-tert-butyl-5-(tert-butylamino)-1-(2-chlorophenyl)-4-phenyl-1H-pyrrole-

\section{2,3-dicarboxamide (6i):}

White solid, yield: $0.319 \mathrm{~g}(61 \%)$, ethylacetate/petroleum ether $=1: 12, \mathrm{mp} \mathrm{211-}$ $212{ }^{\circ} \mathrm{C} .{ }^{1} \mathrm{H} \mathrm{NMR}\left(\mathrm{CDCl}_{3}, 400 \mathrm{MHz}\right) \delta(\mathrm{ppm}) 8.73$ (s, 1H), 7.51-7.27 (m, 9H), 5.07 (s, $1 \mathrm{H}), 2.86(\mathrm{~s}, 1 \mathrm{H}), 1.33-0.52(\mathrm{~m}, 27 \mathrm{H}) ;{ }^{13} \mathrm{C}\left\{{ }^{1} \mathrm{H}\right\} \mathrm{NMR}\left(\mathrm{CDCl}_{3}, 100 \mathrm{MHz}\right) \delta(\mathrm{ppm})$ $166.6,160.2$, 136.7, 135.0, 134.9, 132.2, 130.5, 129.9, 129.8, 128.9, 128.4, 126.9, 126.8, 125.0, 120.8, 116.8, 54.6, 51.4, 50.9, 30.2, 28.6, 28.0; HRMS (ESI-TOF) m/z: $[\mathrm{M}+\mathrm{H}]^{+}$Calcd for $\mathrm{C}_{30} \mathrm{H}_{40} \mathrm{ClN}_{4} \mathrm{O}_{2}$ 523.2834; Found: 523.2834.

$N^{2}, N^{3}$-Di-tert-butyl-5-(tert-butylamino)-1-(3,5-dichlorophenyl)-4-phenyl-1H-pyrr ole-2,3-dicarboxamide $(6 \mathbf{j})$ :

White solid, yield: $0.434 \mathrm{~g}(78 \%)$, ethylacetate/petroleum ether $=1: 15, \mathrm{mp} 232-$ $233{ }^{\circ} \mathrm{C} .{ }^{1} \mathrm{H} \mathrm{NMR}\left(\mathrm{CDCl}_{3}, 400 \mathrm{MHz}\right) \delta(\mathrm{ppm}) 8.62$ (s, 1H), 7.39-7.17 (m, 8H), $5.03(\mathrm{~s}$, $1 \mathrm{H}), 3.00(\mathrm{~s}, 1 \mathrm{H}), 1.35-0.50(\mathrm{~m}, 27 \mathrm{H}) ;{ }^{13} \mathrm{C}\left\{{ }^{1} \mathrm{H}\right\} \mathrm{NMR}\left(\mathrm{CDCl}_{3}, 100 \mathrm{MHz}\right) \delta(\mathrm{ppm})$ $166.5,160.3,139.7,135.1,134.7,134.1,130.2$, 129.4, 128.6, 127.8, 127.6, 126.9, 125.8, 124.5, 121.6, 117.0, 55.2, 51.4, 51.2, 30.1, 28.6, 28.0; HRMS (ESI-TOF) m/z: $[\mathrm{M}+\mathrm{H}]^{+}$Calcd for $\mathrm{C}_{30} \mathrm{H}_{39} \mathrm{Cl}_{2} \mathrm{~N}_{4} \mathrm{O}_{2}$ 557.2445; Found: 557.2444 .

$N^{2}, N^{3}$-Di-tert-butyl-5-(tert-butylamino)-1-(3,4-dichlorophenyl)-4-phenyl-1H-pyrr ole-2,3-dicarboxamide (6k):

White solid, yield: $0.412 \mathrm{~g}(74 \%)$, ethylacetate/petroleum ether $=1: 13, \mathrm{mp} 208-$ $209{ }^{\circ} \mathrm{C} .{ }^{1} \mathrm{H} \mathrm{NMR}\left(\mathrm{CDCl}_{3}, 400 \mathrm{MHz}\right) \delta(\mathrm{ppm}) 8.71(\mathrm{~s}, 1 \mathrm{H}), 7.50-7.13(\mathrm{~m}, 8 \mathrm{H}), 5.01(\mathrm{~s}$, $1 \mathrm{H}), 2.95(\mathrm{~s}, 1 \mathrm{H}), 1.36-0.51(\mathrm{~m}, 27 \mathrm{H}) ;{ }^{13} \mathrm{C}\left\{{ }^{1} \mathrm{H}\right\} \mathrm{NMR}\left(\mathrm{CDCl}_{3}, 100 \mathrm{MHz}\right) \delta(\mathrm{ppm})$ 166.3 , 160.1, 138.0, 135.0, 134.5, 132.2, 131.3, 130.1, 130.0, 129.4, 128.6, 127.1, 127.0, 124.5, 121.7, 117.6, 55.2, 51.4, 51.2, 30.1, 28.6, 28.0; HRMS (ESI-TOF) m/z: $[\mathrm{M}+\mathrm{H}]^{+}$Calcd for $\mathrm{C}_{30} \mathrm{H}_{39} \mathrm{Cl}_{2} \mathrm{~N}_{4} \mathrm{O}_{2}$ 557.2445; Found: 557.2444.

$N^{2}$-((3s,5s,7s)-Adamantan-1-yl)- $N^{3}$-(tert-butyl)-5-(tert-butylamino)-1-(4-chlorophe nyl)-4-phenyl-1H-pyrrole-2,3-dicarboxamide (61):

White solid, yield: $0.469 \mathrm{~g}(78 \%)$, ethylacetate/petroleum ether $=1: 15, \mathrm{mp} 252-$ 
$253{ }^{\circ} \mathrm{C} .{ }^{1} \mathrm{H}$ NMR $\left(\mathrm{CDCl}_{3}, 400 \mathrm{MHz}\right) \delta(\mathrm{ppm}) 8.47$ (s, 1H), 7.41-7.20 (m, 9H), 5.02 (s, 1H), $2.97(\mathrm{~s}, 1 \mathrm{H}), 2.02-0.49(\mathrm{~m}, 33 \mathrm{H}) ;{ }^{13} \mathrm{C}\left\{{ }^{1} \mathrm{H}\right\} \mathrm{NMR}\left(\mathrm{CDCl}_{3}, 100 \mathrm{MHz}\right) \delta(\mathrm{ppm})$ $166.5,160.0,137.2,135.1,134.9,133.2,130.2,128.8,128.7,128.5,126.9,124.5$, 121.6, 116.9, 55.1, 52.0, 51.4, 41.4, 36.4, 30.1, 29.4, 28.0; HRMS (ESI-TOF) m/z: [M $+\mathrm{H}]^{+}$Calcd for $\mathrm{C}_{36} \mathrm{H}_{46} \mathrm{ClN}_{4} \mathrm{O}_{2}$ 601.3304; Found: 601.3304 .

$N^{2}$-((3s,5s,7s)-Adamantan-1-yl)- $N^{3}$-(tert-butyl)-5-(tert-butylamino)-1,4-diphenyl-1 $H$-pyrrole-2,3-dicarboxamide $(6 \mathrm{~m})$ :

White solid, yield: $0.425 \mathrm{~g}(75 \%)$, ethylacetate/petroleum ether $=1: 14, \mathrm{mp} 277-$ $278{ }^{\circ} \mathrm{C} .{ }^{1} \mathrm{H}$ NMR $\left(\mathrm{CDCl}_{3}, 400 \mathrm{MHz}\right) \delta(\mathrm{ppm}) 8.35$ (s, 1H), 7.46-7.26 (m, 10H), 5.09 $(\mathrm{s}, 1 \mathrm{H}), 3.09(\mathrm{~s}, 1 \mathrm{H}), 2.01-0.47(\mathrm{~m}, 33 \mathrm{H}) ;{ }^{13} \mathrm{C}\left\{{ }^{1} \mathrm{H}\right\} \mathrm{NMR}\left(\mathrm{CDCl}_{3}, 100 \mathrm{MHz}\right) \delta(\mathrm{ppm})$ $166.7,160.3,138.5,135.3,135.2,130.4,128.7,128.4,127.5,127.4,126.6,124.4$, 121.4, 116.0, 55.0, 51.9, 51.3, 41.4, 36.4, 30.1, 29.4, 29.4, 28.1; HRMS (ESI-TOF) m/z: $[\mathrm{M}+\mathrm{H}]^{+}$Calcd for $\mathrm{C}_{36} \mathrm{H}_{47} \mathrm{~N}_{4} \mathrm{O}_{2}$ 567.3694; Found: 567.3692 .

$N^{2}$-((3s,5s,7s)-Adamantan-1-yl)-1-(4-bromophenyl)- $N^{3}$-(tert-butyl)-5-(tert-butyla mino)-4-phenyl-1H-pyrrole-2,3-dicarboxamide (6n):

White solid, yield: 0.445g (69\%), ethylacetate/petroleum ether $=1: 15, \mathrm{mp} \mathrm{269-}$ $270{ }^{\circ} \mathrm{C} .{ }^{1} \mathrm{H}$ NMR $\left(\mathrm{CDCl}_{3}, 400 \mathrm{MHz}\right) \delta(\mathrm{ppm}) 8.47$ (s, 1H), 7.56-7.15 (m, 9H), 5.02 (s, 1H), $2.97(\mathrm{~s}, 1 \mathrm{H}), 2.02-0.49(\mathrm{~m}, 33 \mathrm{H}) ;{ }^{13} \mathrm{C}\left\{{ }^{1} \mathrm{H}\right\} \mathrm{NMR}\left(\mathrm{CDCl}_{3}, 100 \mathrm{MHz}\right) \delta(\mathrm{ppm})$ $166.5,160.0$, 137.7, 135.0, 134.8, 131.8, 130.2, 129.0, 128.5, 126.9, 124.4, 121.6, 121.2, 116.9, 55.2, 52.0, 51.4, 41.4, 36.4, 30.1, 29.4, 28.0; HRMS (ESI-TOF) m/z: [M $+\mathrm{H}]^{+}$Calcd for $\mathrm{C}_{36} \mathrm{H}_{46} \mathrm{BrN}_{4} \mathrm{O}_{2}$ 645.2799; Found: 645.2798.

$N^{2}$-((3s,5s,7s)-Adamantan-1-yl)- $N^{3}$-(tert-butyl)-5-(tert-butylamino)-1-(3-chlorophe nyl)-4-phenyl-1H-pyrrole-2,3-dicarboxamide (6o):

White solid, yield: $0.433 \mathrm{~g}(72 \%)$, ethylacetate/petroleum ether $=1: 12, \mathrm{mp} \mathrm{254-}$ $255^{\circ} \mathrm{C} .{ }^{1} \mathrm{H} \mathrm{NMR}\left(\mathrm{CDCl}_{3}, 400 \mathrm{MHz}\right) \delta(\mathrm{ppm}) 8.45$ (s, 1H), 7.39-7.17 (m, 9H), 5.03 (s, $1 \mathrm{H}), 3.00(\mathrm{~s}, 1 \mathrm{H}), 2.03-0.49(\mathrm{~m}, 33 \mathrm{H}) ;{ }^{13} \mathrm{C}\left\{{ }^{1} \mathrm{H}\right\} \mathrm{NMR}\left(\mathrm{CDCl}_{3}, 100 \mathrm{MHz}\right) \delta(\mathrm{ppm})$ $166.5,160.0$, 139.7, 135.1, 134.8, 134.1, 130.2, 129.4, 128.5, 127.8, 127.6, 126.9, $125.8,124.6,121.6,117.0,55.1,52.0,51.4,41.3,36.4,30.1,29.4,28.0$; HRMS (ESI-TOF) m/z: $[\mathrm{M}+\mathrm{H}]^{+}$Calcd for $\mathrm{C}_{36} \mathrm{H}_{46} \mathrm{ClN}_{4} \mathrm{O}_{2}$ 601.3304; Found: 601.3303. $N^{2}$-((3s,5s,7s)-Adamantan-1-yl)- $N^{3}$-(tert-butyl)-5-(tert-butylamino)-1,4-bis(4-chlor 


\section{ophenyl)-1H-pyrrole-2,3-dicarboxamide (6p):}

White solid, yield: $0.464 \mathrm{~g}(73 \%)$, ethylacetate/petroleum ether $=1: 13, \mathrm{mp} \mathrm{286-}$ $287{ }^{\circ} \mathrm{C} .{ }^{1} \mathrm{H}$ NMR $\left(\mathrm{CDCl}_{3}, 400 \mathrm{MHz}\right) \delta(\mathrm{ppm}) 8.18(\mathrm{~s}, 1 \mathrm{H}), 7.42-7.18(\mathrm{~m}, 8 \mathrm{H}), 5.09(\mathrm{~s}$, 1H), $2.96(\mathrm{~s}, 1 \mathrm{H}), 2.01-0.51(\mathrm{~m}, 33 \mathrm{H}) ;{ }^{13} \mathrm{C}\left\{{ }^{1} \mathrm{H}\right\} \mathrm{NMR}\left(\mathrm{CDCl}_{3}, 100 \mathrm{MHz}\right) \delta(\mathrm{ppm})$ $166.4,159.9,136.9,135.0,133.4,133.3,132.6,131.4,129.0,128.6,128.5,124.5$, 121.5, 115.4, 55.4, 52.1, 51.6, 41.4, 36.4, 30.2, 29.4, 28.2; HRMS (ESI-TOF) m/z: [M $+\mathrm{H}]^{+}$Calcd for $\mathrm{C}_{36} \mathrm{H}_{45} \mathrm{Cl}_{2} \mathrm{~N}_{4} \mathrm{O}_{2}$ 635.2914; Found: 635.2913.

$N^{2}, N^{3}-\operatorname{Di}((3 s, 5 s, 7 s)$-adamantan-1-yl)-5-(((3s,5s,7s)-adamantan-1-yl)amino)-1,4-bis (4-chlorophenyl)-1H-pyrrole-2,3-dicarboxamide (6q):

White solid, yield: $0.601 \mathrm{~g}(76 \%)$, ethylacetate/petroleum ether $=1: 12, \mathrm{mp} 304$ $305{ }^{\circ} \mathrm{C} .{ }^{1} \mathrm{H} \mathrm{NMR}\left(\mathrm{CDCl}_{3}, 400 \mathrm{MHz}\right) \delta(\mathrm{ppm}) 8.11(\mathrm{~s}, 1 \mathrm{H}), 7.42-7.18(\mathrm{~m}, 8 \mathrm{H}), 4.94(\mathrm{~s}$, $1 \mathrm{H}), 2.93(\mathrm{~s}, 1 \mathrm{H}), 2.02-0.94(\mathrm{~m}, 45 \mathrm{H}) ;{ }^{13} \mathrm{C}\left\{{ }^{1} \mathrm{H}\right\} \mathrm{NMR}\left(\mathrm{CDCl}_{3}, 100 \mathrm{MHz}\right) \delta(\mathrm{ppm})$ $166.3,160.0,137.0,134.1,133.5,133.4,132.5,131.5,129.0,128.7,128.6,124.3$, 121.6, 115.4, 55.7, 52.2, 52.1, 43.6, 42.5, 41.4, 41.0, 36.4, 36.2, 35.8, 29.5, 29.2; HRMS (ESI-TOF) m/z: $[\mathrm{M}+\mathrm{H}]^{+}$Calcd for $\mathrm{C}_{48} \mathrm{H}_{57} \mathrm{Cl}_{2} \mathrm{~N}_{4} \mathrm{O}_{2}$ 791.3853; Found: 791.3853.

$N^{2}-((3 s, 5 s, 7 s)-A d a m a n t a n-1-y l)-1-(4-b r o m o p h e n y l)-N^{3}$-octyl-5-(octylamino)-4-ph enyl-1H-pyrrole-2,3-dicarboxamide (6r):

White solid, yield: $0.606 \mathrm{~g}(80 \%)$, ethylacetate/petroleum ether $=1: 15, \mathrm{mp} 185$ $186{ }^{\circ} \mathrm{C} .{ }^{1} \mathrm{H} \mathrm{NMR}\left(\mathrm{CDCl}_{3}, 400 \mathrm{MHz}\right) \delta(\mathrm{ppm}) 8.67$ (s, 1H), 7.56-7.13 (m, 9H), 5.11 (s, $1 \mathrm{H}), 3.02(\mathrm{~s}, 1 \mathrm{H}), 2.04-0.45(\mathrm{~m}, 49 \mathrm{H}) ;{ }^{13} \mathrm{C}\left\{{ }^{1} \mathrm{H}\right\} \mathrm{NMR}\left(\mathrm{CDCl}_{3}, 100 \mathrm{MHz}\right) \delta(\mathrm{ppm})$ 166.3, 159.4, 138.1, 135.2, 135.1, 131.7, 130.7, 129.3, 128.6, 127.0, 124.5, 121.4, 121.2 , 117.2, 59.2, 56.5, 55.6, 52.3, 52.0, 41.3, 36.4, 31.4, 31.3, 29.4, 29.3, 27.8; HRMS (ESI-TOF) m/z: $[\mathrm{M}+\mathrm{H}]^{+}$Calcd for $\mathrm{C}_{44} \mathrm{H}_{62} \mathrm{BrN}_{4} \mathrm{O}_{2}$ 757.4051; Found: 757.4044 .

$N^{2}$-((3s,5s,7s)-Adamantan-1-yl)-4-(4-chlorophenyl)- $N^{3}$-octyl-5-(octylamino)-1-phe nyl-1H-pyrrole-2,3-dicarboxamide (6s):

Light yellow oil; Yield: $0.535 \mathrm{~g}(75 \%)$, ethylacetate/petroleum ether $=1: 12, \mathrm{mp} 121-$ $122{ }^{\circ} \mathrm{C} .{ }^{1} \mathrm{H}$ NMR (DMSO- $\left.d_{6}, 400 \mathrm{MHz}\right) \delta(\mathrm{ppm}) 8.02$ (s, 1H), 7.38-7.14 (m, 9H), $3.46(\mathrm{~s}, 1 \mathrm{H}), 2.43(\mathrm{~s}, 1 \mathrm{H}), 1.10-0.33(\mathrm{~m}, 49 \mathrm{H}) ;{ }^{13} \mathrm{C}\left\{{ }^{1} \mathrm{H}\right\}$ NMR (DMSO- $d_{6}, 100 \mathrm{MHz}$ ) 
$\delta(\mathrm{ppm}) 165.0,160.8,138.1,134.7,134.1,132.1,130.6,128.2,127.5,127.2,124.4$ 120.6, 119.8, 117.1, 39.7, 39.5, 39.3, 31.3, 31.2, 31.1, 30.8, 28.9, 28.4, 28.1; HRMS (ESI-TOF) m/z: [M + H] $]^{+}$Calcd for $\mathrm{C}_{44} \mathrm{H}_{62} \mathrm{ClN}_{4} \mathrm{O}_{2}$ 713.4556; Found: 713.4555 .

$N^{2}, N^{3}$-Di $((3 s, 5 s, 7 s)$-adamantan-1-yl)-5-(((3s,5s,7s)-adamantan-1-yl)amino)-4-phe nyl-1-(p-tolyl)-1H-pyrrole-2,3-dicarboxamide (6t):

White solid, yield: $0.486 \mathrm{~g}(66 \%)$, ethylacetate/petroleum ether $=1: 15, \mathrm{mp} 186-$ $187{ }^{\circ} \mathrm{C} .{ }^{1} \mathrm{H} \mathrm{NMR}\left(\mathrm{CDCl}_{3}, 400 \mathrm{MHz}\right) \delta(\mathrm{ppm}) 8.27$ (s, 1H), 7.40-7.12 (m, 8H), 4.93 (s, $1 \mathrm{H}), 3.08(\mathrm{~s}, 1 \mathrm{H}), 2.40(\mathrm{~s}, 3 \mathrm{H}), 2.01-0.93(\mathrm{~m}, 45 \mathrm{H}) ;{ }^{13} \mathrm{C}\left\{{ }^{1} \mathrm{H}\right\} \mathrm{NMR}\left(\mathrm{CDCl}_{3}, 100 \mathrm{MHz}\right)$ $\delta(\mathrm{ppm}) 166.6,160.5,137.2,135.9,135.4,134.5,130.5,129.5,128.3,127.1,126.5$, 124.1, 121.5, 115.7, 55.3, 52.0, 51.9, 43.4, 41.4, 40.9, 29.6, 29.5, 29.3, 21.3; HRMS (ESI-TOF) m/z: [M + H] $]^{+}$Calcd for $\mathrm{C}_{49} \mathrm{H}_{61} \mathrm{~N}_{4} \mathrm{O}_{2}$ 737.4789; Found: 737.4786.

$N^{2}$-((3s,5s,7s)-Adamantan-1-yl)-1-(4-fluorophenyl)- $N^{3}$-octyl-5-(octylamino)-4-phe nyl-1H-pyrrole-2,3-dicarboxamide (6u):

White solid, yield: $0.502 \mathrm{~g}(72 \%)$, ethylacetate/petroleum ether $=1: 12, \mathrm{mp} 134-$ $135{ }^{\circ} \mathrm{C} .{ }^{1} \mathrm{H}$ NMR (DMSO- $\left.d_{6}, 400 \mathrm{MHz}\right) \delta(\mathrm{ppm}) 8.35(\mathrm{~s}, 1 \mathrm{H}), 7.41-7.31(\mathrm{~m}, 9 \mathrm{H})$, $6.84(\mathrm{~s}, 1 \mathrm{H}), 3.57(\mathrm{~s}, 1 \mathrm{H}), 2.03-0.47(\mathrm{~m}, 49 \mathrm{H}) ;{ }^{13} \mathrm{C}\left\{{ }^{1} \mathrm{H}\right\}$ NMR (DMSO- $\left.d_{6}, 100 \mathrm{MHz}\right)$ $\delta(\mathrm{ppm}) 165.5,160.9\left(\mathrm{~d}, J_{\mathrm{C}-\mathrm{F}}=242.1 \mathrm{~Hz}\right), 159.9,135.3,134.9\left(\mathrm{~d}, J_{\mathrm{C}-\mathrm{F}}=2.7 \mathrm{~Hz}\right)$, $134.5,130.5,130.0\left(\mathrm{~d}, J_{\mathrm{C}-\mathrm{F}}=8.8 \mathrm{~Hz}\right), 127.7,126.1,123.8,120.4,118.4,114.8\left(\mathrm{~d}, J_{\mathrm{C}-\mathrm{F}}\right.$ $=22.7 \mathrm{~Hz}), 57.7,56.2,54.5,51.1,40.8,36.0,31.3,31.2,30.8,28.9,28.7,28.1$; HRMS (ESI-TOF) m/z: $[\mathrm{M}+\mathrm{H}]^{+}$Calcd for $\mathrm{C}_{44} \mathrm{H}_{62} \mathrm{FN}_{4} \mathrm{O}_{2}$ 697.4851; Found: 697.4848 .

$N^{2}$-((3s,5s,7s)-Adamantan-1-yl)- $N^{3}$-(tert-butyl)-5-(tert-butylamino)-1-(4-chlorophe nyl)-4-(4-fluorophenyl)-1H-pyrrole-2,3-dicarboxamide (6v):

White solid, yield: $0.483 \mathrm{~g}(78 \%)$, ethylacetate/petroleum ether $=1: 14, \mathrm{mp} 262-$ $263{ }^{\circ} \mathrm{C} .{ }^{1} \mathrm{H} \mathrm{NMR}\left(\mathrm{CDCl}_{3}, 400 \mathrm{MHz}\right) \delta(\mathrm{ppm}) 8.27$ (s, 1H), 7.42-7.09 (m, 8H), $5.07(\mathrm{~s}$, 1H), $2.94(\mathrm{~s}, 1 \mathrm{H}), 2.01-0.50(\mathrm{~m}, 33 \mathrm{H}) ;{ }^{13} \mathrm{C}\left\{{ }^{1} \mathrm{H}\right\} \mathrm{NMR}\left(\mathrm{CDCl}_{3}, 100 \mathrm{MHz}\right) \delta(\mathrm{ppm})$ $166.5,161.8\left(\mathrm{~d}, J_{\mathrm{C}-\mathrm{F}}=245.1 \mathrm{~Hz}\right), 160.0, \quad 137.0,135.1,133.4,131.7\left(\mathrm{~d}, J_{\mathrm{C}-\mathrm{F}}=7.8\right.$ $\mathrm{Hz}), 130.9\left(\mathrm{~d}, J_{\mathrm{C}-\mathrm{F}}=3.0 \mathrm{~Hz}\right), 129.0,128.6,124.4,121.6,115.6,115.4\left(\mathrm{~d}, J_{\mathrm{C}-\mathrm{F}}=21.4\right.$ $\mathrm{Hz}$ ), 55.2, 52.1, 51.5, 41.4, 36.4, 30.2, 29.4, 28.2; HRMS (ESI-TOF) m/z: $[\mathrm{M}+\mathrm{H}]^{+}$ Calcd for $\mathrm{C}_{36} \mathrm{H}_{45} \mathrm{ClFN}_{4} \mathrm{O}_{2}$ 619.3210; Found: 619.3209. 
(Z)-5-Benzyl-1-(4-bromophenyl)-2-(tert-butylamino)-4-(tert-butylimino)-3-phenyl -4,5-dihydropyrrolo $[3,4-b]$ pyrrol-6(1H)-one $(7 a)$ :

Light yellow solid, yield: $0.443 \mathrm{~g}(76 \%)$, ethylacetate/petroleum ether $=1: 25, \mathrm{mp}$ 181-182 ${ }^{\circ} \mathrm{C} .{ }^{1} \mathrm{H} \mathrm{NMR}\left(\mathrm{CDCl}_{3}, 400 \mathrm{MHz}\right) \delta(\mathrm{ppm})$ 7.59-7.17 (m, 14H), $4.81(\mathrm{~s}, 2 \mathrm{H})$, $2.84(\mathrm{~s}, 1 \mathrm{H}), 1.04(\mathrm{~s}, 9 \mathrm{H}), 0.56(\mathrm{~s}, 9 \mathrm{H}) ;{ }^{13} \mathrm{C}\left\{{ }^{1} \mathrm{H}\right\} \mathrm{NMR}\left(\mathrm{CDCl}_{3}, 100 \mathrm{MHz}\right) \delta(\mathrm{ppm})$ $159.9,142.5,142.4,139.4,135.5,135.2$, 132.6, 131.6, 129.3, 129.0, 128.2, 128.0, $127.9,127.4,126.6,121.7,119.5,116.0,54.8,53.2,41.7,30.9,30.3$; HRMS (ESI-TOF) m/z: $[\mathrm{M}+\mathrm{H}]^{+}$Calcd for $\mathrm{C}_{33} \mathrm{H}_{36} \mathrm{BrN}_{4} \mathrm{O}$ 583.2067; Found: 583.2066.

(Z)-2-(tert-Butylamino)-4-(tert-butylimino)-1-(4-chlorophenyl)-5-cyclohexyl-3-ph enyl-4,5-dihydropyrrolo[3,4-b]pyrrol-6(1H)-one (7b):

Light yellow solid, yield: $0.430 \mathrm{~g}(81 \%)$, ethylacetate/petroleum ether $=1: 30, \mathrm{mp}$ 102-103 ${ }^{\circ} \mathrm{C} .{ }^{1} \mathrm{H}$ NMR $\left(\mathrm{CDCl}_{3}, 400 \mathrm{MHz}\right) \delta(\mathrm{ppm})$ 7.55-7.26 (m, 9H), 4.26-4.22 (m, $1 \mathrm{H}), 2.84(\mathrm{~s}, 1 \mathrm{H}), 2.24-0.56(\mathrm{~m}, 28 \mathrm{H}) ;{ }^{13} \mathrm{C}\left\{{ }^{1} \mathrm{H}\right\} \mathrm{NMR}\left(\mathrm{CDCl}_{3}, 100 \mathrm{MHz}\right) \delta(\mathrm{ppm})$ $160.4,142.2$, 135.5, 135.0, 133.5, 132.7, 129.1, 128.5, 128.2, 127.9, 125.7, 123.5, 118.7, 115.6, 54.7, 53.0, 50.8, 30.8, 30.3, 29.8, 26.6, 25.7; HRMS (ESI-TOF) m/z: [M $+\mathrm{H}]^{+}$Calcd for $\mathrm{C}_{32} \mathrm{H}_{40} \mathrm{ClN}_{4} \mathrm{O}$ 531.2885; Found: 531.2884.

(Z)-1-(4-Chlorophenyl)-5-cyclohexyl-2-(octylamino)-4-(octylimino)-3-phenyl-4,5dihydropyrrolo[3,4-b]pyrrol-6(1H)-one $(7 \mathrm{c})$ :

Bright yellow solid, yield: $0.502 \mathrm{~g}(78 \%)$, ethylacetate/petroleum ether $=1: 30, \mathrm{mp}$ 186-187 ${ }^{\circ} \mathrm{C} .{ }^{1} \mathrm{H}$ NMR $\left(\mathrm{CDCl}_{3}, 400 \mathrm{MHz}\right) \delta(\mathrm{ppm})$ 7.54-7.26 (m, 9H), 4.35-4.29 (m, $1 \mathrm{H}), 3.01(\mathrm{~s}, 1 \mathrm{H}), 1.78-0.58(\mathrm{~m}, 44 \mathrm{H}) ;{ }^{13} \mathrm{C}\left\{{ }^{1} \mathrm{H}\right\} \mathrm{NMR}\left(\mathrm{CDCl}_{3}, 100 \mathrm{MHz}\right) \delta(\mathrm{ppm})$ $160.3,142.4,141.3,135.8,135.1,133.6,133.1,129.4,128.5,128.2,127.9,123.4$, $118.7,115.6,58.7,57.0,56.6,56.0,50.4,31.8,31.4,30.6,29.9,29.4,26.6,25.6$; HRMS (ESI-TOF) m/z: $[\mathrm{M}+\mathrm{H}]^{+}$Calcd for $\mathrm{C}_{40} \mathrm{H}_{56} \mathrm{ClN}_{4} \mathrm{O}$ 643.4137; Found: 643.4135 .

(Z)-2-(tert-Butylamino)-4-(tert-butylimino)-5-cyclohexyl-1-(4-fluorophenyl)-3-phe nyl-4,5-dihydropyrrolo[3,4-b]pyrrol-6(1H)-one $(7 \mathrm{~d})$ :

Light yellow solid, yield: $0.427 \mathrm{~g}(83 \%)$, ethylacetate/petroleum ether $=1: 28, \mathrm{mp}$ 176-177 ${ }^{\circ} \mathrm{C} .{ }^{1} \mathrm{H}$ NMR $\left(\mathrm{CDCl}_{3}, 400 \mathrm{MHz}\right) \delta(\mathrm{ppm})$ 7.55-7.14 (m, 9H), 4.26-4.23 (m, $1 \mathrm{H}), 2.84(\mathrm{~s}, 1 \mathrm{H}), 2.26-0.55(\mathrm{~m}, 28 \mathrm{H}) ;{ }^{13} \mathrm{C}\left\{{ }^{1} \mathrm{H}\right\} \mathrm{NMR}\left(\mathrm{CDCl}_{3}, 100 \mathrm{MHz}\right) \delta(\mathrm{ppm})$ 
$161.8\left(\mathrm{~d}, J_{\mathrm{C}-\mathrm{F}}=164.2 \mathrm{~Hz}\right), 160.4,142.3\left(\mathrm{~d}, J_{\mathrm{C}-\mathrm{F}}=4.1 \mathrm{~Hz}\right), 135.6,132.7,132.3\left(\mathrm{~d}, J_{\mathrm{C}-\mathrm{F}}\right.$ $=1.6 \mathrm{~Hz}), 129.5\left(\mathrm{~d}, J_{\mathrm{C}-\mathrm{F}}=5.6 \mathrm{~Hz}\right), 128.1,127.9,127.8,118.4,115.4,115.2\left(\mathrm{~d}, J_{\mathrm{C}-\mathrm{F}}=\right.$ 15.2 Hz), 54.5, 53.0, 50.8, 30.8, 30.3, 29.8, 26.6, 25.6; HRMS (ESI-TOF) m/z: [M + $\mathrm{H}]^{+}$Calcd for $\mathrm{C}_{32} \mathrm{H}_{40} \mathrm{FN}_{4} \mathrm{O}$ 515.3181; Found: 515.3180.

(Z)-2-(tert-Butylamino)-4-(tert-butylimino)-5-cyclohexyl-1,3-diphenyl-4,5-dihydro pyrrolo[3,4-b]pyrrol-6(1H)-one $(7 \mathrm{e})$ :

Light yellow solid, yield: $0.398 \mathrm{~g}(80 \%)$, ethylacetate/petroleum ether $=1: 30, \mathrm{mp}$ 178-179 ${ }^{\circ} \mathrm{C} .{ }^{1} \mathrm{H}$ NMR $\left(\mathrm{CDCl}_{3}, 400 \mathrm{MHz}\right) \delta(\mathrm{ppm})$ 7.57-7.26 (m, 10H), 4.28-4.22 (m, $1 \mathrm{H}), 2.84(\mathrm{~s}, 1 \mathrm{H}), 2.28-0.53(\mathrm{~m}, 28 \mathrm{H}) ;{ }^{13} \mathrm{C}\left\{{ }^{1} \mathrm{H}\right\} \mathrm{NMR}\left(\mathrm{CDCl}_{3}, 100 \mathrm{MHz}\right) \delta(\mathrm{ppm})$ $160.4,142.5,136.4,135.8,132.8,128.3,128.1,127.9,127.8,127.8,127.7,118.4$, 115.3, 54.4, 53.0, 50.8, 30.8, 30.2, 29.9, 26.6, 25.7; HRMS (ESI-TOF) m/z: $[\mathrm{M}+\mathrm{H}]^{+}$ Calcd for $\mathrm{C}_{32} \mathrm{H}_{41} \mathrm{~N}_{4} \mathrm{O}$ 497.3275; Found: 497.3274.

(Z)-2-(tert-Butylamino)-4-(tert-butylimino)-1,3-bis(4-chlorophenyl)-5-cyclohexyl-

\section{4,5-dihydropyrrolo[3,4-b]pyrrol-6(1H)-one (7f):}

Light yellow solid, yield: $0.463 \mathrm{~g}(82 \%)$, ethylacetate/petroleum ether $=1: 30, \mathrm{mp}$ $178-179{ }^{\circ} \mathrm{C} .{ }^{1} \mathrm{H}$ NMR $\left(\mathrm{CDCl}_{3}, 400 \mathrm{MHz}\right) \delta(\mathrm{ppm})$ 7.53-7.24 (m, 8H), 4.26-4.20 (m, $1 \mathrm{H}), 2.76(\mathrm{~s}, 1 \mathrm{H}), 2.23-0.58(\mathrm{~m}, 28 \mathrm{H}) ;{ }^{13} \mathrm{C}\left\{{ }^{1} \mathrm{H}\right\} \mathrm{NMR}\left(\mathrm{CDCl}_{3}, 100 \mathrm{MHz}\right) \delta(\mathrm{ppm})$ 160.2 , 142.1, 142.0, 134.8, 134.1, 134.0, 133.9, 133.7, 129.0, 128.6, 128.4, 128.2, 118.6, 114.4, 54.8, 53.1, 50.9, 30.9, 30.4, 29.8, 26.6, 25.6; HRMS (ESI-TOF) m/z: [M

$+\mathrm{H}]^{+}$Calcd for $\mathrm{C}_{32} \mathrm{H}_{39} \mathrm{Cl}_{2} \mathrm{~N}_{4} \mathrm{O}$ 565.2495; Found: 565.2495.

(Z)-2-(tert-Butylamino)-4-(tert-butylimino)-3-(4-chlorophenyl)-5-cyclohexyl-1-(mtolyl)-4,5-dihydropyrrolo[3,4-b]pyrrol-6(1H)-one $(7 \mathrm{~g})$ :

Light yellow solid, yield: $0.409 \mathrm{~g}(75 \%)$, ethylacetate/petroleum ether $=1: 30, \mathrm{mp}$ 165-166 ${ }^{\circ} \mathrm{C} .{ }^{1} \mathrm{H}$ NMR $\left(\mathrm{CDCl}_{3}, 400 \mathrm{MHz}\right) \delta(\mathrm{ppm})$ 7.40-7.18 (m, 8H), 4.26-4.20 (m, $1 \mathrm{H}), 2.76(\mathrm{~s}, 1 \mathrm{H}), 2.40(\mathrm{~s}, 1 \mathrm{H}), 1.74-0.55(\mathrm{~m}, 28 \mathrm{H}) ;{ }^{13} \mathrm{C}\left\{{ }^{1} \mathrm{H}\right\} \mathrm{NMR}\left(\mathrm{CDCl}_{3}, 100 \mathrm{MHz}\right)$ $\delta(\mathrm{ppm}) 160.3,142.3,138.3,136.1,134.6,134.2,133.8,128.8,128.3,128.2,124.9$, 118.2, 113.9, 54.5, 53.0, 50.9, 30.9, 30.4, 29.9, 26.6, 25.7, 21.4; HRMS (ESI-TOF) $\mathrm{m} / \mathrm{z}:[\mathrm{M}+\mathrm{H}]^{+}$Calcd for $\mathrm{C}_{33} \mathrm{H}_{42} \mathrm{ClN}_{4} \mathrm{O}$ 545.3042; Found: 545.3041.

(Z)-2-(((3s,5s,7s)-Adamantan-1-yl)amino)-4-(((3s,5s,7s)-adamantan-1-yl)imino)-3 -(4-chlorophenyl)-5-cyclohexyl-1-(m-tolyl)-4,5-dihydropyrrolo[3,4-b]pyrrol-6(1H) 
-one (7h):

Light yellow solid, yield: $0.547 \mathrm{~g}(78 \%)$, ethylacetate/petroleum ether $=1: 25, \mathrm{mp}$ 145-147 ${ }^{\circ} \mathrm{C} .{ }^{1} \mathrm{H}$ NMR $\left(\mathrm{CDCl}_{3}, 400 \mathrm{MHz}\right) \delta(\mathrm{ppm})$ 7.42-7.18 (m, 8H), 4.27-4.21 (m, $1 \mathrm{H}), 2.73(\mathrm{~s}, 1 \mathrm{H}), 2.40-0.92(\mathrm{~m}, 43 \mathrm{H}) ;{ }^{13} \mathrm{C}\left\{{ }^{1} \mathrm{H}\right\} \mathrm{NMR}\left(\mathrm{CDCl}_{3}, 100 \mathrm{MHz}\right) \delta(\mathrm{ppm})$ $160.4,142.1,140.7,138.2,136.2,135.2,134.0,133.9,128.8,128.5,128.4,128.3$, $128.2,125.1,118.6,113.5,54.6,54.2,50.9,43.5,42.6,35.9,35.6,29.9,29.7,29.3$, 26.6, 25.7, 21.3; HRMS (ESI-TOF) m/z: $[\mathrm{M}+\mathrm{H}]^{+}$Calcd for $\mathrm{C}_{45} \mathrm{H}_{54} \mathrm{ClN}_{4} \mathrm{O}$ 701.3981; Found: 701.3980.

(E)-5-Butyl-2-(tert-butylamino)-4-(tert-butylimino)-1-(4-chlorophenyl)-3-phenyl4,5-dihydropyrrolo[3,4-b]pyrrol-6(1H)-one (7i):

Light yellow solid, yield: $0.434 \mathrm{~g}(86 \%)$, ethylacetate/petroleum ether $=1: 30, \mathrm{mp}$ 140-141 ${ }^{\circ} \mathrm{C} .{ }^{1} \mathrm{H}$ NMR $\left(\mathrm{CDCl}_{3}, 400 \mathrm{MHz}\right) \delta(\mathrm{ppm})$ 7.57-7.26 (m, 9H), 3.63-3.59 (m, $1 \mathrm{H}), 2.85(\mathrm{~s}, 1 \mathrm{H}), 1.54-0.57(\mathrm{~m}, 25 \mathrm{H}) ;{ }^{13} \mathrm{C}\left\{{ }^{1} \mathrm{H}\right\} \mathrm{NMR}\left(\mathrm{CDCl}_{3}, 100 \mathrm{MHz}\right) \delta(\mathrm{ppm})$ $160.1,142.6,142.2,135.4,135.0,133.5,132.6,129.0,128.5,128.2,128.0,127.8$, 119.2, 115.9, 54.8, 53.0, 38.0, 31.0, 30.9, 30.3, 20.2, 13.9; HRMS (ESI-TOF) m/z: [M $+\mathrm{H}]^{+}$Calcd for $\mathrm{C}_{30} \mathrm{H}_{38} \mathrm{ClN}_{4} \mathrm{O}$ 505.2729; Found: 505.2728.

(Z)-2-(((3s,5s,7s)-Adamantan-1-yl)amino)-4-(((3s,5s,7s)-adamantan-1-yl)imino)-5 -butyl-1-(4-chlorophenyl)-3-phenyl-4,5-dihydropyrrolo[3,4-b]pyrrol-6(1H)-one (7j):

Light yellow solid, yield: $0.535 \mathrm{~g}(81 \%), \mathrm{mp} 172-174{ }^{\circ} \mathrm{C} .{ }^{1} \mathrm{H} \mathrm{NMR}\left(\mathrm{CDCl}_{3}, 400 \mathrm{MHz}\right)$ $\delta(\mathrm{ppm}) 7.55-7.31(\mathrm{~m}, 9 \mathrm{H}), 3.63-3.60(\mathrm{~m}, 2 \mathrm{H}), 1.73(\mathrm{~s}, 1 \mathrm{H}), 1.56-0.88(\mathrm{~m}, 37 \mathrm{H}) ;{ }^{13} \mathrm{C}$ $\left\{{ }^{1} \mathrm{H}\right\} \mathrm{NMR}\left(\mathrm{CDCl}_{3}, 100 \mathrm{MHz}\right) \delta(\mathrm{ppm}) 160.2,142.0,141.1,136.2,135.1,133.5$, $132.5,129.1,128.5,128.1,119.5,115.5,54.9,54.1,43.5,42.6,35.8,35.6,30.9,29.6$, 29.3, 20.3, 13.9; HRMS (ESI-TOF) m/z: $[\mathrm{M}+\mathrm{H}]^{+}$Calcd for $\mathrm{C}_{42} \mathrm{H}_{50} \mathrm{ClN}_{4} \mathrm{O}$ 661.3668; Found: 661.3665 .

(E)-5-Butyl-2-(tert-butylamino)-4-(tert-butylimino)-3-phenyl-1-(4-(trifluoromethy l)phenyl)-4,5-dihydropyrrolo[3,4-b]pyrrol-6(1H)-one (7k):

Light yellow solid, yield: $0.431 \mathrm{~g}(80 \%)$, ethylacetate/petroleum ether $=1: 25, \mathrm{mp}$ 147-148 ${ }^{\circ} \mathrm{C} .{ }^{1} \mathrm{H}$ NMR $\left(\mathrm{CDCl}_{3}, 400 \mathrm{MHz}\right) \delta(\mathrm{ppm})$ 7.95-7.26 (m, 9H), 3.64-3.60 (m, 2H), $2.90(\mathrm{~s}, 1 \mathrm{H}), 1.56-0.54(\mathrm{~m}, 25 \mathrm{H}) ;{ }^{13} \mathrm{C}\left\{{ }^{1} \mathrm{H}\right\} \mathrm{NMR}\left(\mathrm{CDCl}_{3}, 100 \mathrm{MHz}\right) \delta(\mathrm{ppm})$ 
$160.1,142.5,142.1,136.9,135.3,132.6,131.0,130.8\left(\mathrm{~d}, J_{\mathrm{C}-\mathrm{F}}=32.6 \mathrm{~Hz}\right), 128.9$, 128.2, 128.0, 127.7, $124.9\left(\mathrm{q}, J_{\mathrm{C}-\mathrm{F}}=3.7 \mathrm{~Hz}\right), 124.3\left(\mathrm{q}, J_{\mathrm{C}-\mathrm{F}}=3.5 \mathrm{~Hz}\right), 123.8\left(\mathrm{~d}, J_{\mathrm{C}-\mathrm{F}}=\right.$ $270.8 \mathrm{~Hz}), 119.5,116.2,54.9,53.0,38.1,31.0,30.9,30.2,20.2,13.9$; HRMS (ESI-TOF) m/z: $[\mathrm{M}+\mathrm{H}]^{+}$Calcd for $\mathrm{C}_{31} \mathrm{H}_{38} \mathrm{~F}_{3} \mathrm{~N}_{4} \mathrm{O}$ 539.2992; Found: 539.2992. 
6. Copies of ${ }^{1} \mathrm{H}$ and ${ }^{13} \mathrm{C}$ NMR Spectrum

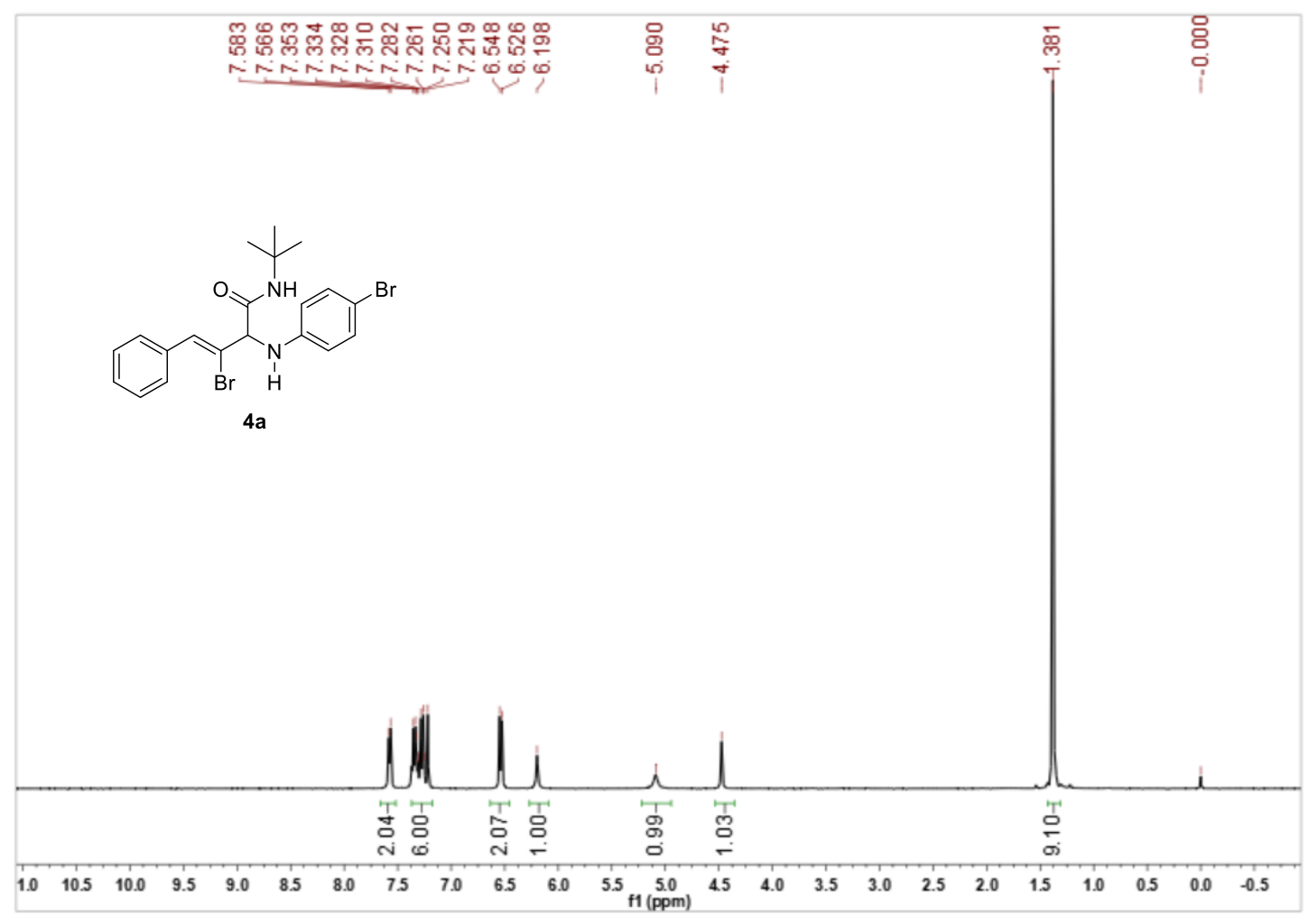

${ }^{1} \mathrm{H}$ NMR (400 MHz), $\mathrm{CDCl}_{3}$

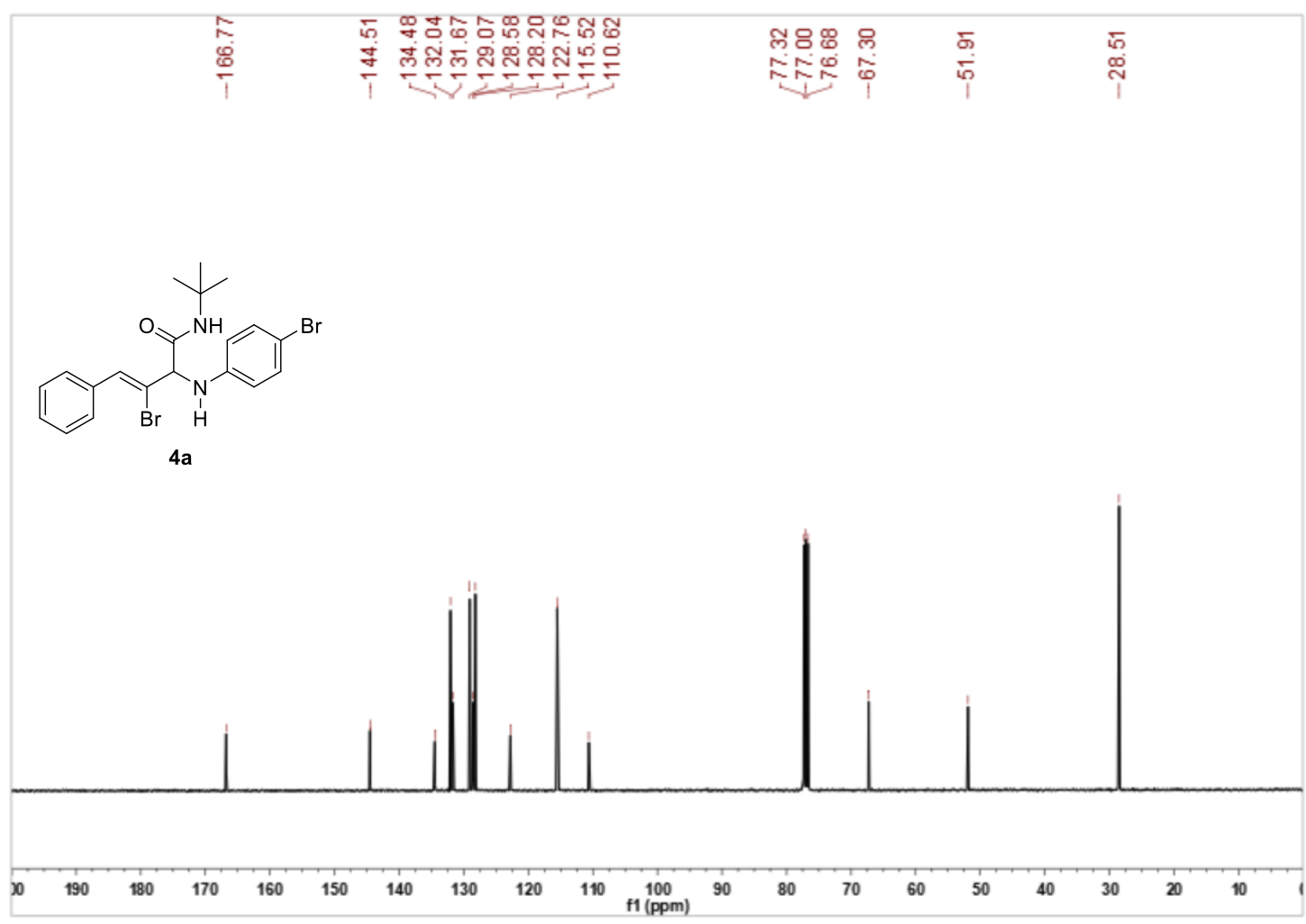

${ }^{13} \mathrm{C} \mathrm{NMR}(100 \mathrm{MHz}), \mathrm{CDCl}_{3}$

26 


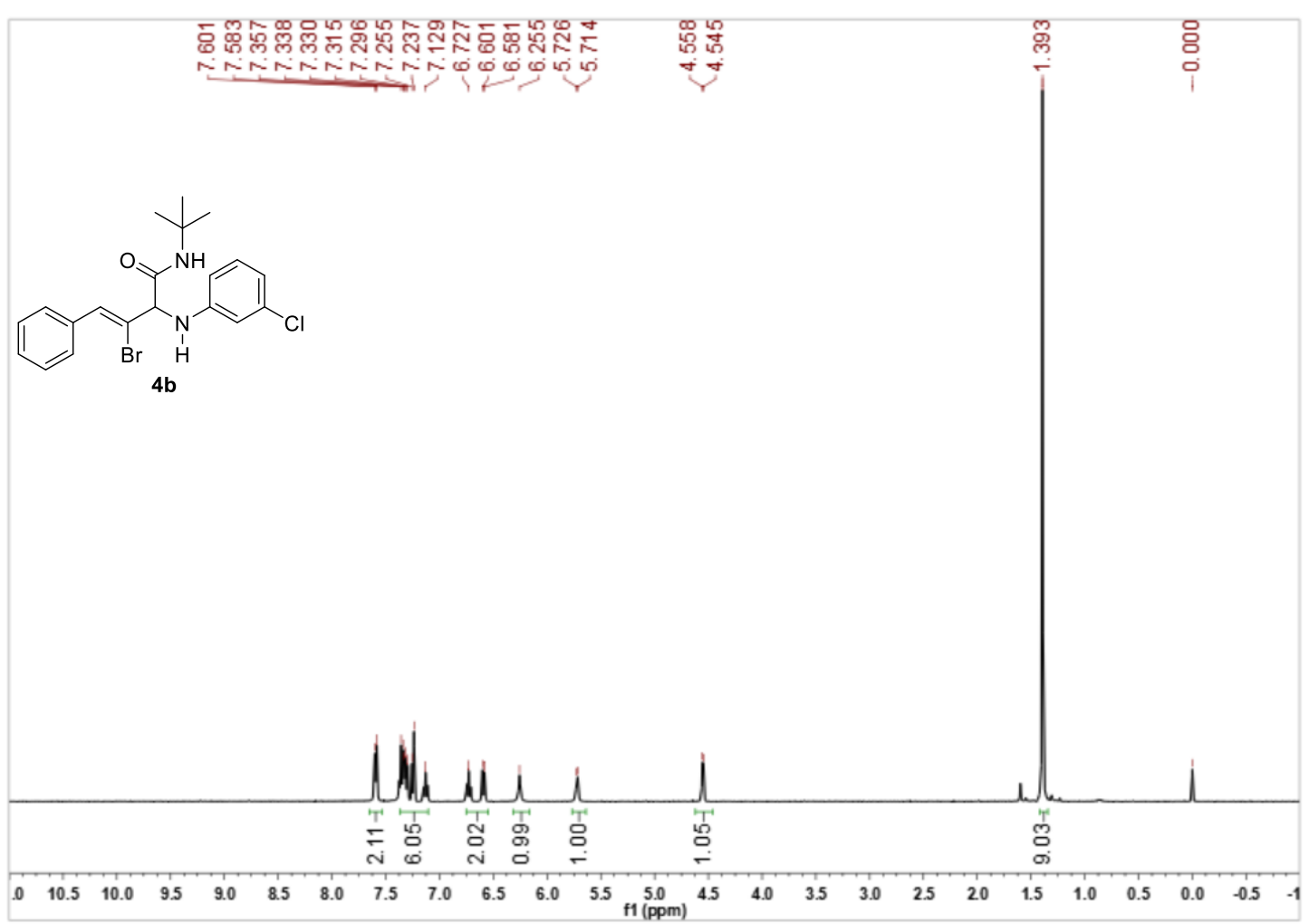

${ }^{1} \mathrm{H}$ NMR (400 MHz), $\mathrm{CDCl}_{3}$

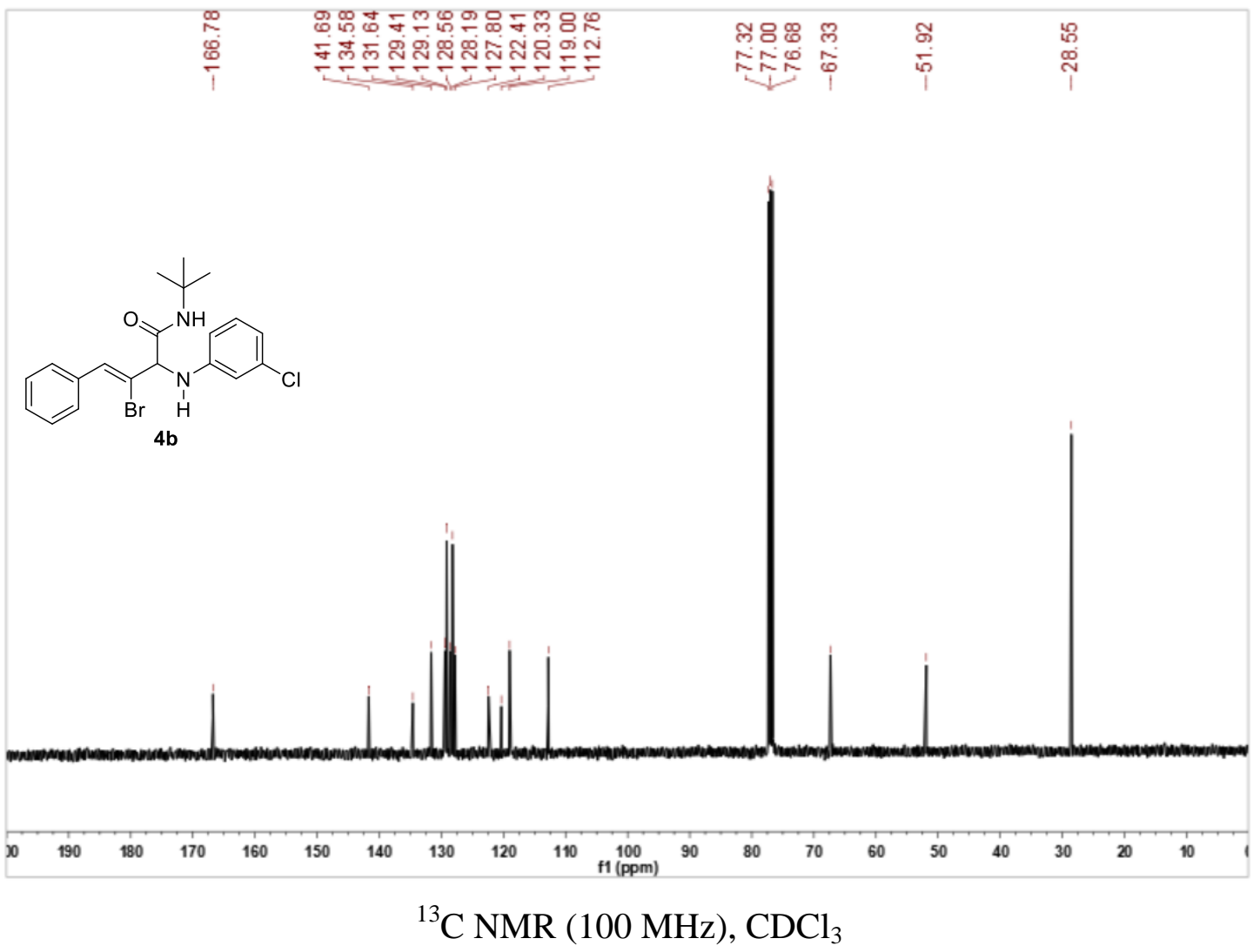




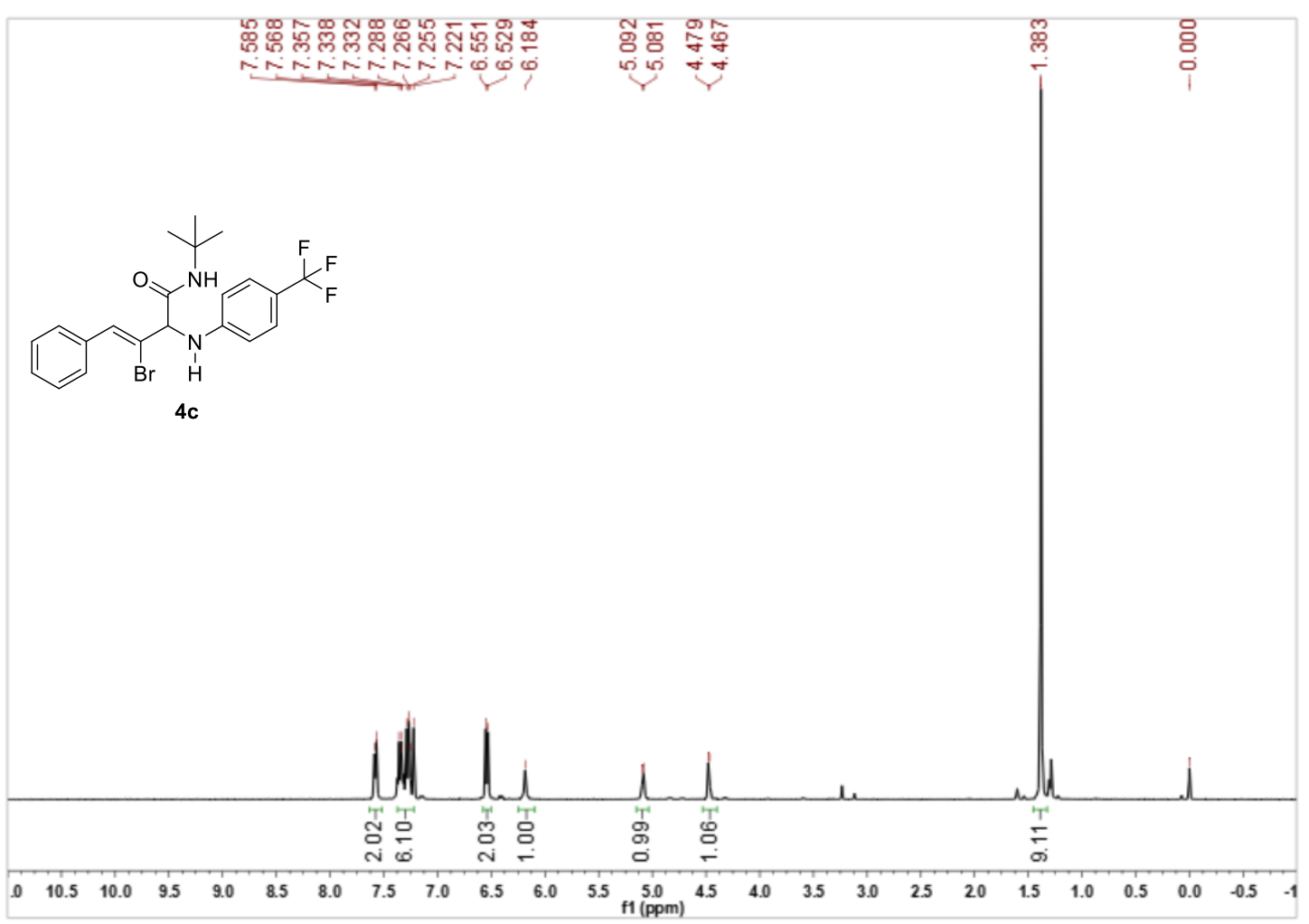

${ }^{1} \mathrm{H}$ NMR (400 MHz), $\mathrm{CDCl}_{3}$

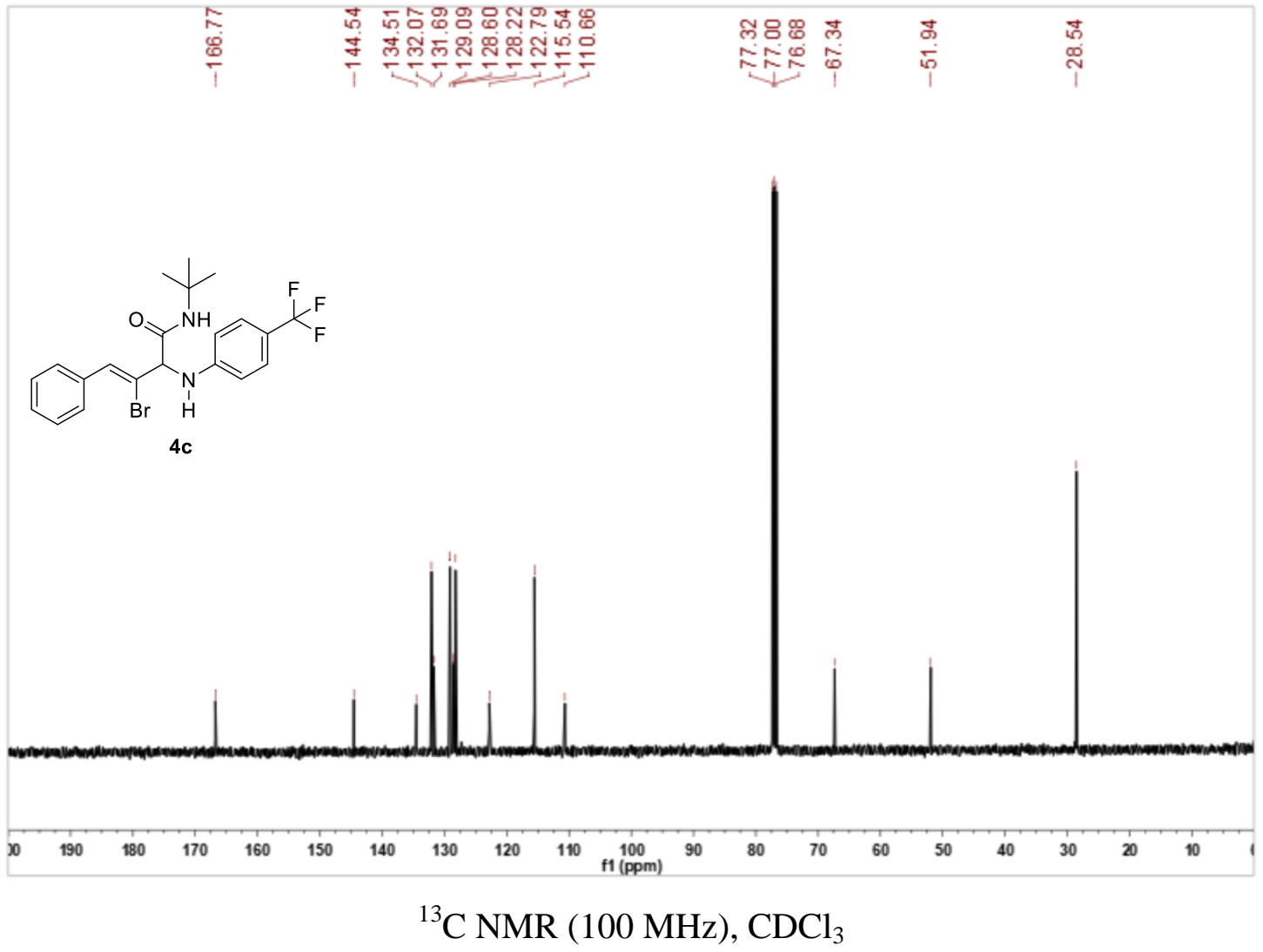




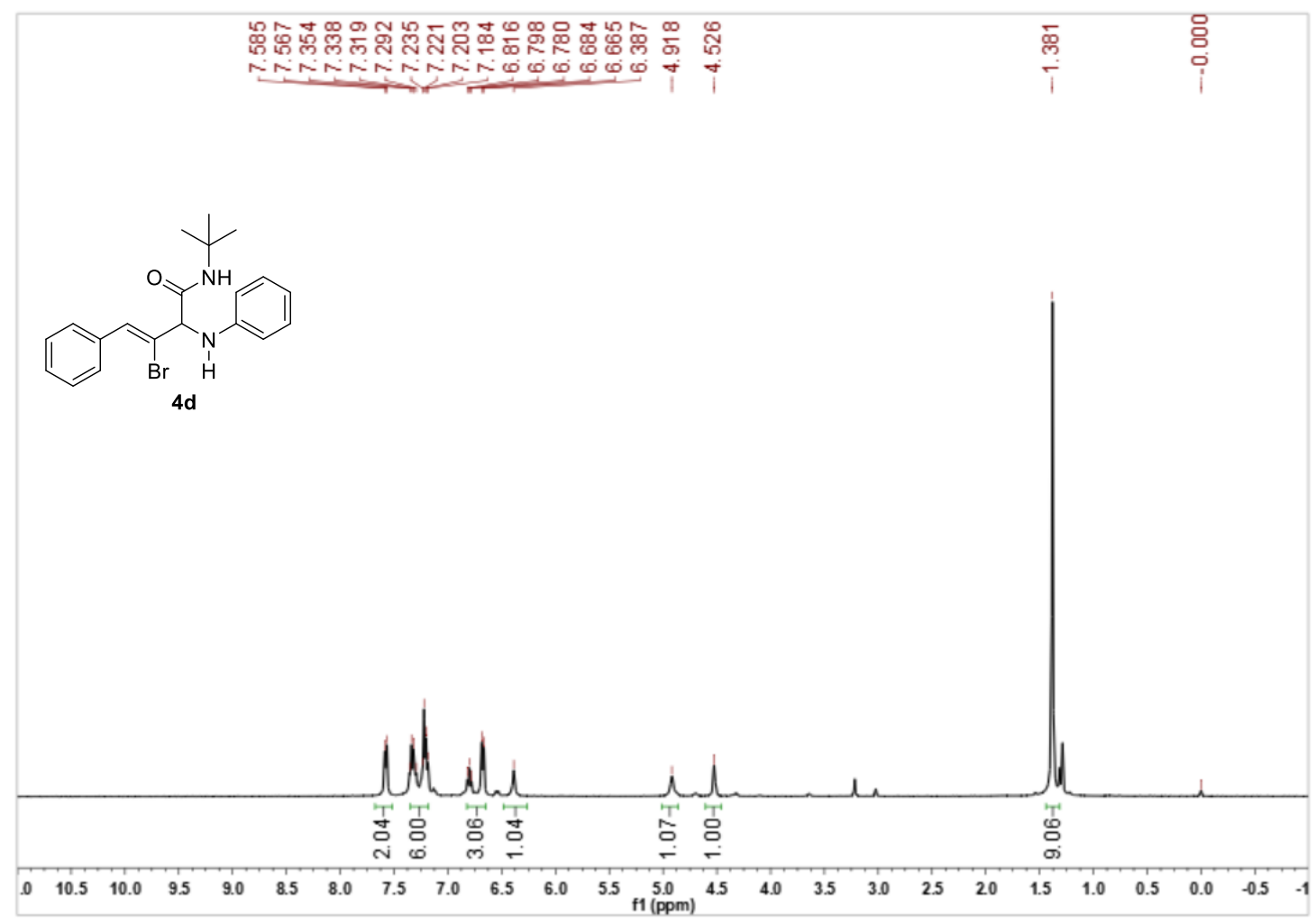

${ }^{1} \mathrm{H}$ NMR (400 MHz), $\mathrm{CDCl}_{3}$

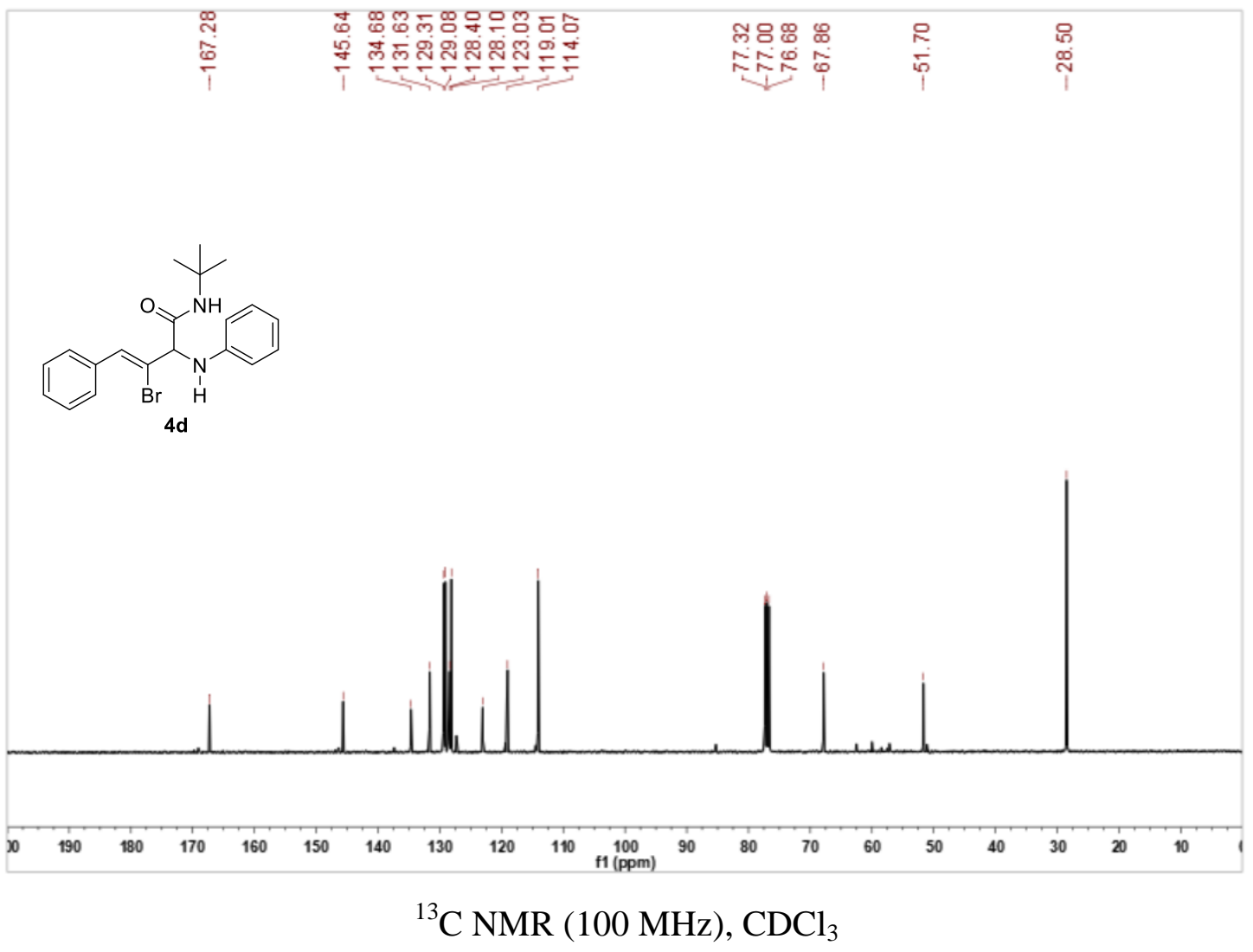




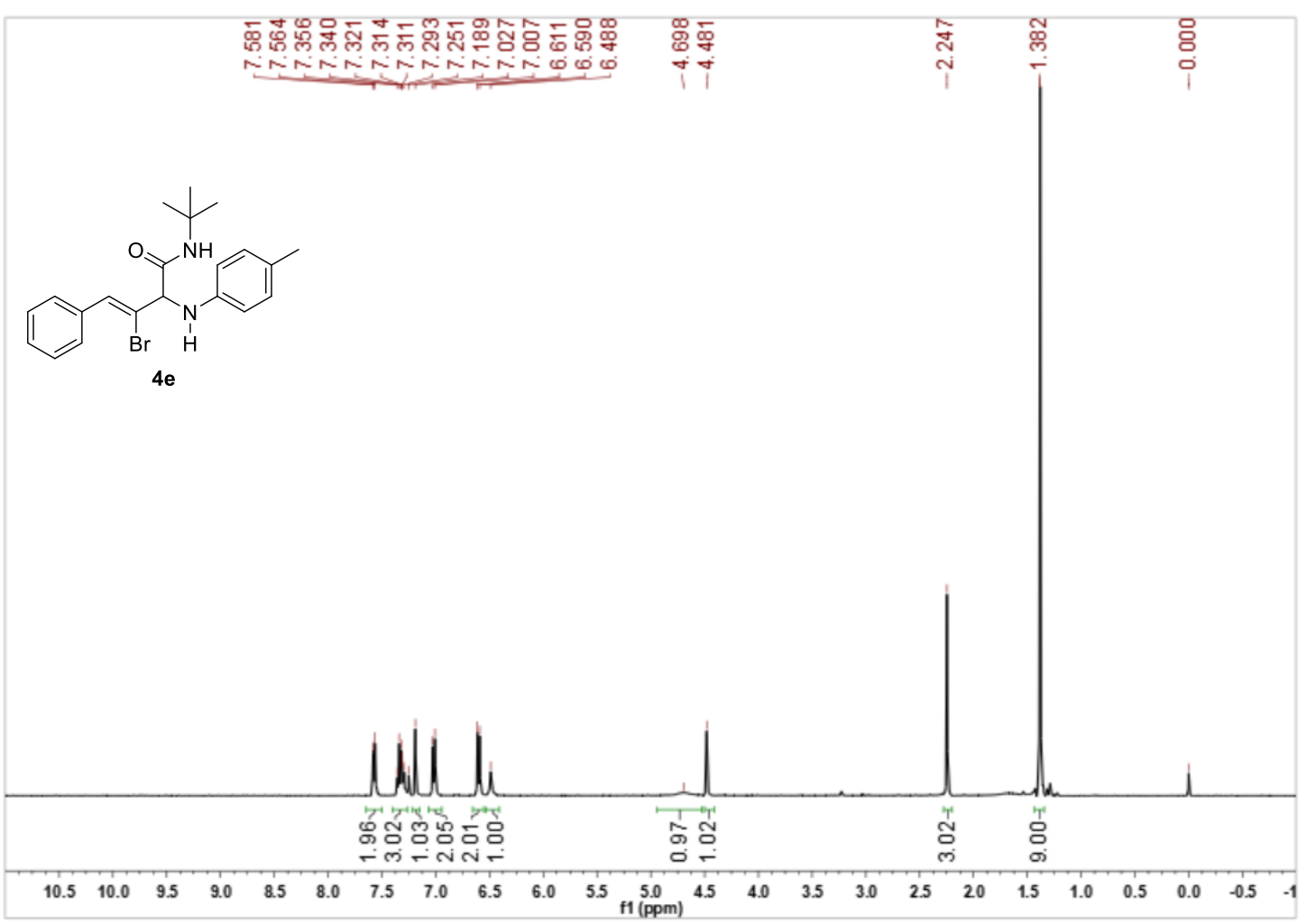

${ }^{1} \mathrm{H}$ NMR (400 MHz), $\mathrm{CDCl}_{3}$

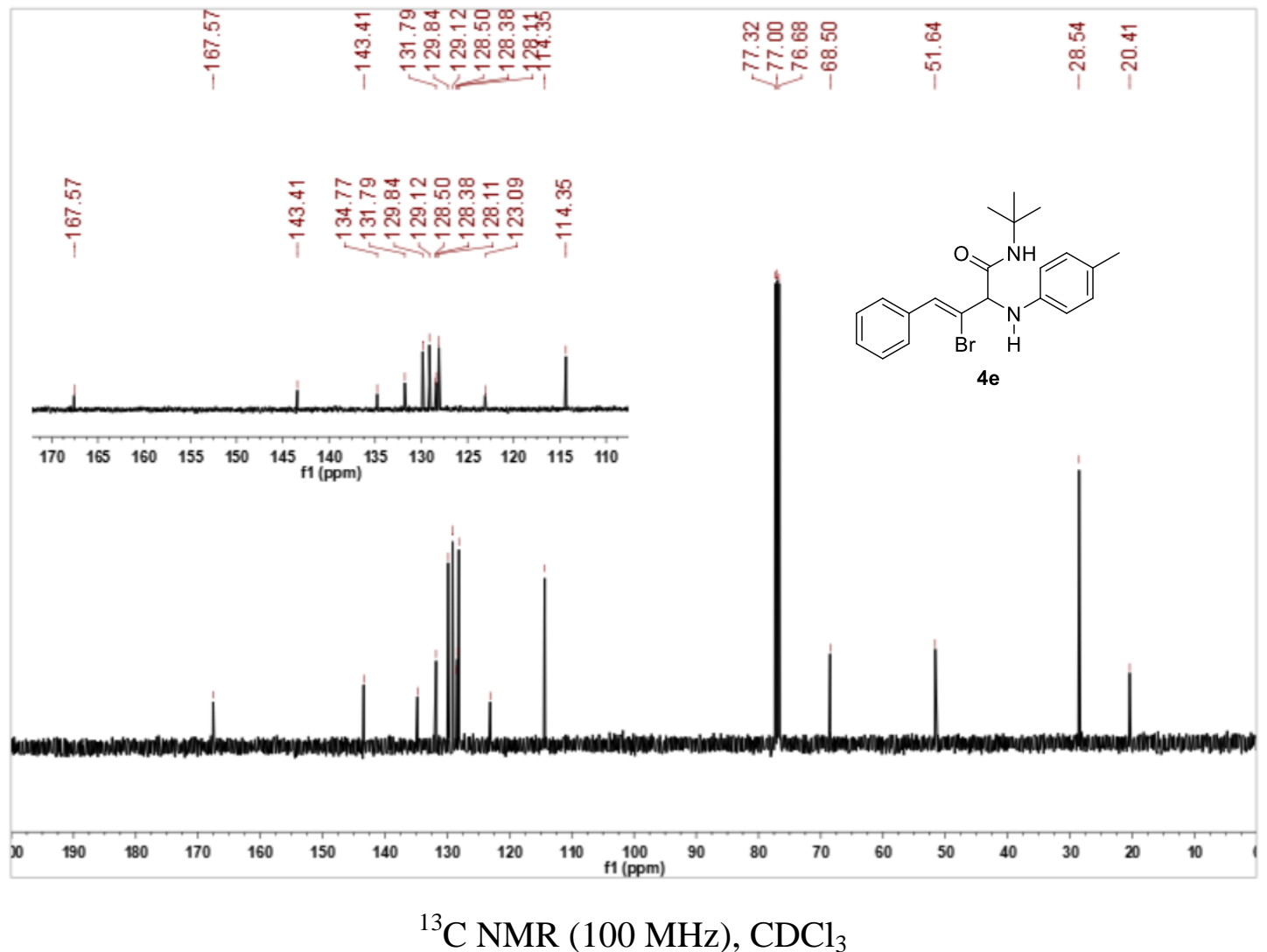

${ }^{13} \mathrm{C}$ NMR $(100 \mathrm{MHz}), \mathrm{CDCl}_{3}$ 


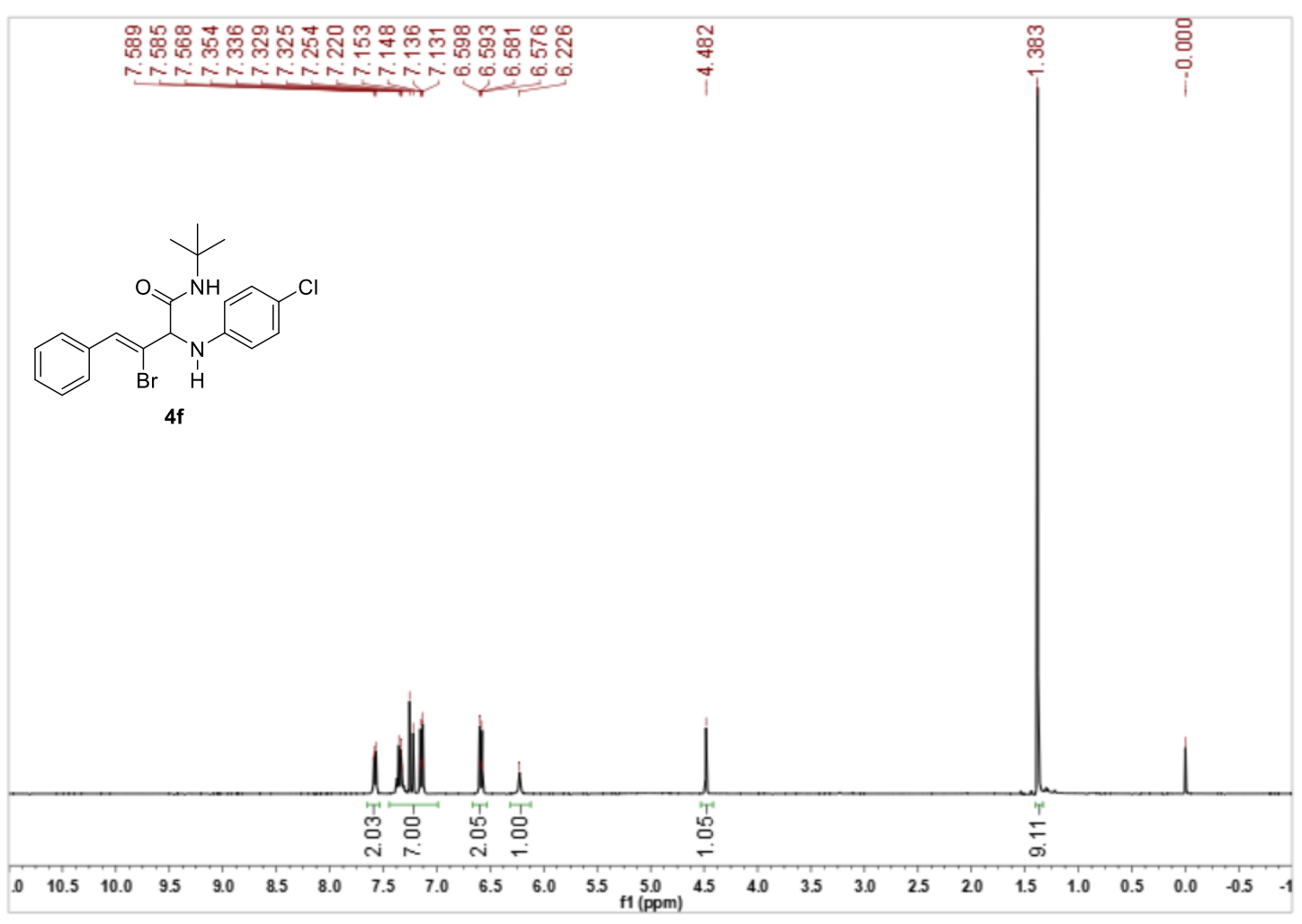

${ }^{1} \mathrm{H}$ NMR (400 MHz), $\mathrm{CDCl}_{3}$

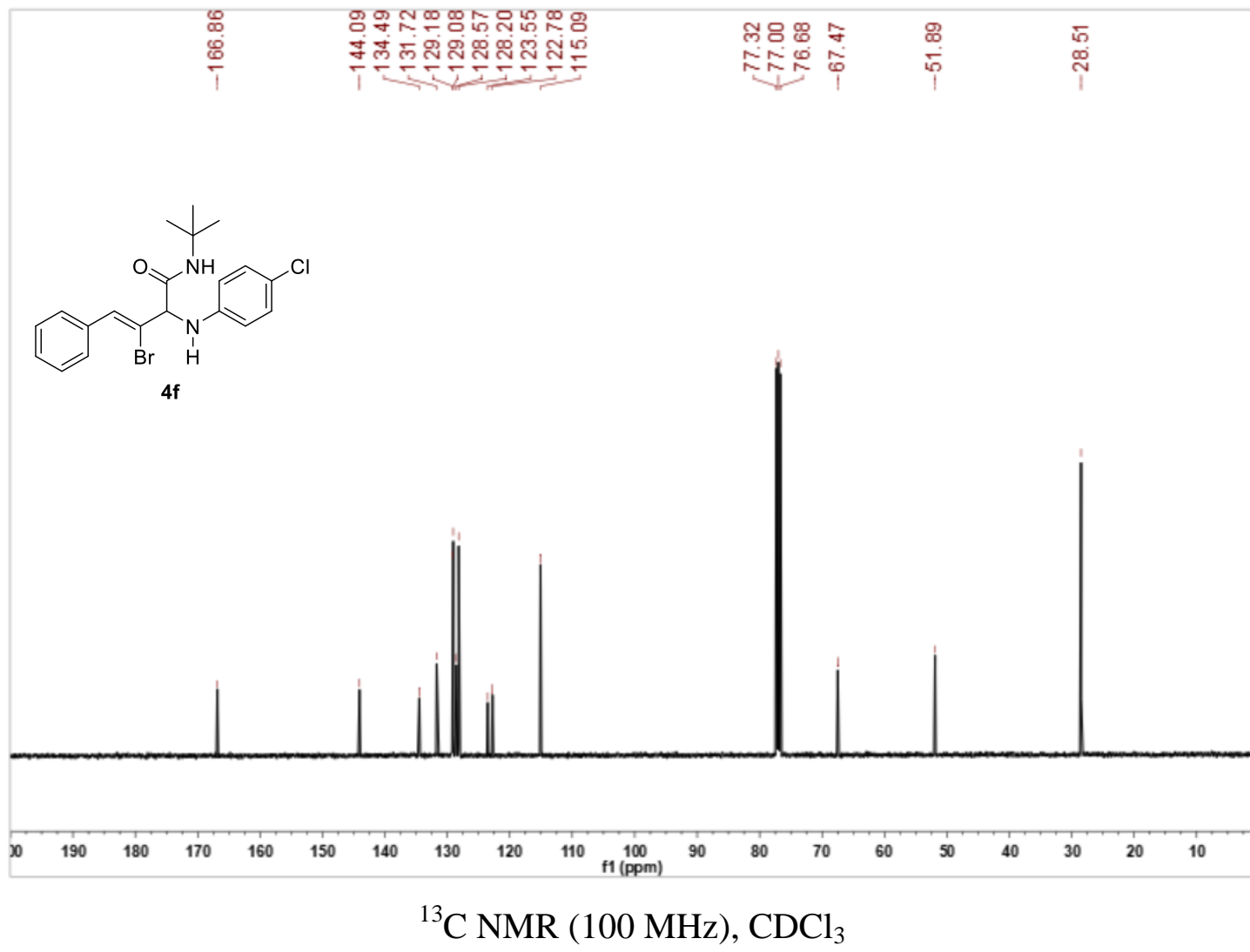




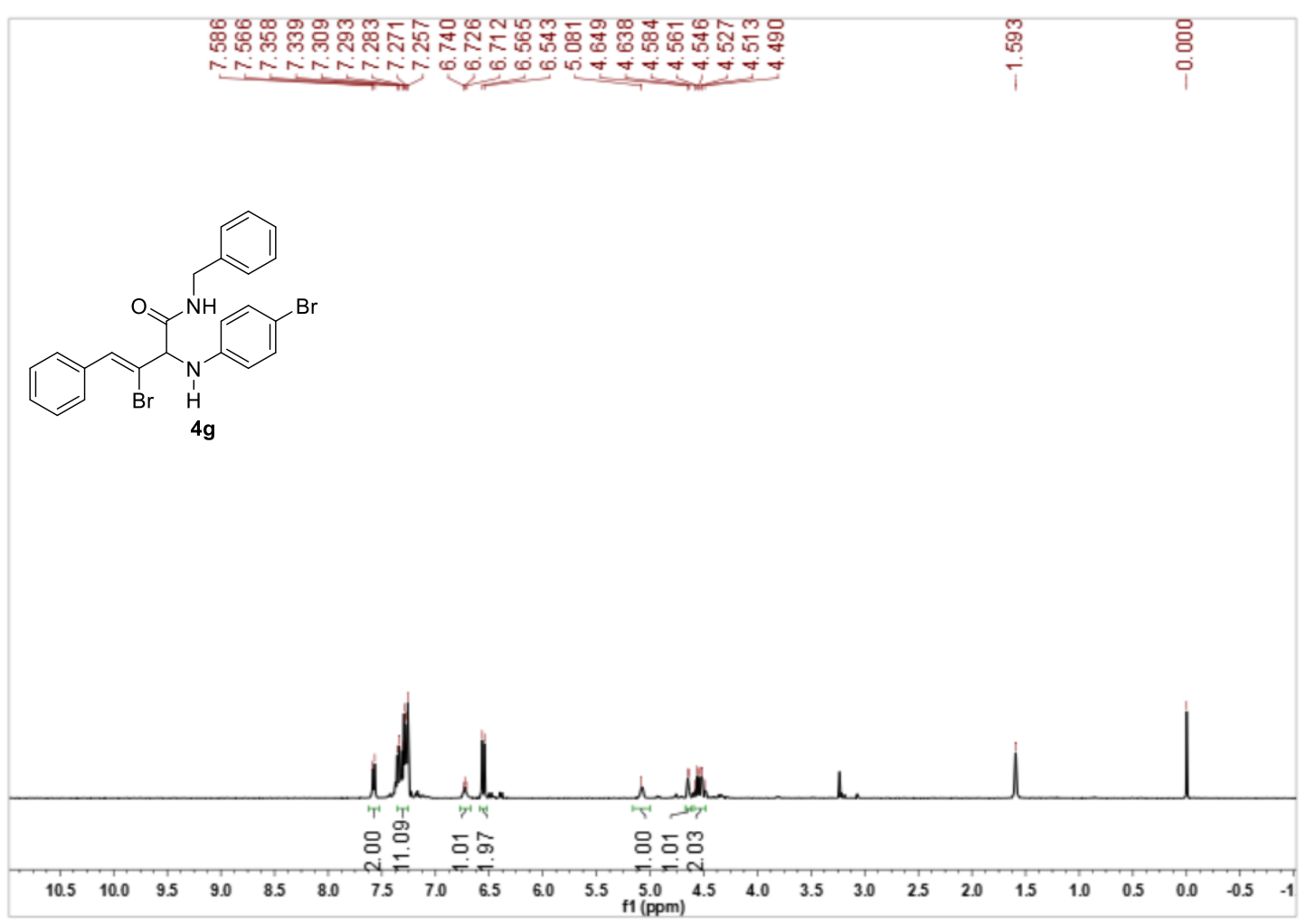

${ }^{1} \mathrm{H}$ NMR (400 MHz), $\mathrm{CDCl}_{3}$

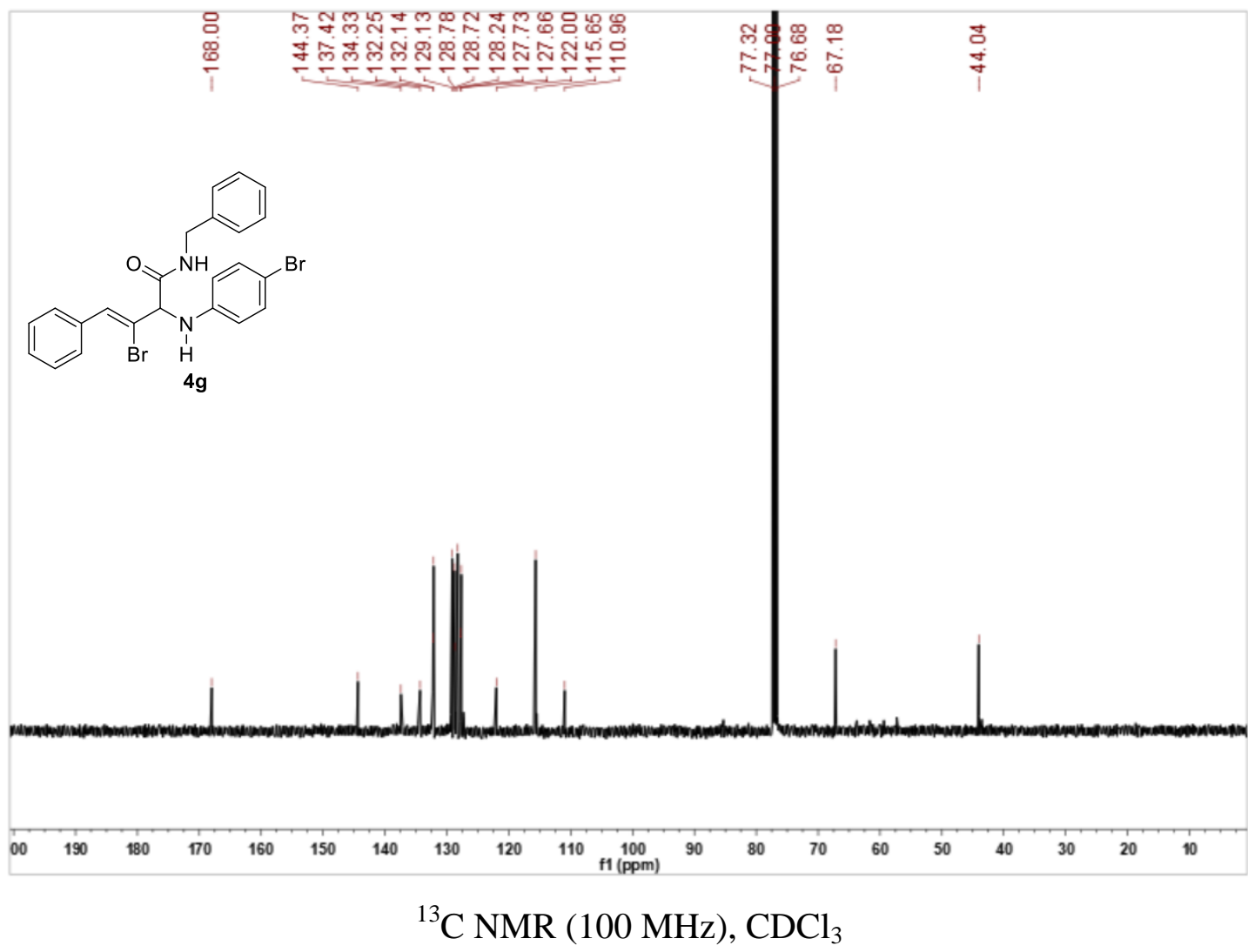




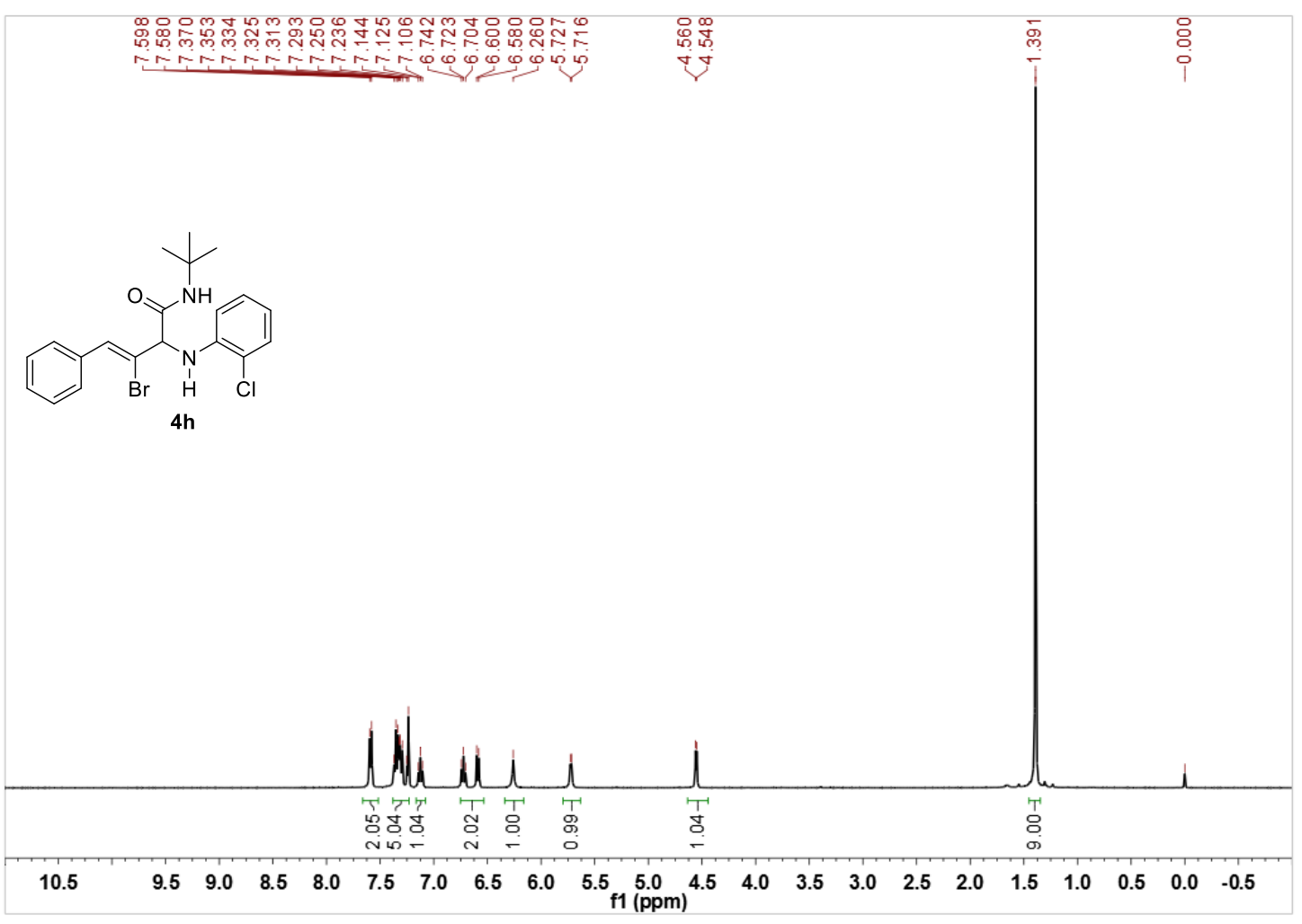

${ }^{1} \mathrm{H}$ NMR (400 MHz), $\mathrm{CDCl}_{3}$

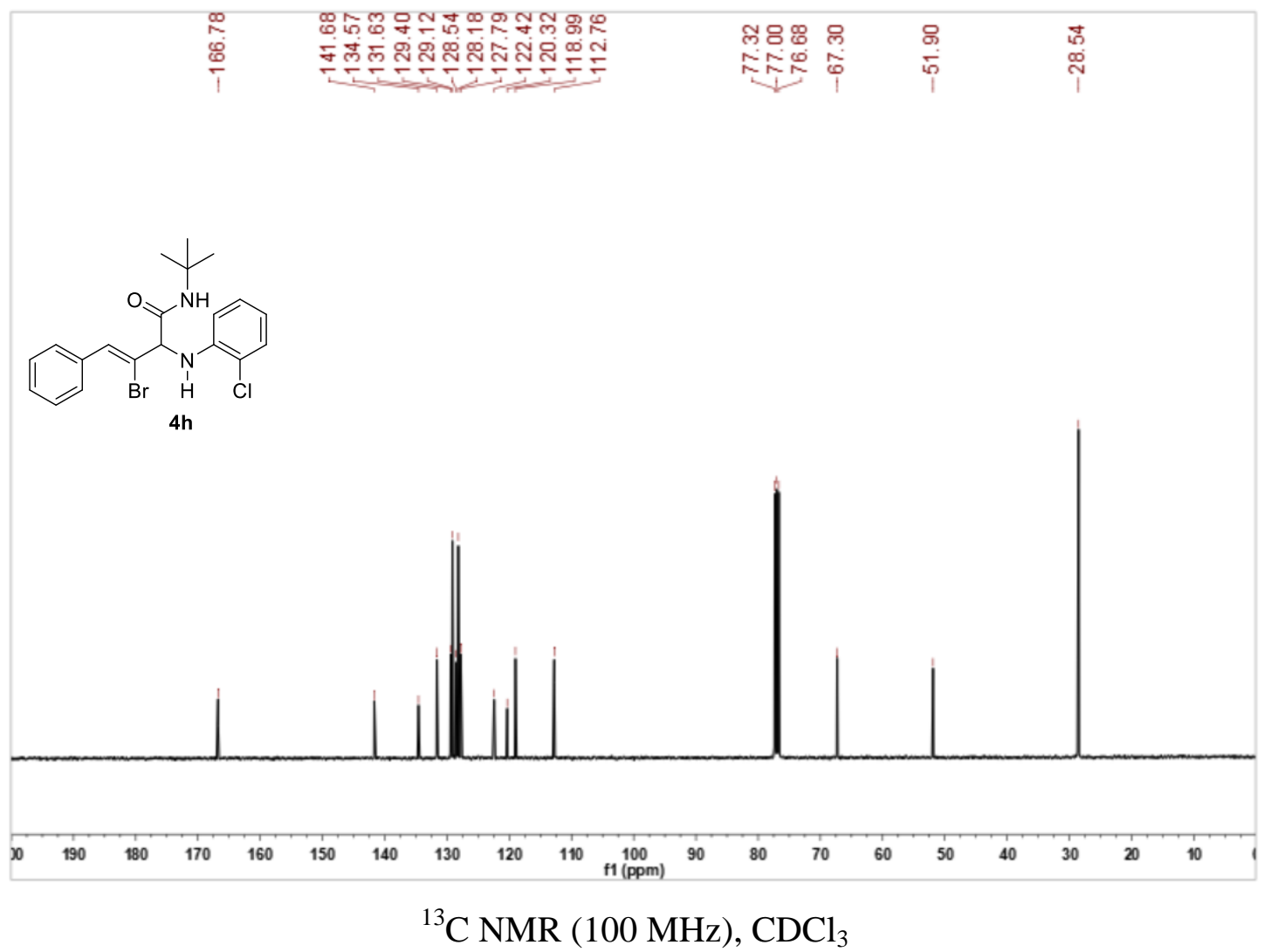




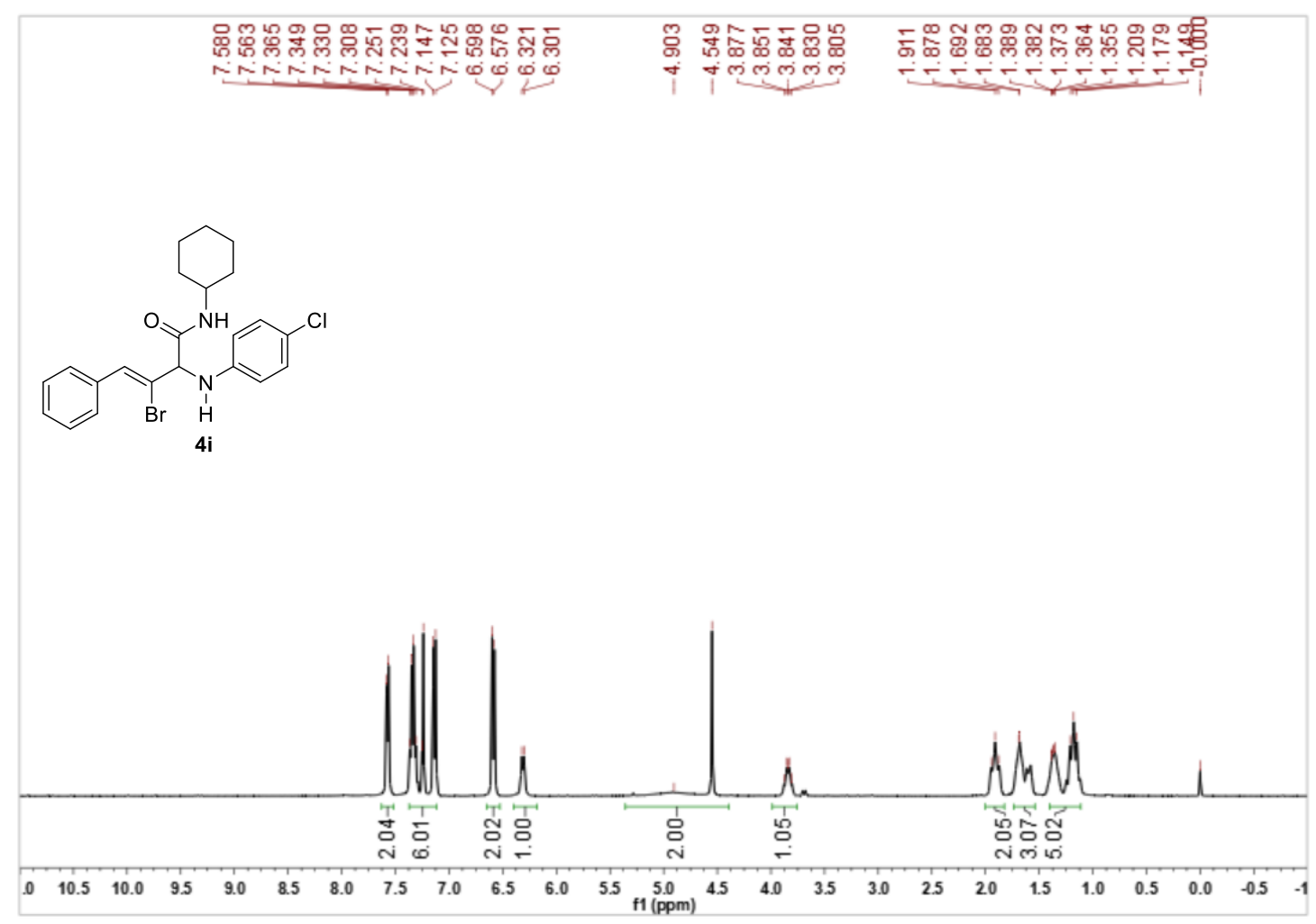

${ }^{1} \mathrm{H}$ NMR (400 MHz), $\mathrm{CDCl}_{3}$

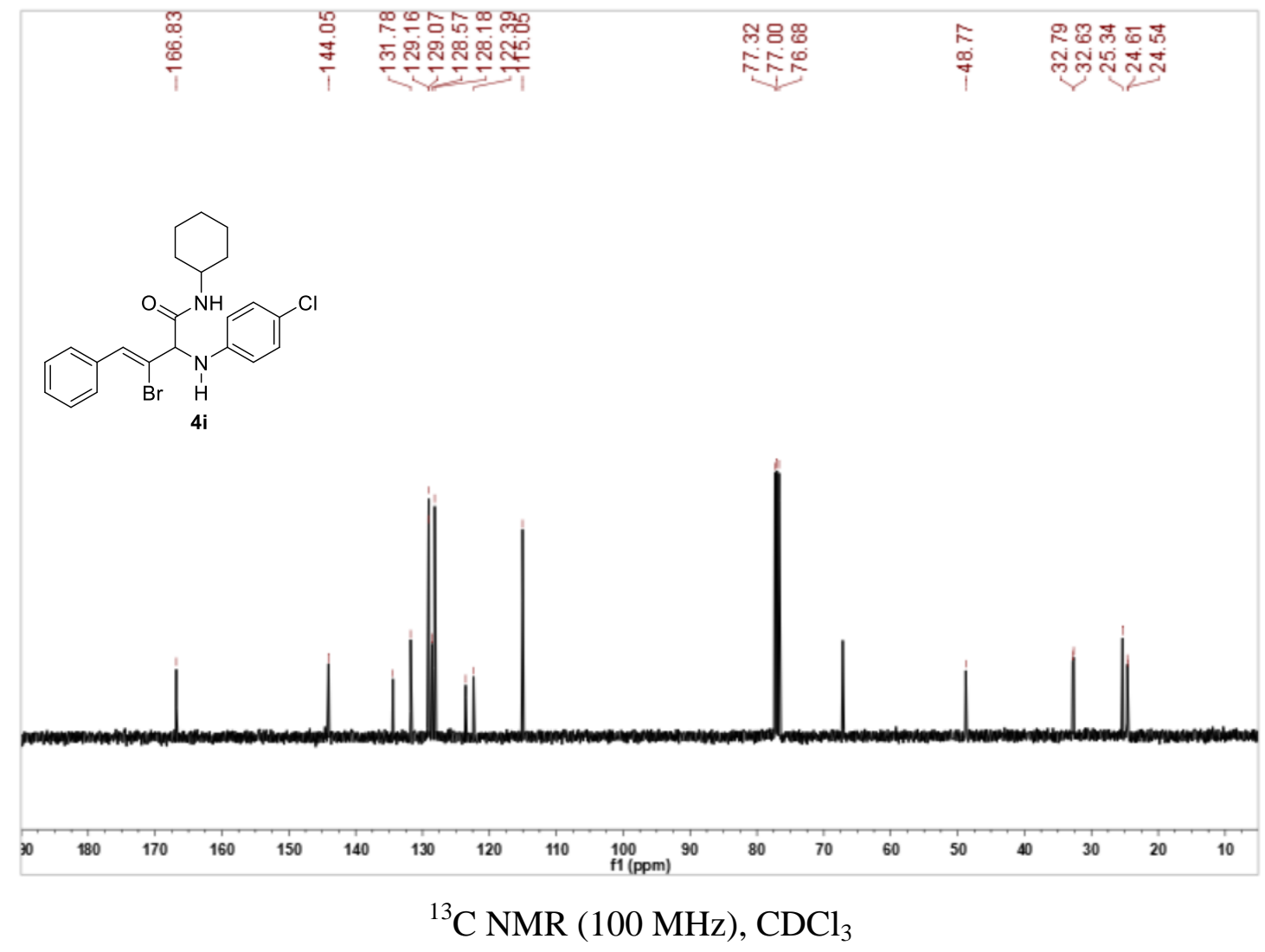




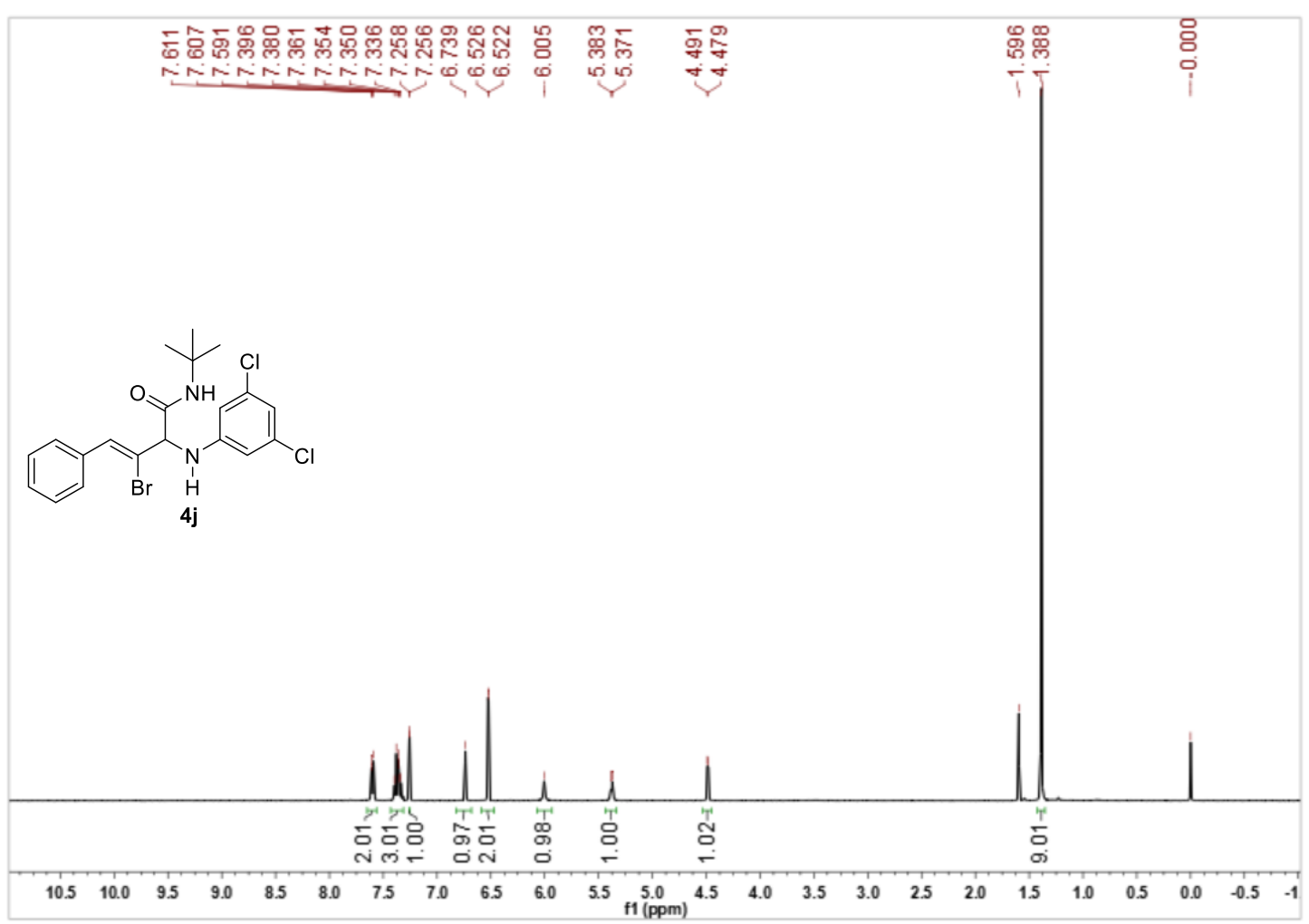

${ }^{1} \mathrm{H}$ NMR (400 MHz), $\mathrm{CDCl}_{3}$

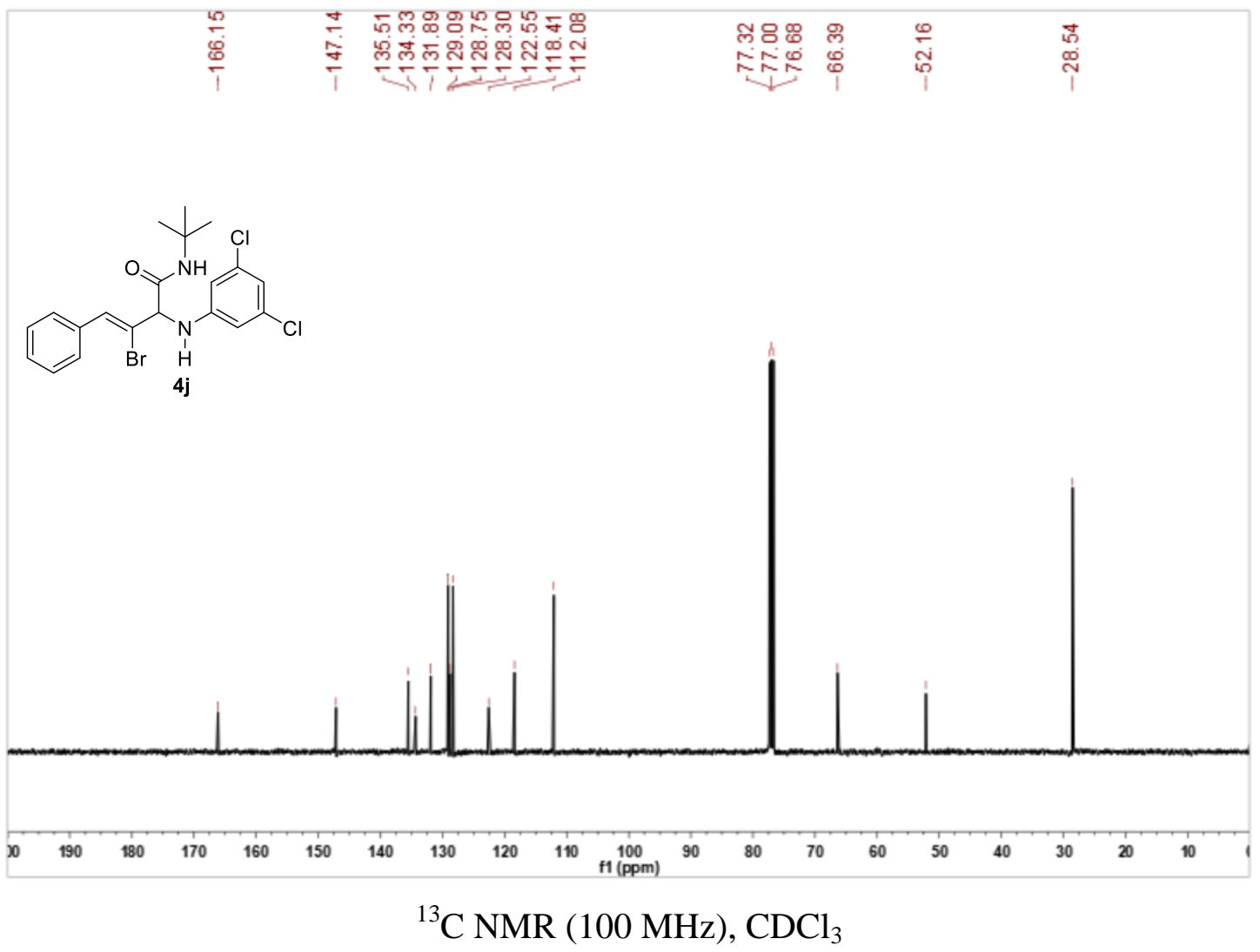




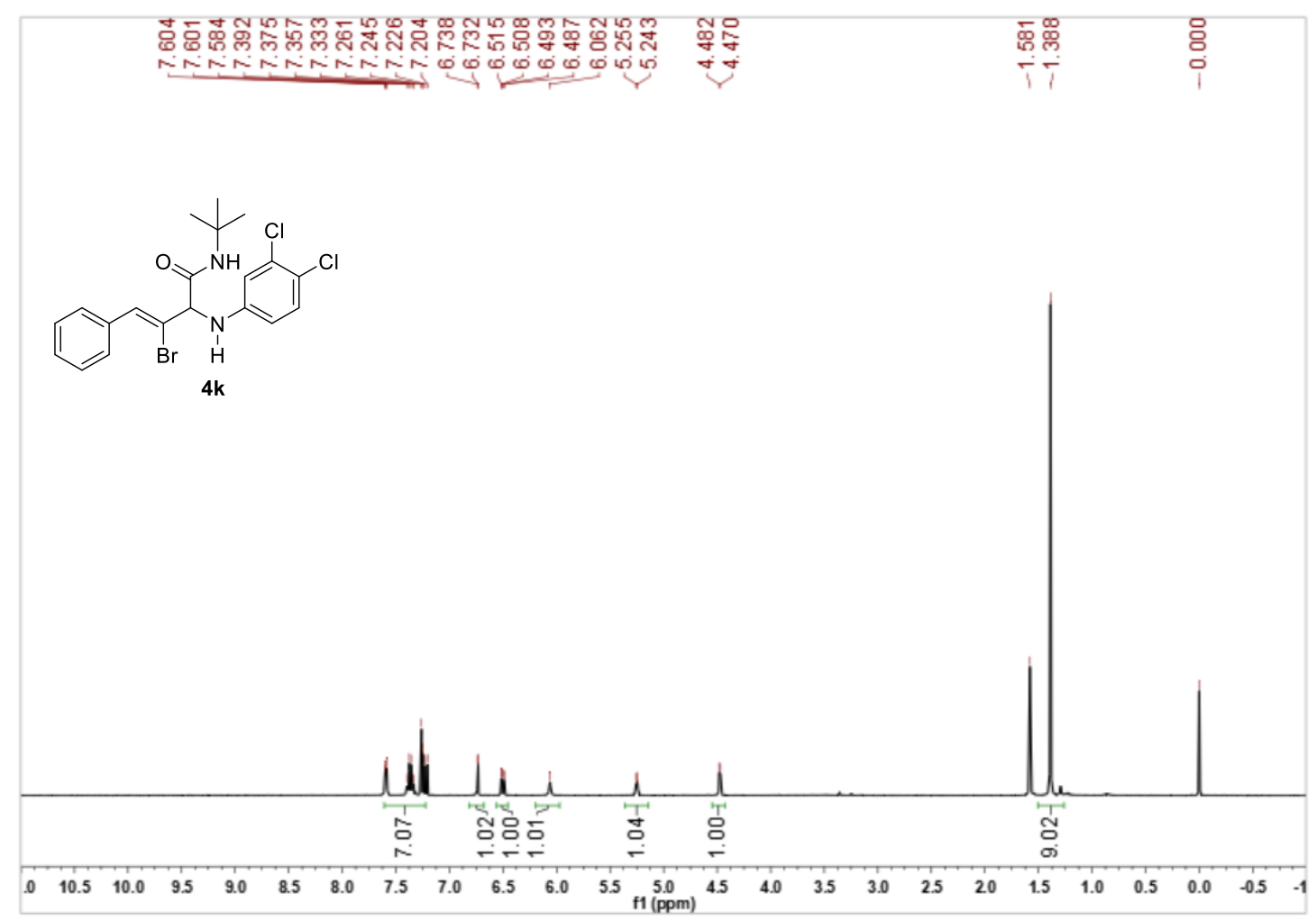

${ }^{1} \mathrm{H}$ NMR (400 MHz), $\mathrm{CDCl}_{3}$

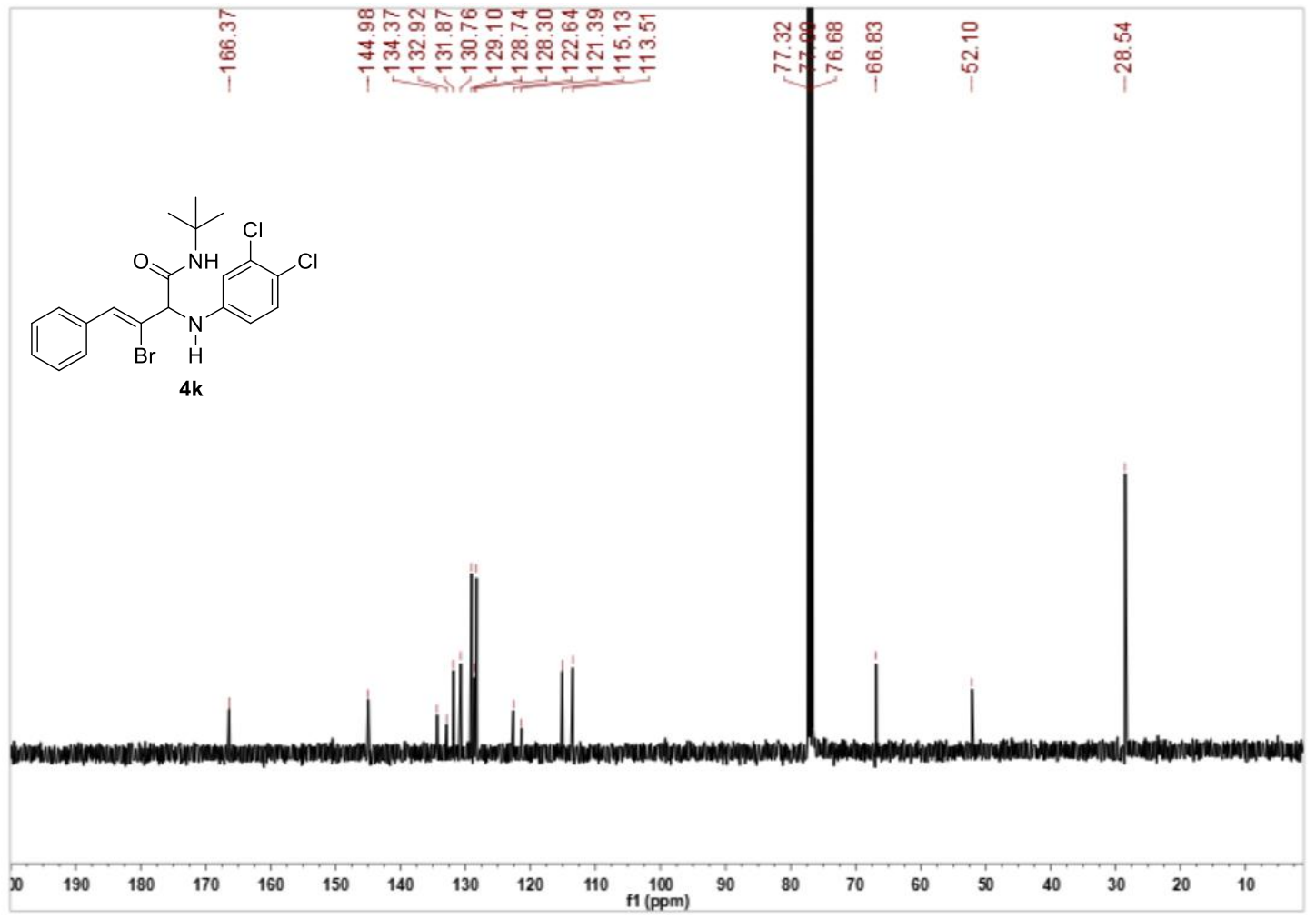

${ }^{13} \mathrm{C}$ NMR (100 MHz), $\mathrm{CDCl}_{3}$ 


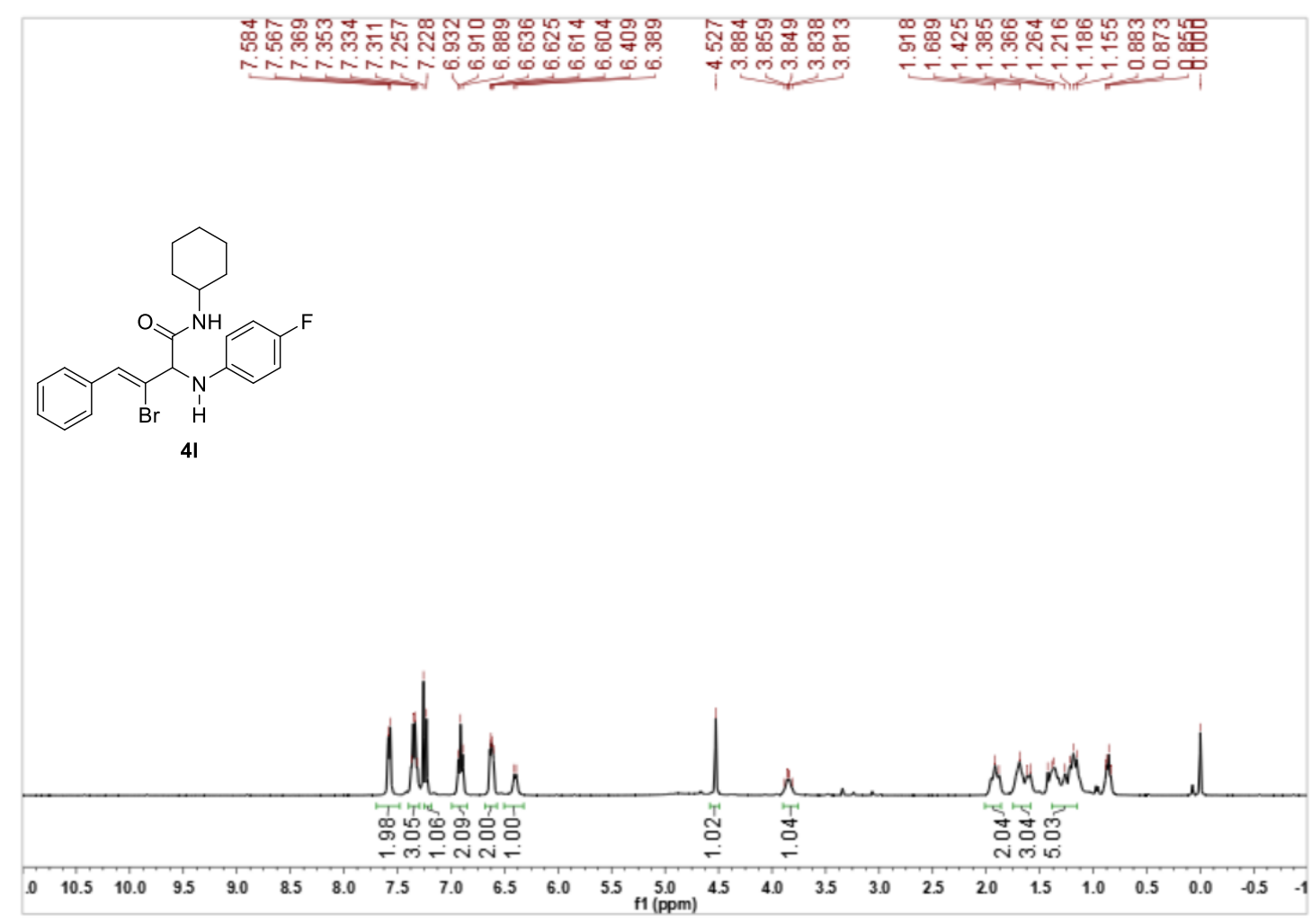

${ }^{1} \mathrm{H}$ NMR (400 MHz), $\mathrm{CDCl}_{3}$

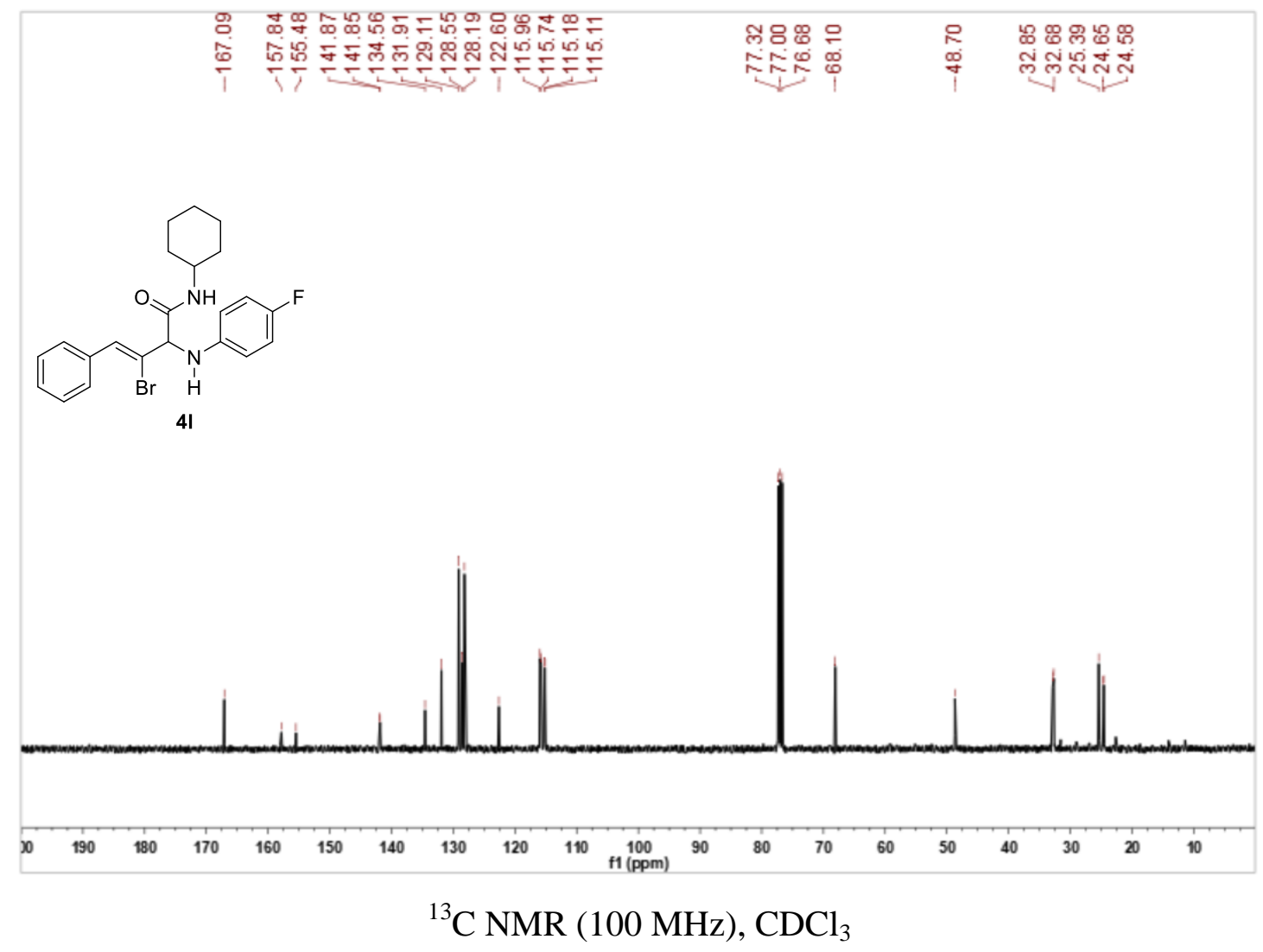




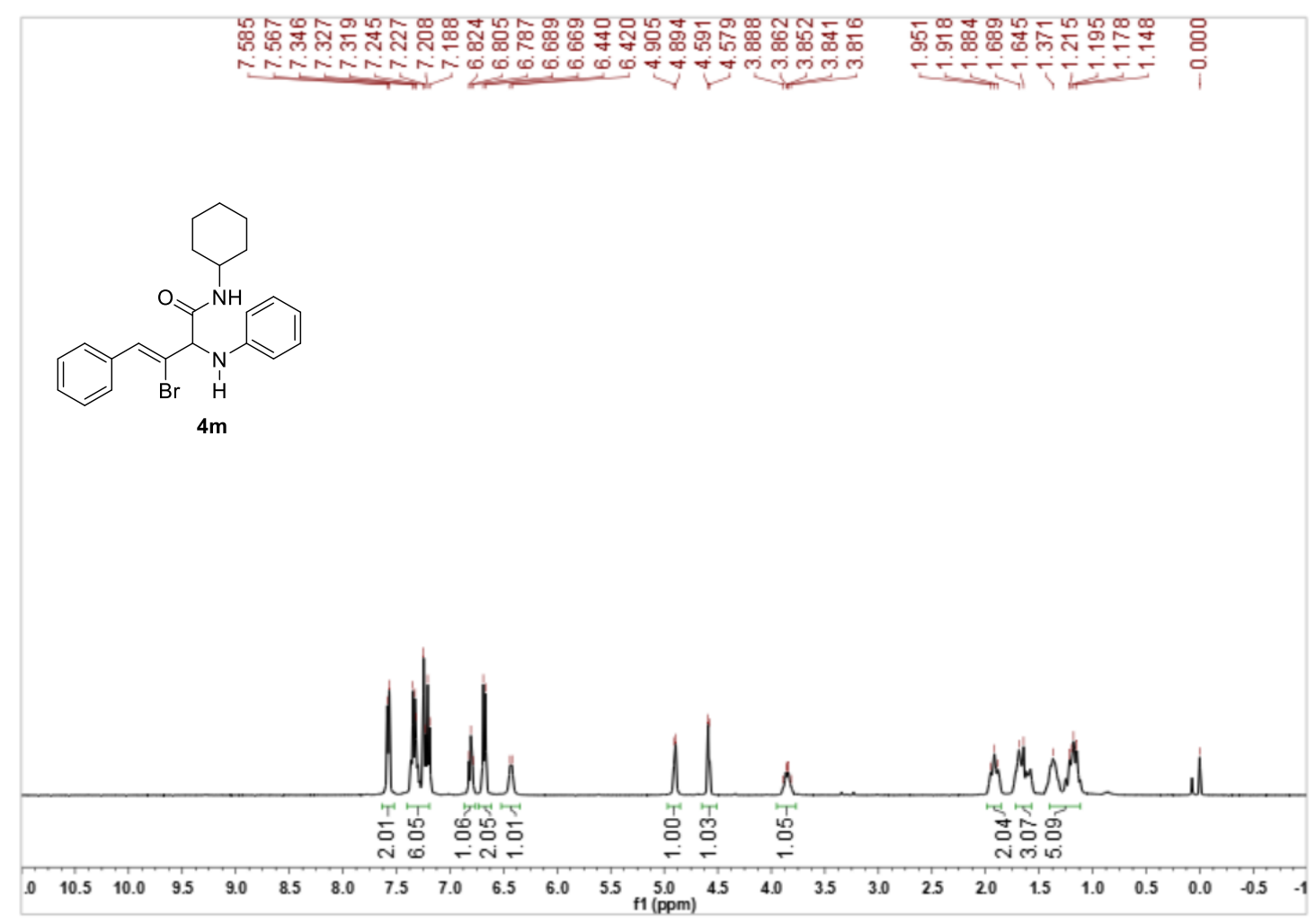

${ }^{1} \mathrm{H}$ NMR (400 MHz), $\mathrm{CDCl}_{3}$

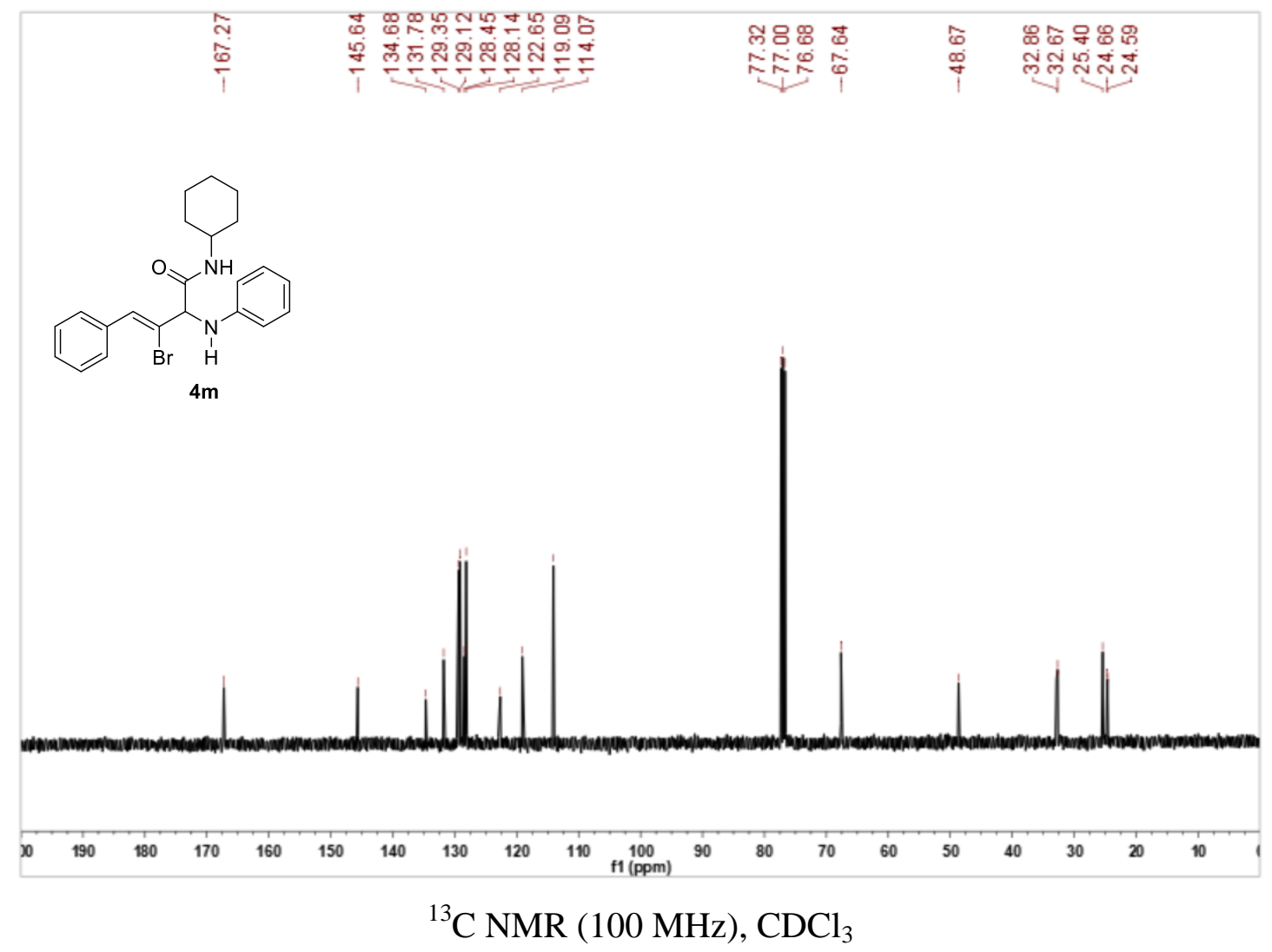




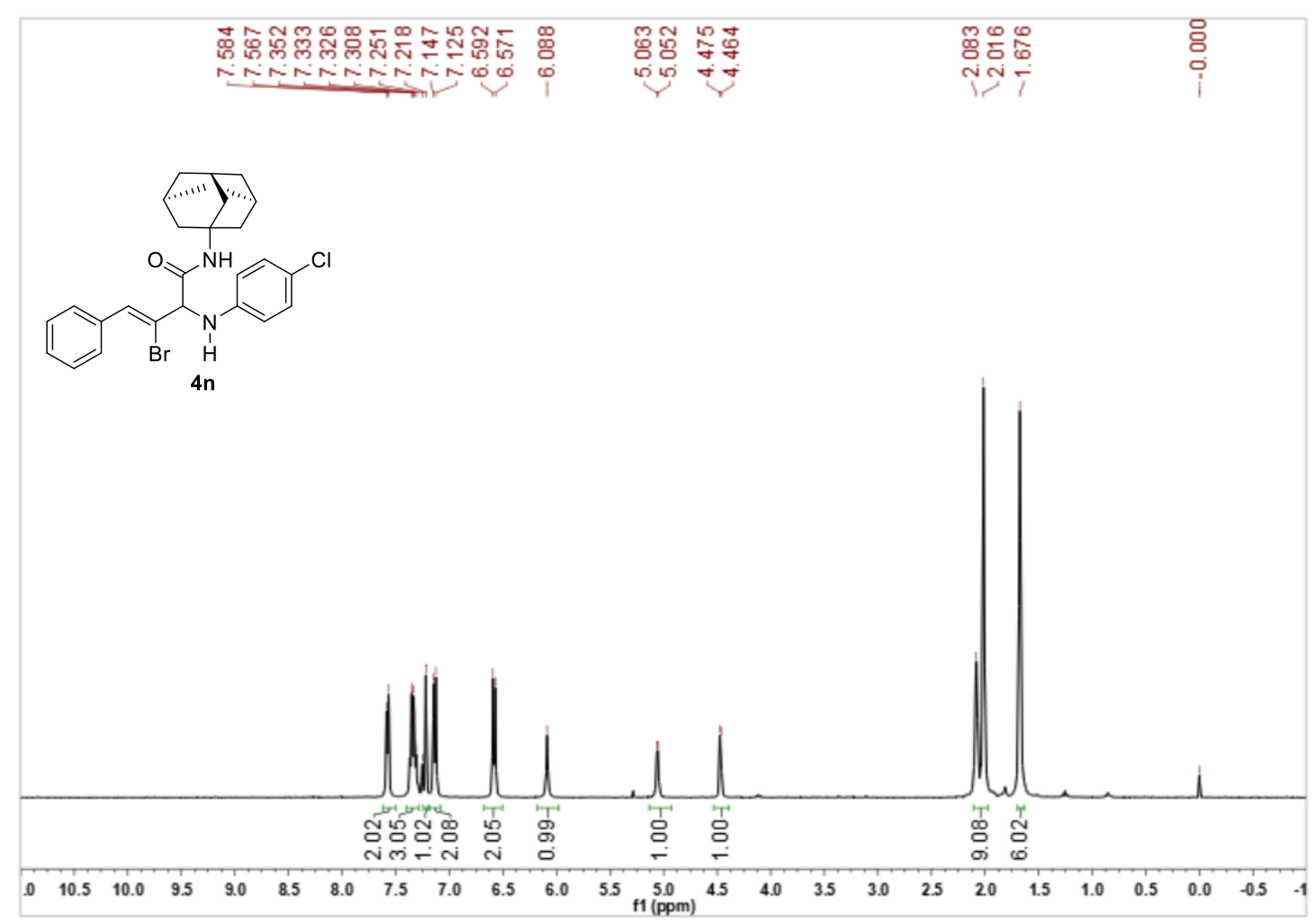

${ }^{1} \mathrm{H}$ NMR (400 MHz), $\mathrm{CDCl}_{3}$

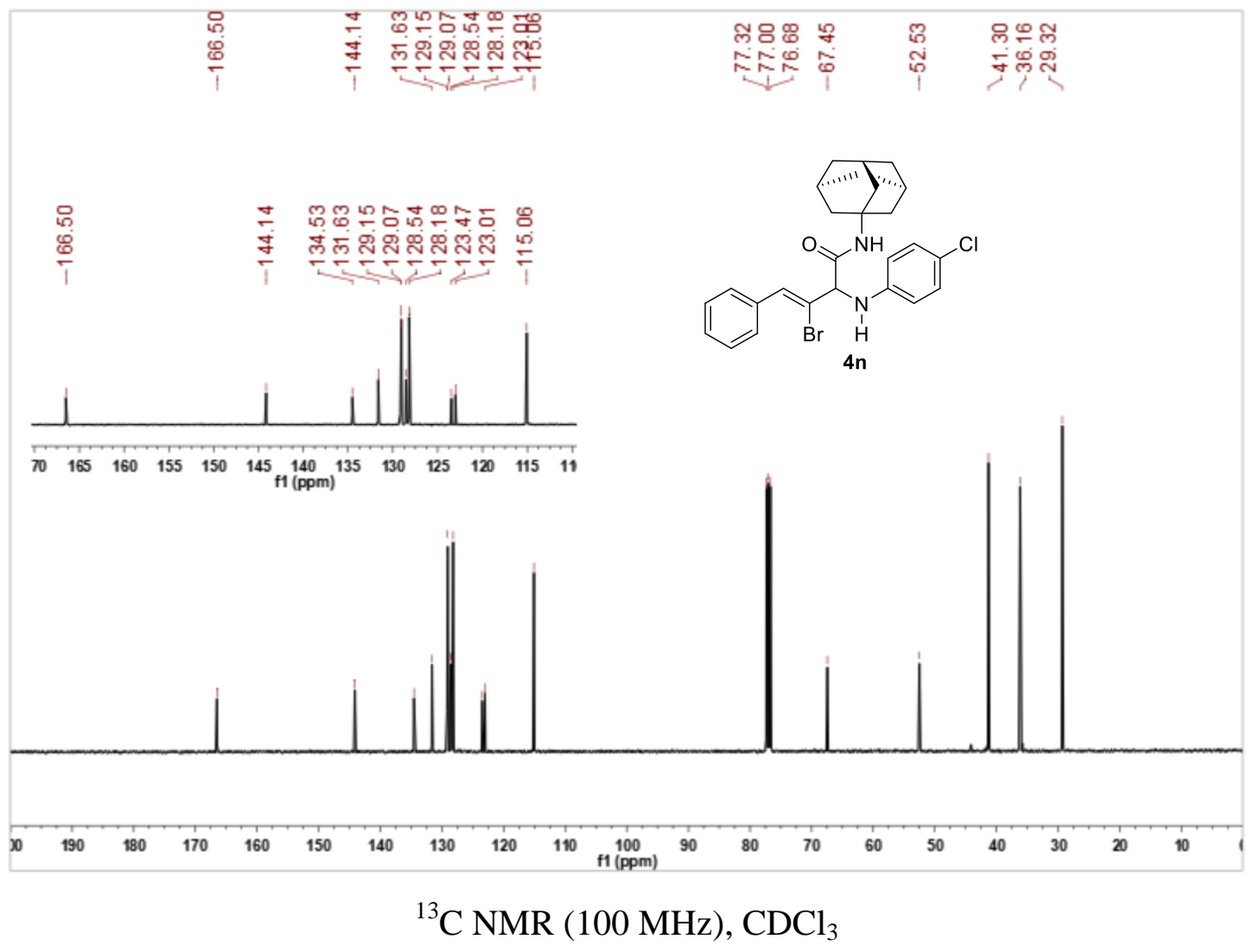




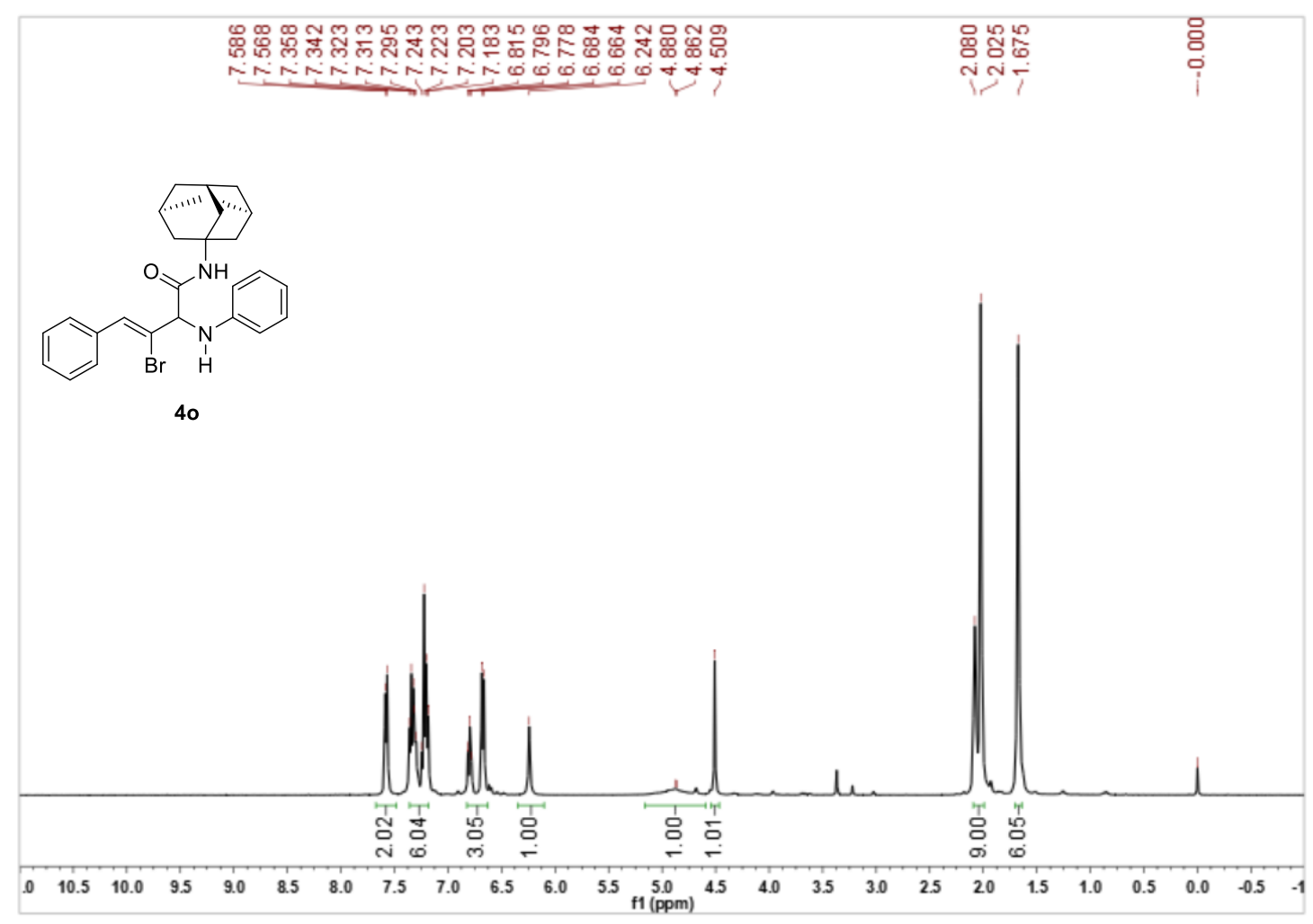

${ }^{1} \mathrm{H}$ NMR (400 MHz), $\mathrm{CDCl}_{3}$

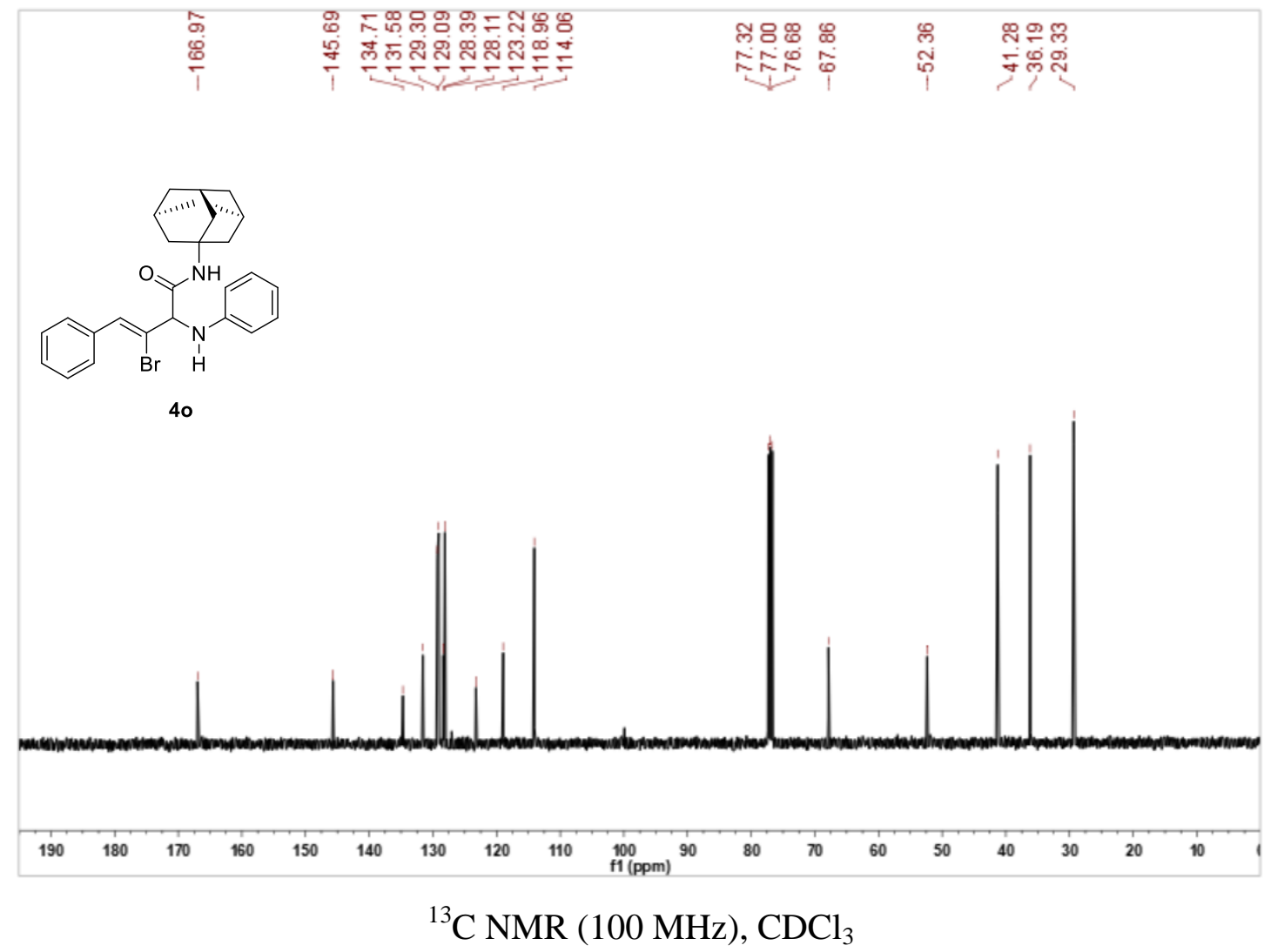




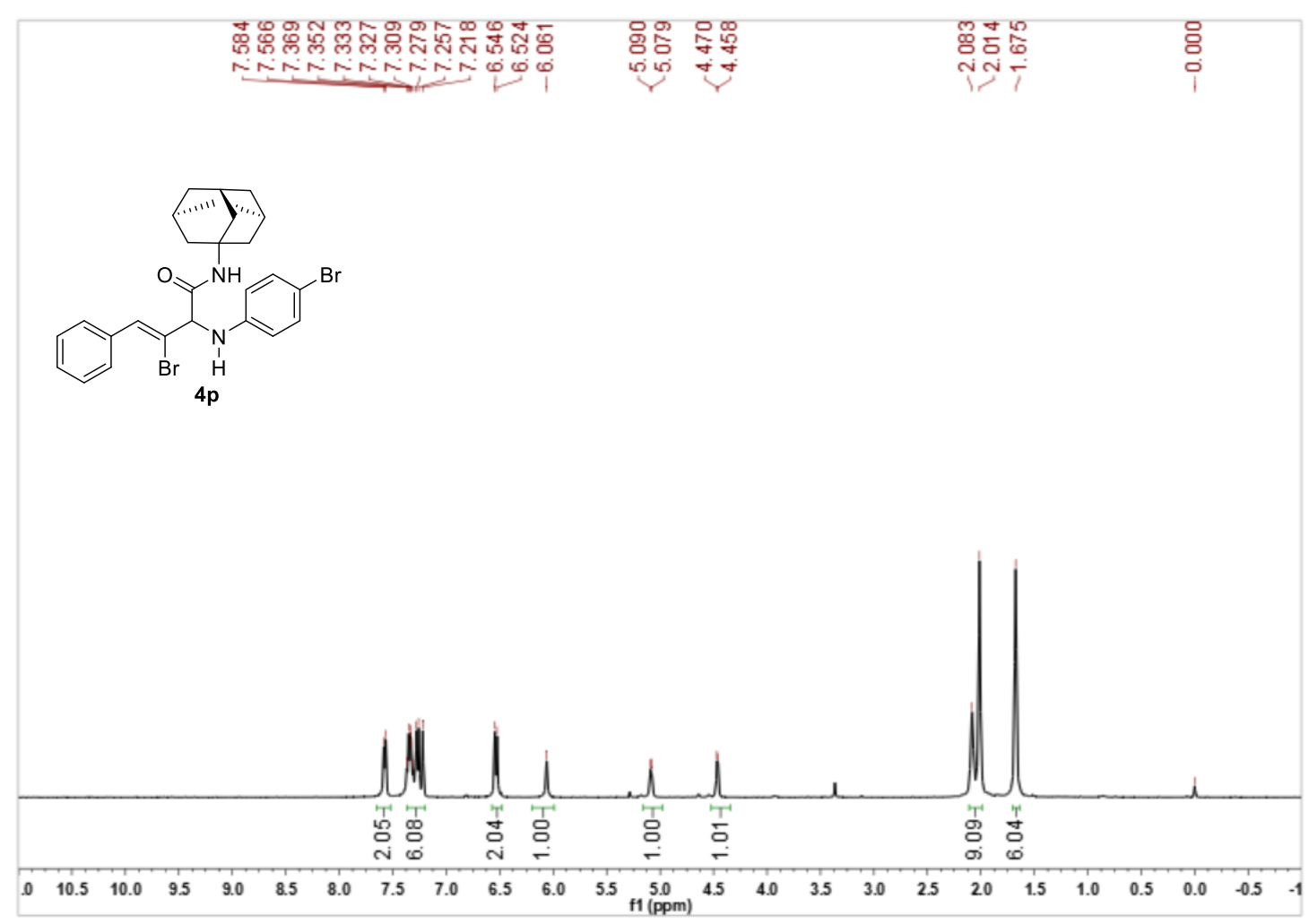

${ }^{1} \mathrm{H}$ NMR (400 MHz), $\mathrm{CDCl}_{3}$

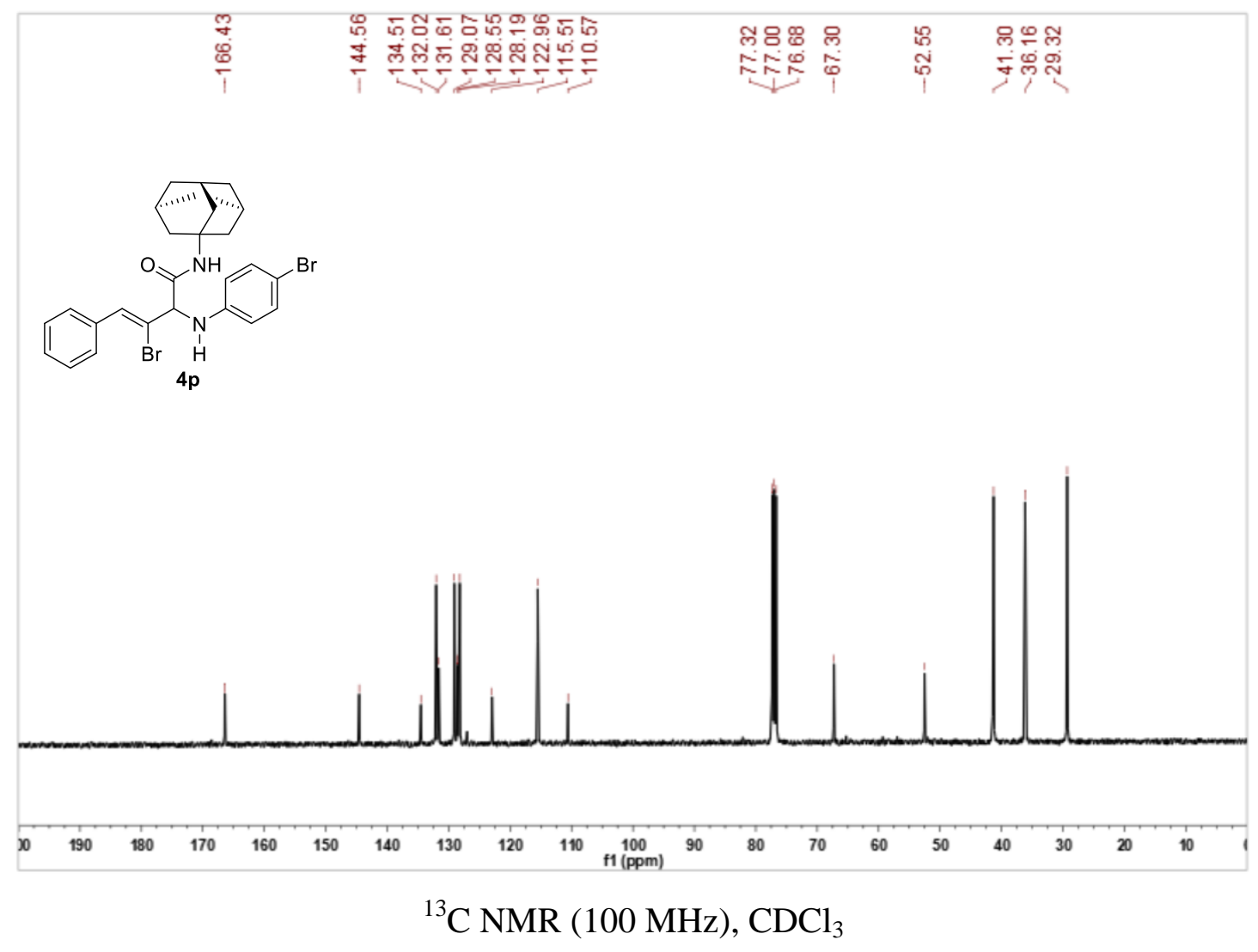




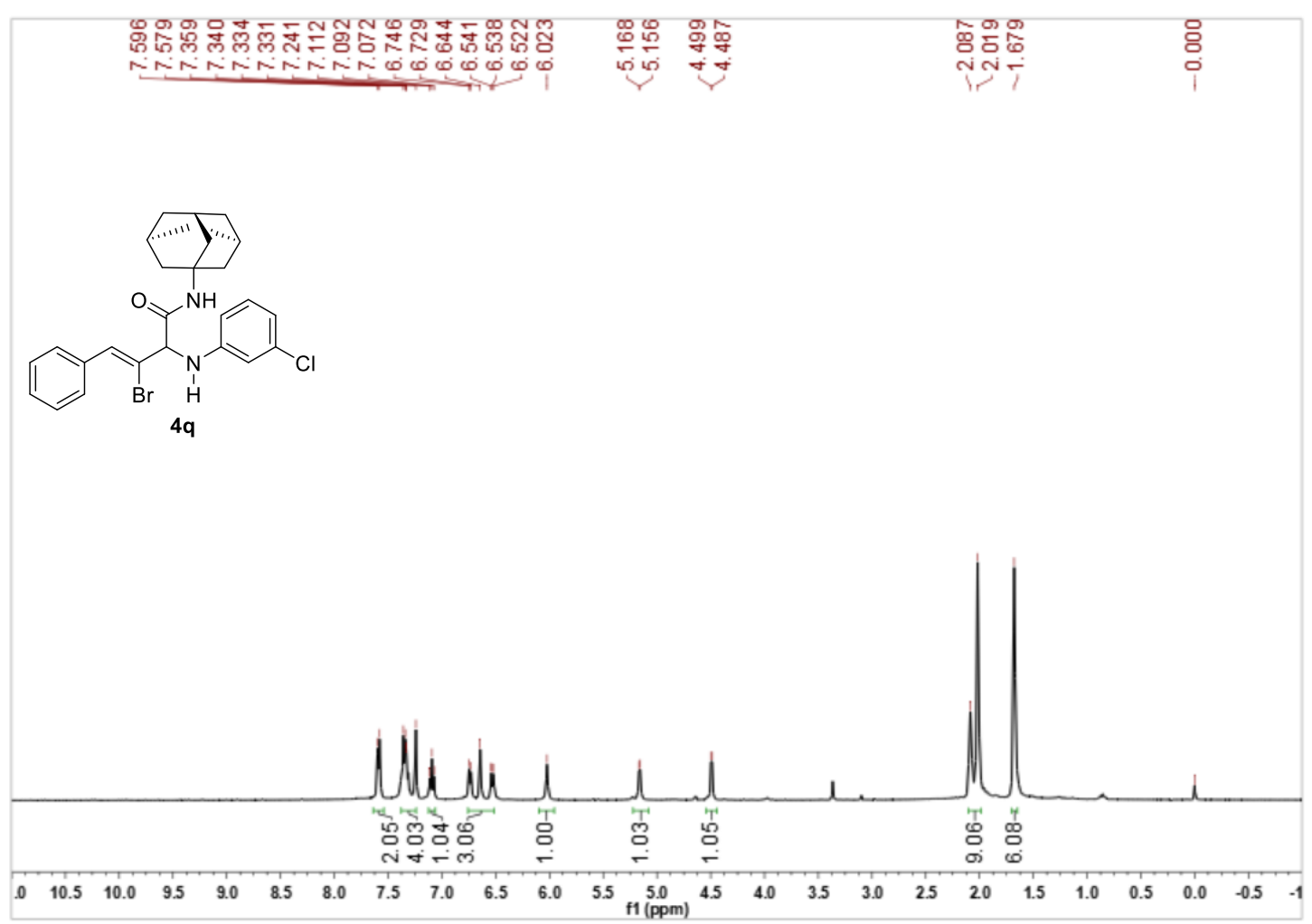

${ }^{1} \mathrm{H}$ NMR (400 MHz), $\mathrm{CDCl}_{3}$

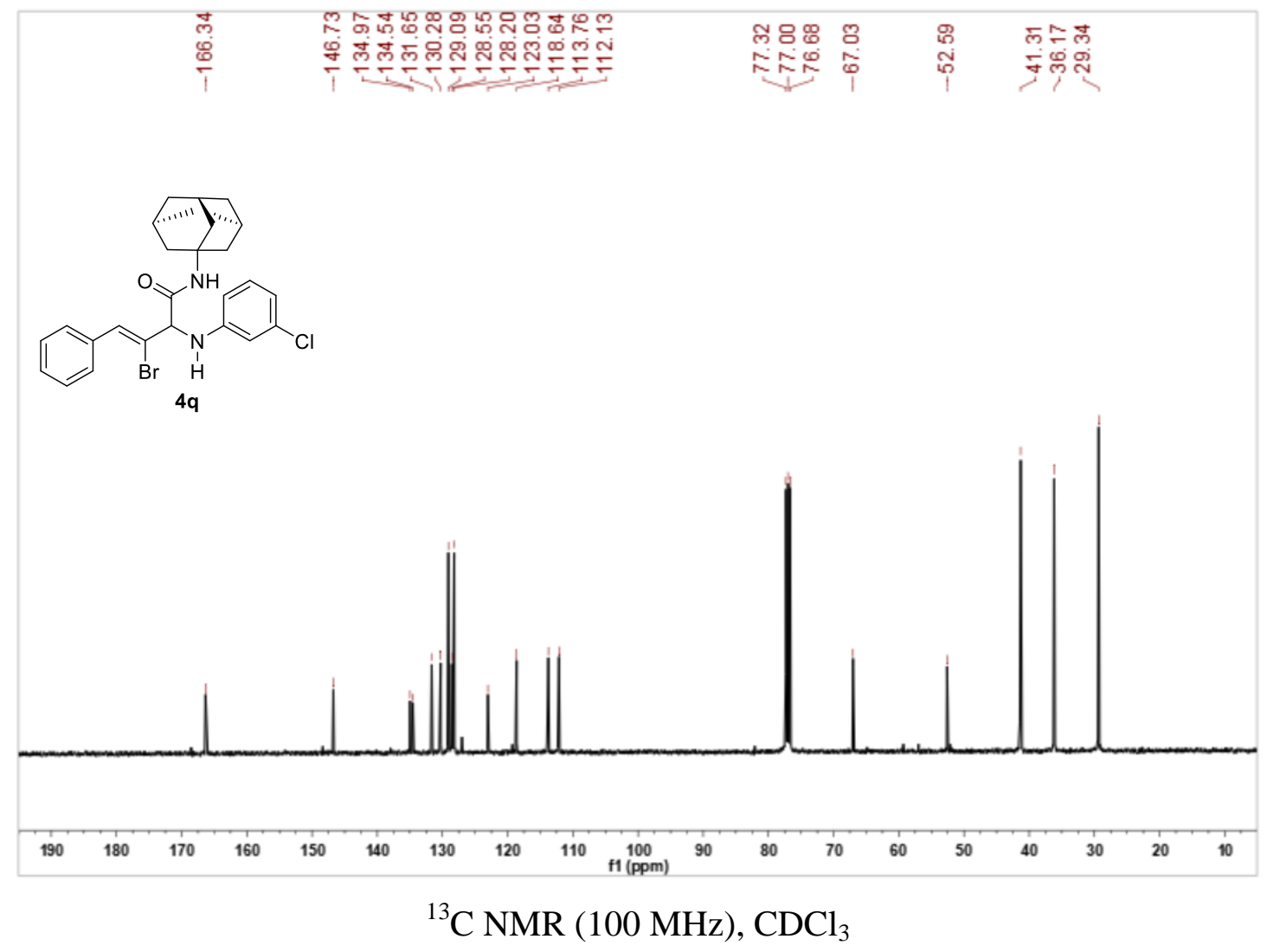




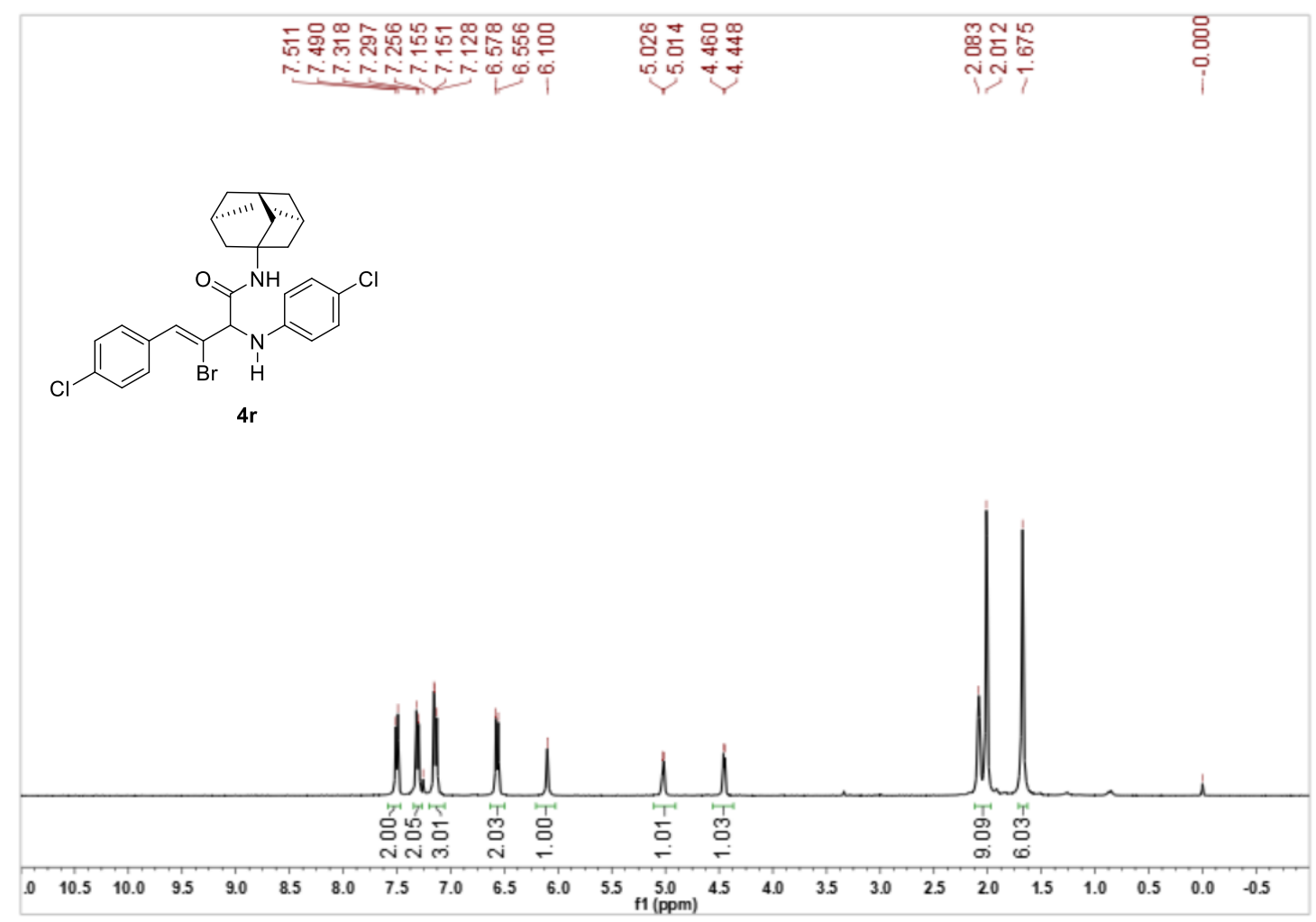

${ }^{1} \mathrm{H}$ NMR (400 MHz), $\mathrm{CDCl}_{3}$

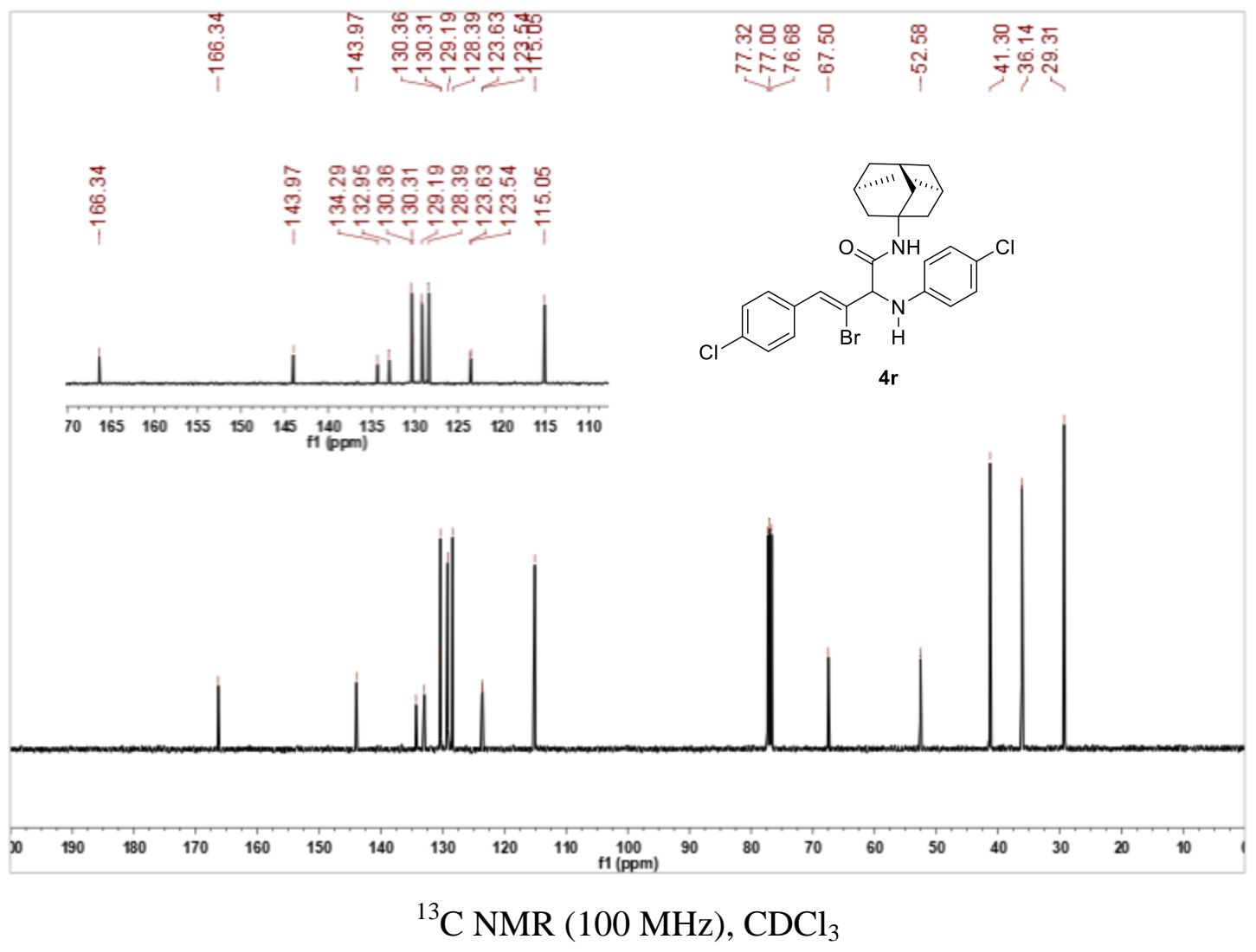




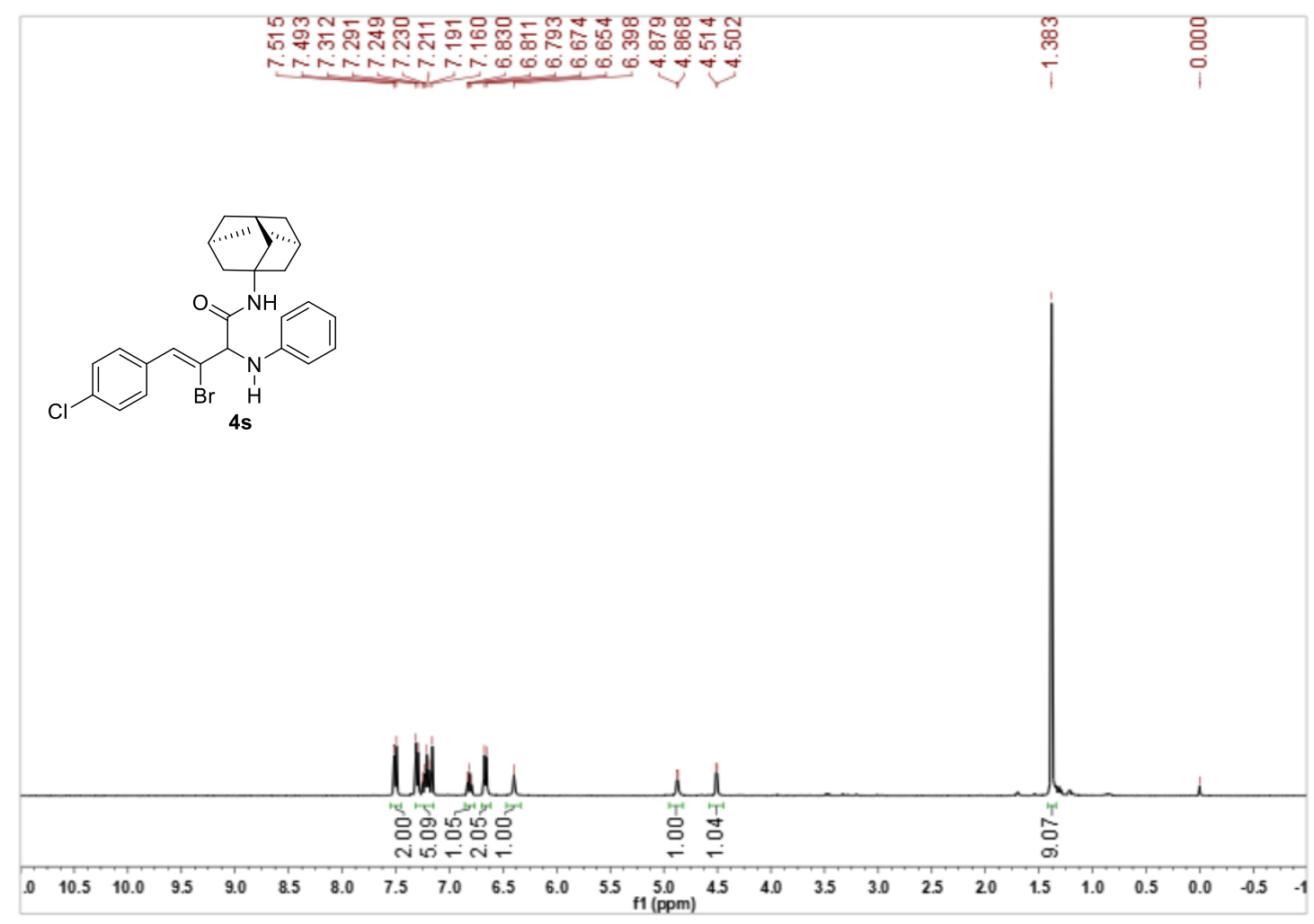

${ }^{1} \mathrm{H}$ NMR (400 MHz), $\mathrm{CDCl}_{3}$

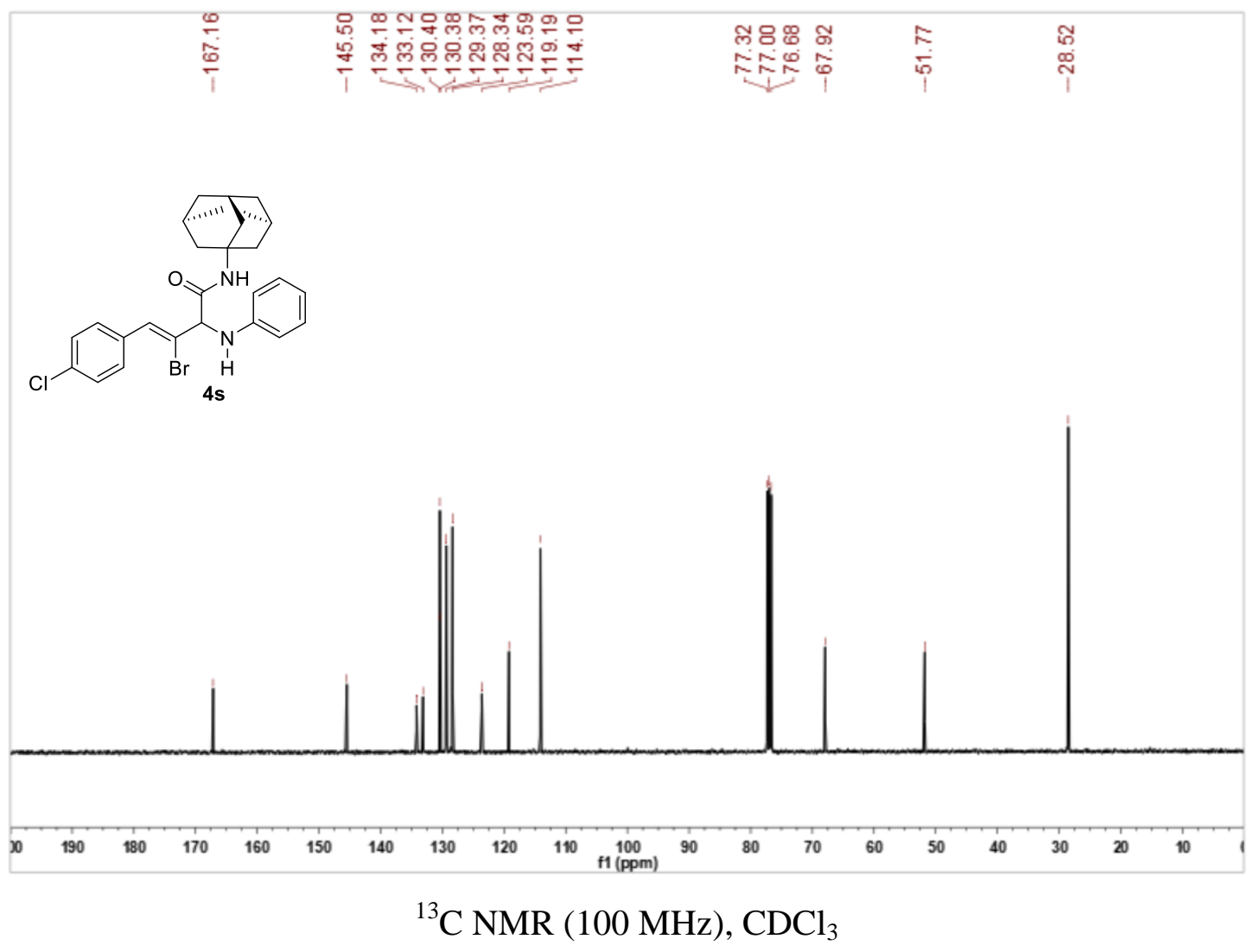




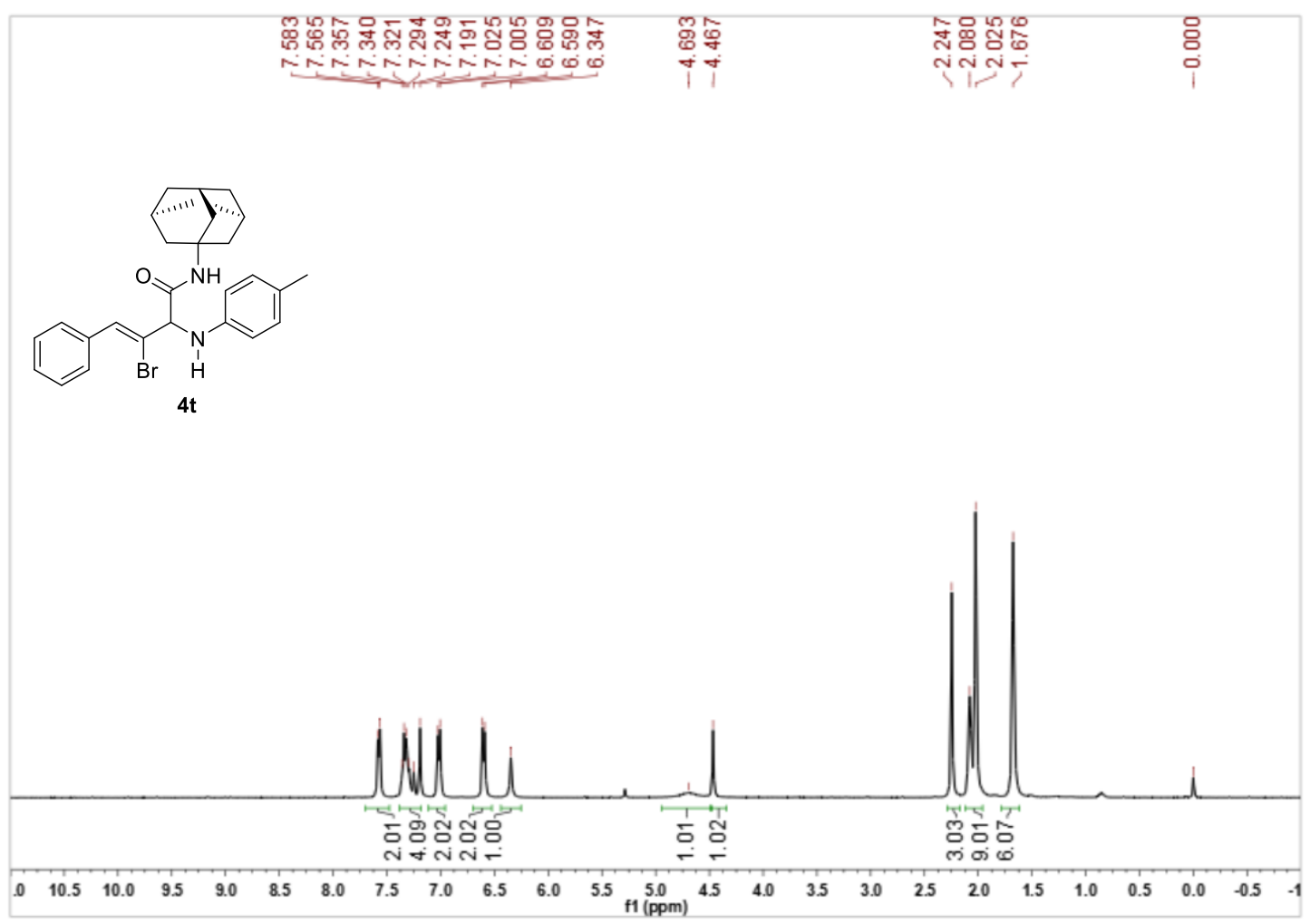

${ }^{1} \mathrm{H}$ NMR (400 MHz), $\mathrm{CDCl}_{3}$

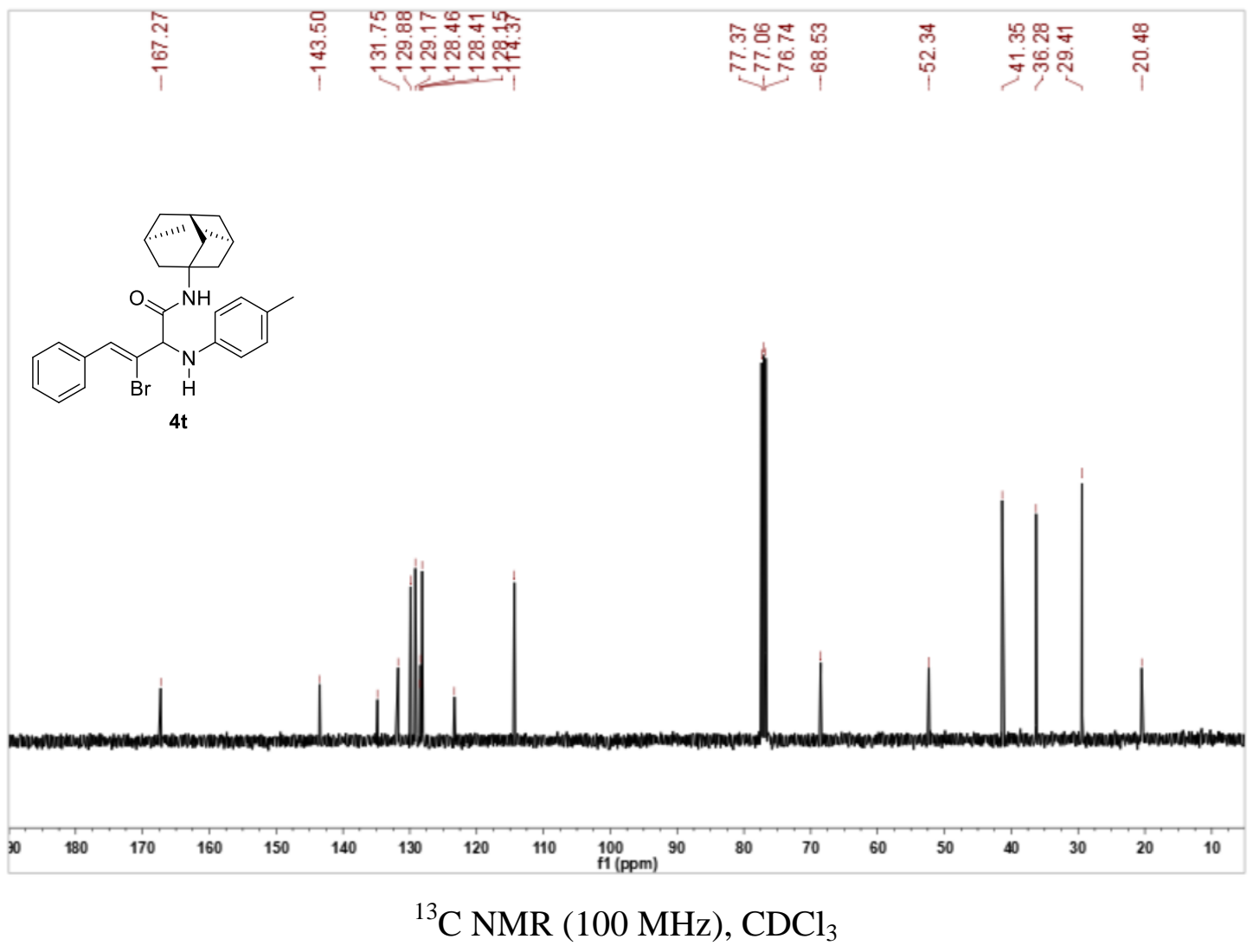




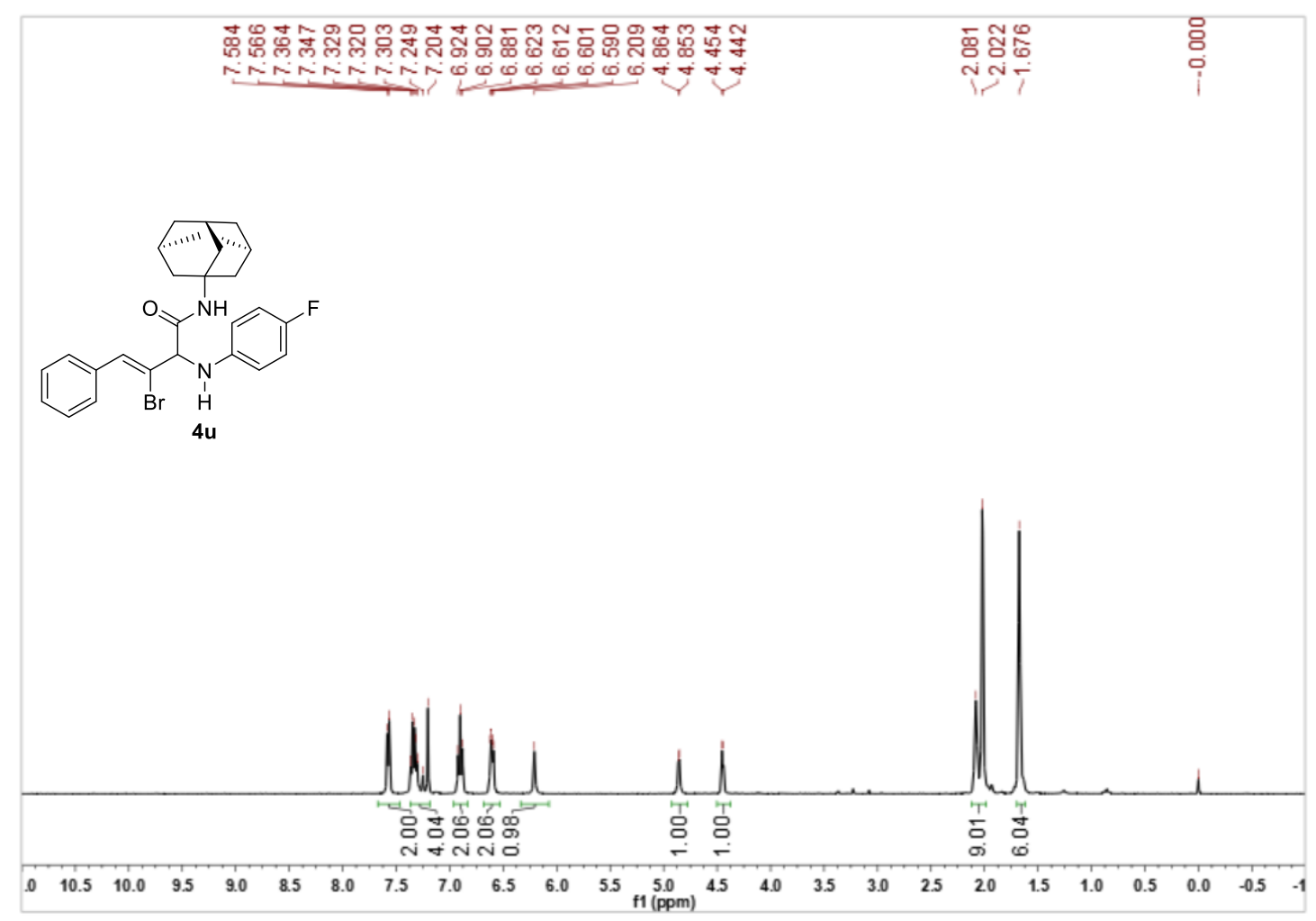

${ }^{1} \mathrm{H}$ NMR (400 MHz), $\mathrm{CDCl}_{3}$

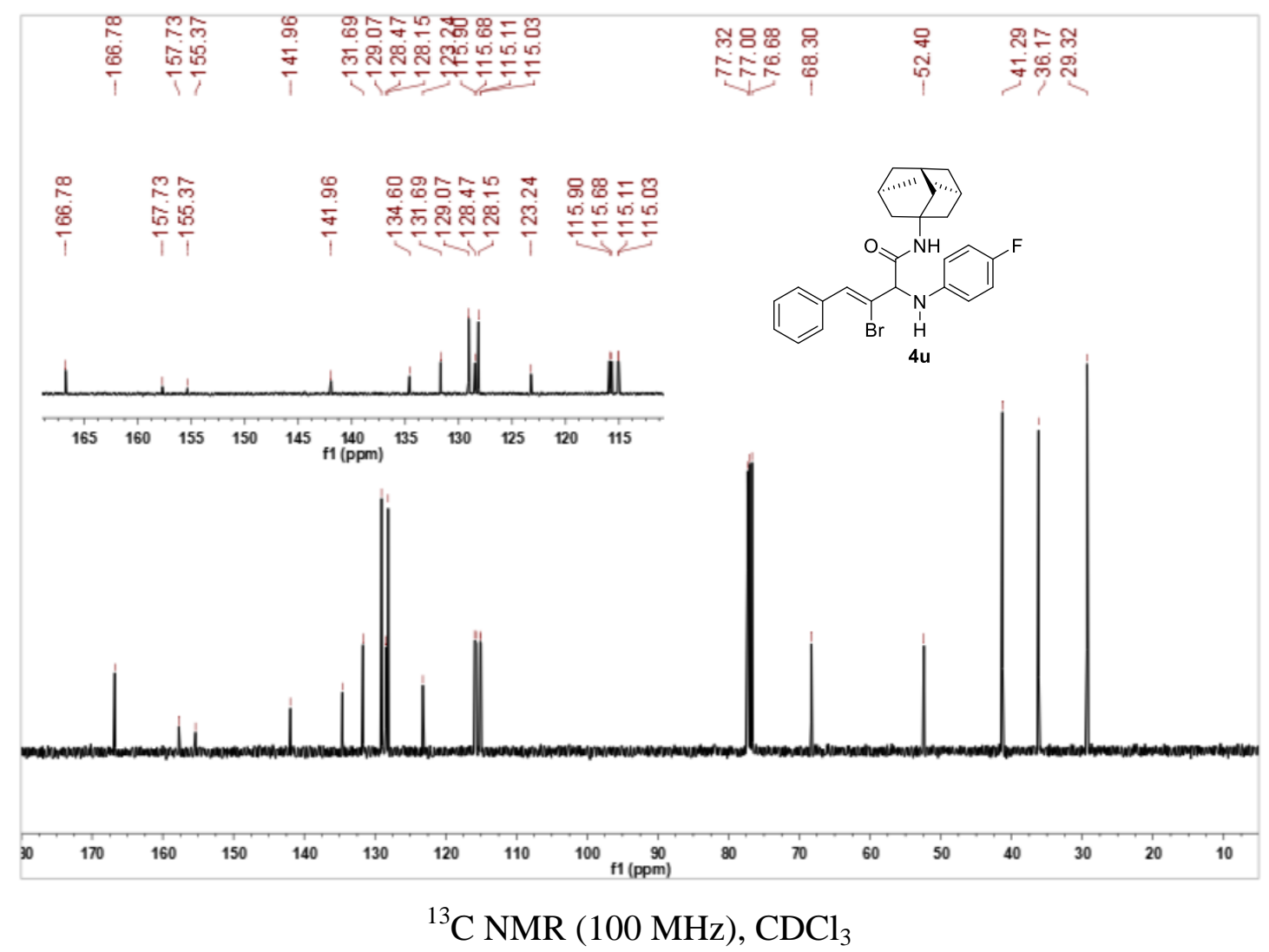




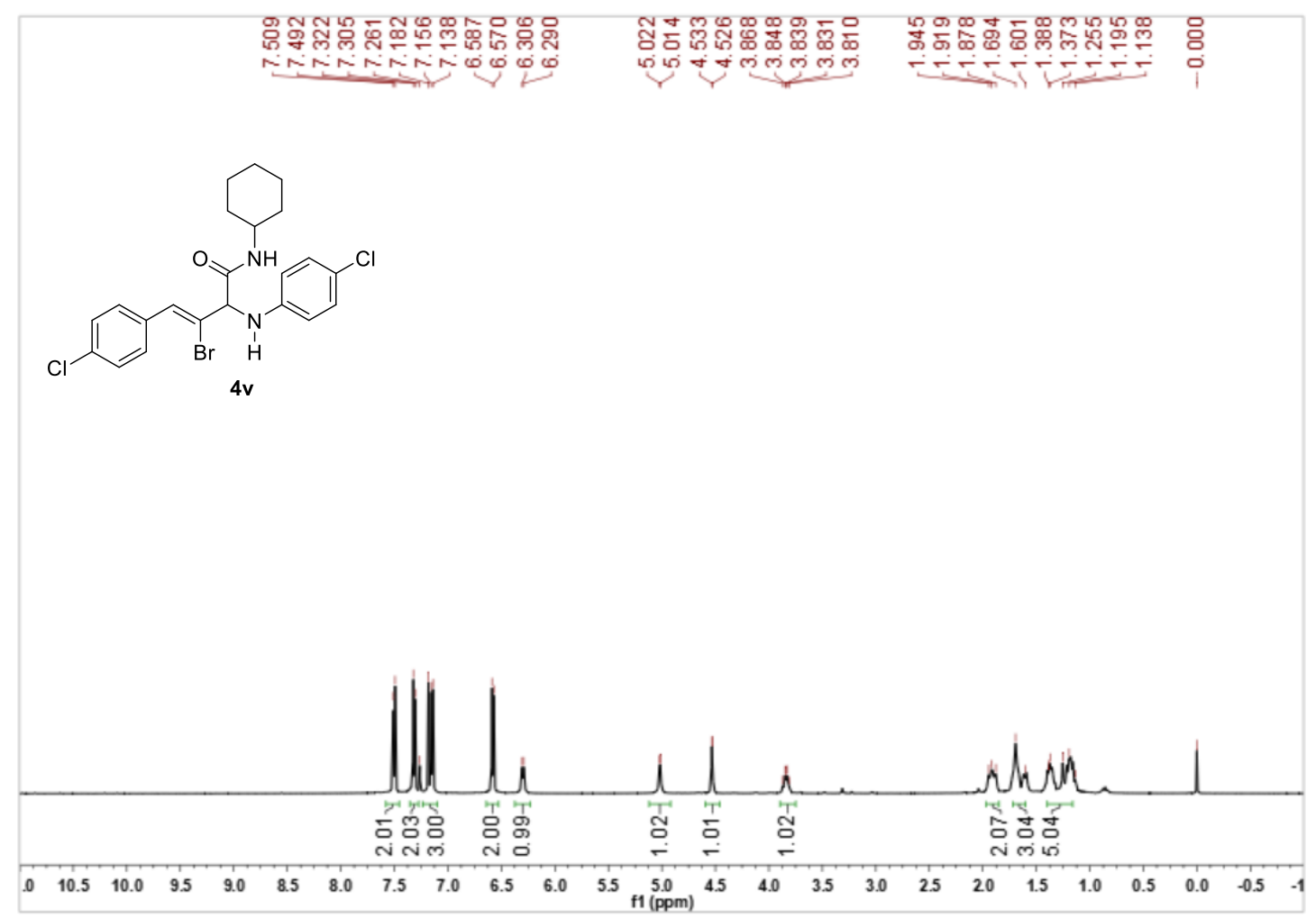

${ }^{1} \mathrm{H}$ NMR $(400 \mathrm{MHz}), \mathrm{CDCl}_{3}$

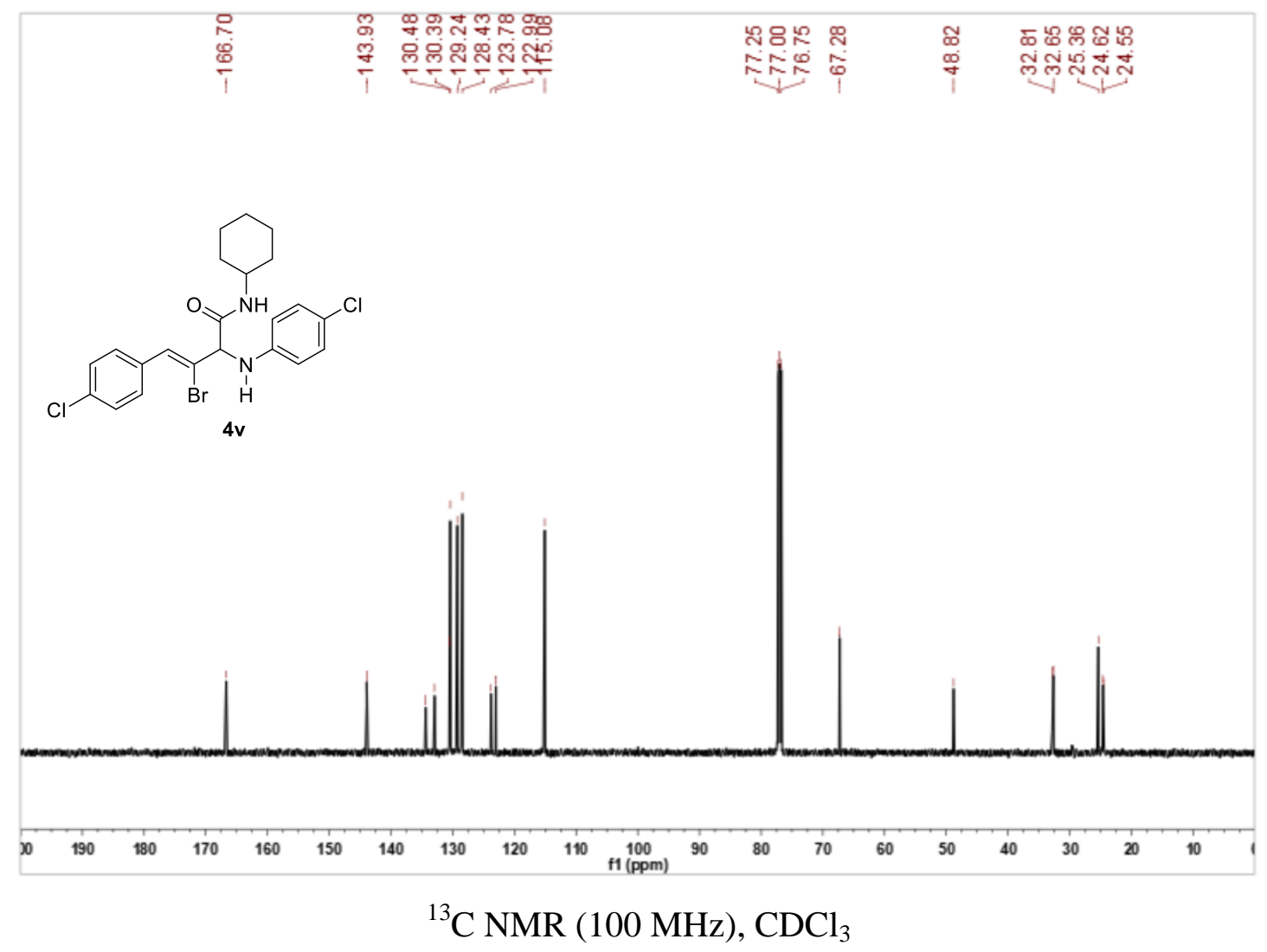




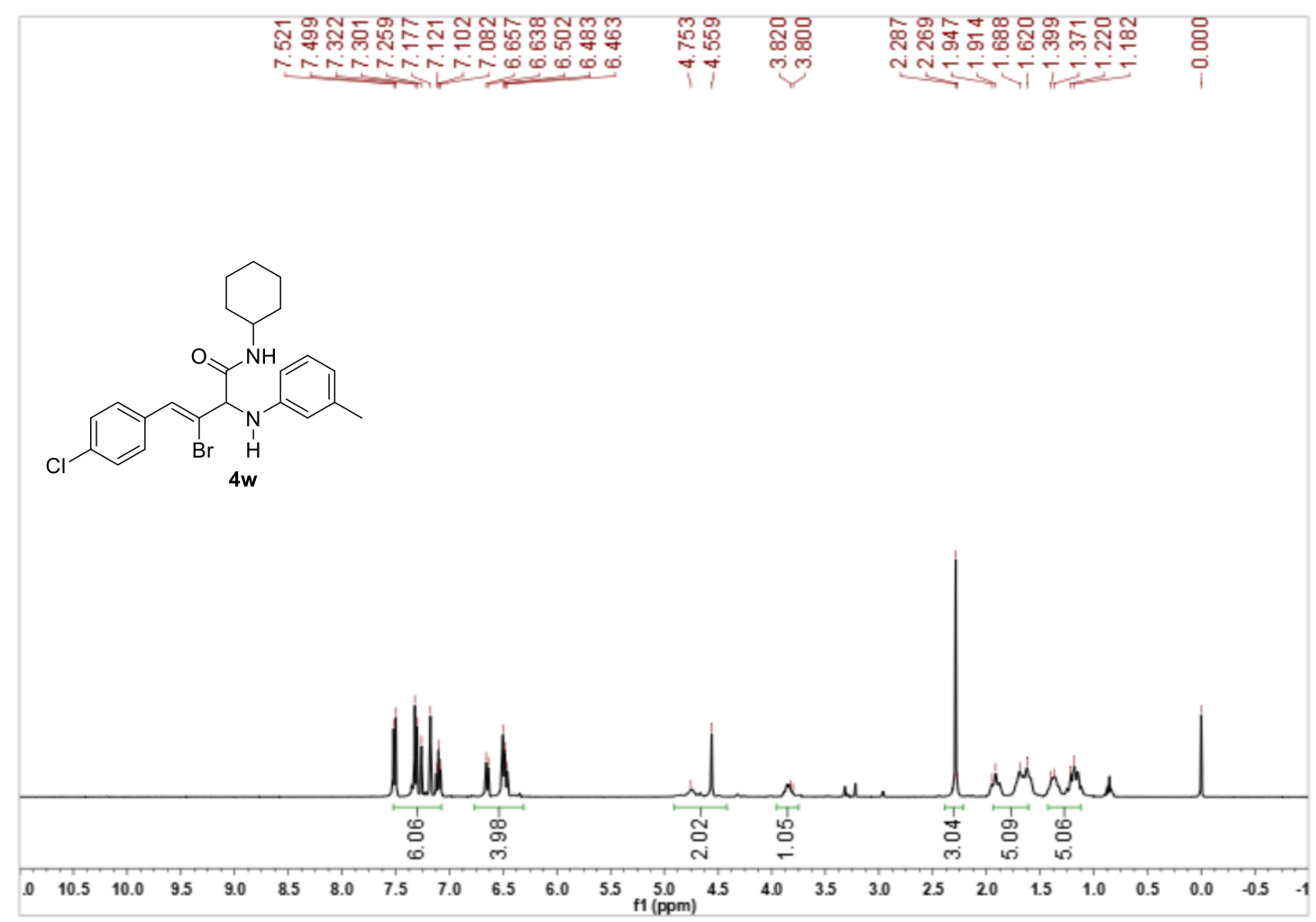

${ }^{1} \mathrm{H}$ NMR (400 MHz), $\mathrm{CDCl}_{3}$

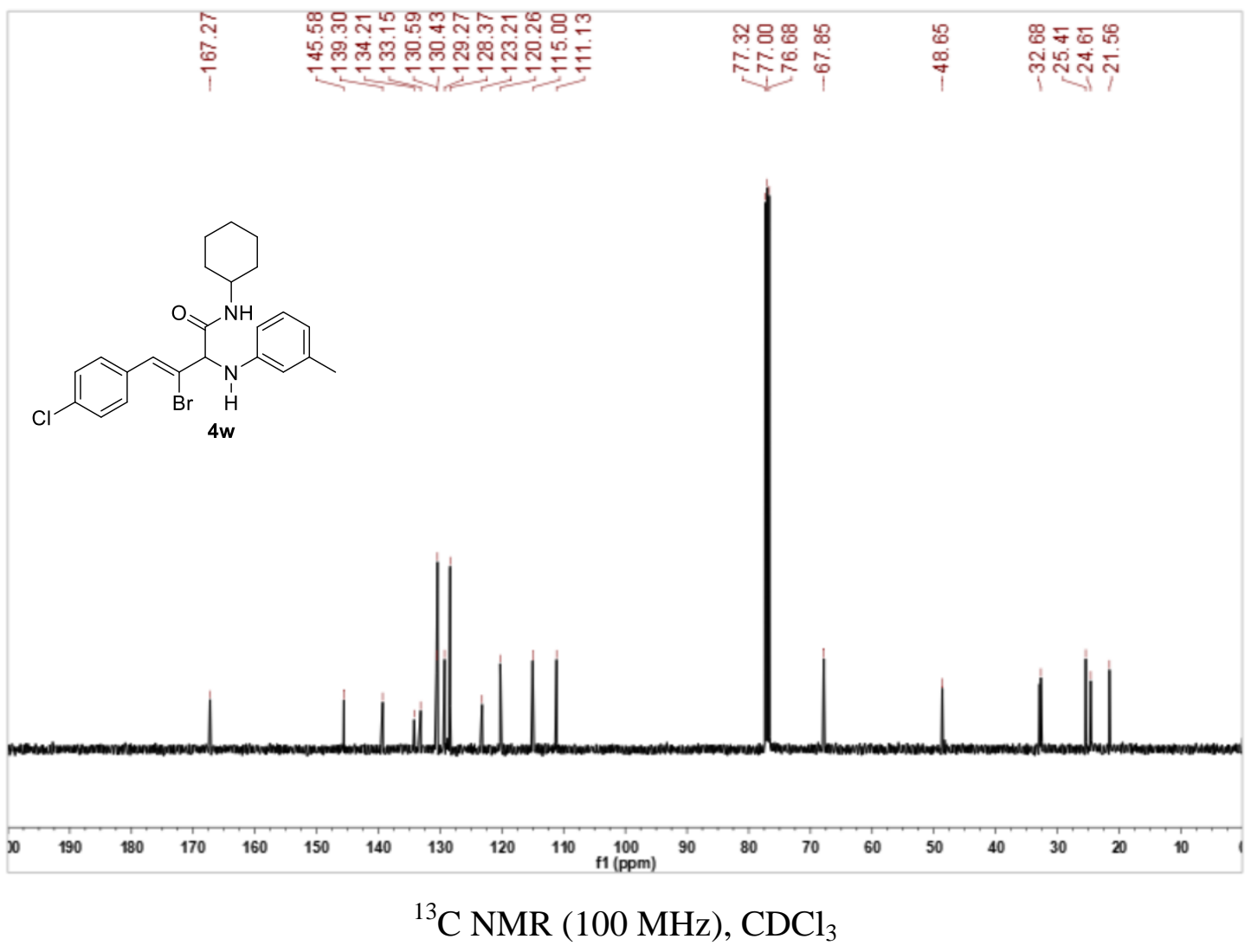




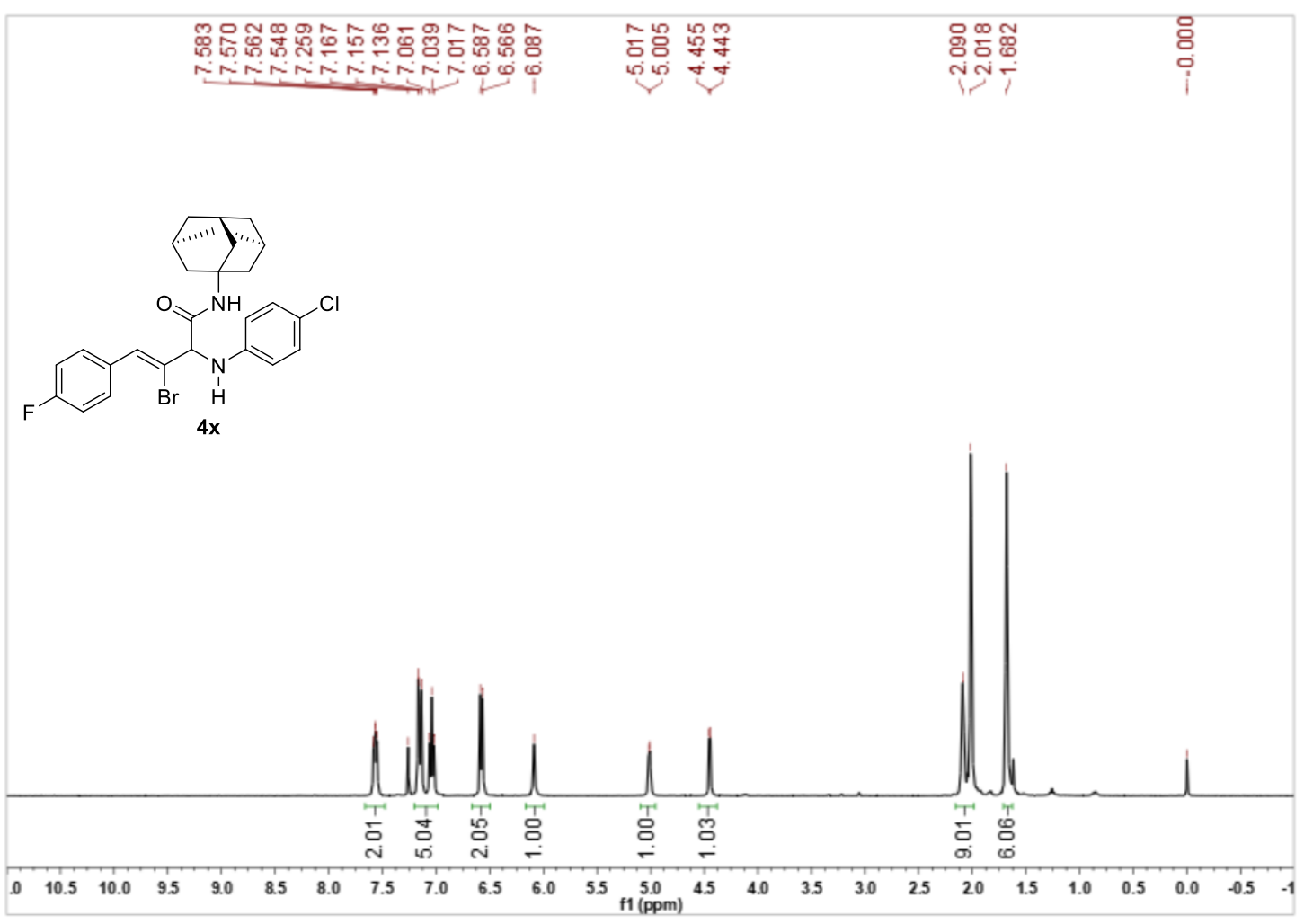

${ }^{1} \mathrm{H}$ NMR (400 MHz), $\mathrm{CDCl}_{3}$

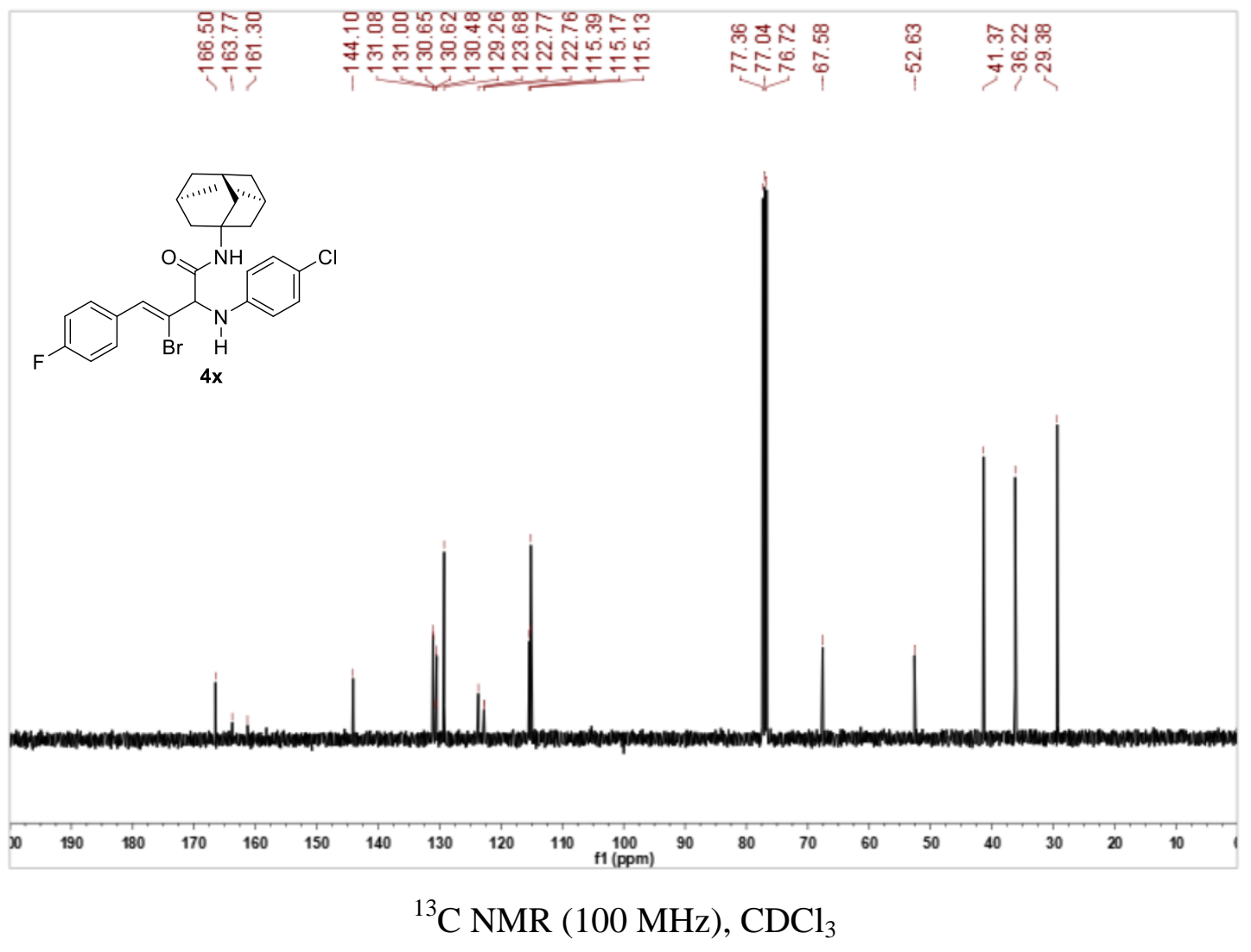




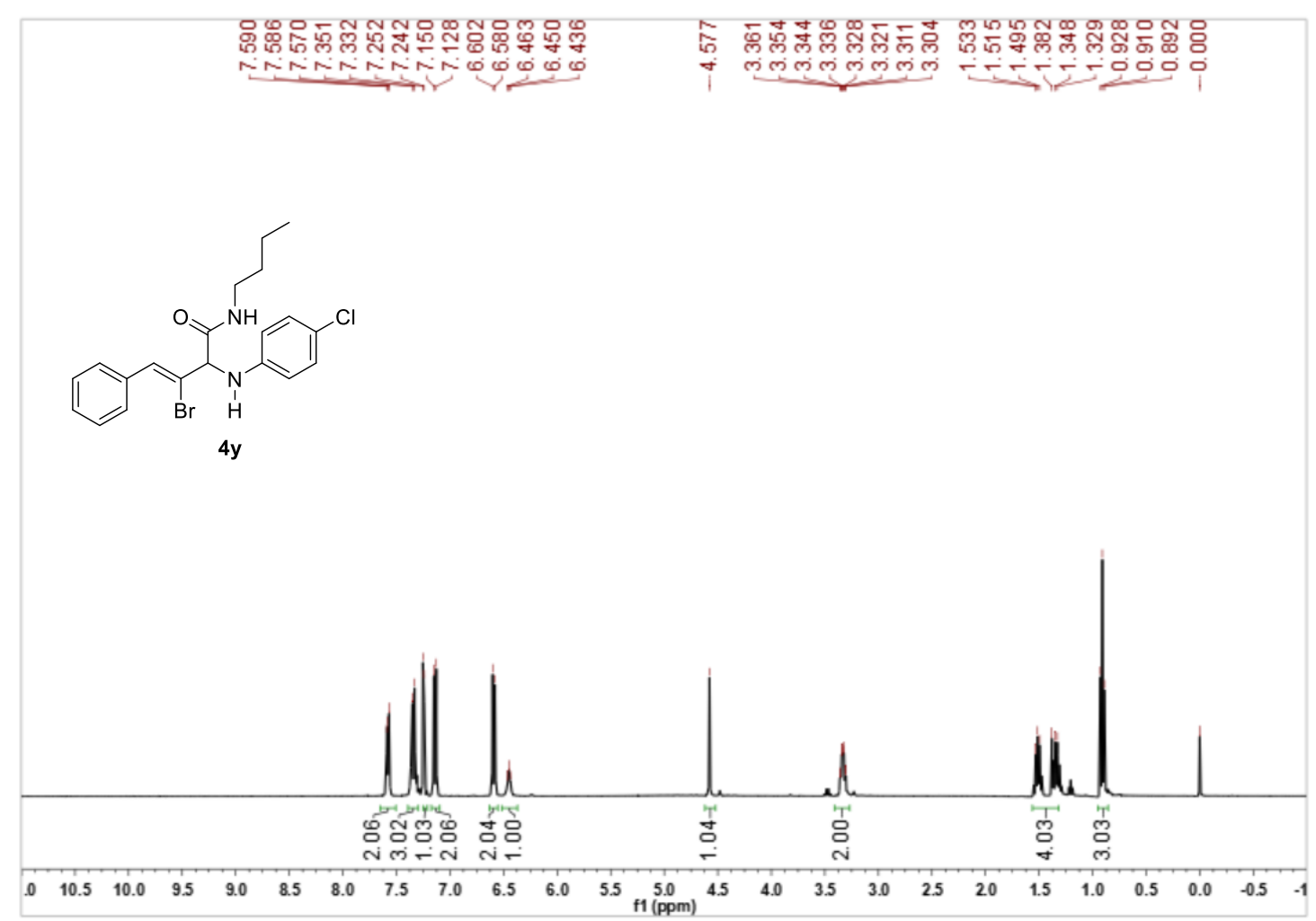

${ }^{1} \mathrm{H}$ NMR (400 MHz), $\mathrm{CDCl}_{3}$

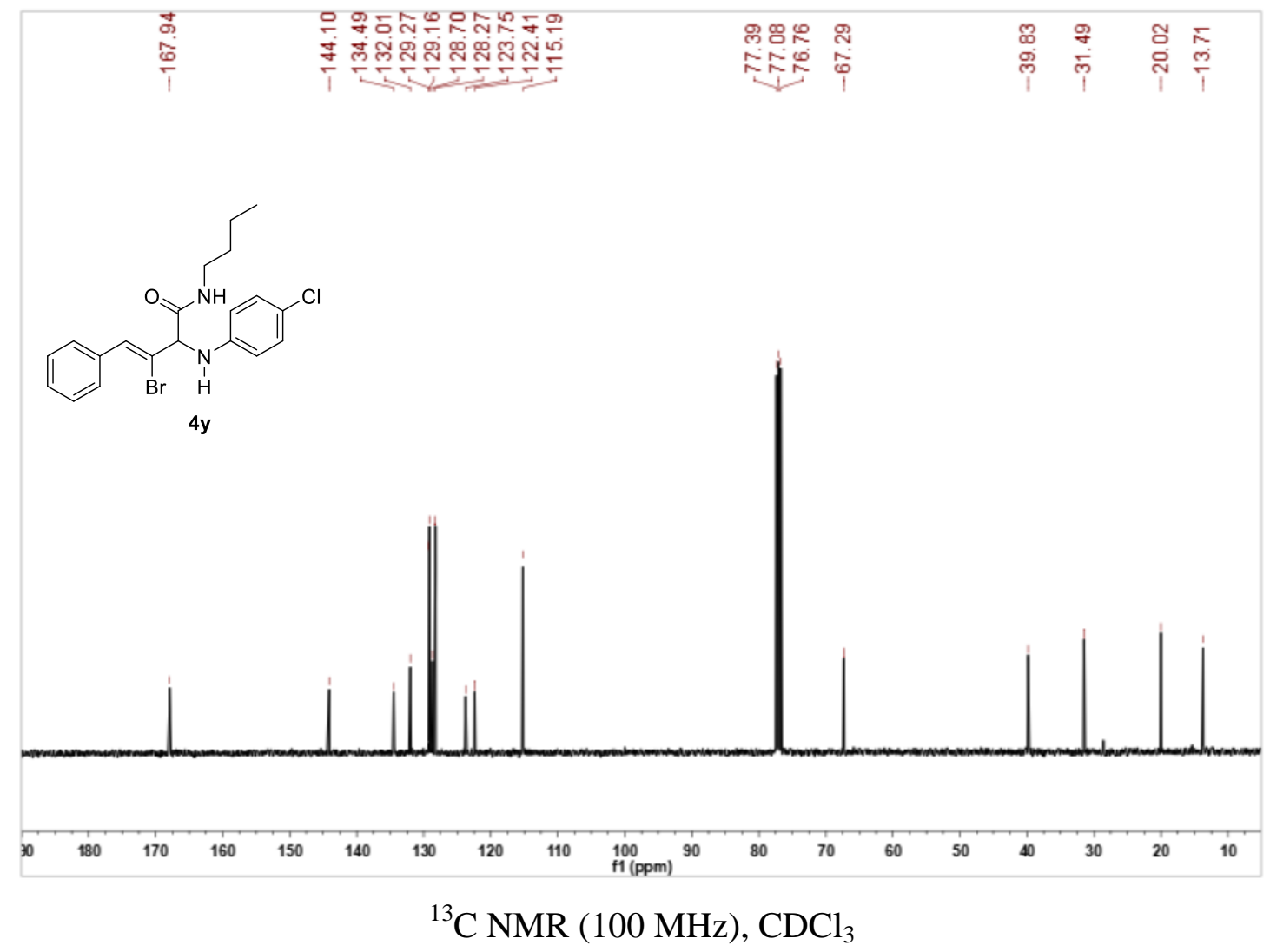




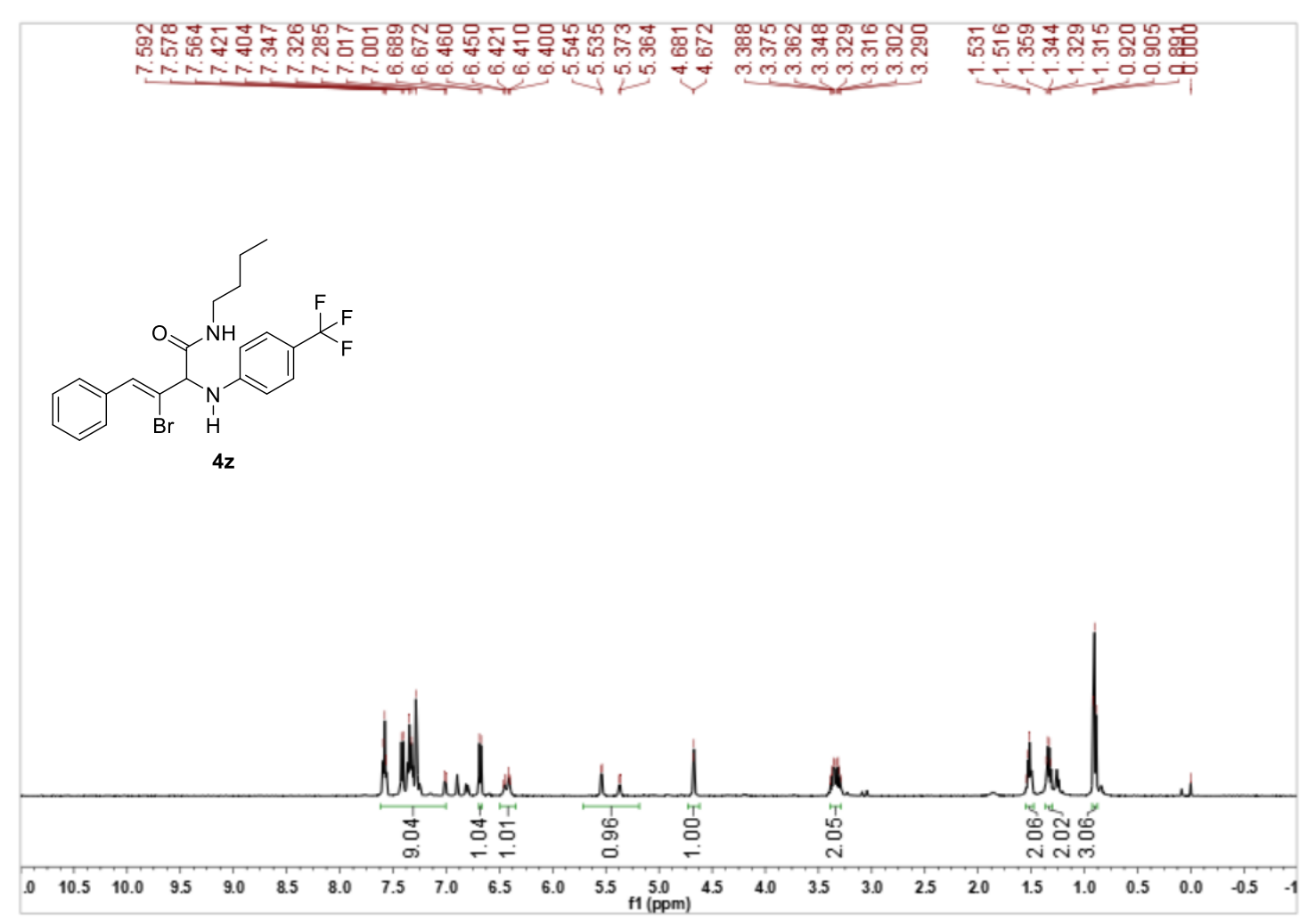

${ }^{1} \mathrm{H}$ NMR $(400 \mathrm{MHz}), \mathrm{CDCl}_{3}$

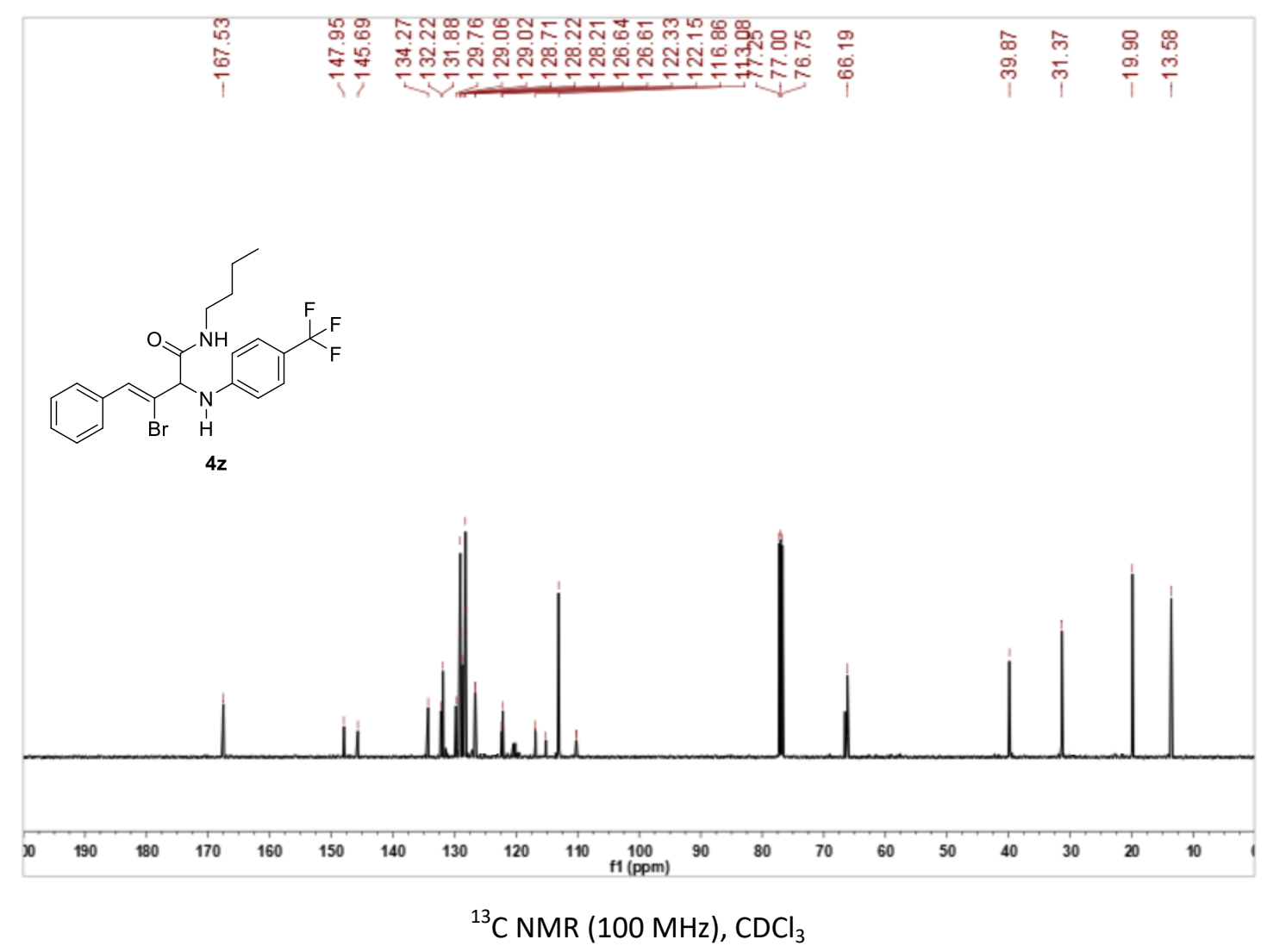




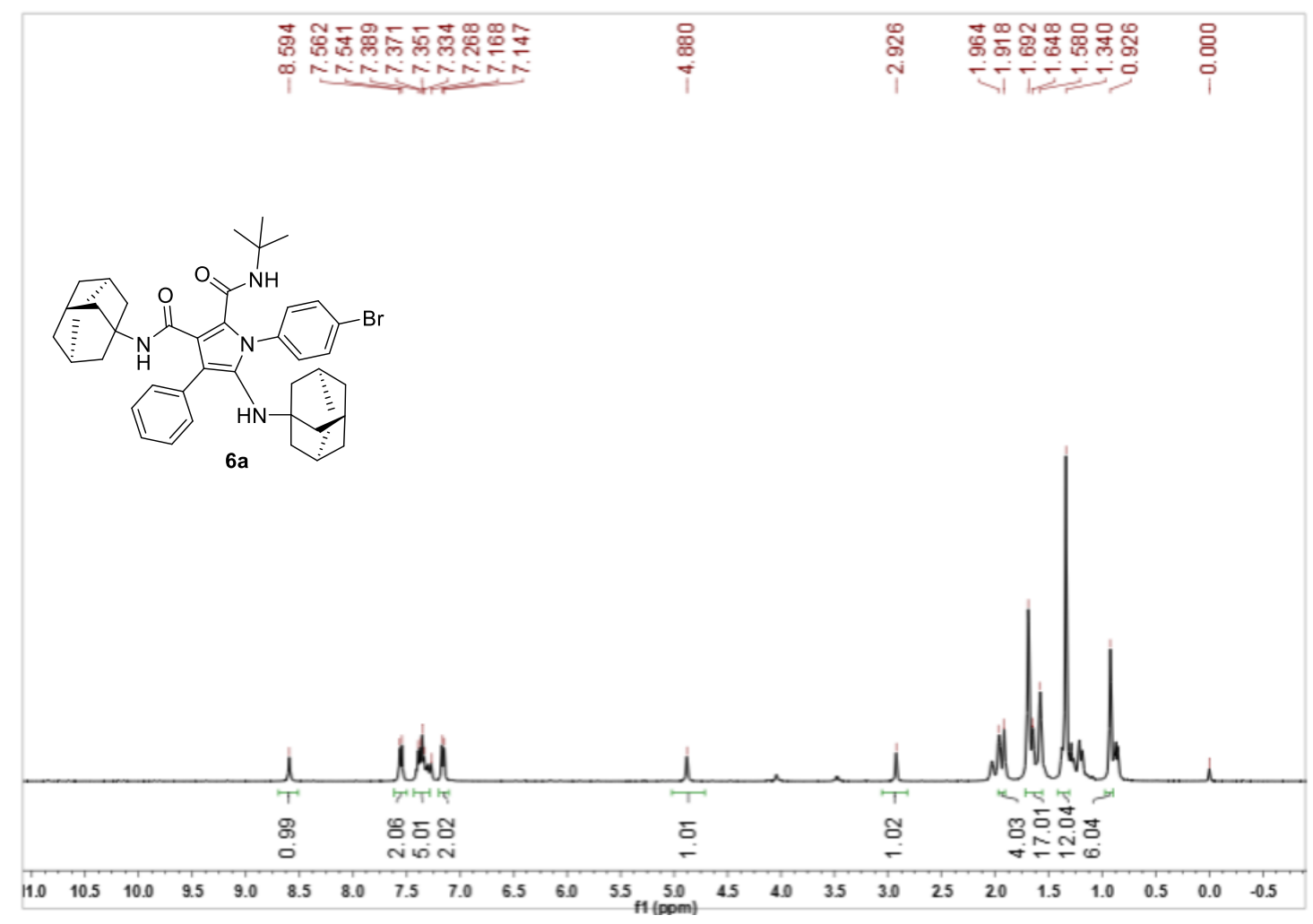

${ }^{1} \mathrm{H}$ NMR (400 MHz), $\mathrm{CDCl}_{3}$

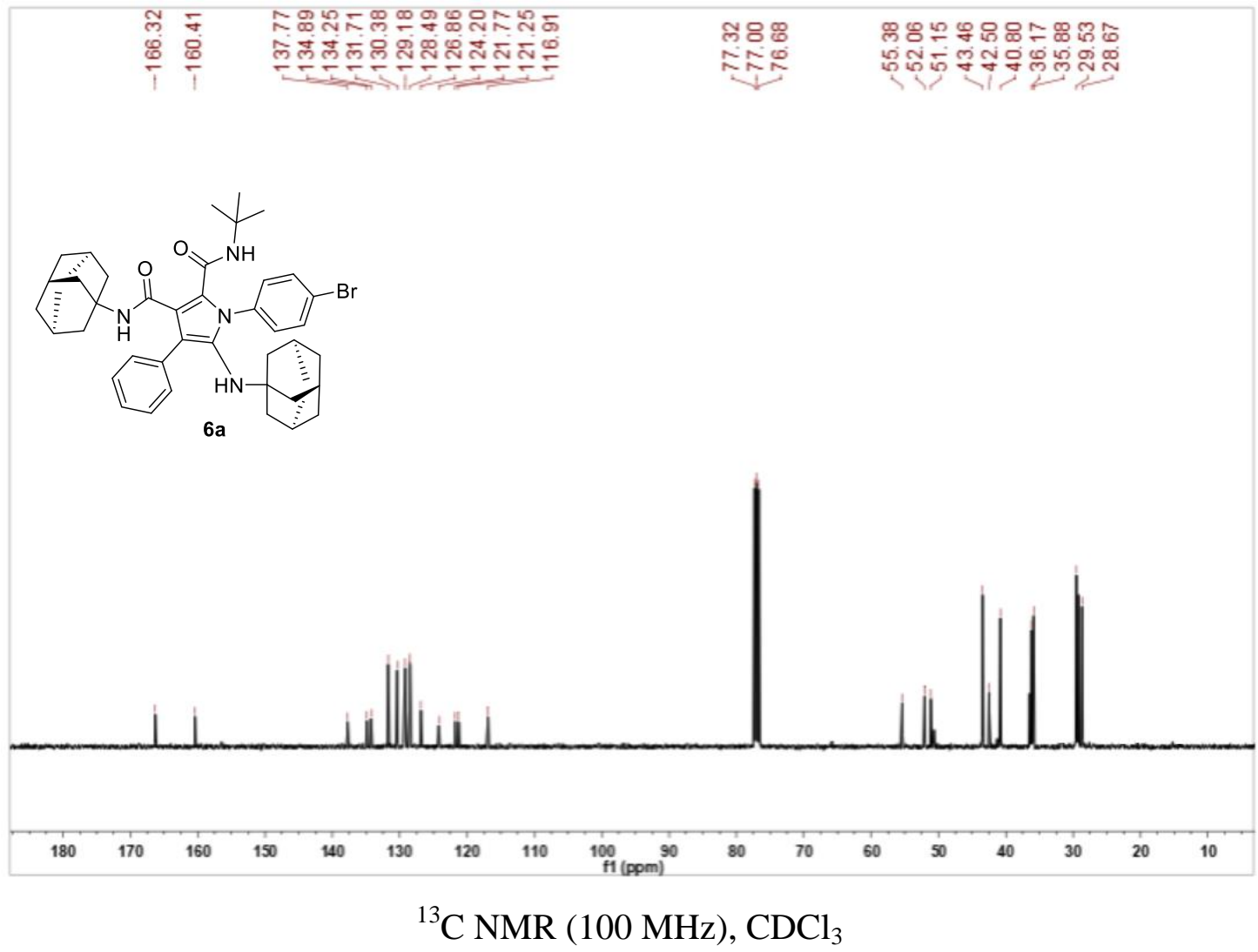




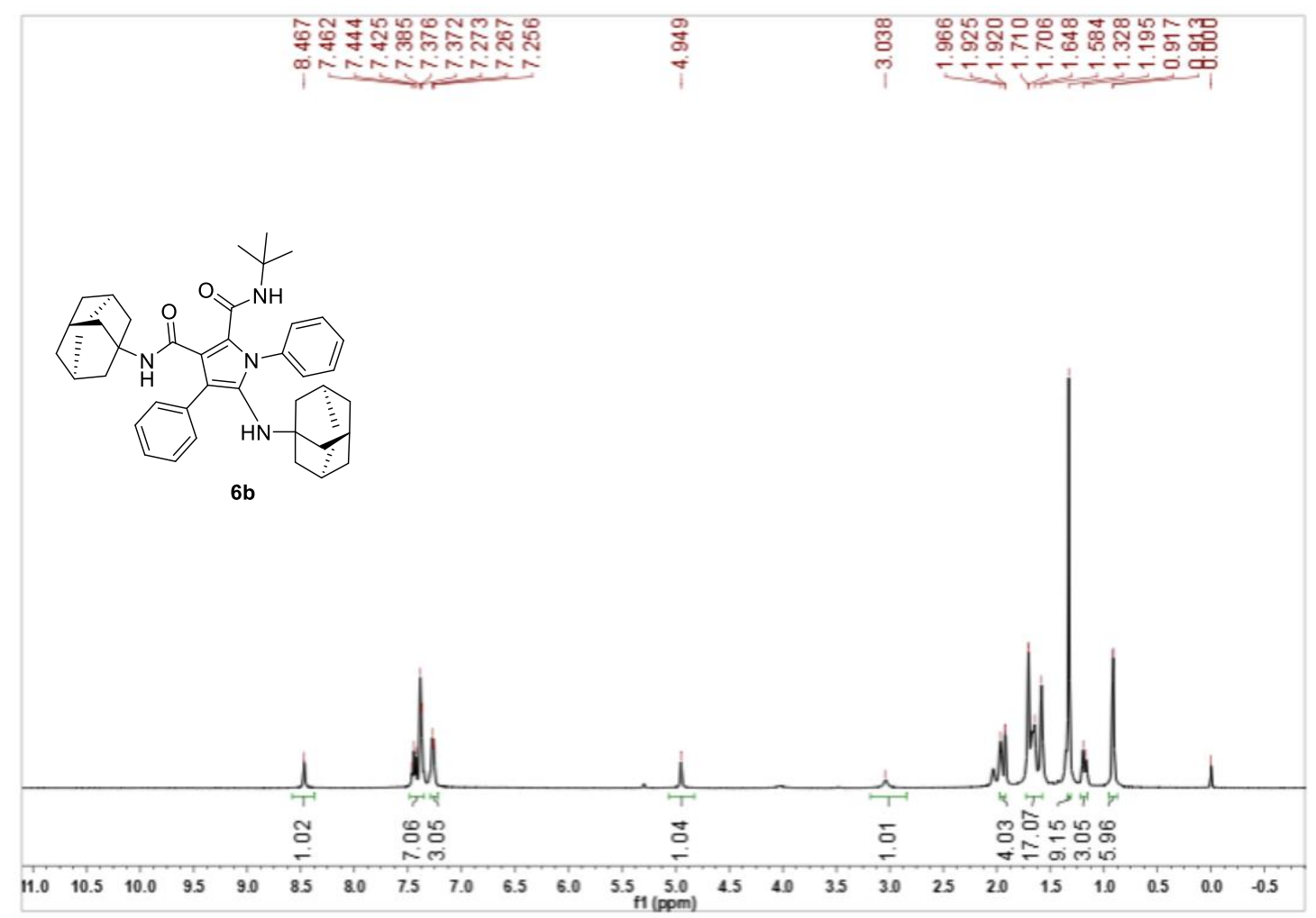

${ }^{1} \mathrm{H}$ NMR (400 MHz), $\mathrm{CDCl}_{3}$

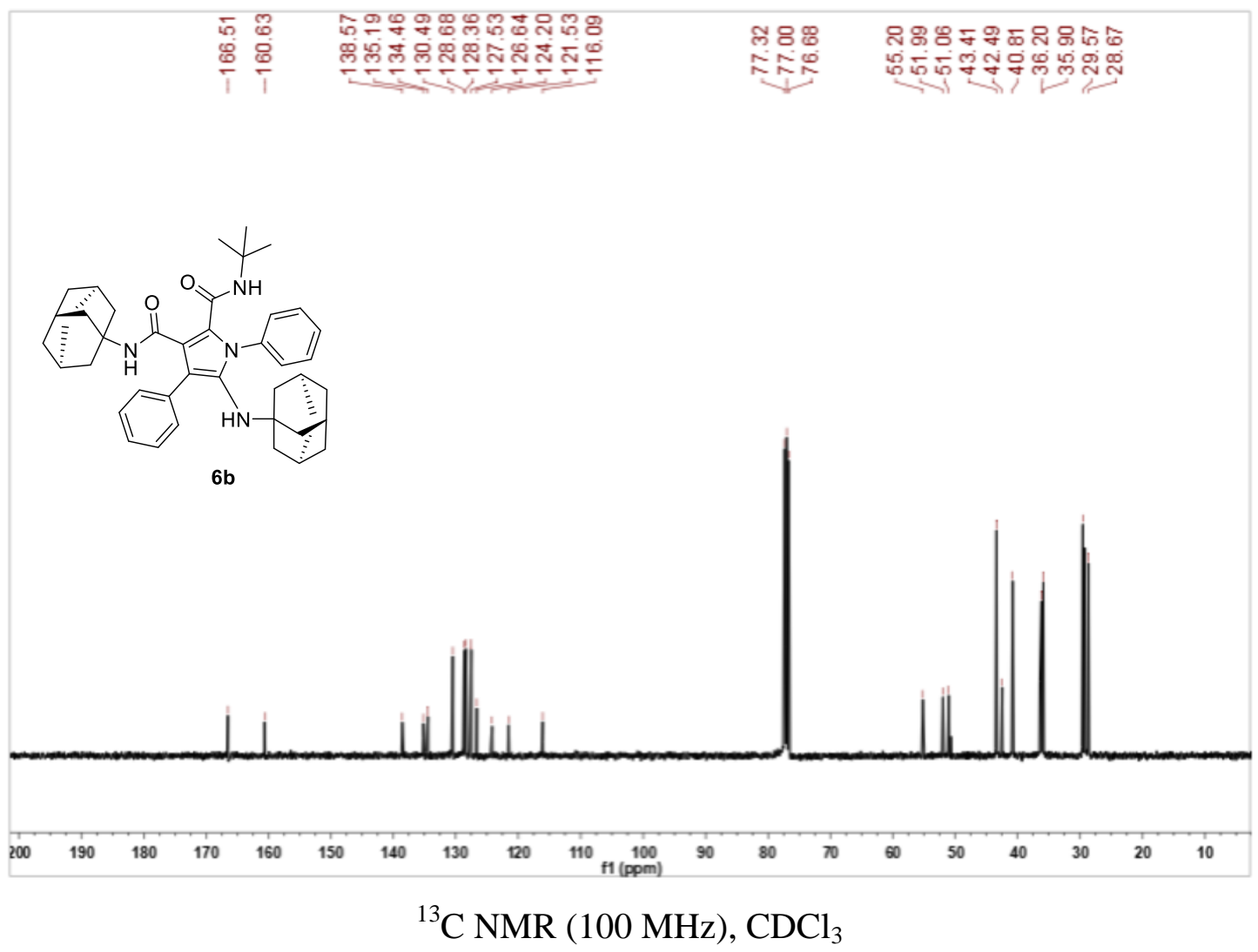




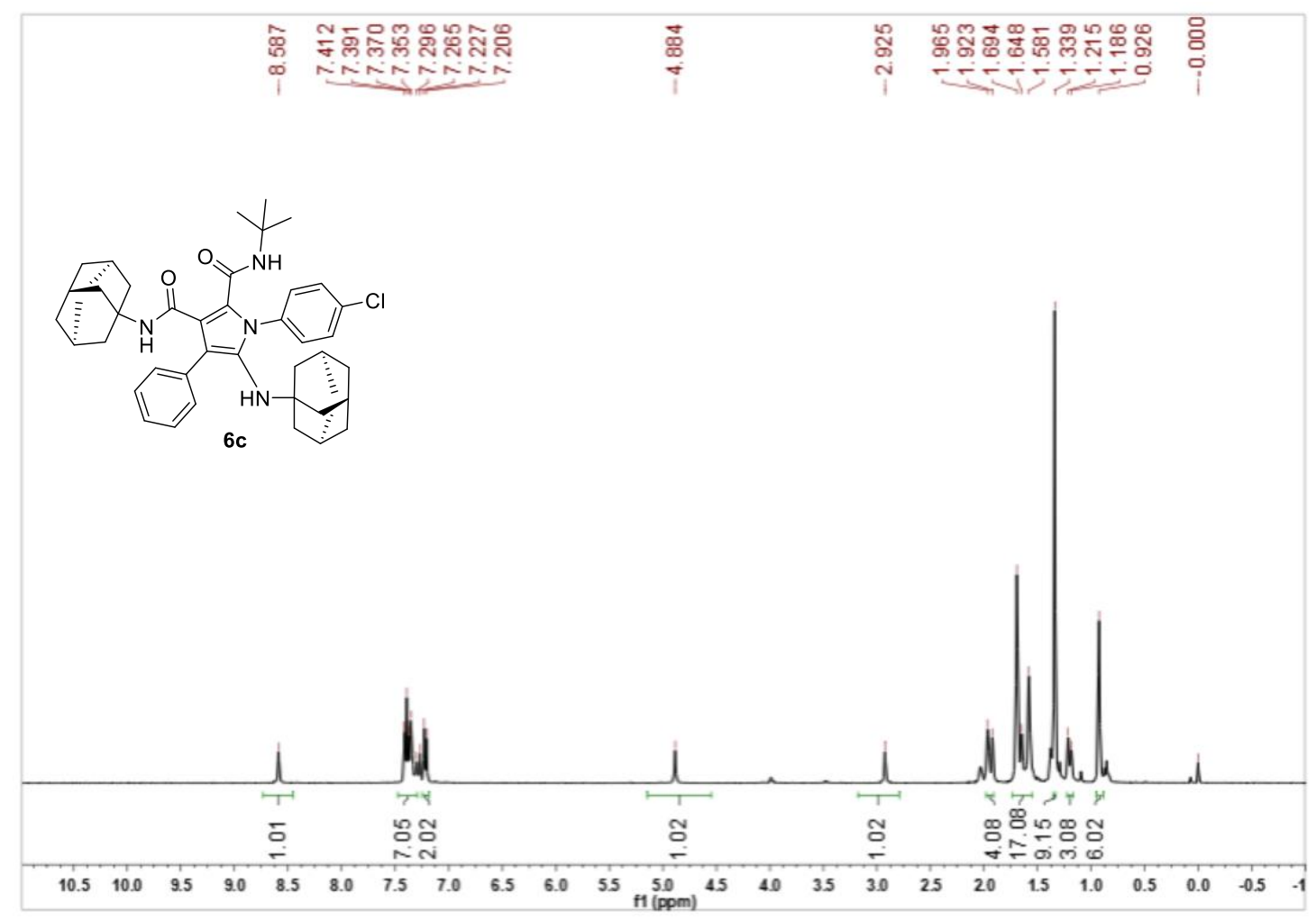

${ }^{1} \mathrm{H}$ NMR (400 MHz), $\mathrm{CDCl}_{3}$

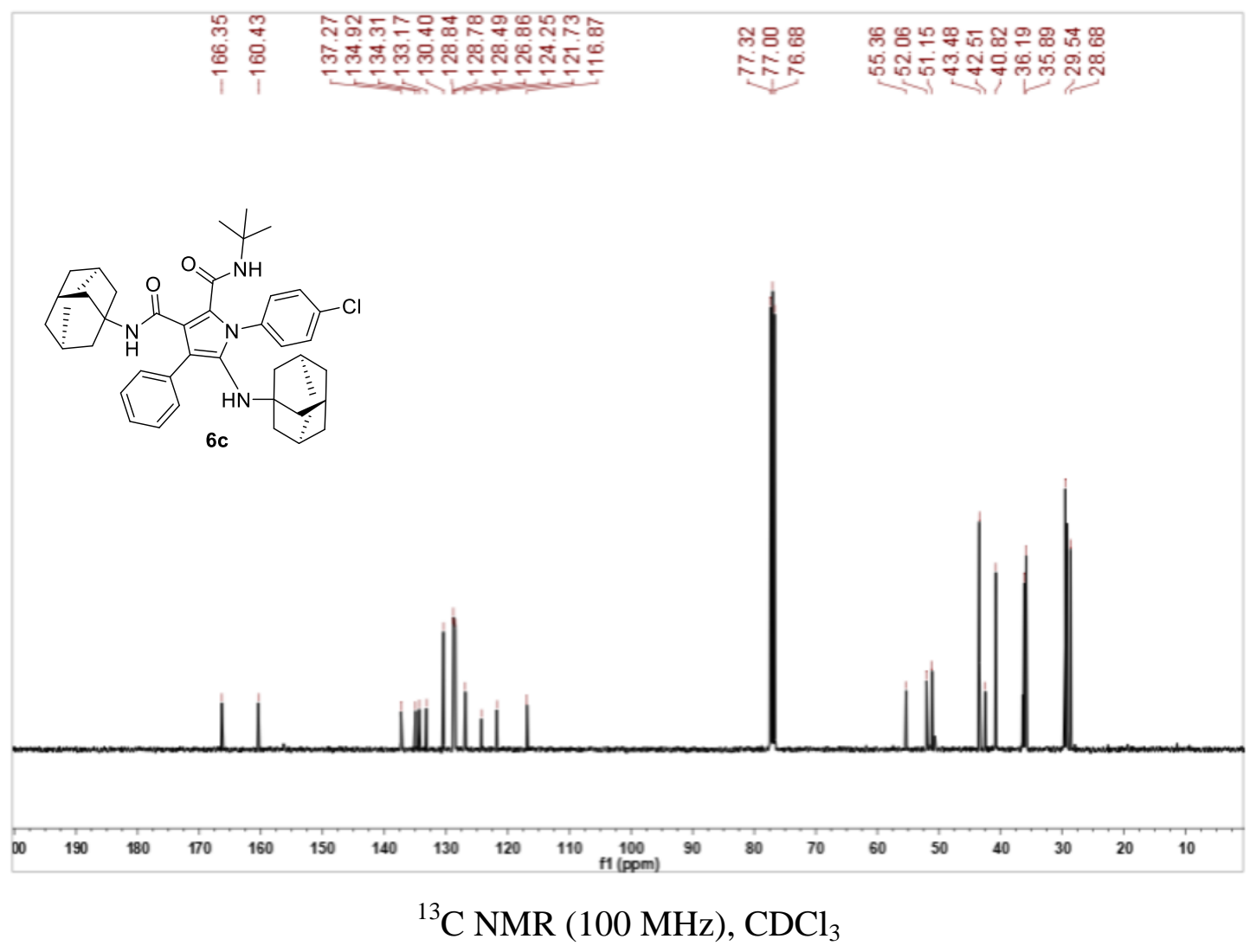




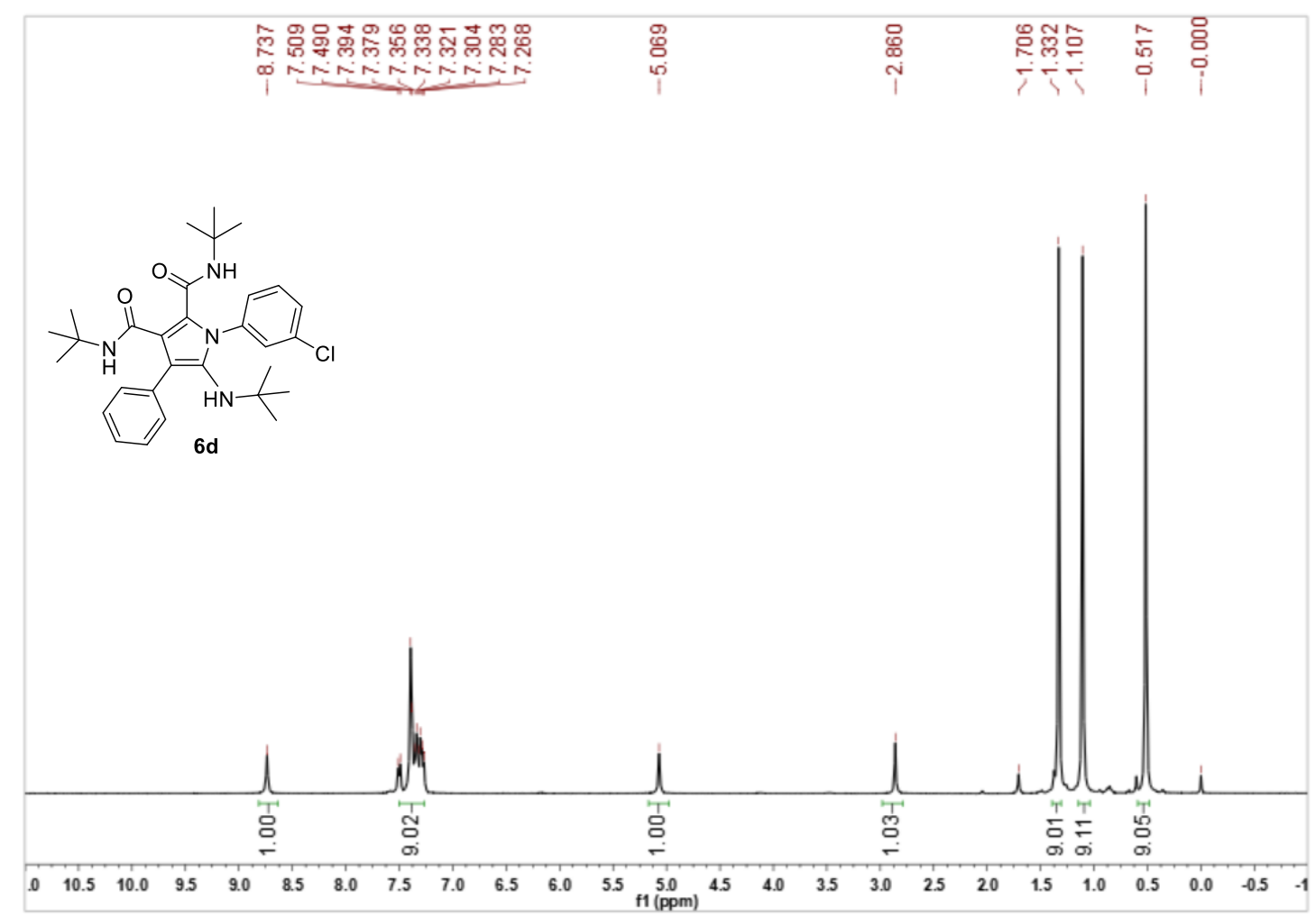

${ }^{1} \mathrm{H}$ NMR (400 MHz), $\mathrm{CDCl}_{3}$

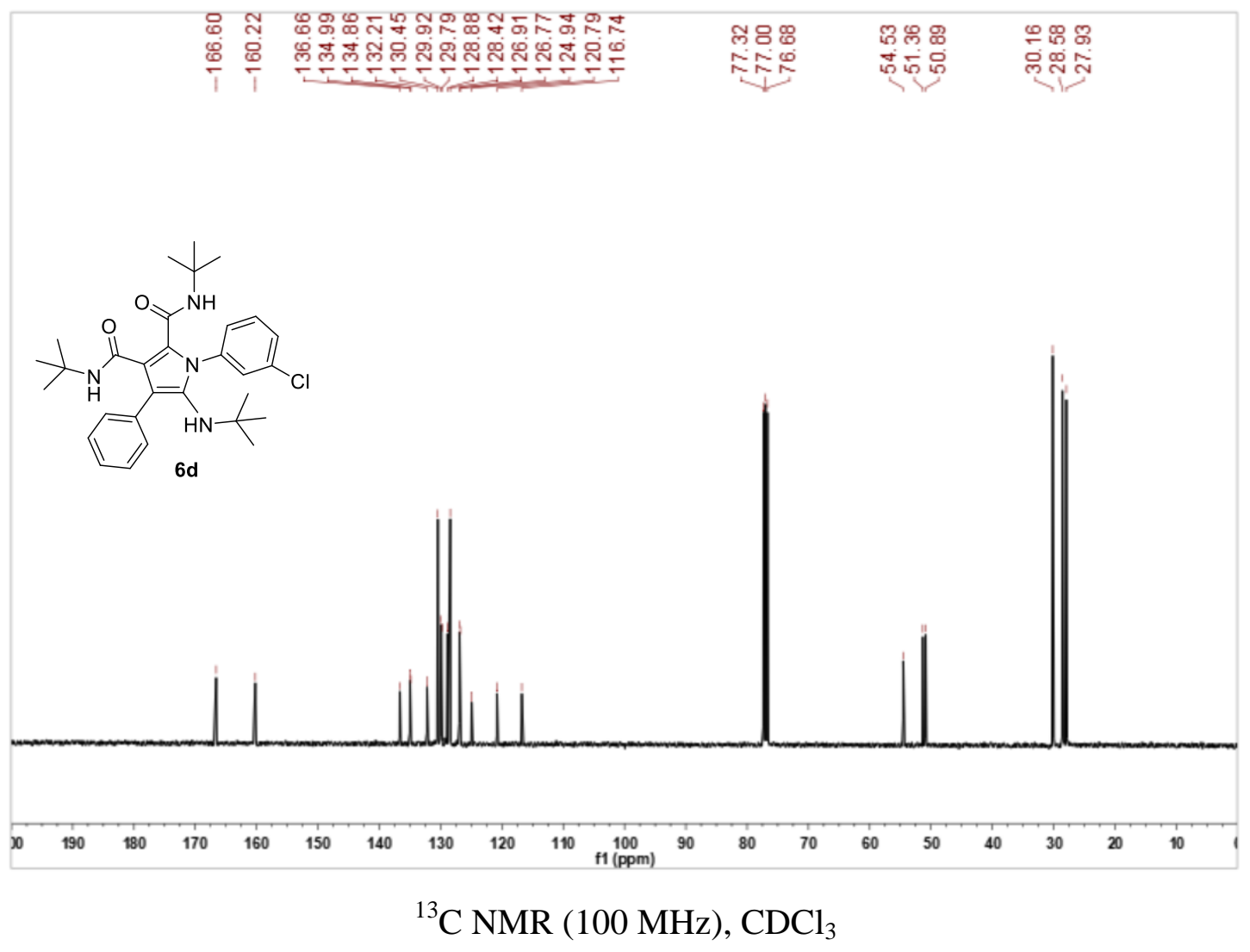




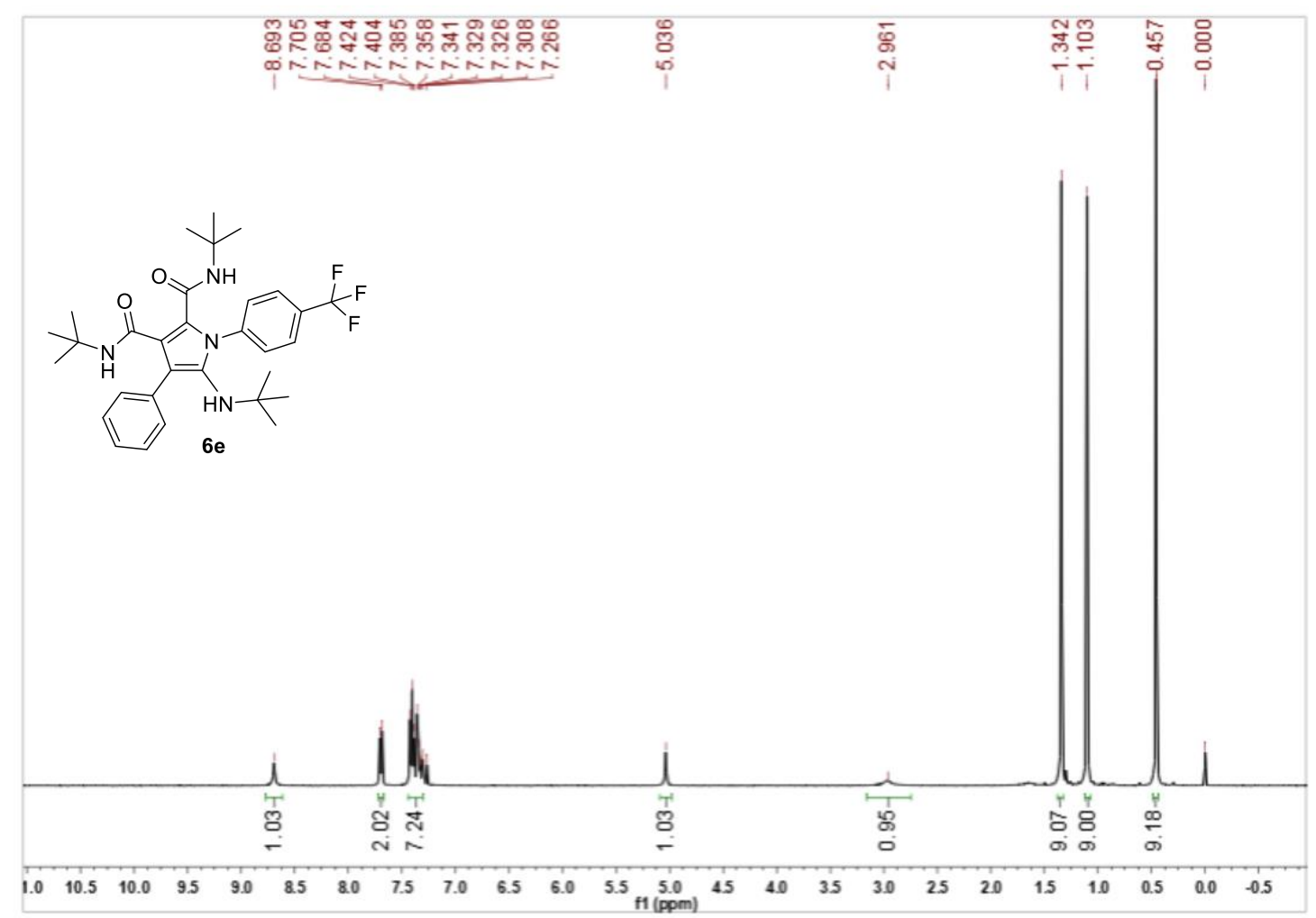

${ }^{1} \mathrm{H}$ NMR (400 MHz), $\mathrm{CDCl}_{3}$

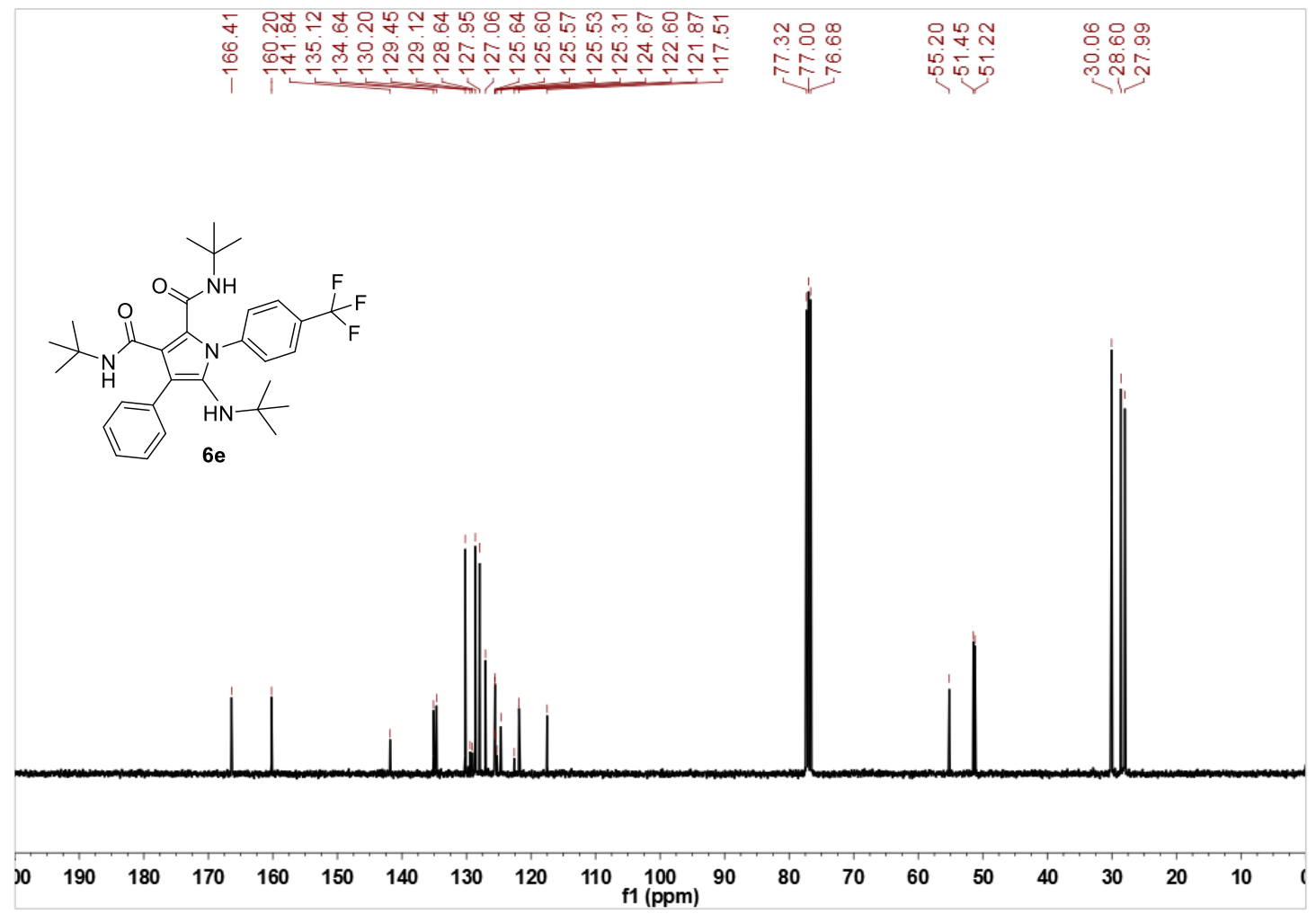

${ }^{13} \mathrm{C}$ NMR (100 MHz), $\mathrm{CDCl}_{3}$ 


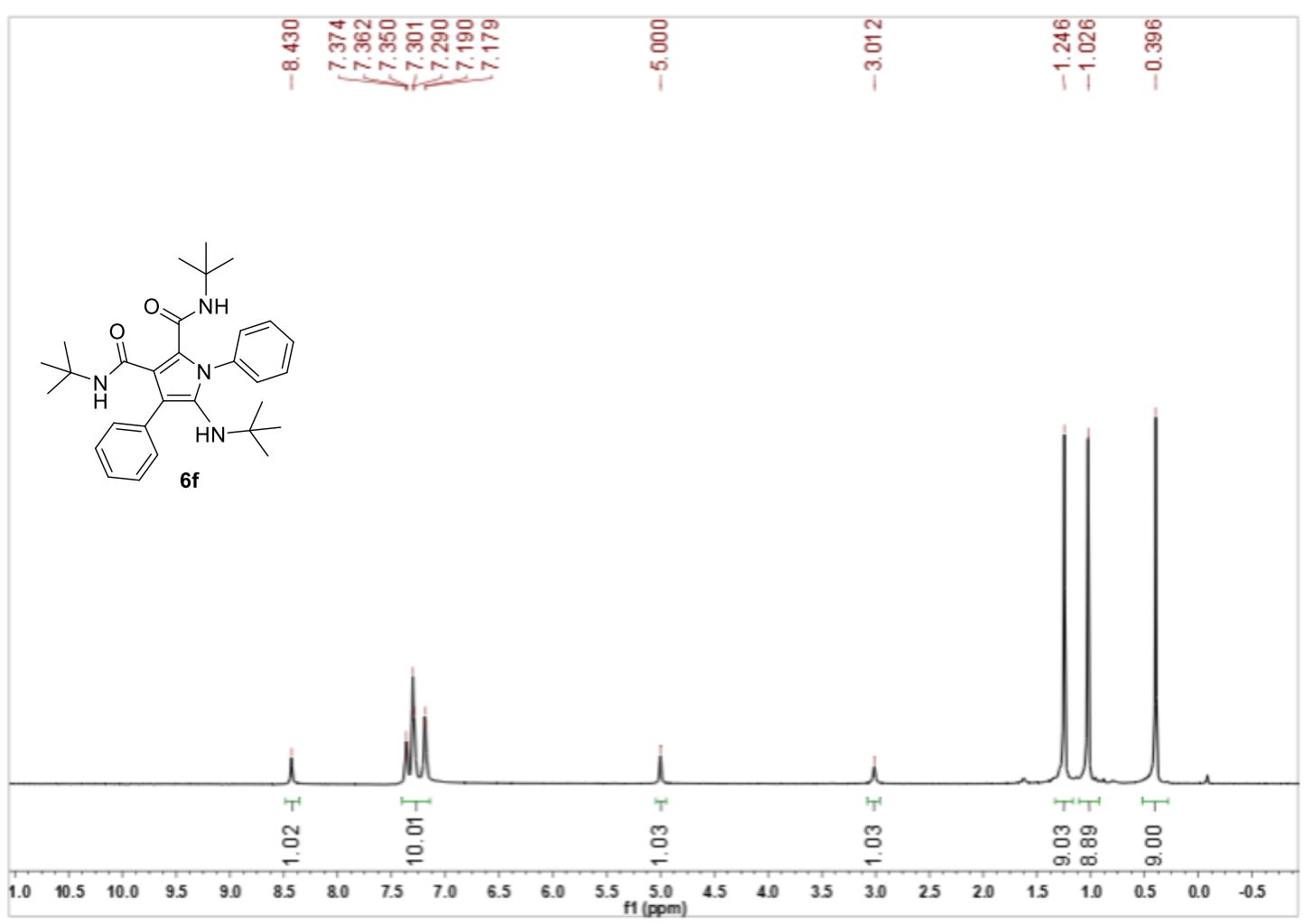

${ }^{1} \mathrm{H}$ NMR (400 MHz), $\mathrm{CDCl}_{3}$

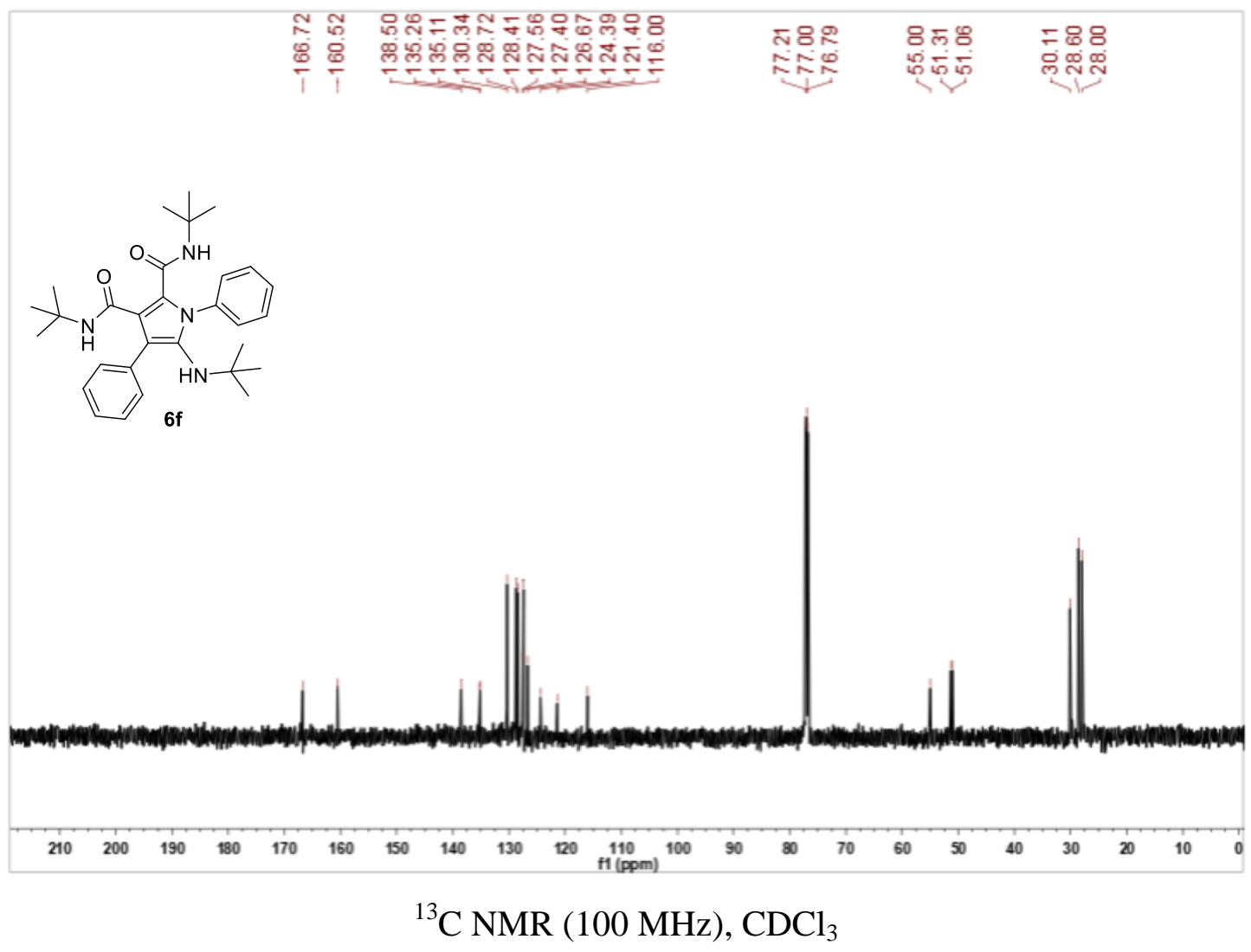




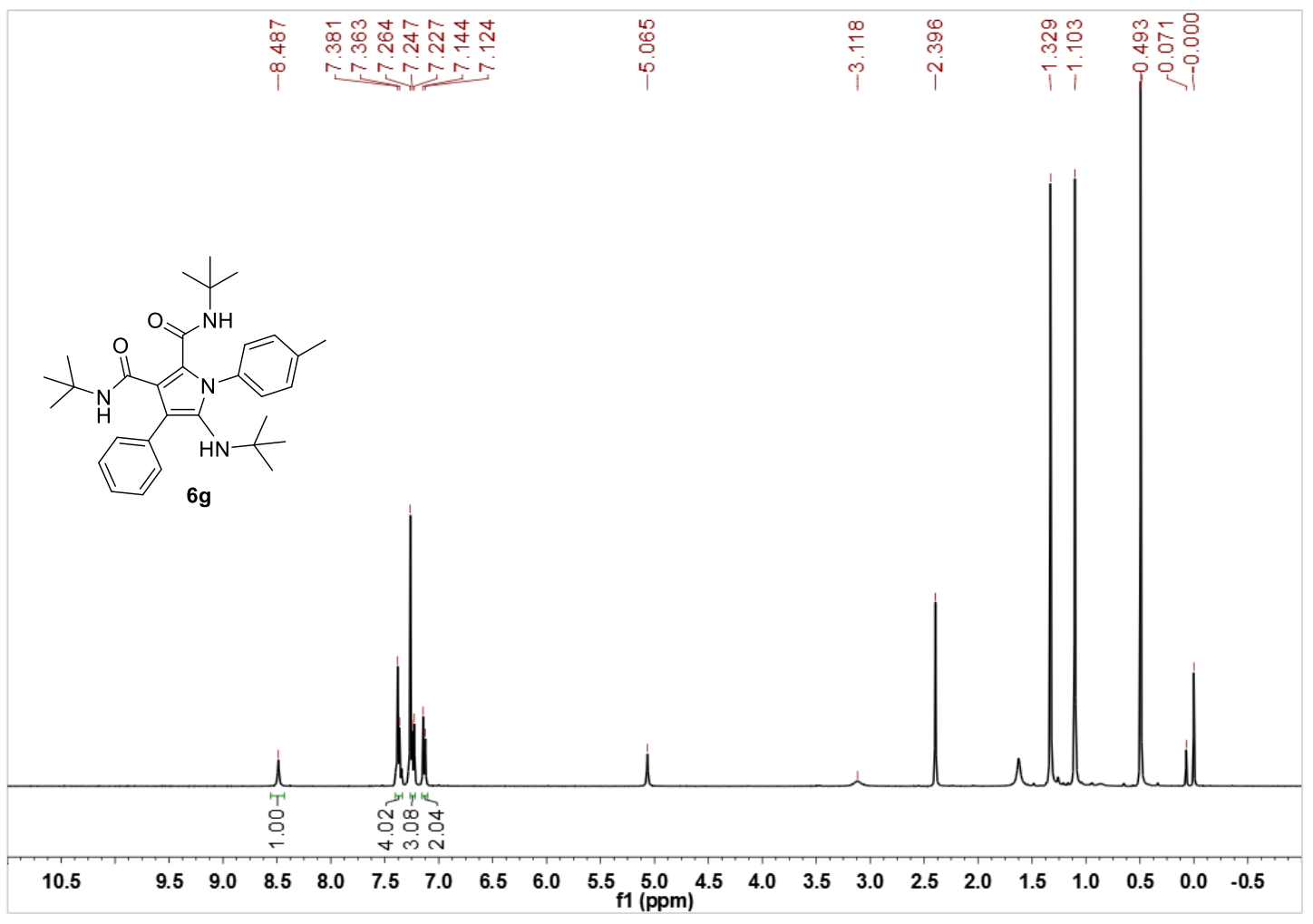

${ }^{1} \mathrm{H}$ NMR (400 MHz), $\mathrm{CDCl}_{3}$

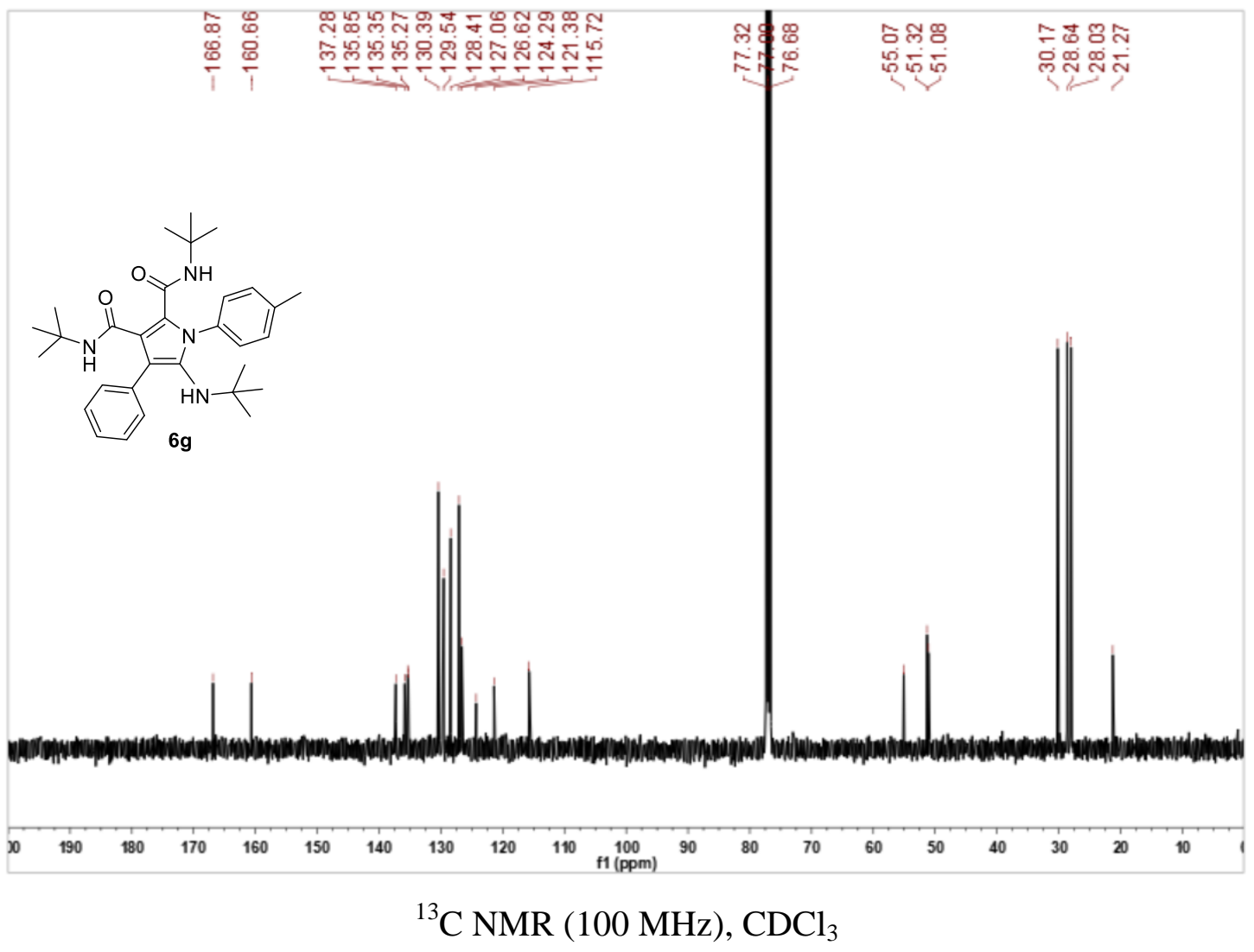




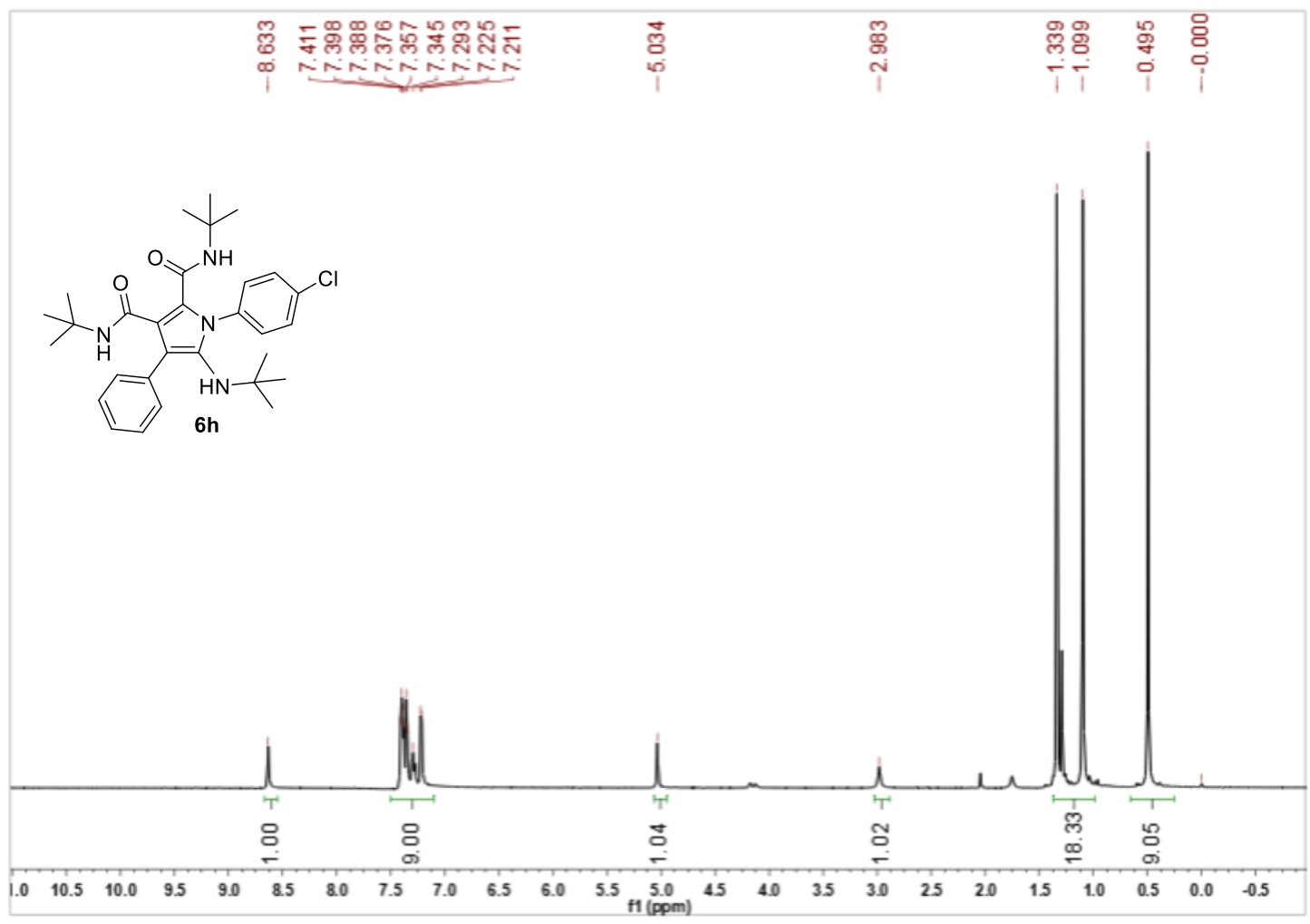

${ }^{1} \mathrm{H}$ NMR (400 MHz), $\mathrm{CDCl}_{3}$

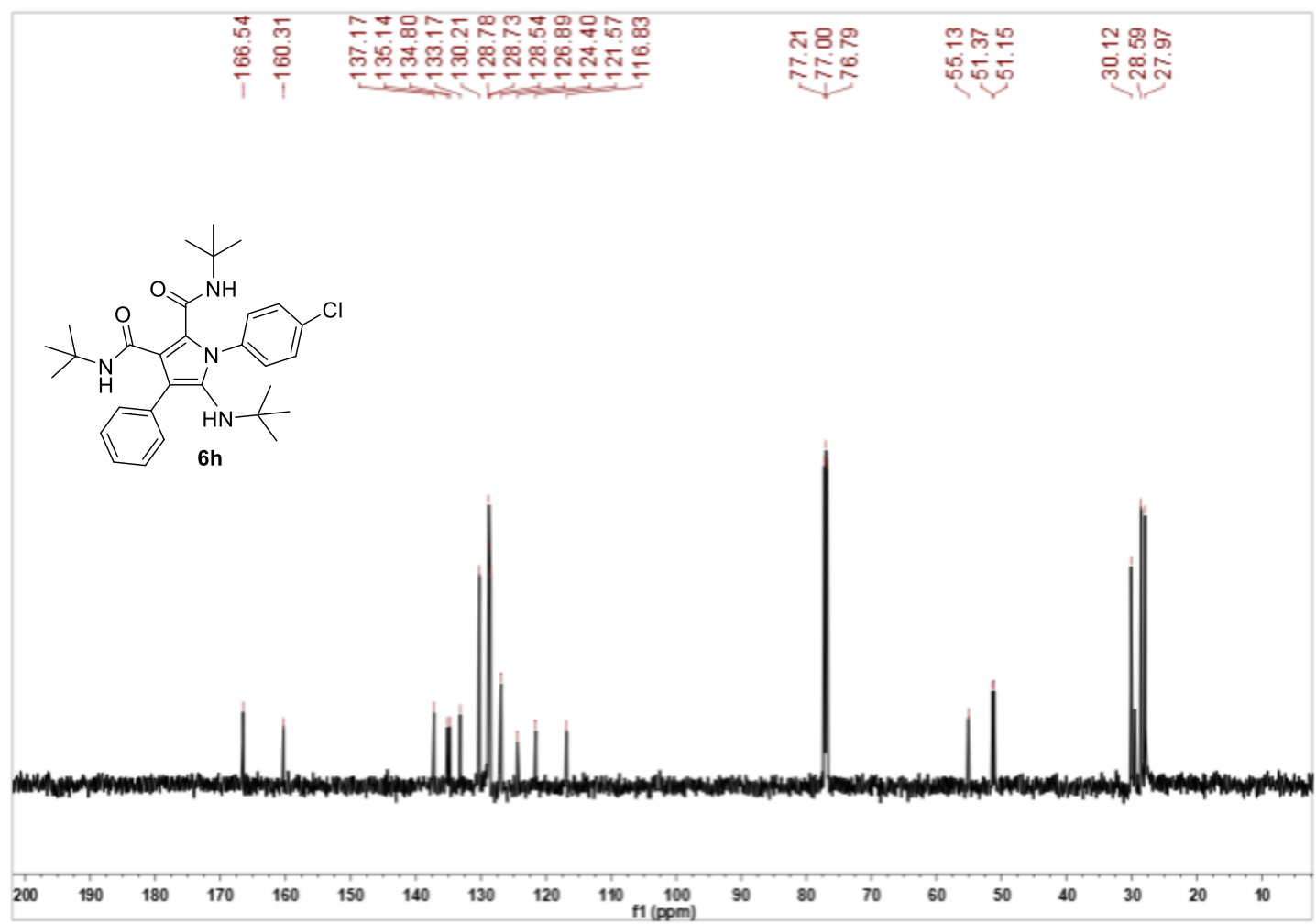

${ }^{13} \mathrm{C} \mathrm{NMR}(100 \mathrm{MHz}), \mathrm{CDCl}_{3}$ 


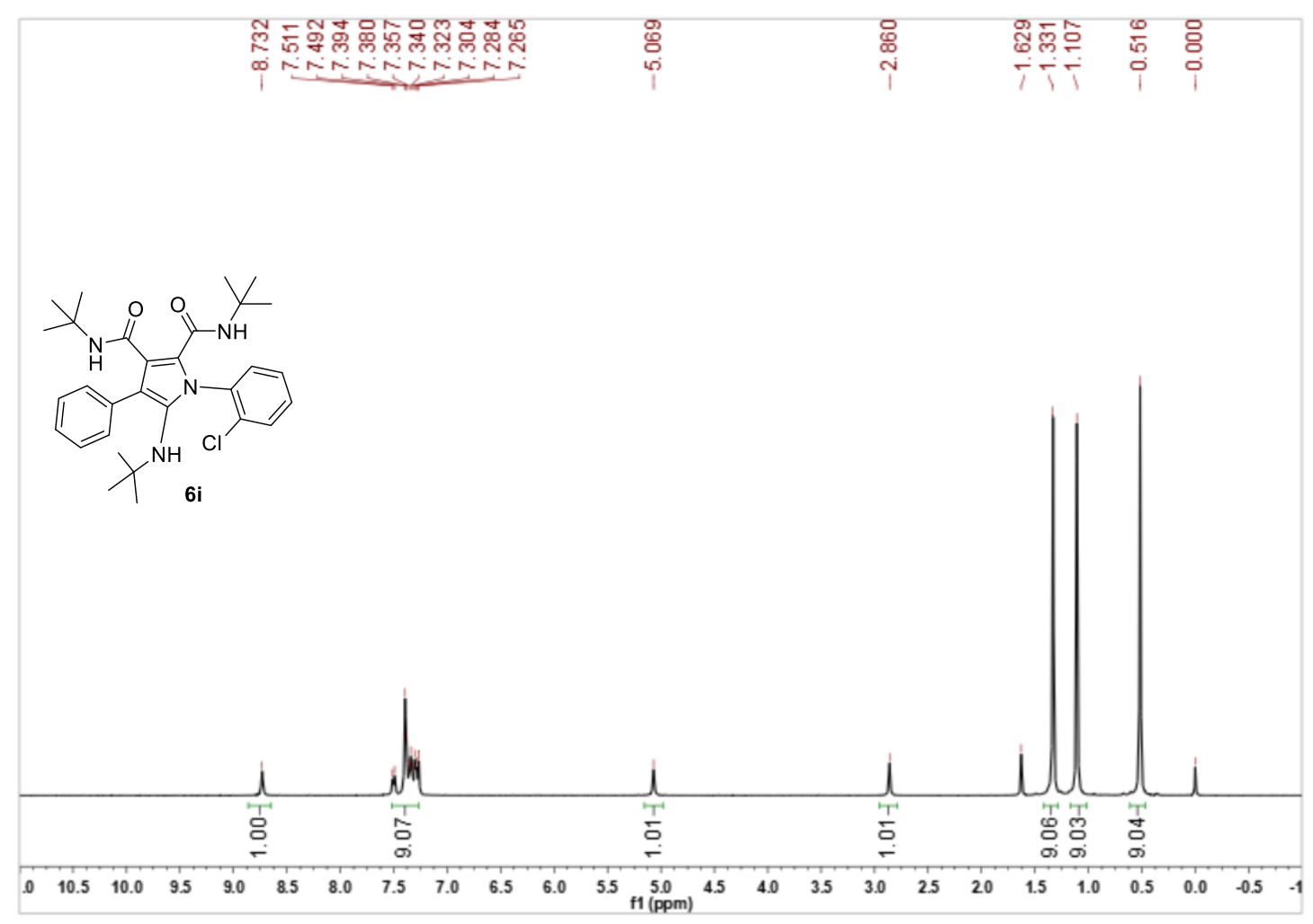

${ }^{1} \mathrm{H}$ NMR (400 MHz), $\mathrm{CDCl}_{3}$

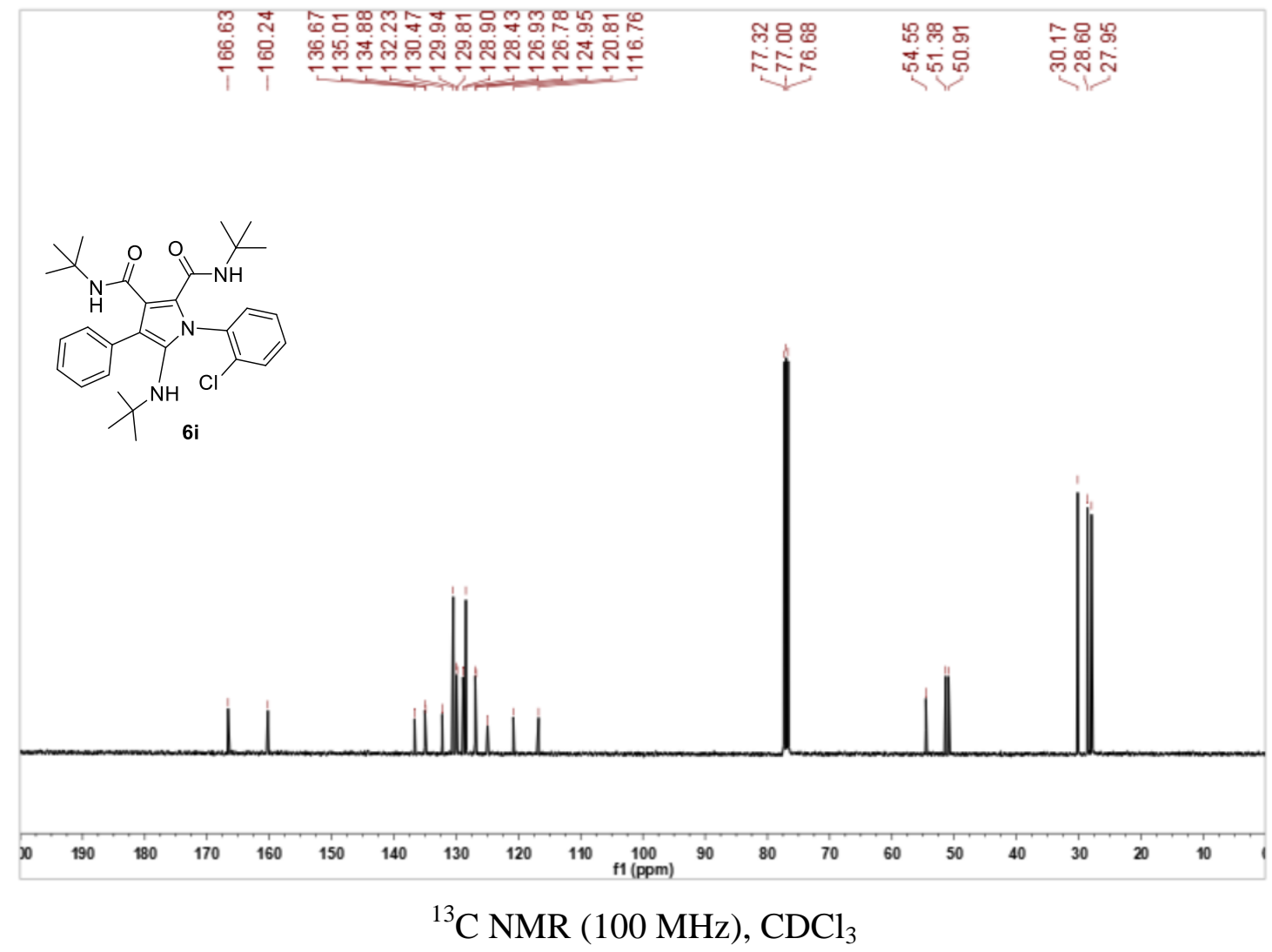




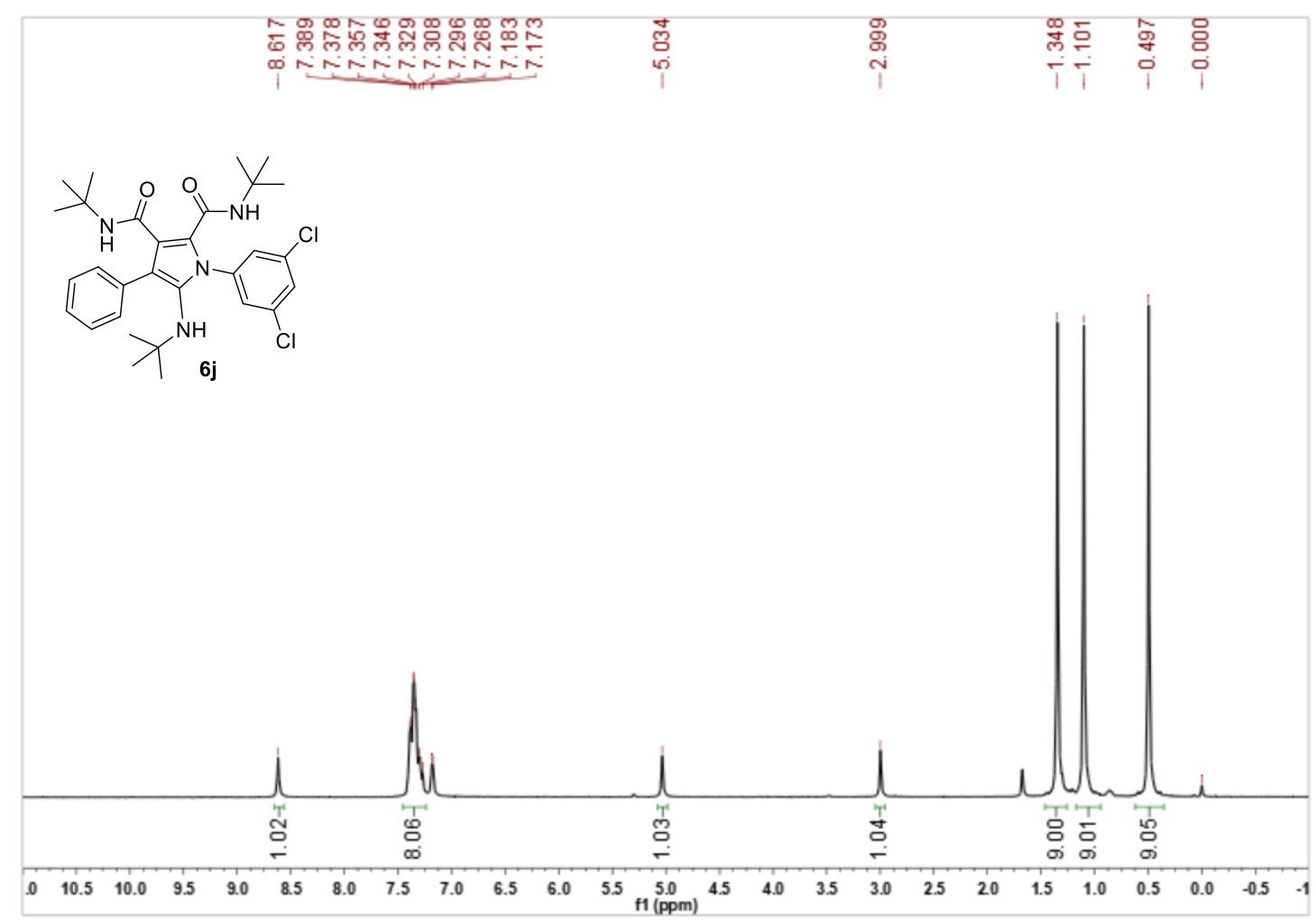

${ }^{1} \mathrm{H}$ NMR (400 MHz), $\mathrm{CDCl}_{3}$

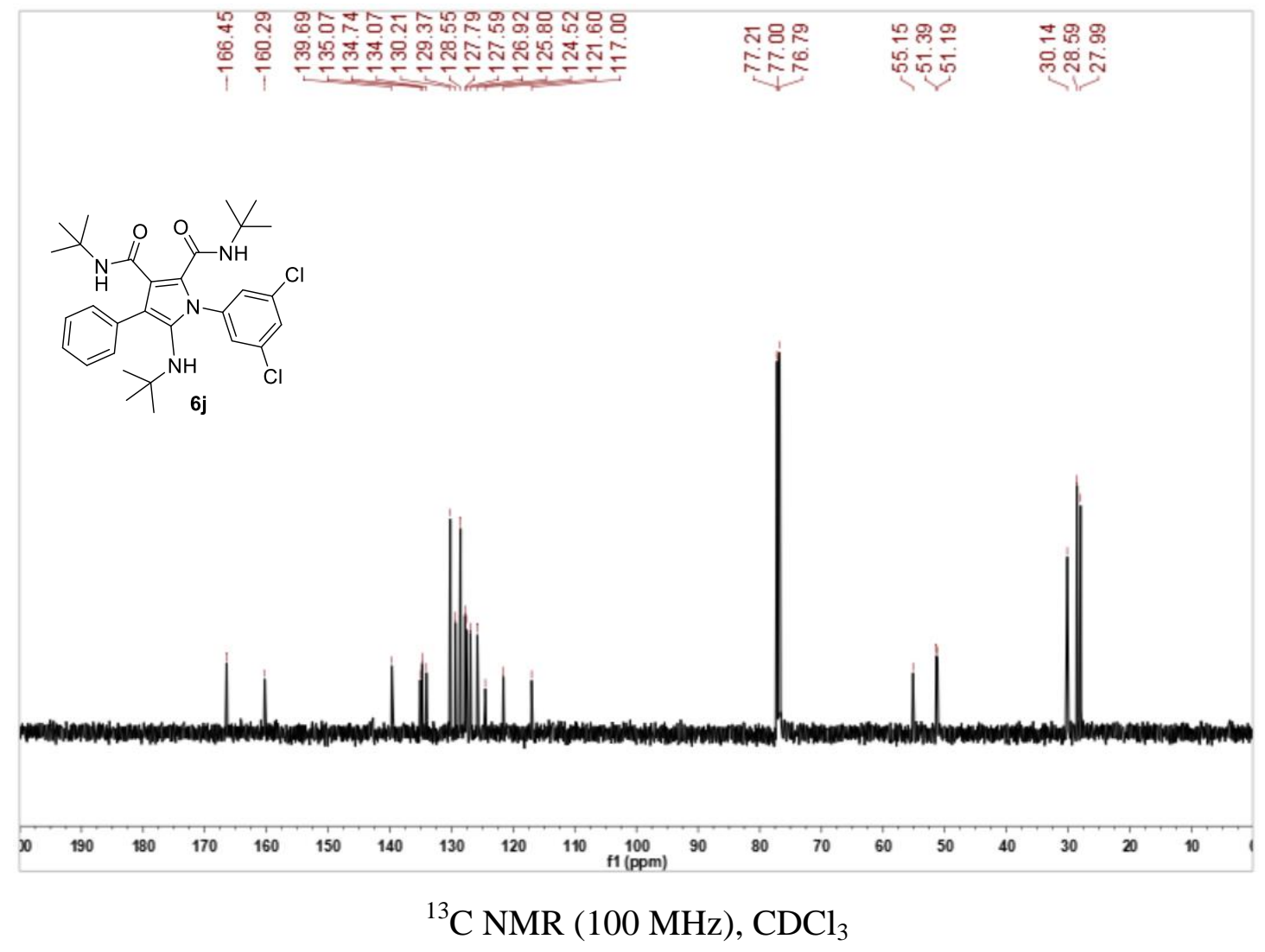




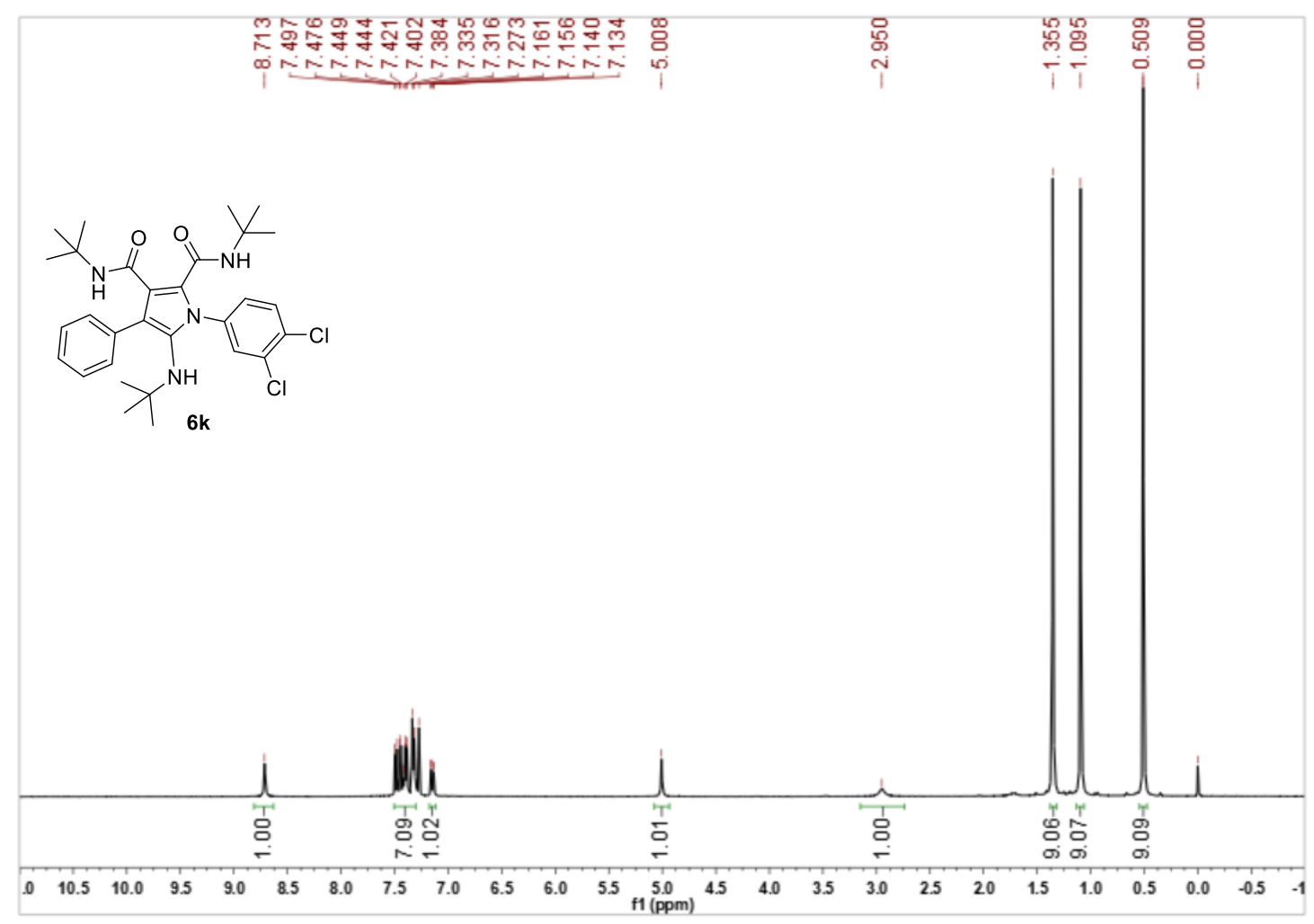

${ }^{1} \mathrm{H}$ NMR (400 MHz), $\mathrm{CDCl}_{3}$

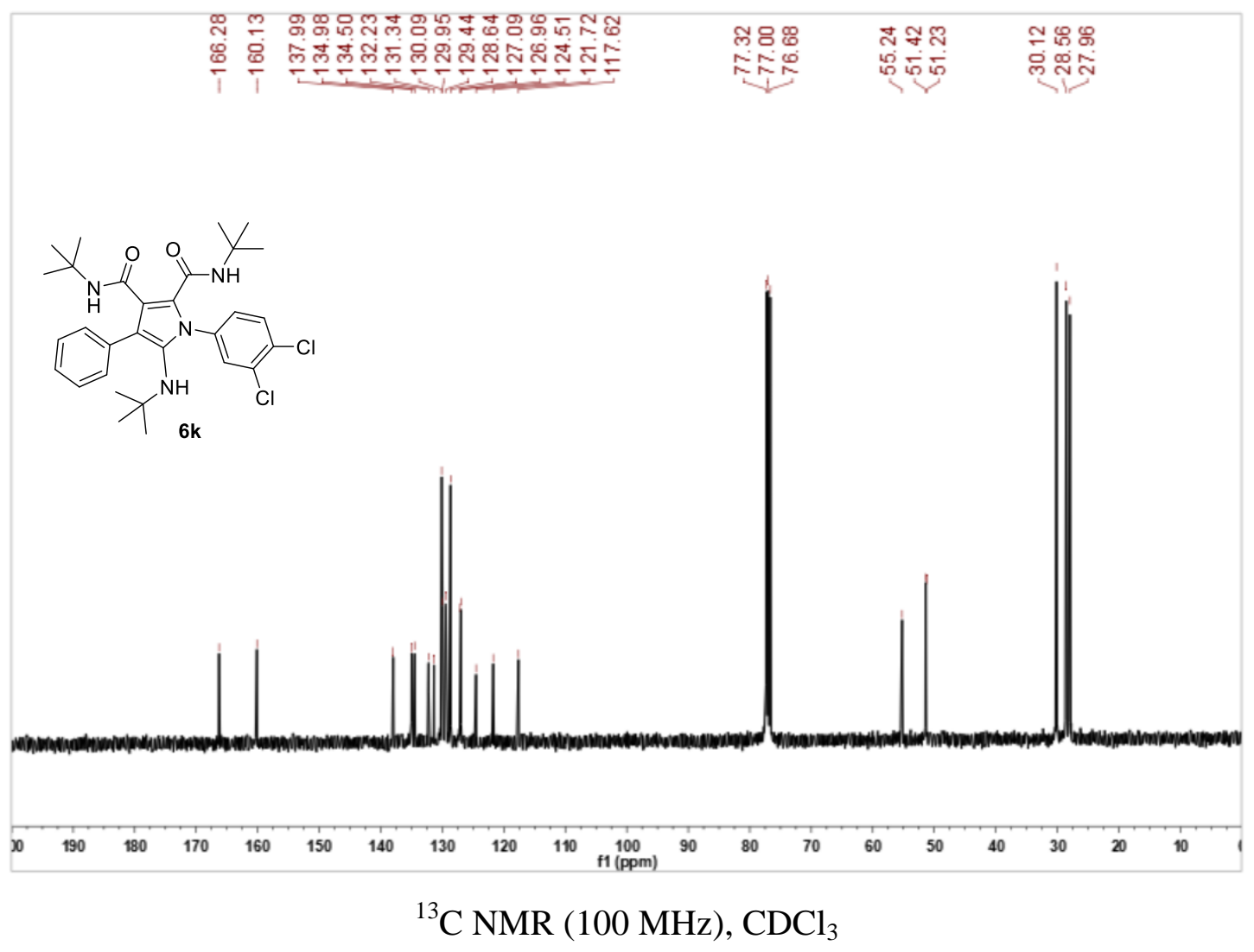




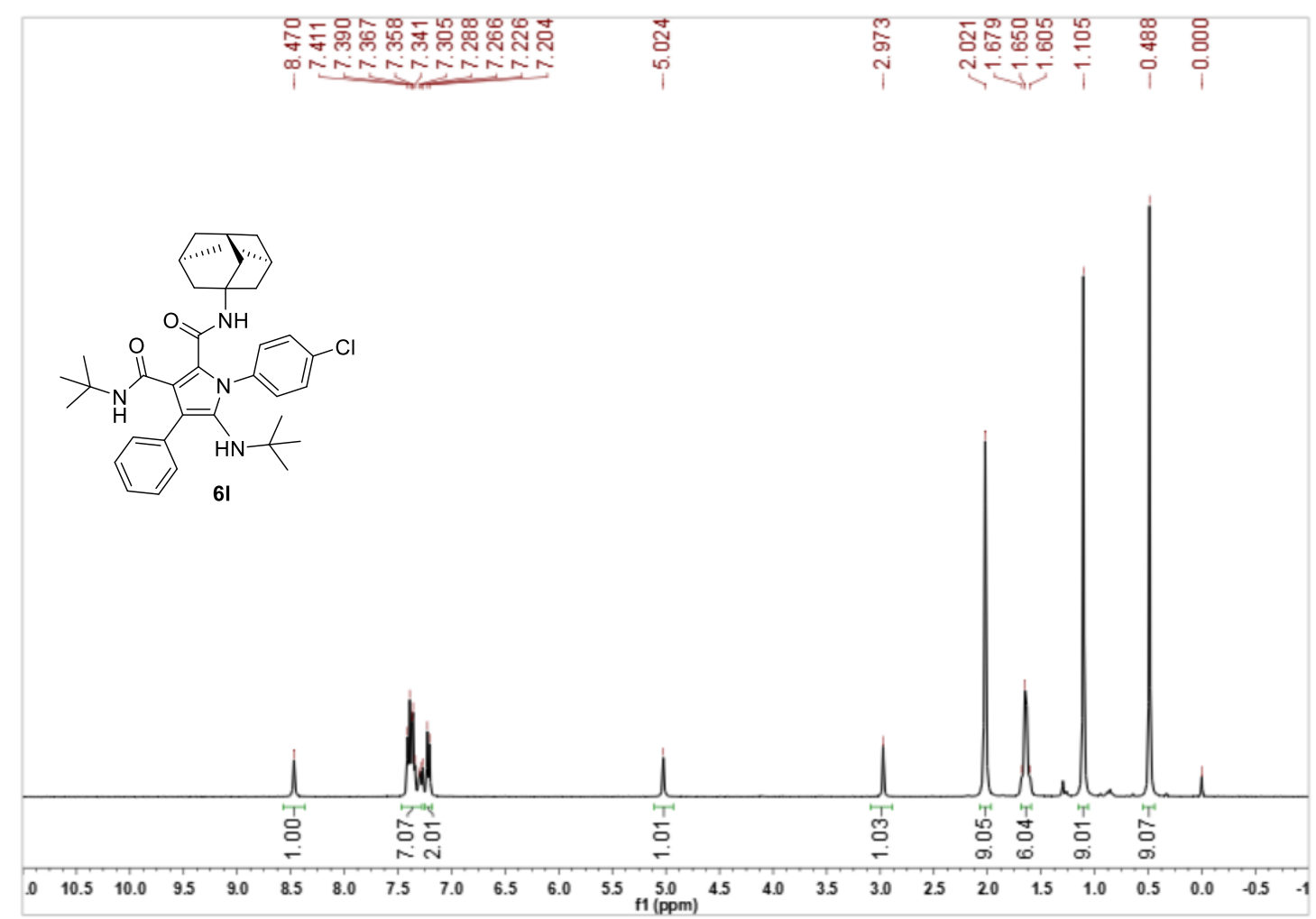

${ }^{1} \mathrm{H}$ NMR (400 MHz), $\mathrm{CDCl}_{3}$

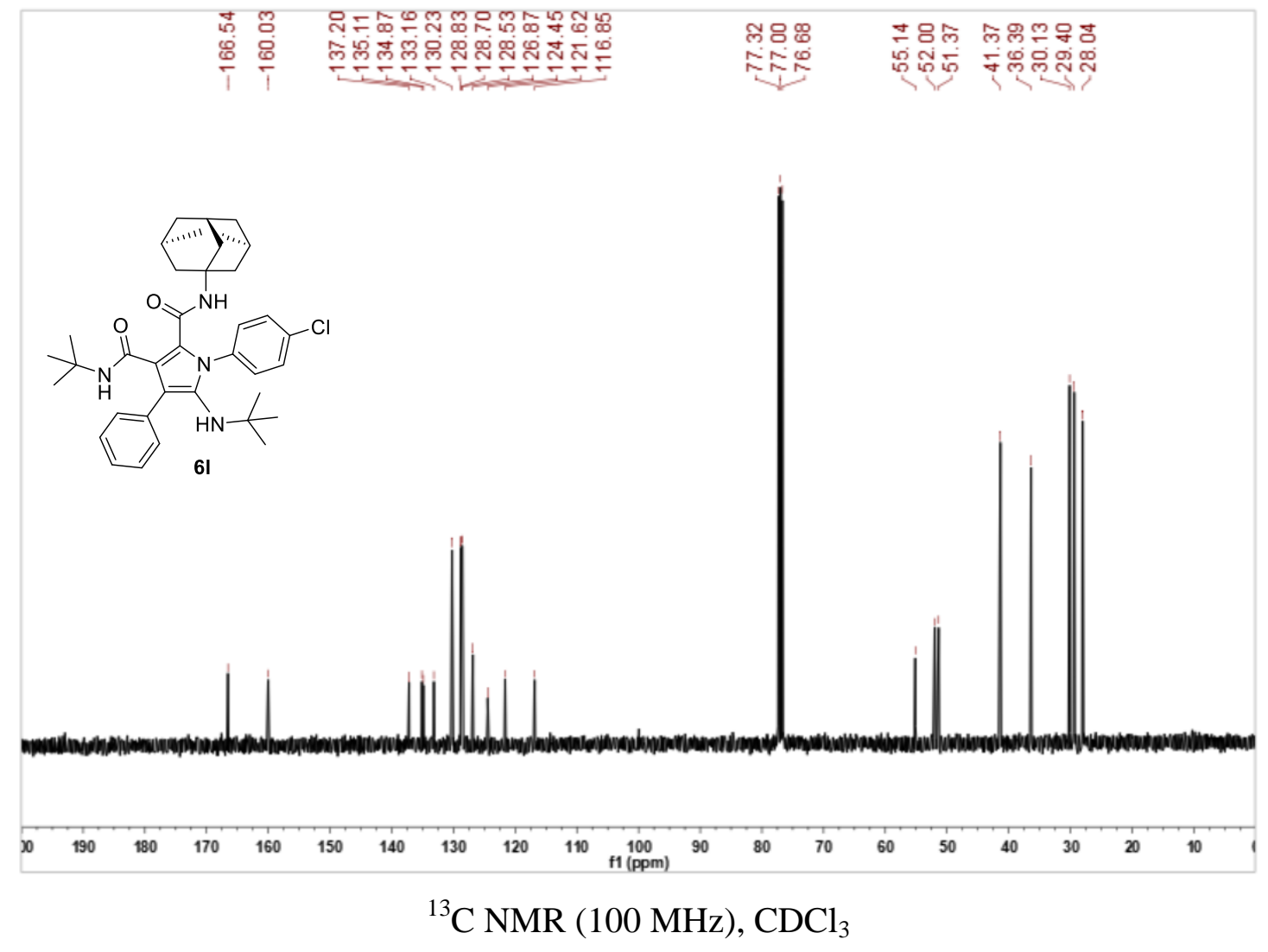




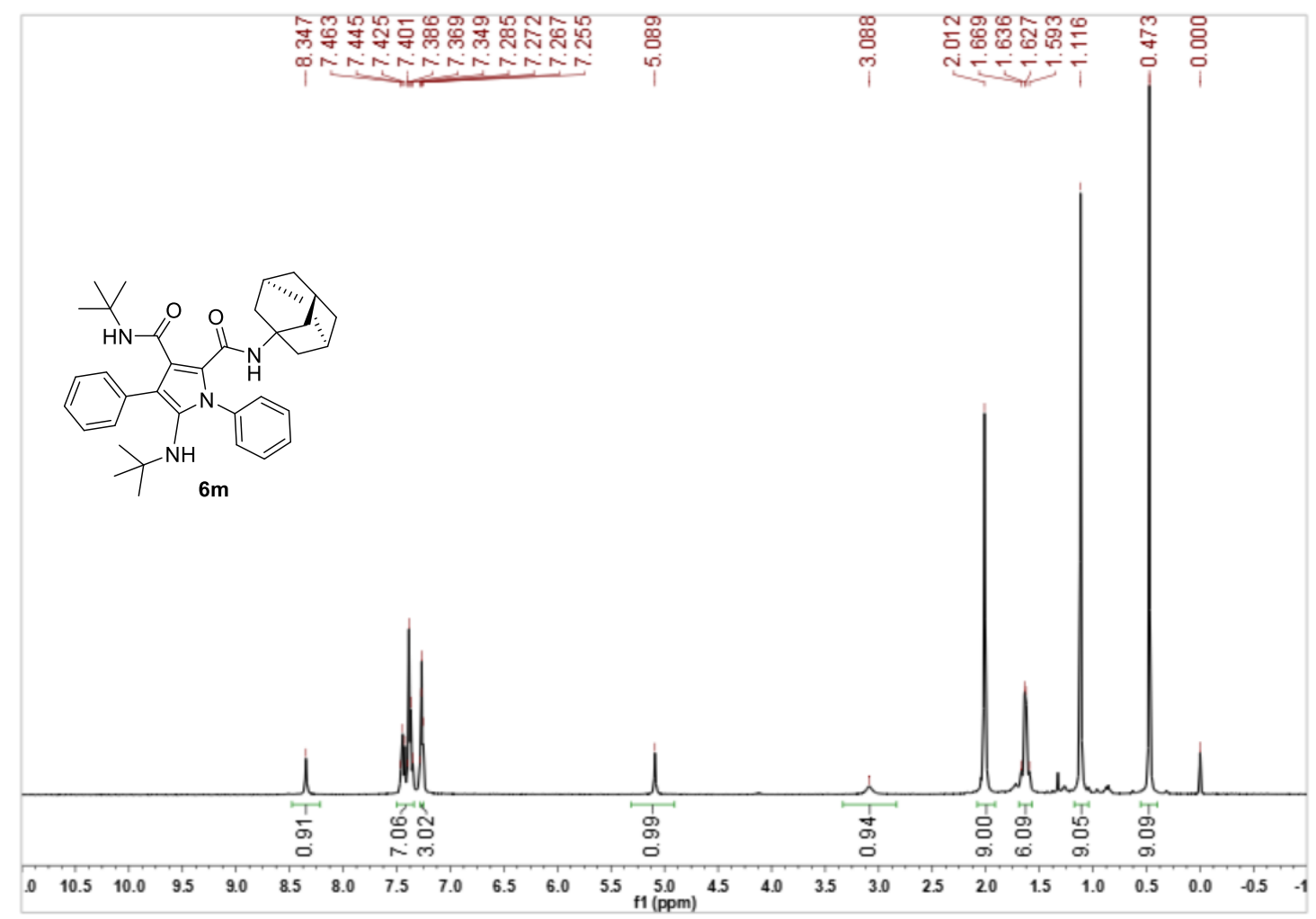

${ }^{1} \mathrm{H}$ NMR (400 MHz), $\mathrm{CDCl}_{3}$

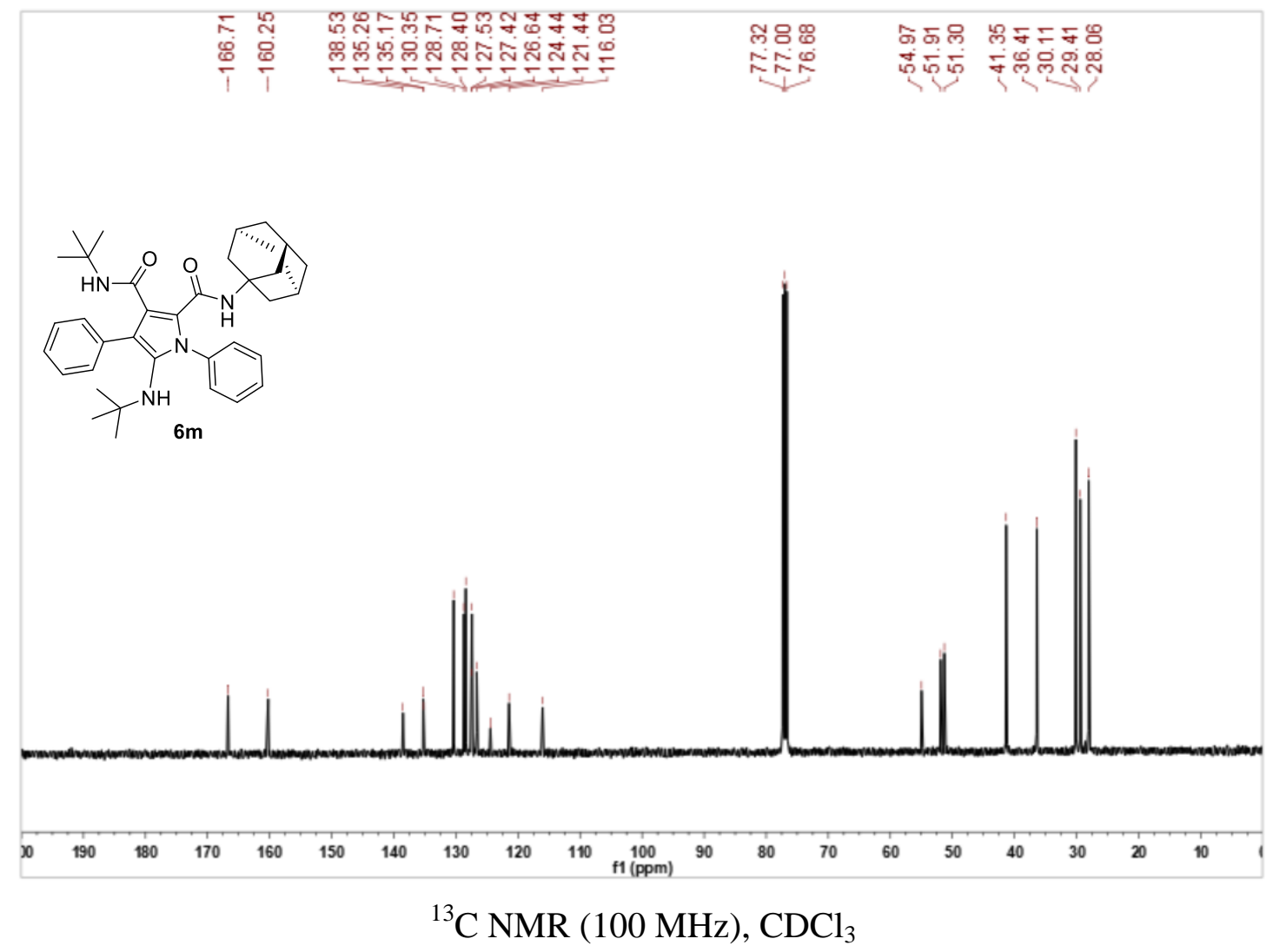




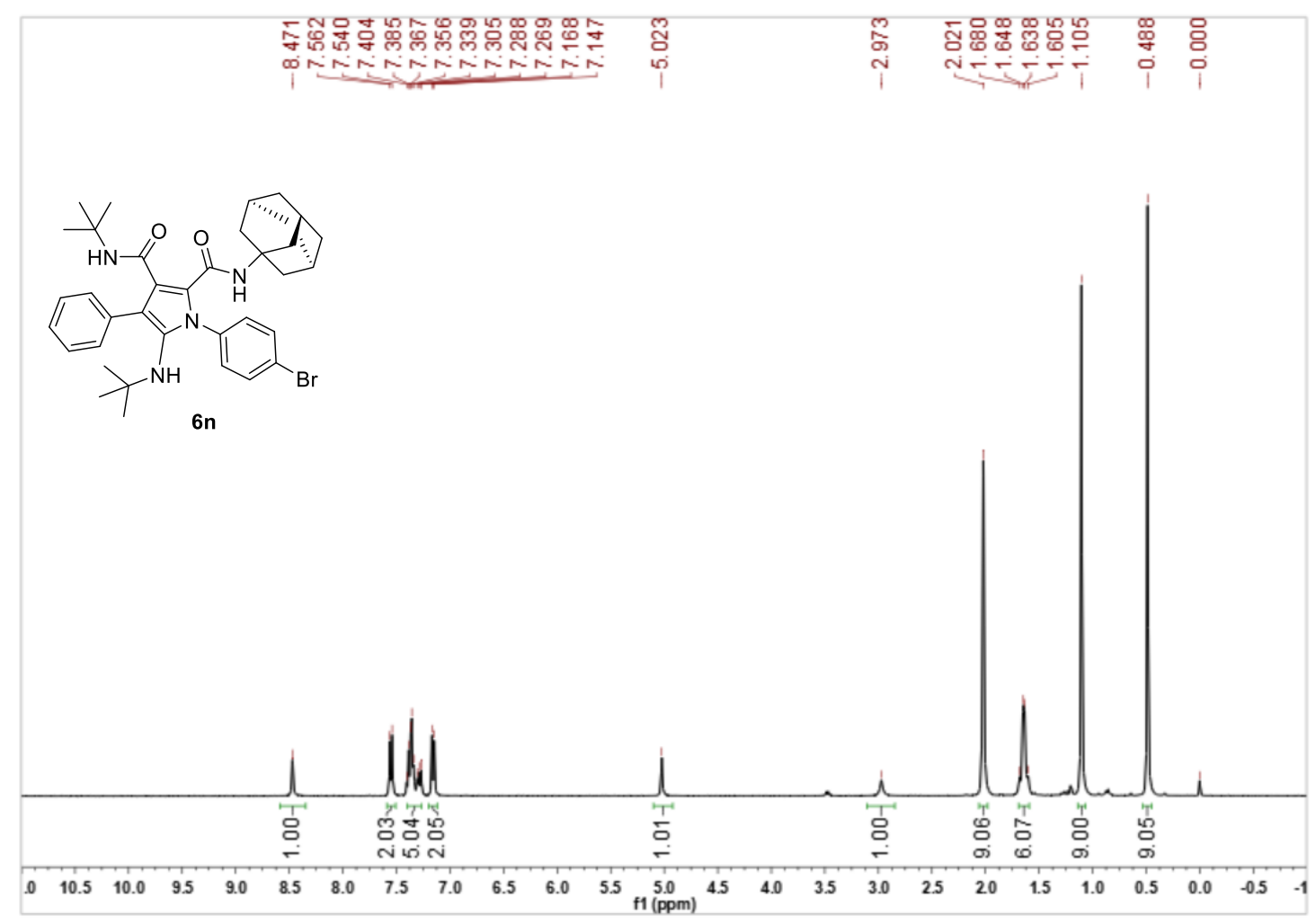

${ }^{1} \mathrm{H}$ NMR (400 MHz), $\mathrm{CDCl}_{3}$

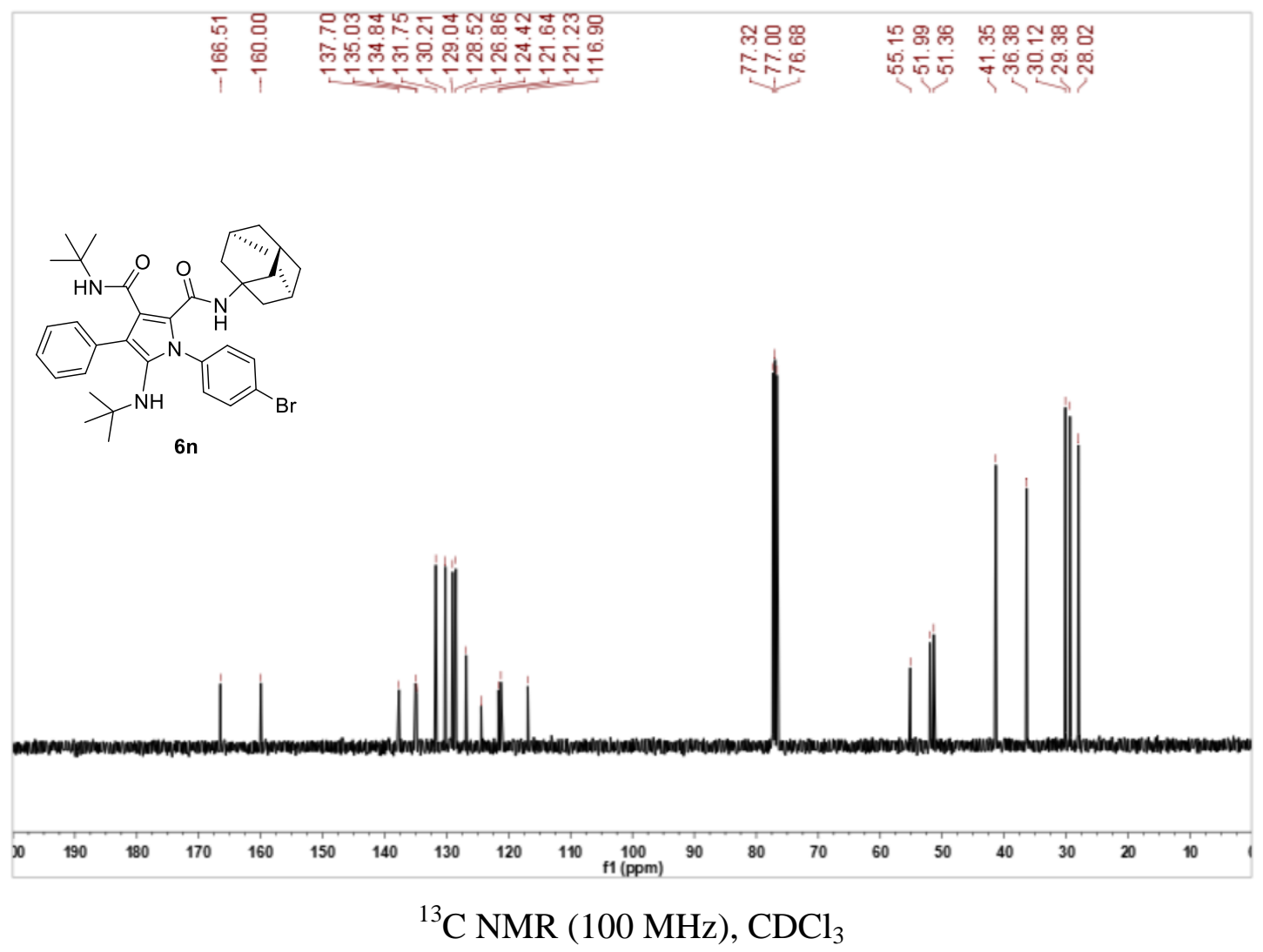




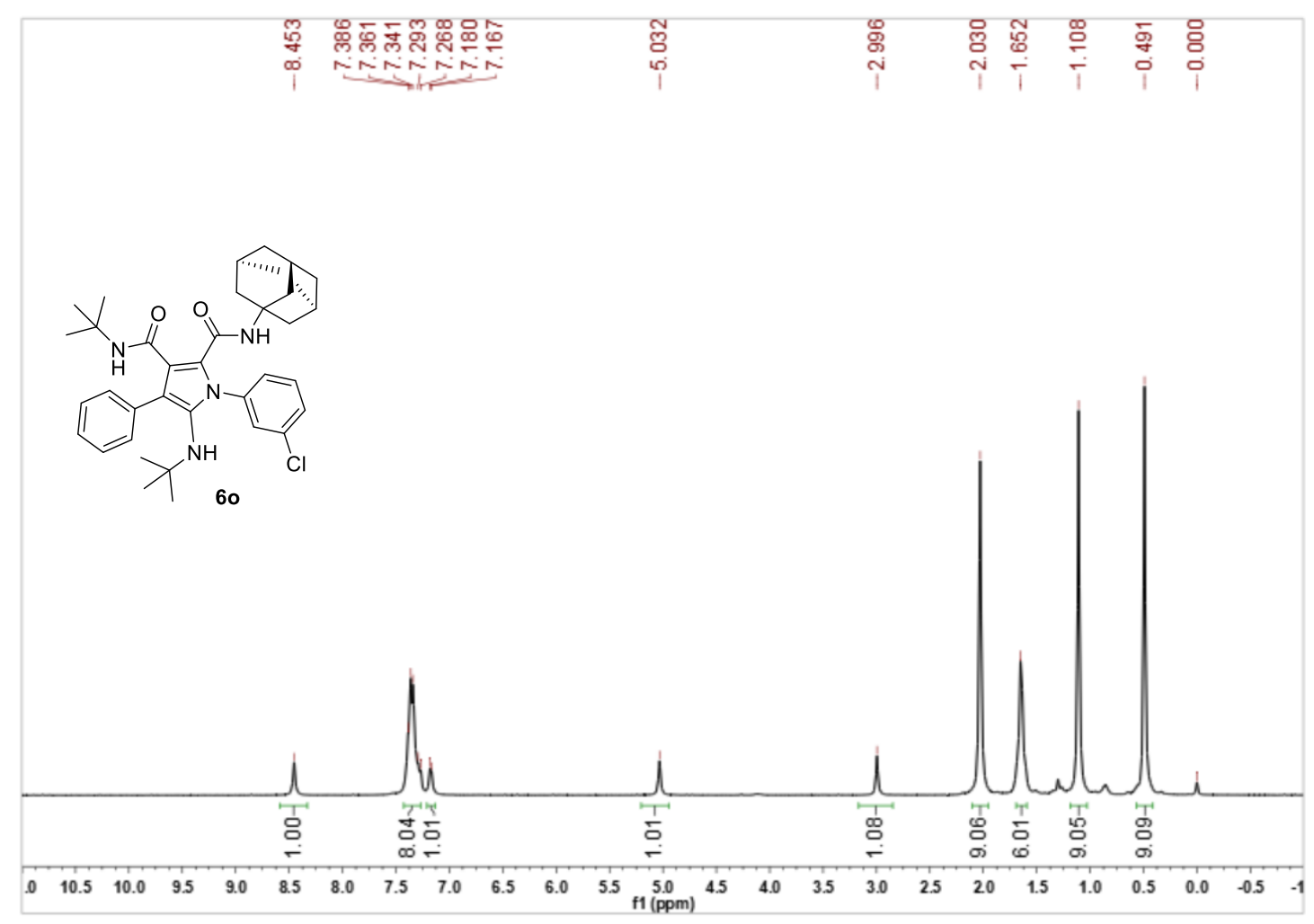

${ }^{1} \mathrm{H}$ NMR (400 MHz), $\mathrm{CDCl}_{3}$

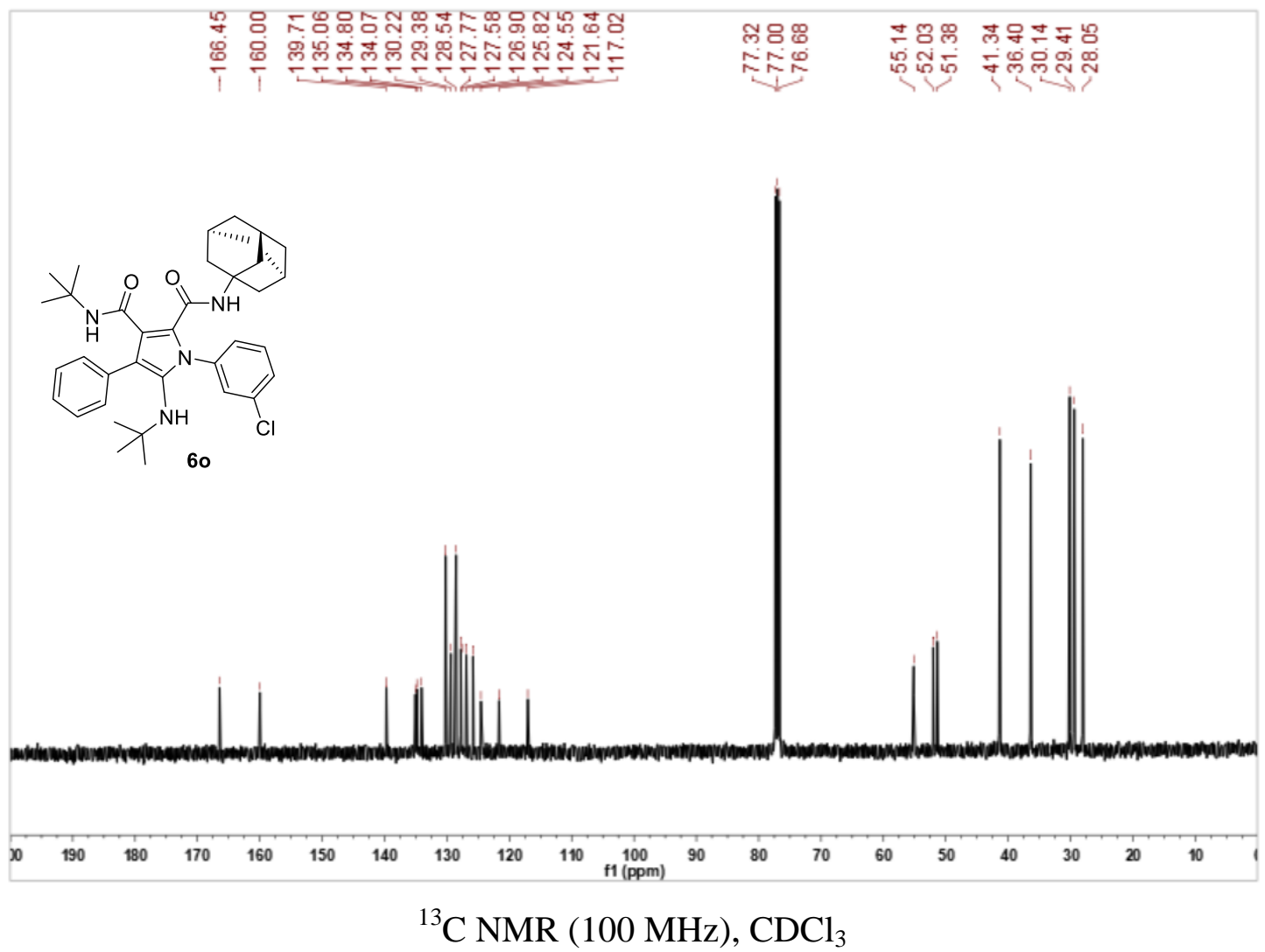




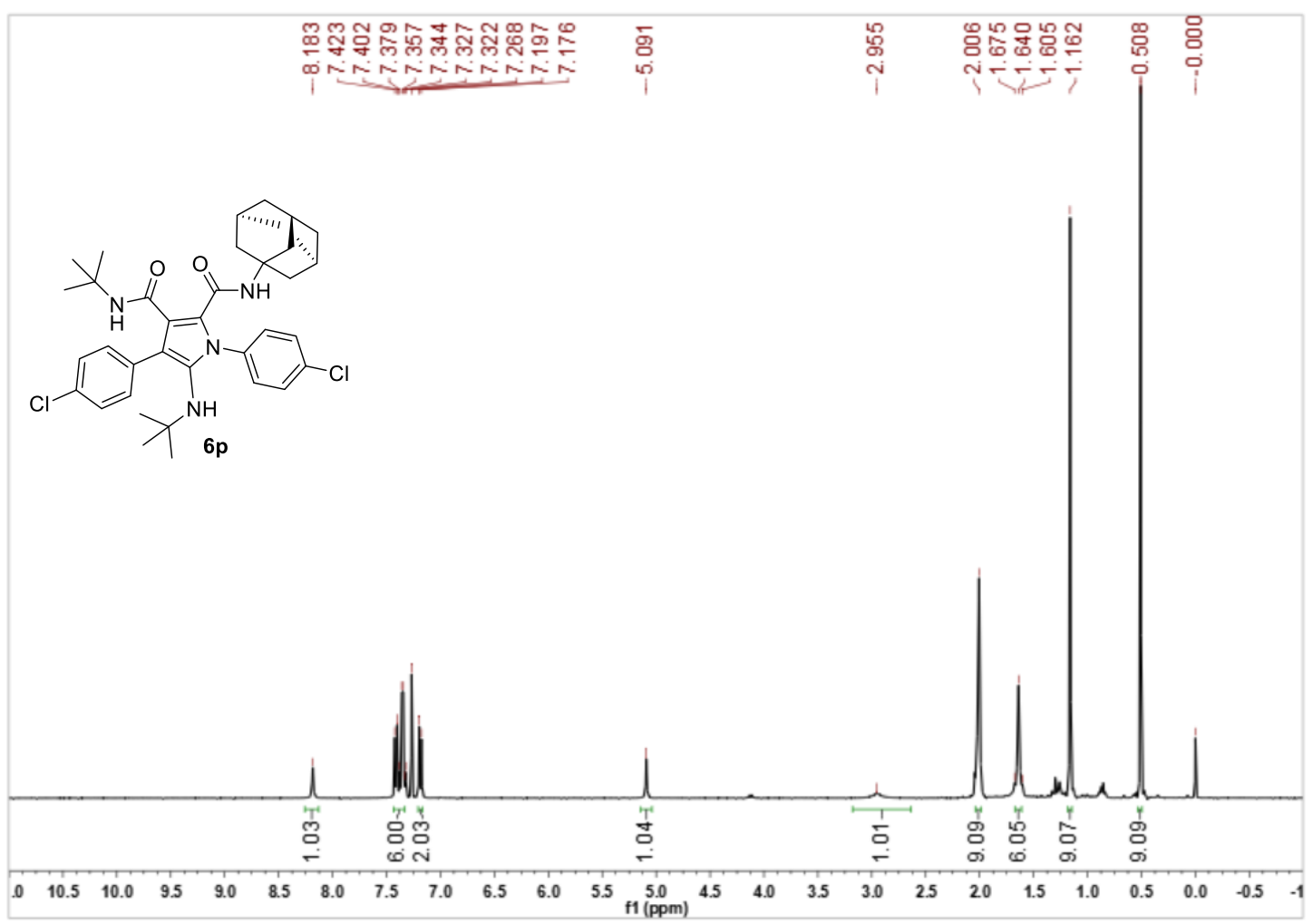

${ }^{1} \mathrm{H}$ NMR (400 MHz), $\mathrm{CDCl}_{3}$

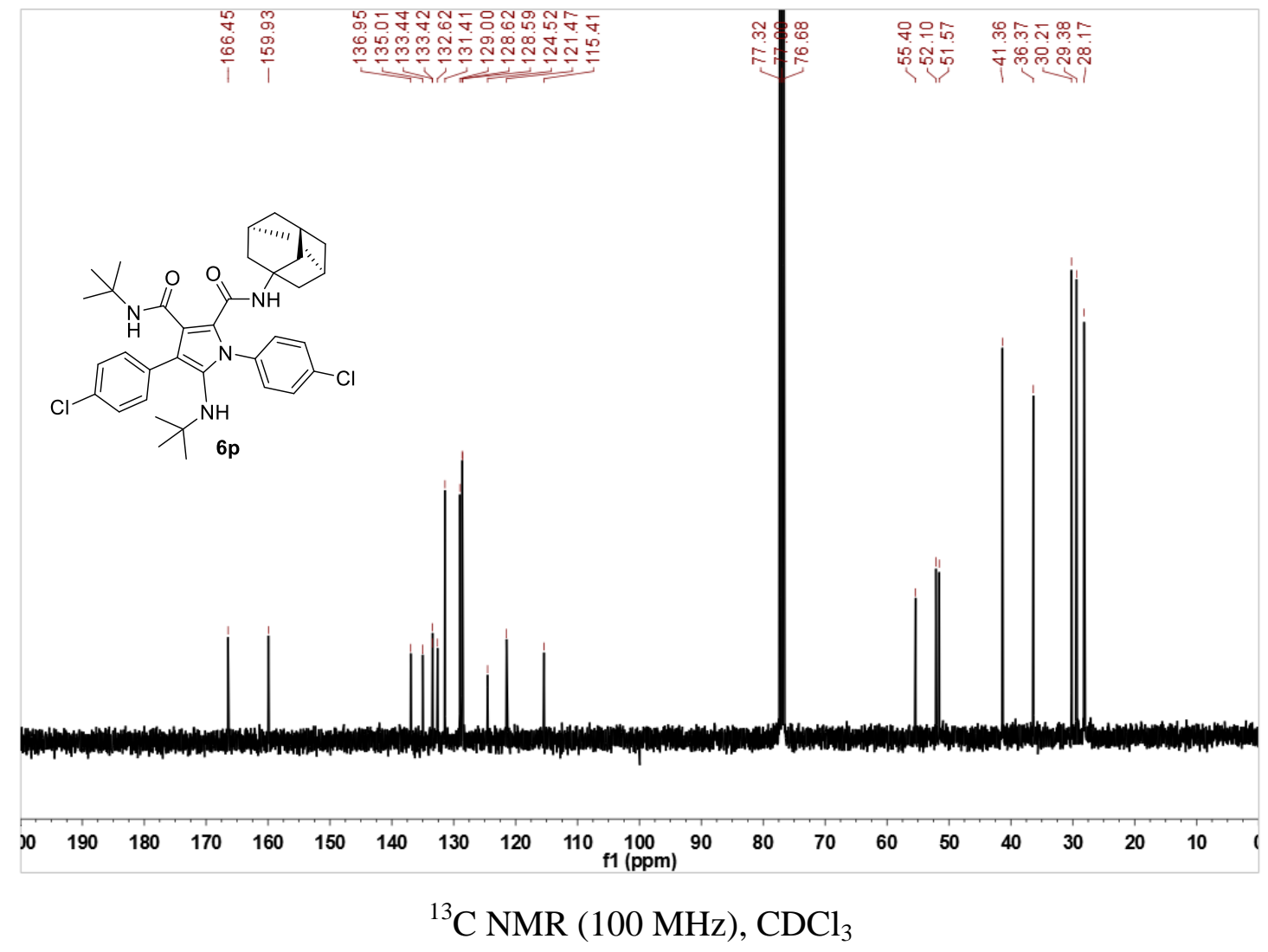




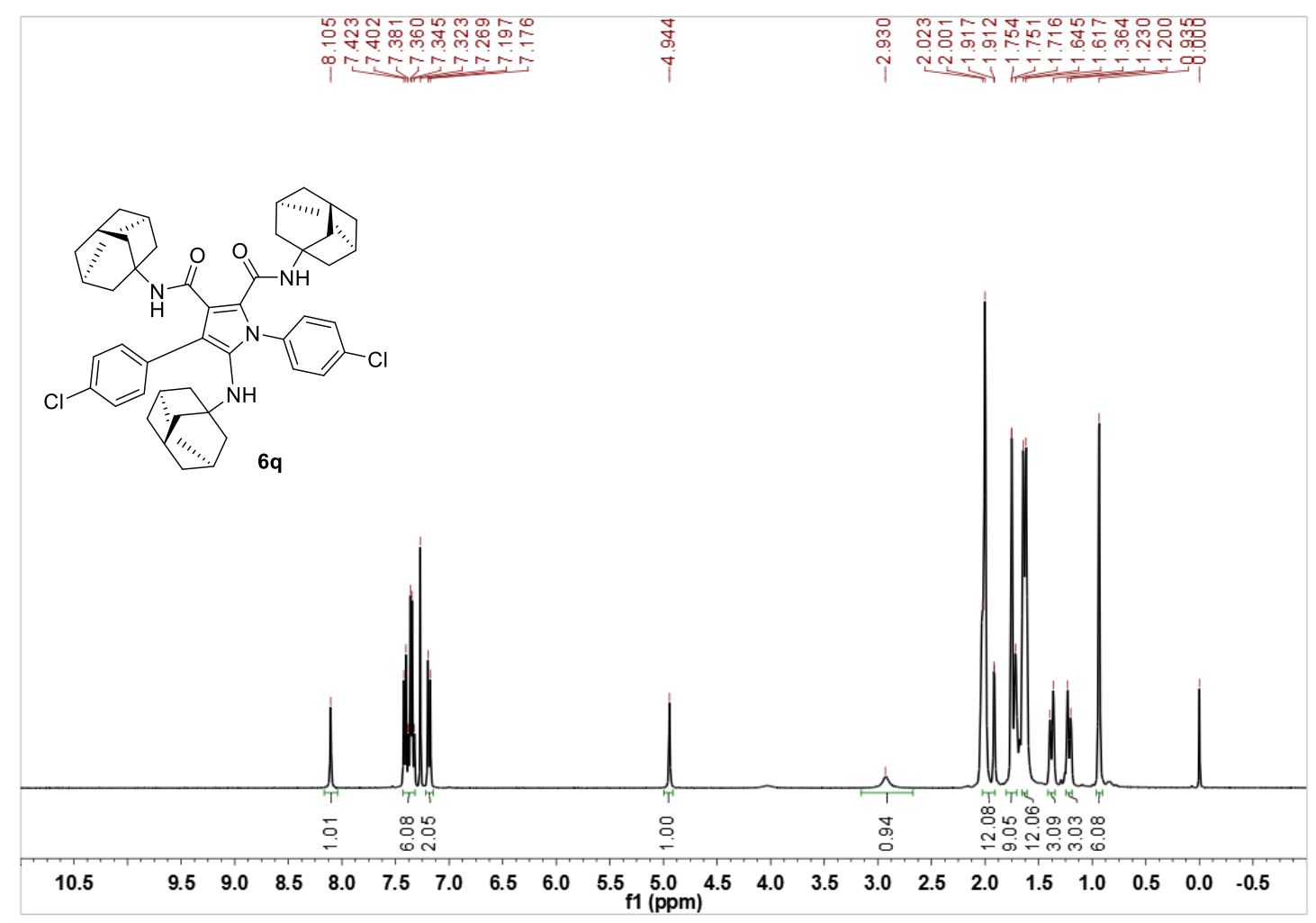

${ }^{1} \mathrm{H}$ NMR (400 MHz), $\mathrm{CDCl}_{3}$

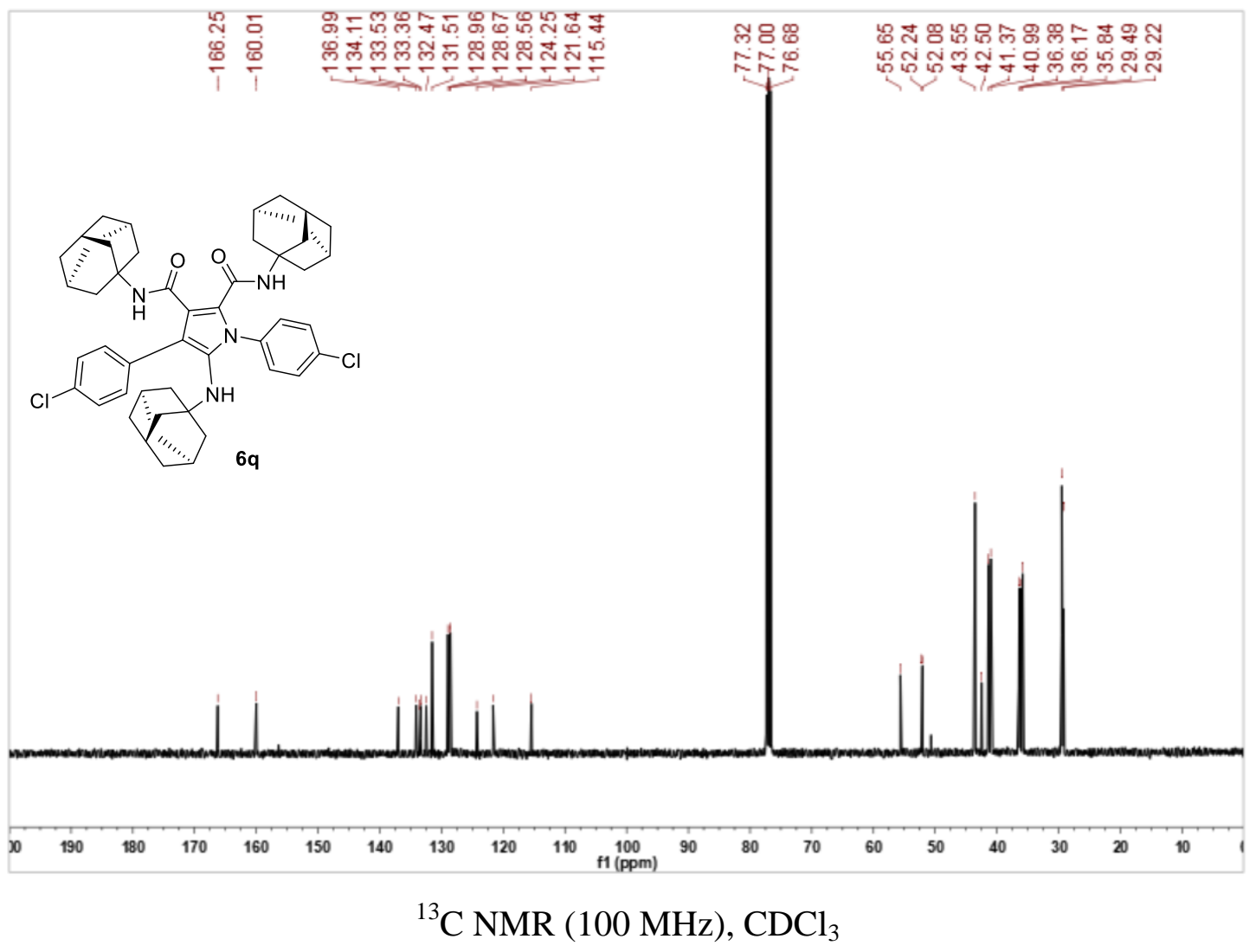




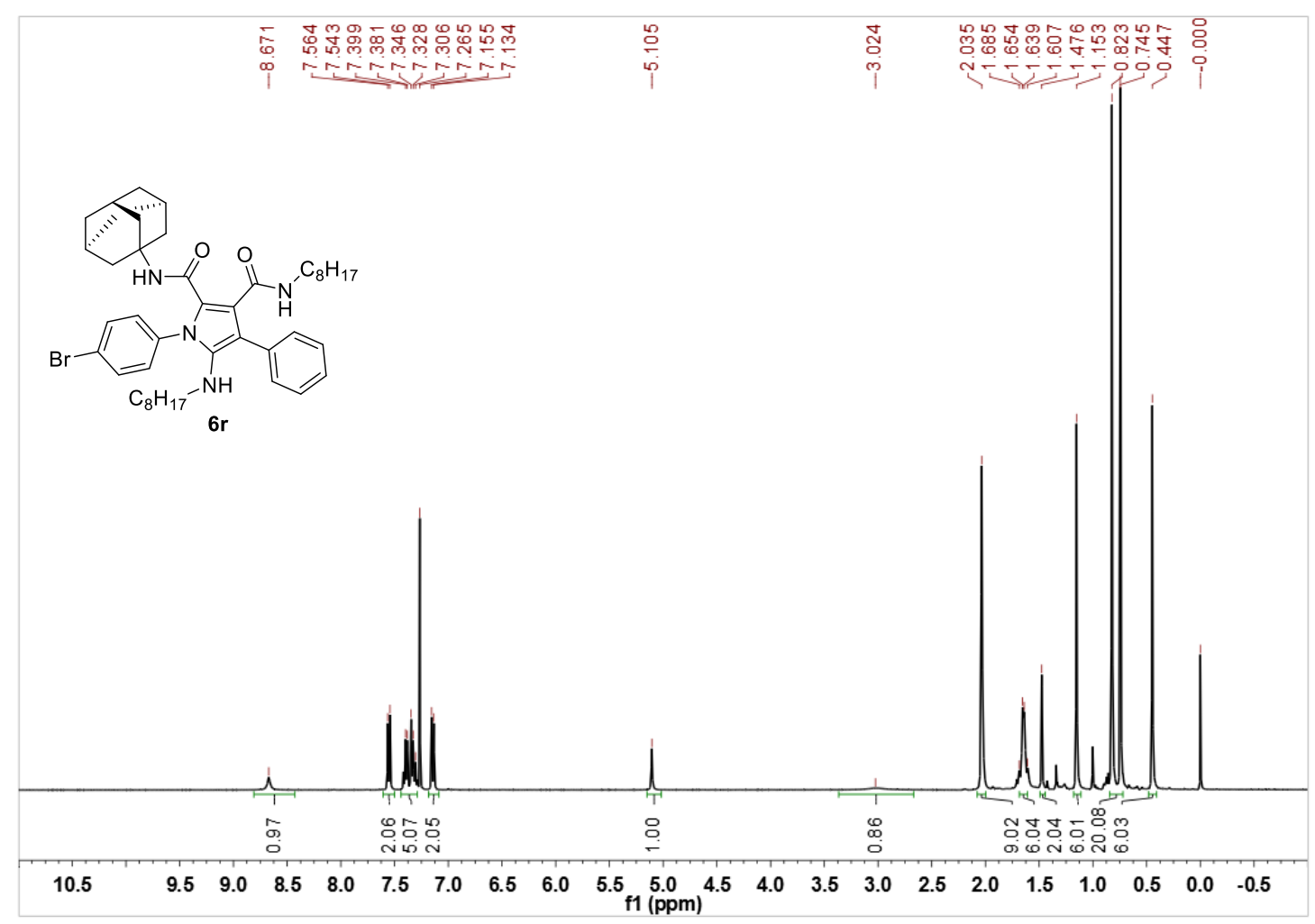

${ }^{1} \mathrm{H}$ NMR (400 MHz), $\mathrm{CDCl}_{3}$

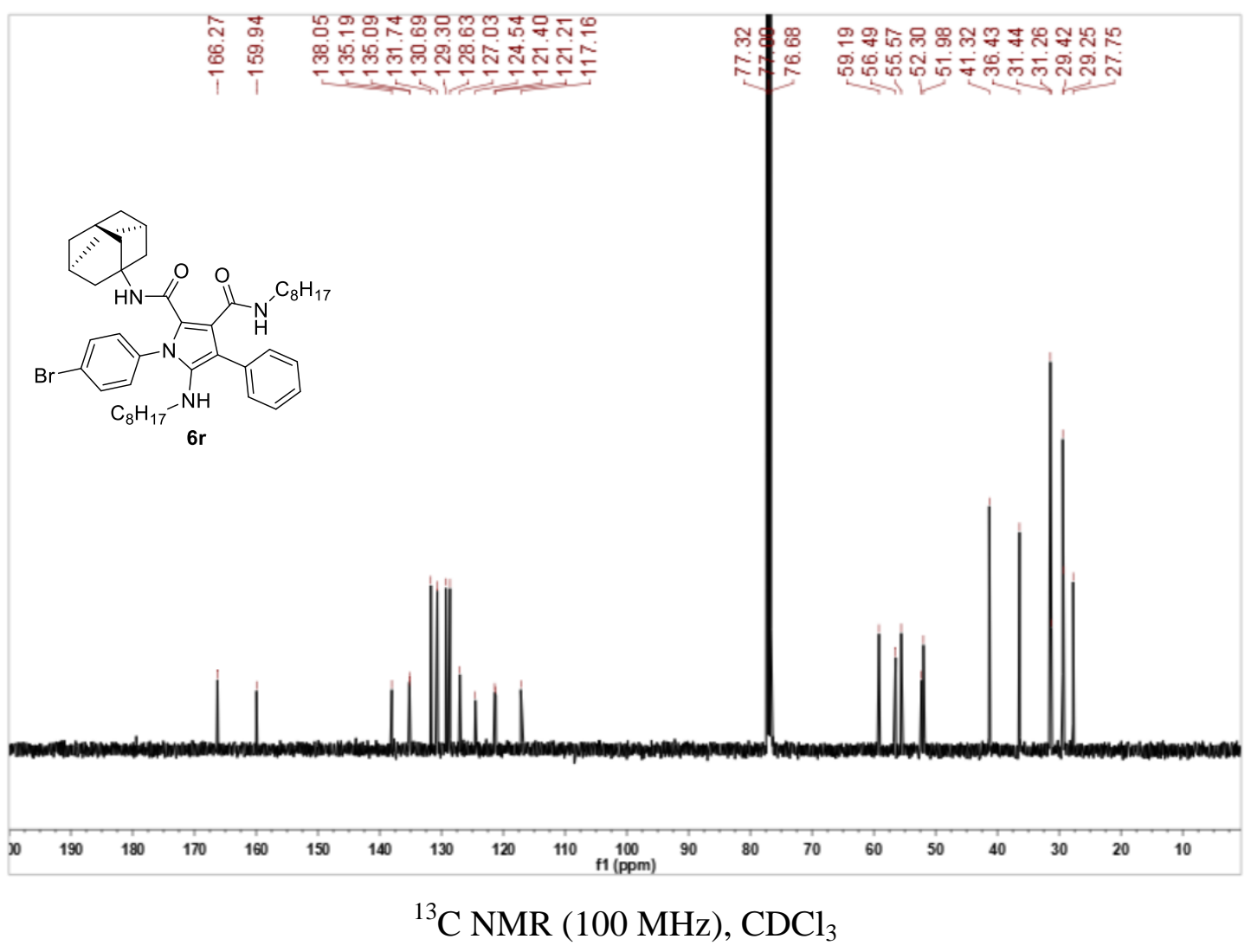




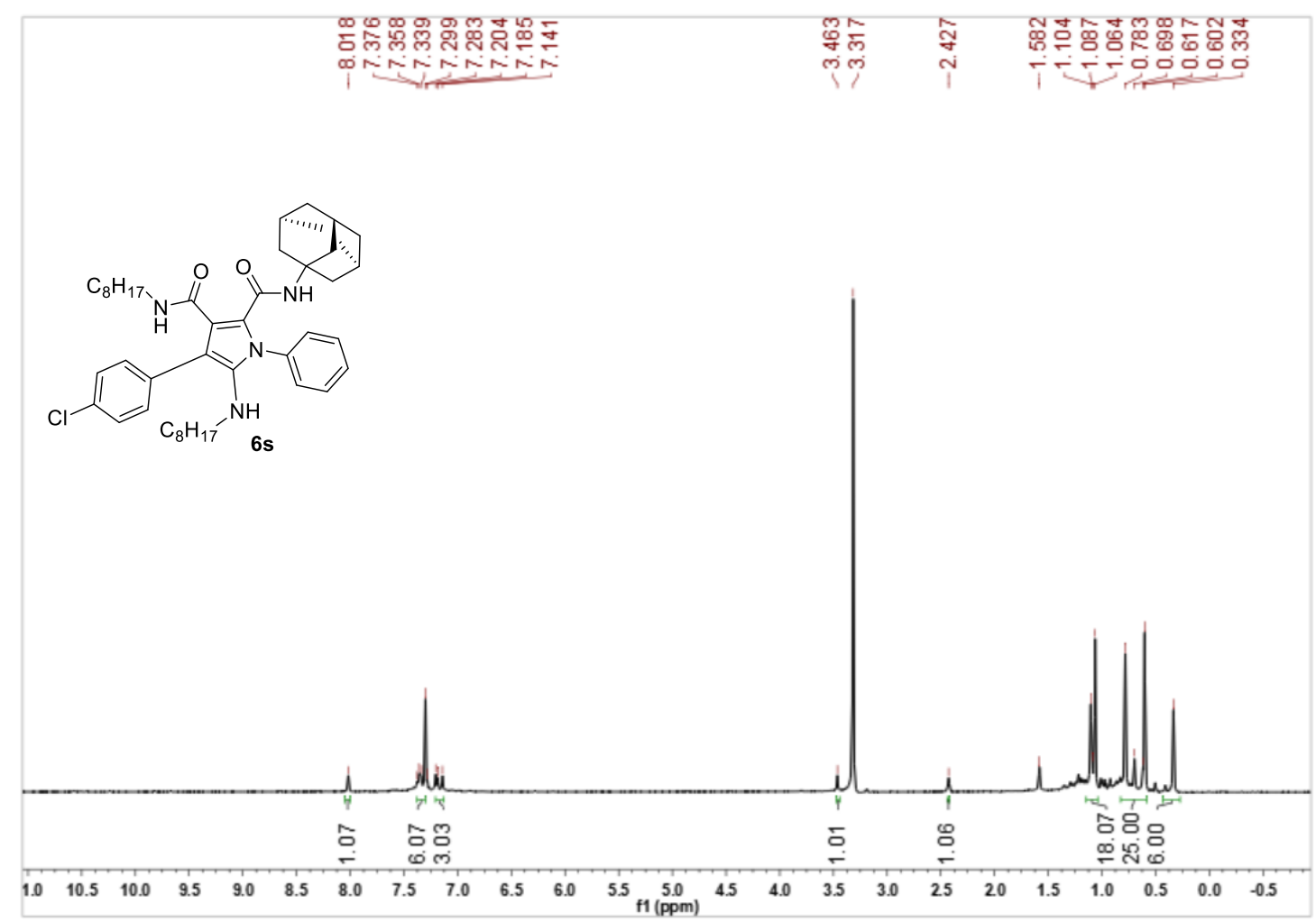

${ }^{1} \mathrm{H}$ NMR (400 MHz), DMSO- $d_{6}$

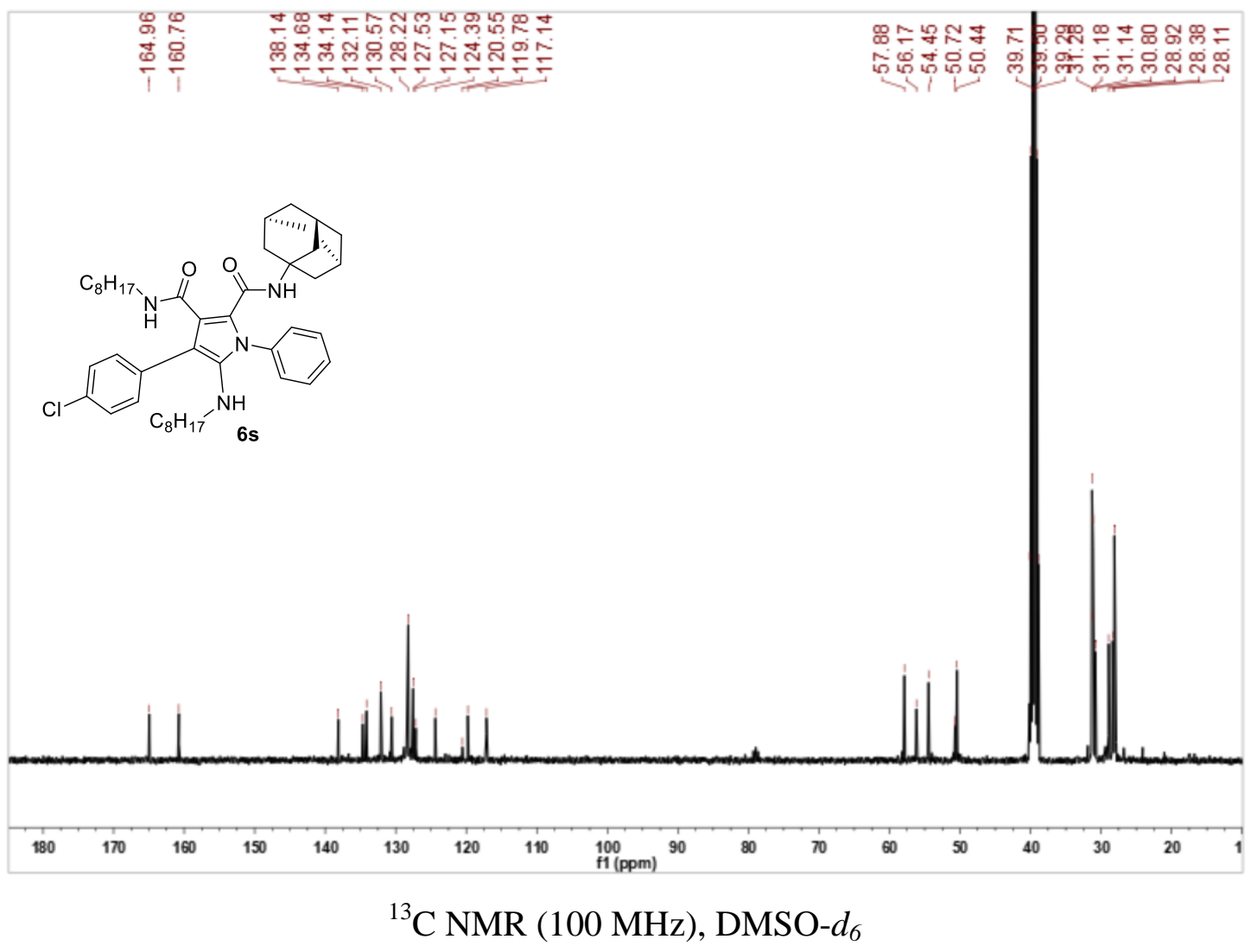




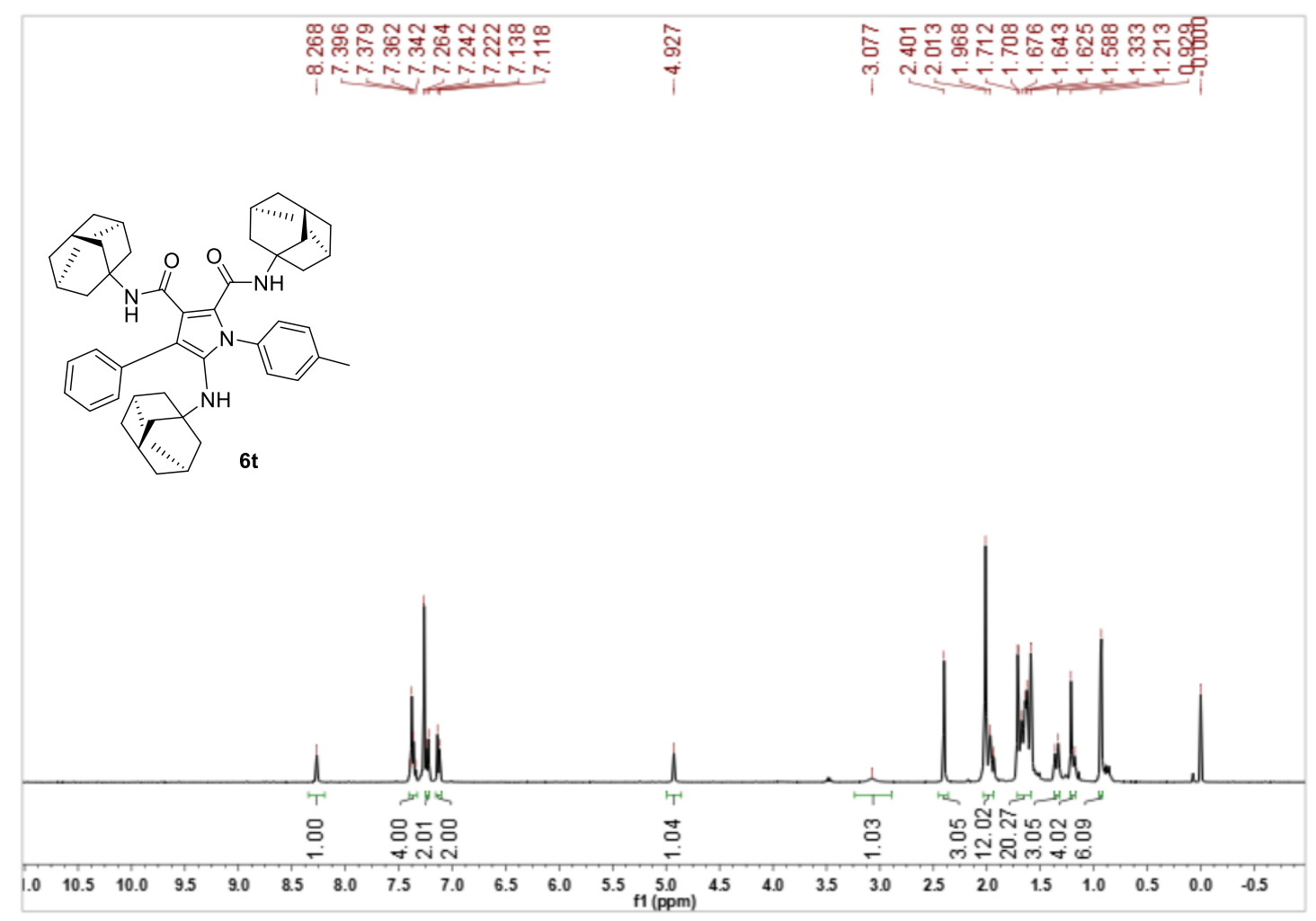

${ }^{1} \mathrm{H}$ NMR (400 MHz), $\mathrm{CDCl}_{3}$

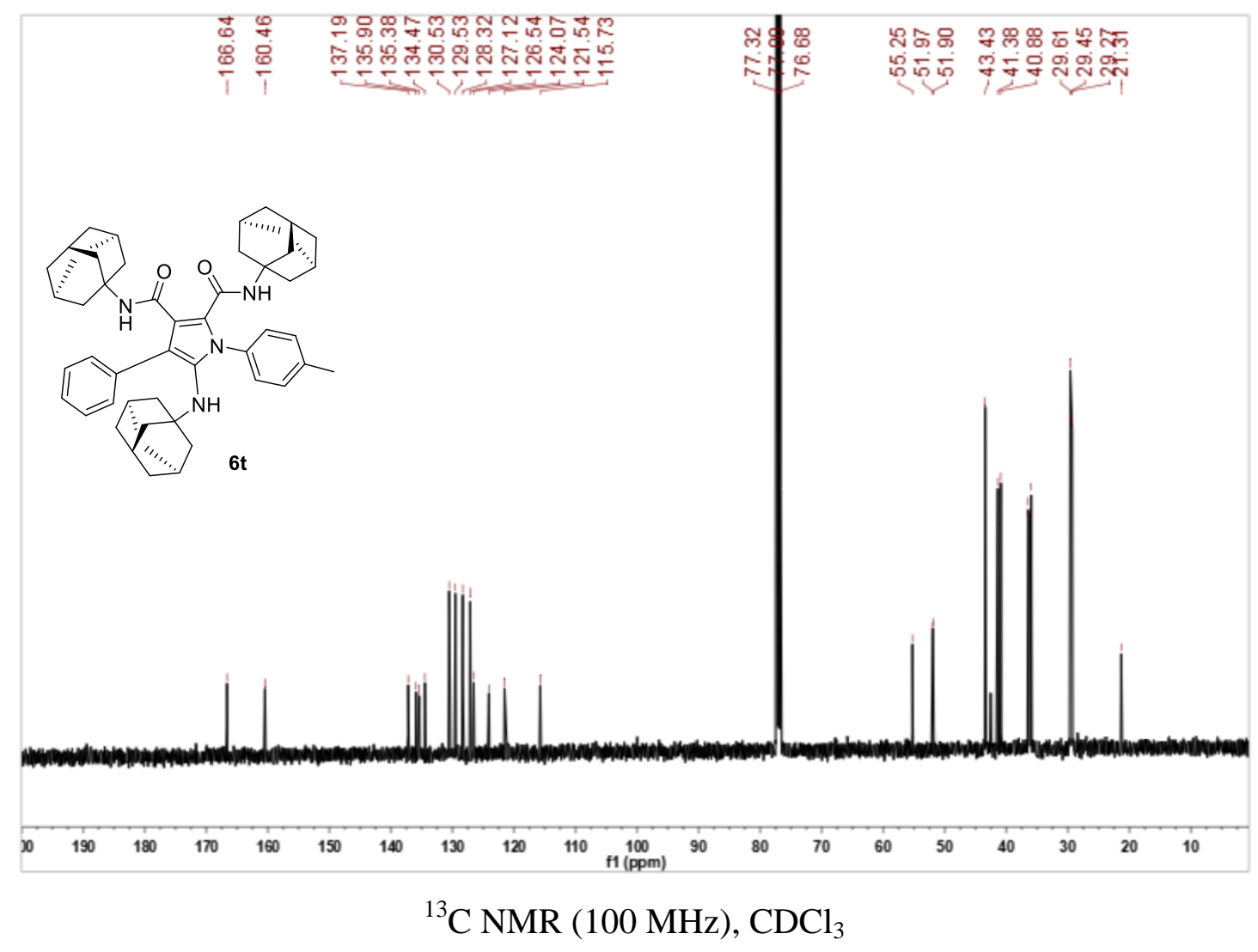




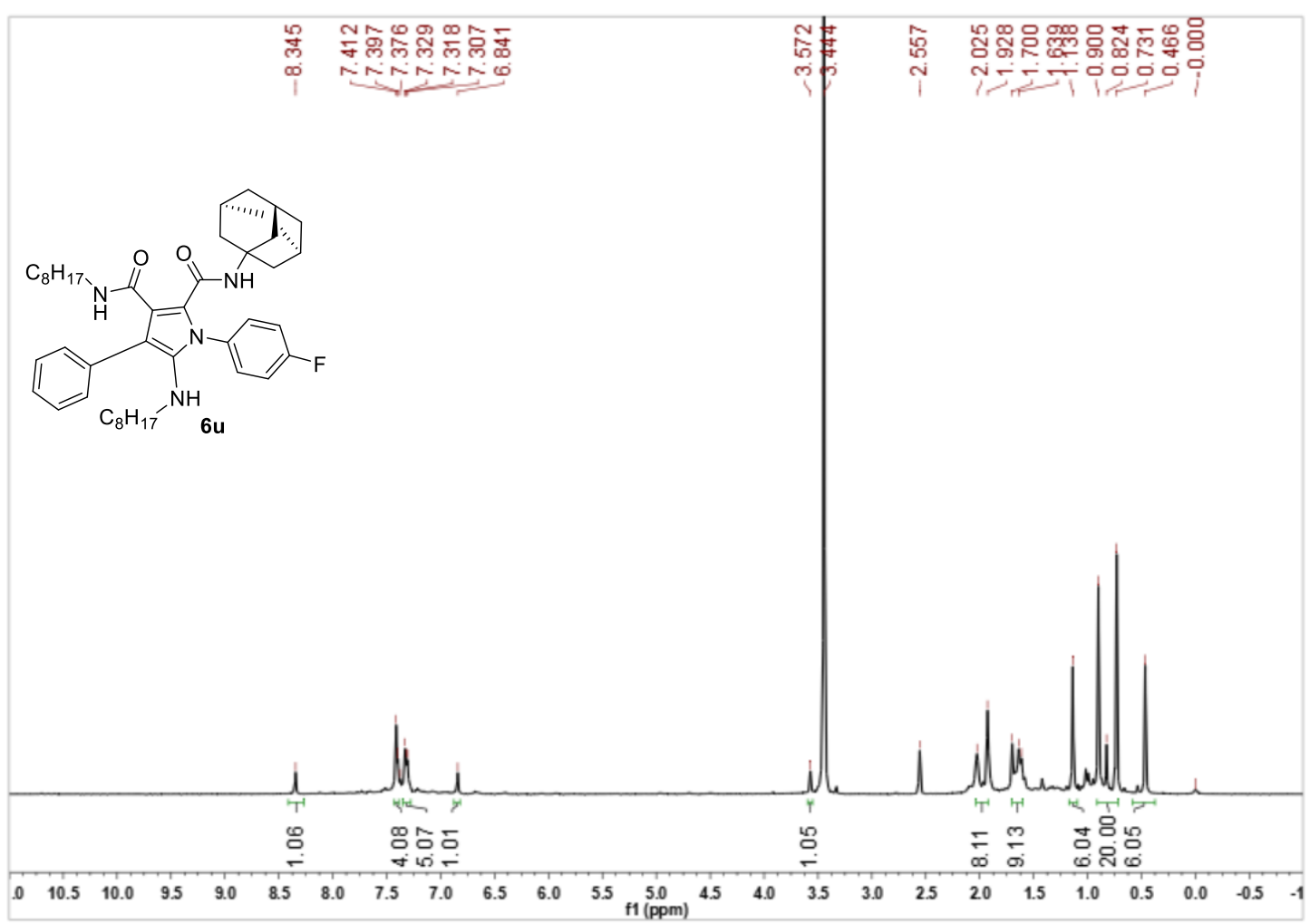

${ }^{1} \mathrm{H}$ NMR (400 MHz), DMSO- $d_{6}$

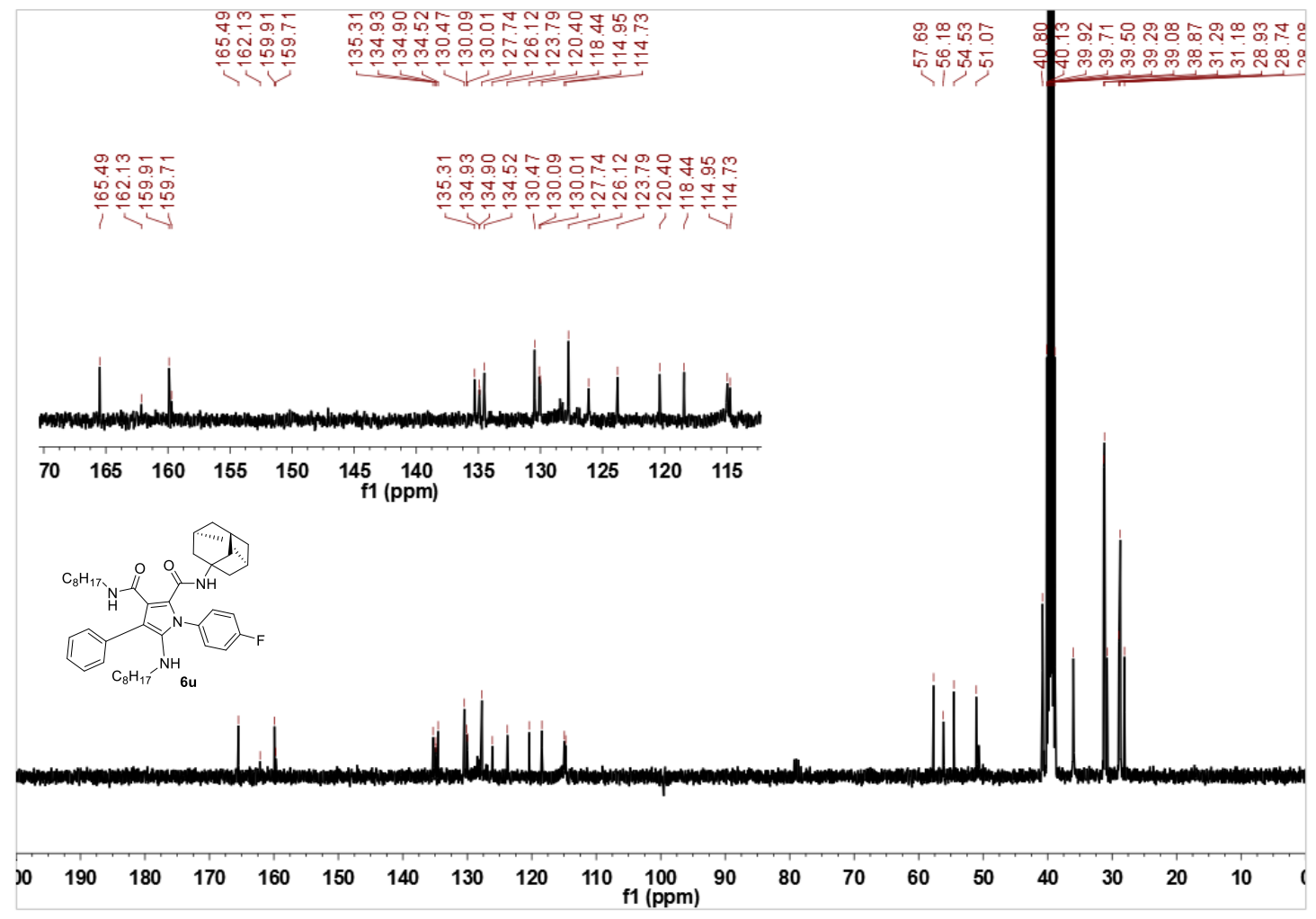

${ }^{13} \mathrm{C}$ NMR (100 MHz), DMSO- $d_{6}$ 


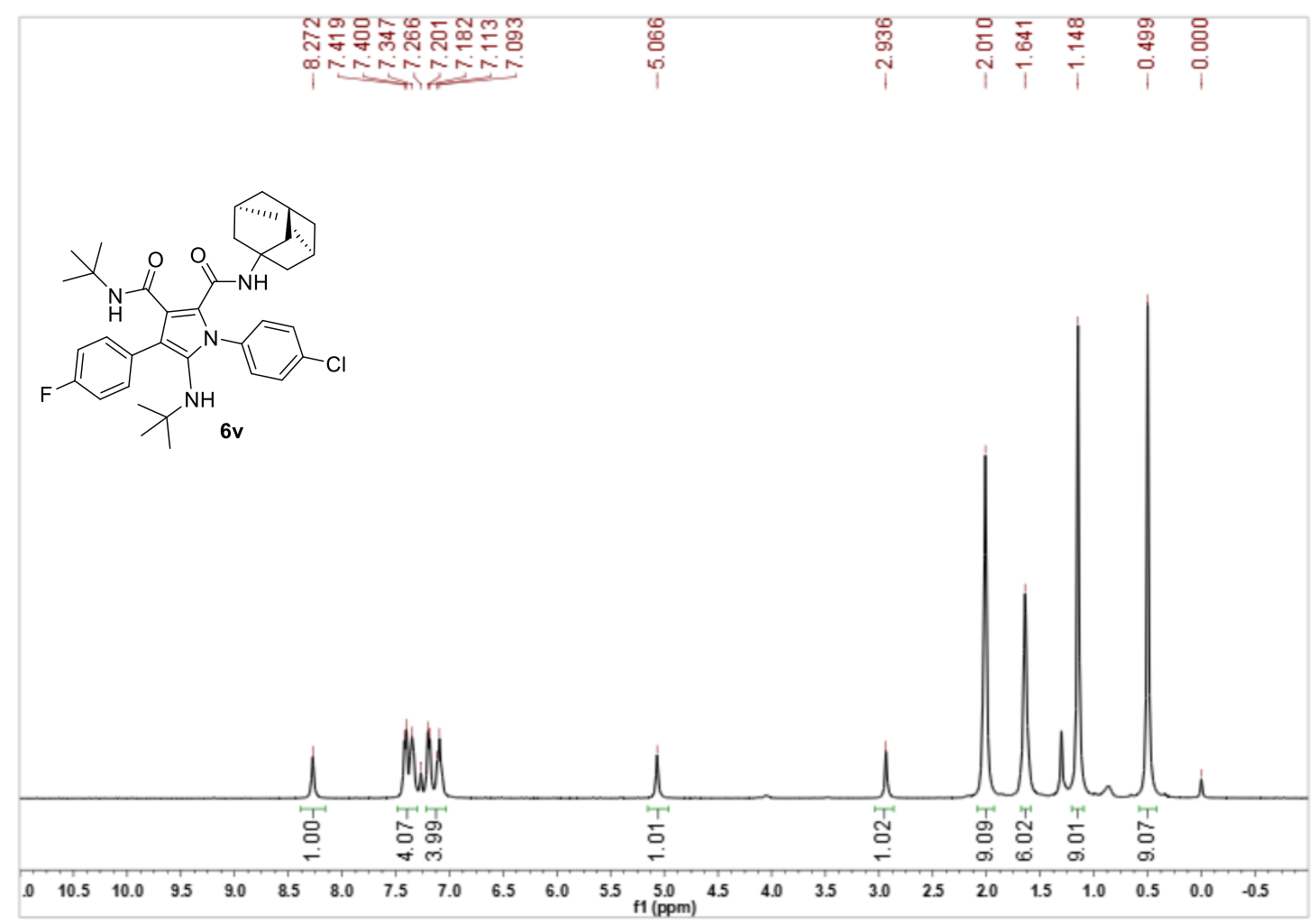

${ }^{1} \mathrm{H}$ NMR (400 MHz), $\mathrm{CDCl}_{3}$

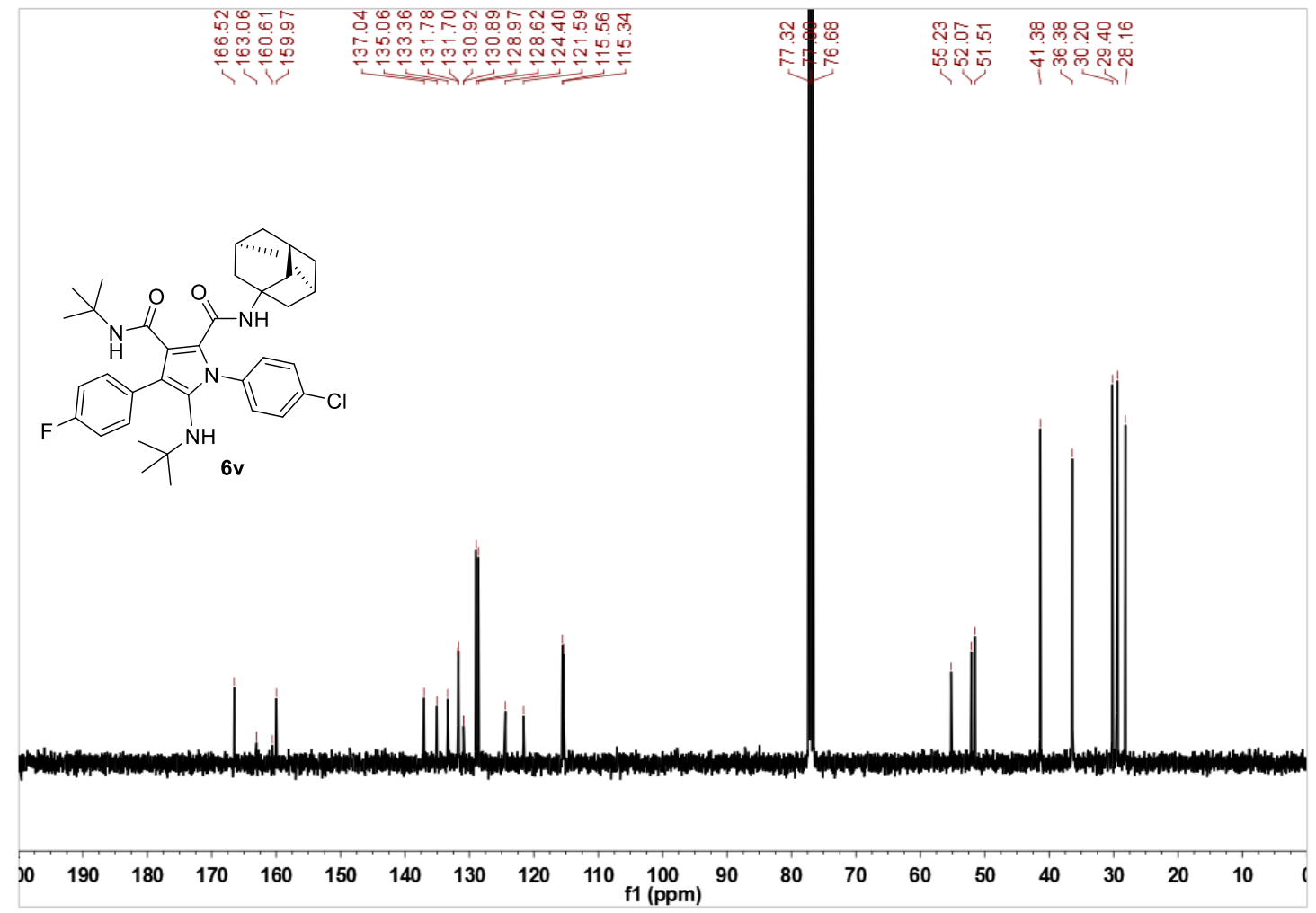

${ }^{13} \mathrm{C}$ NMR (100 MHz), $\mathrm{CDCl}_{3}$ 


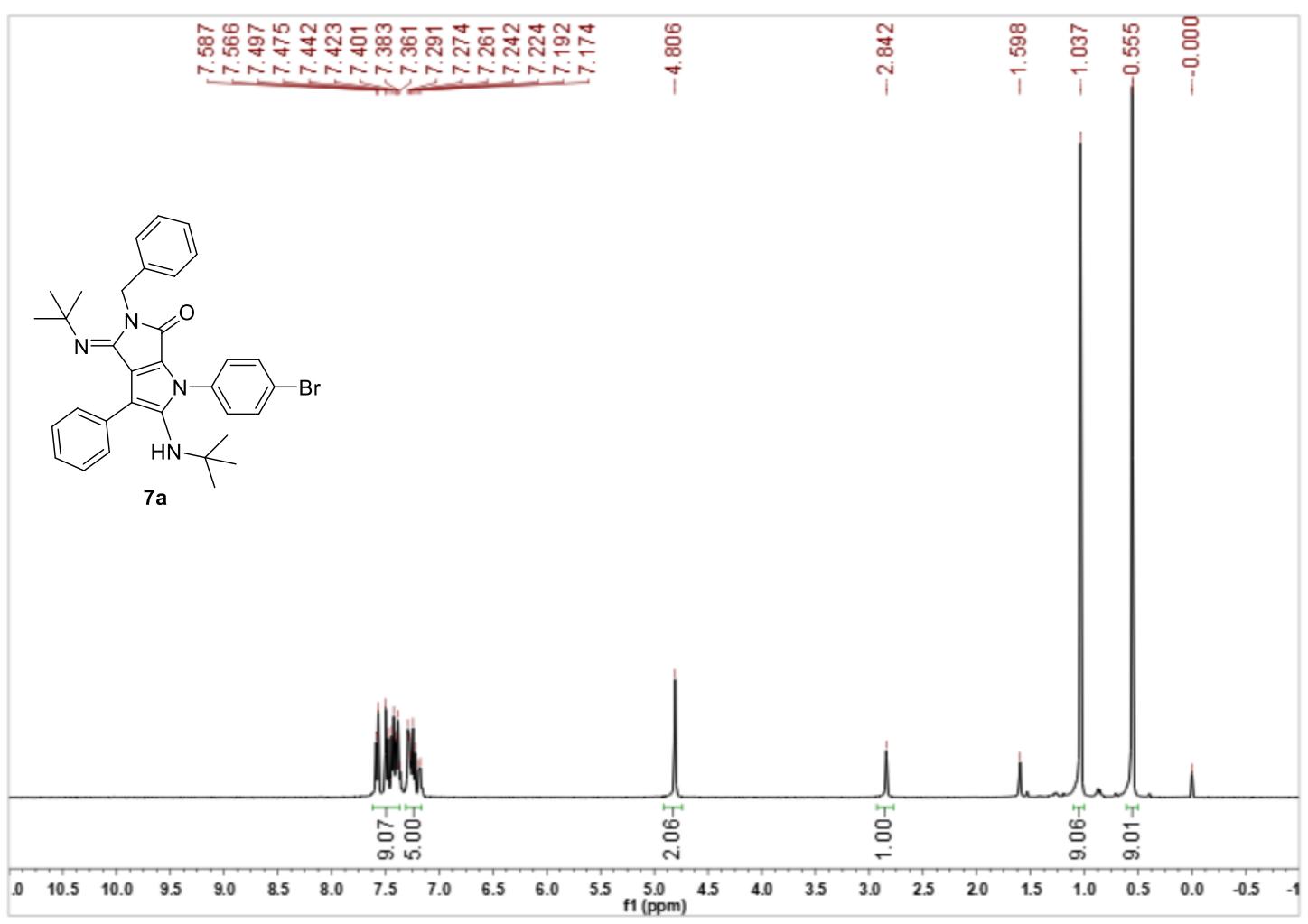

${ }^{1} \mathrm{H}$ NMR $(400 \mathrm{MHz}), \mathrm{CDCl}_{3}$

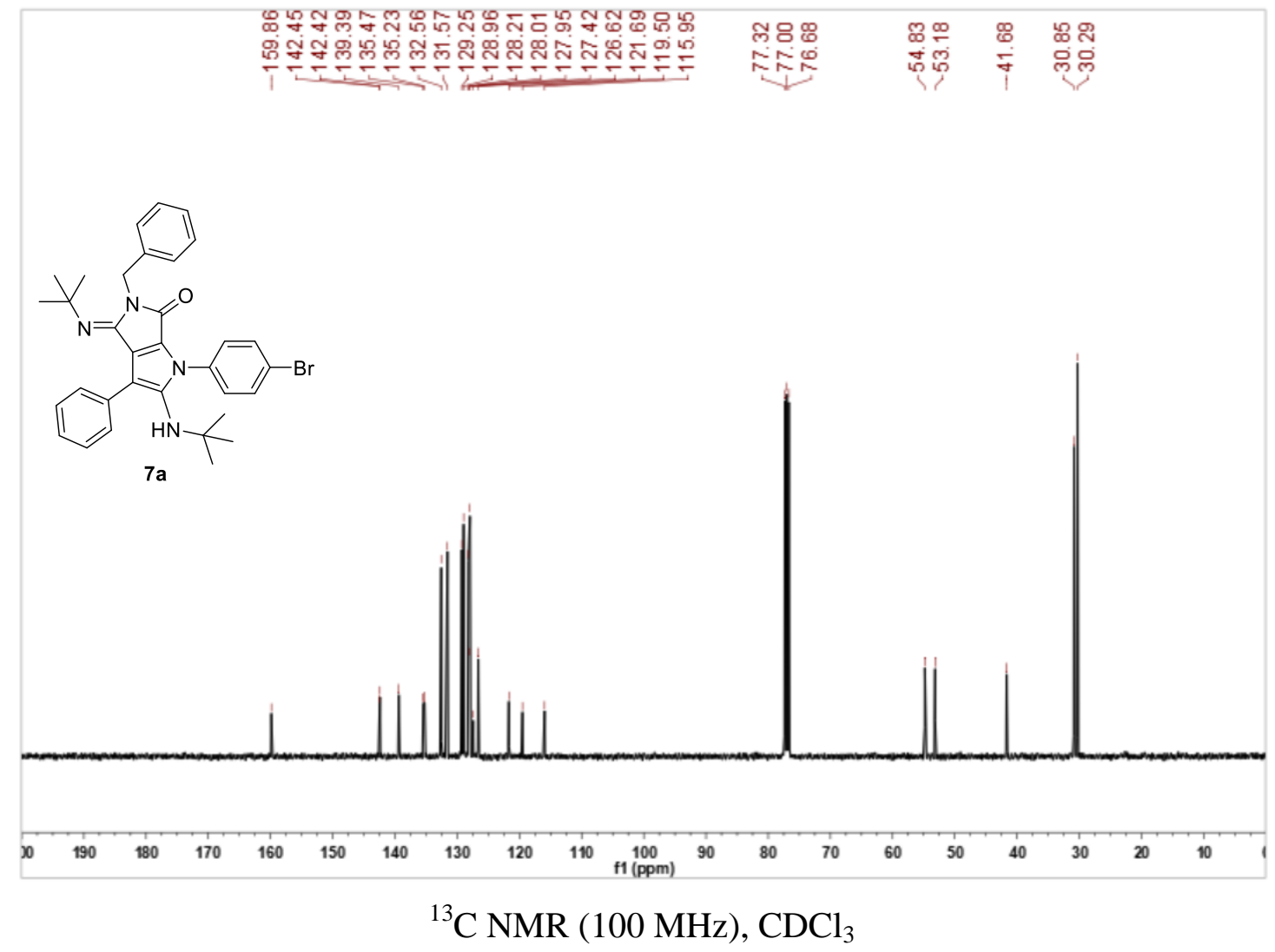




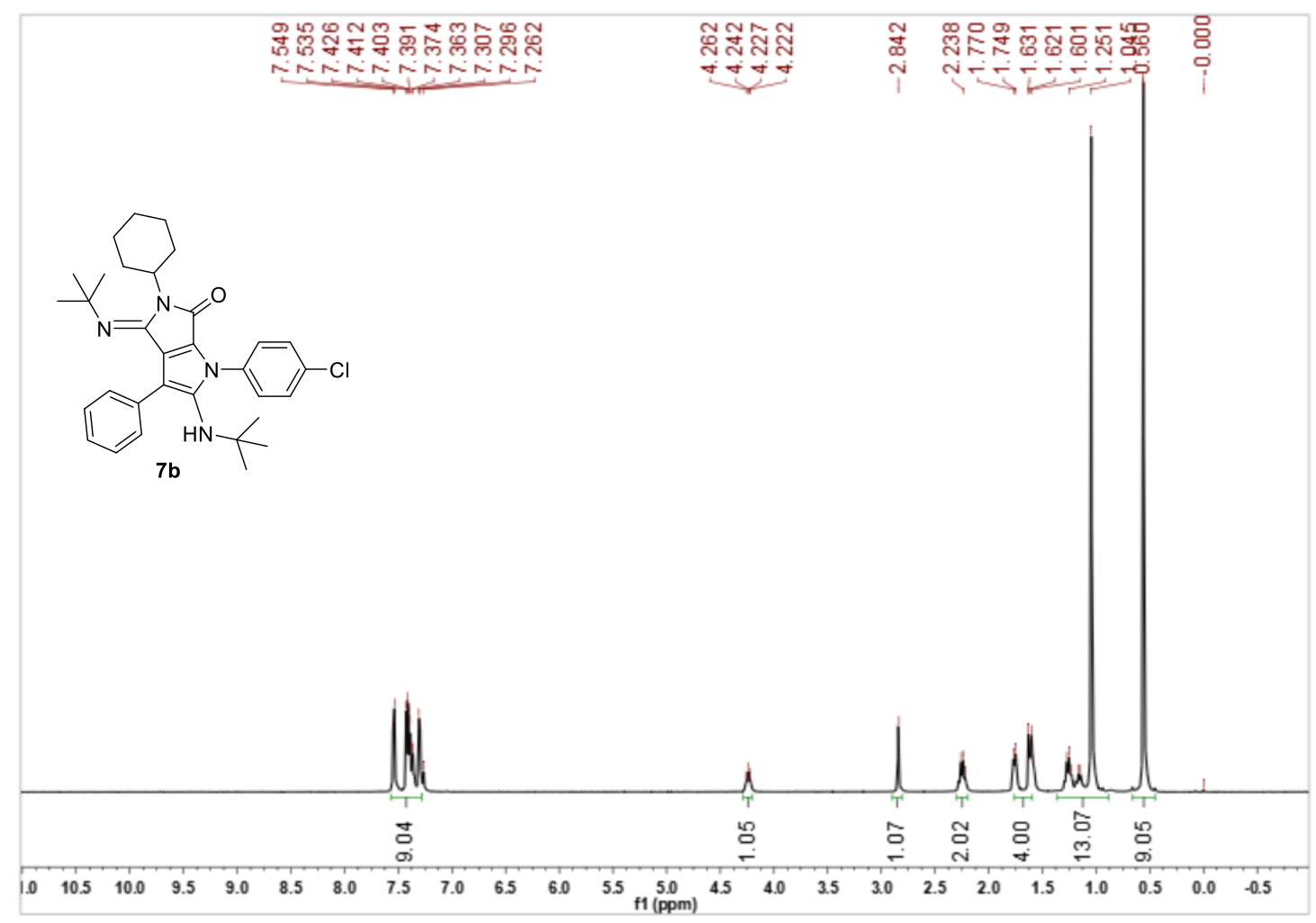

${ }^{1} \mathrm{H}$ NMR (400 MHz), $\mathrm{CDCl}_{3}$

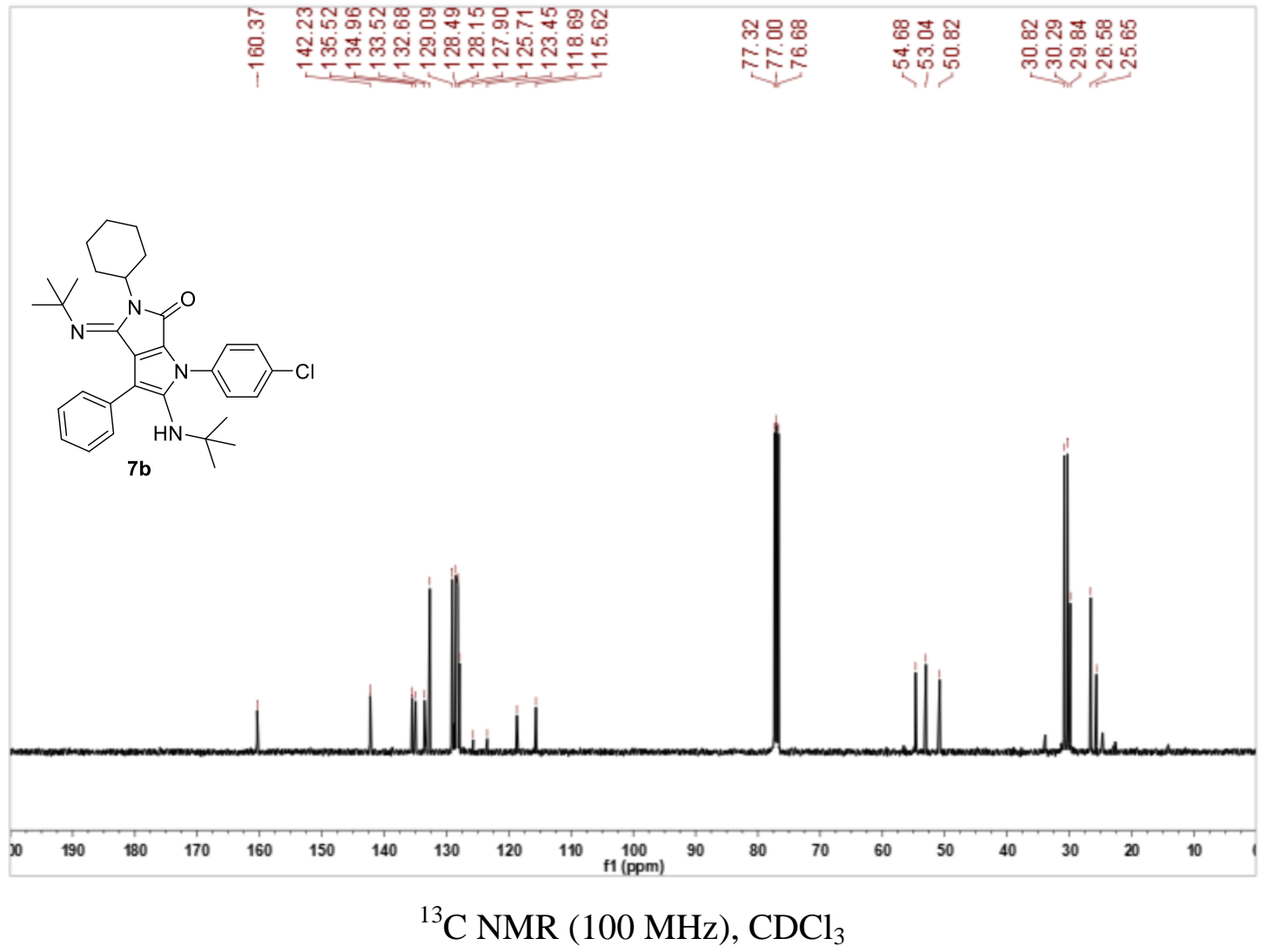




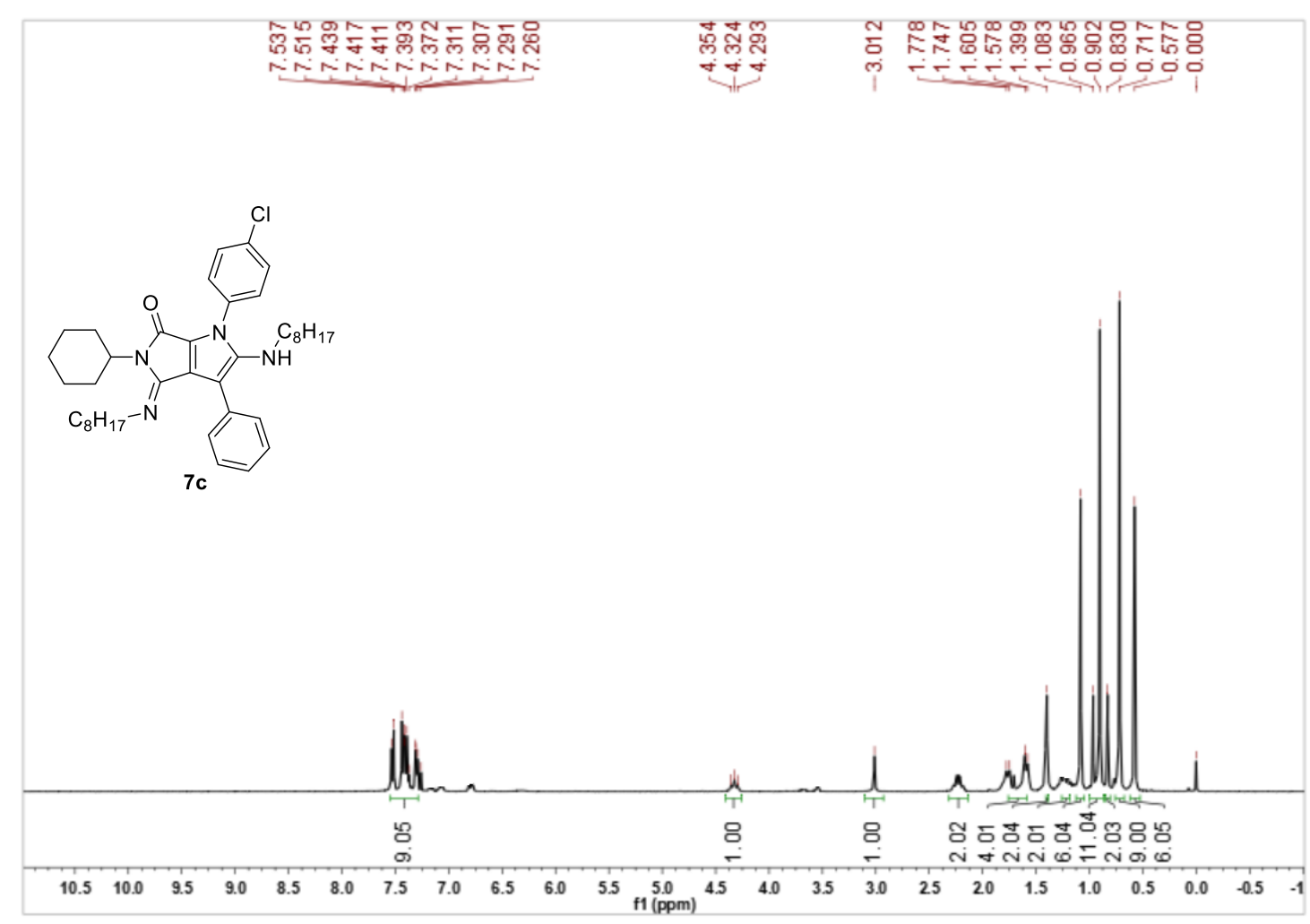

${ }^{1} \mathrm{H}$ NMR (400 MHz), $\mathrm{CDCl}_{3}$

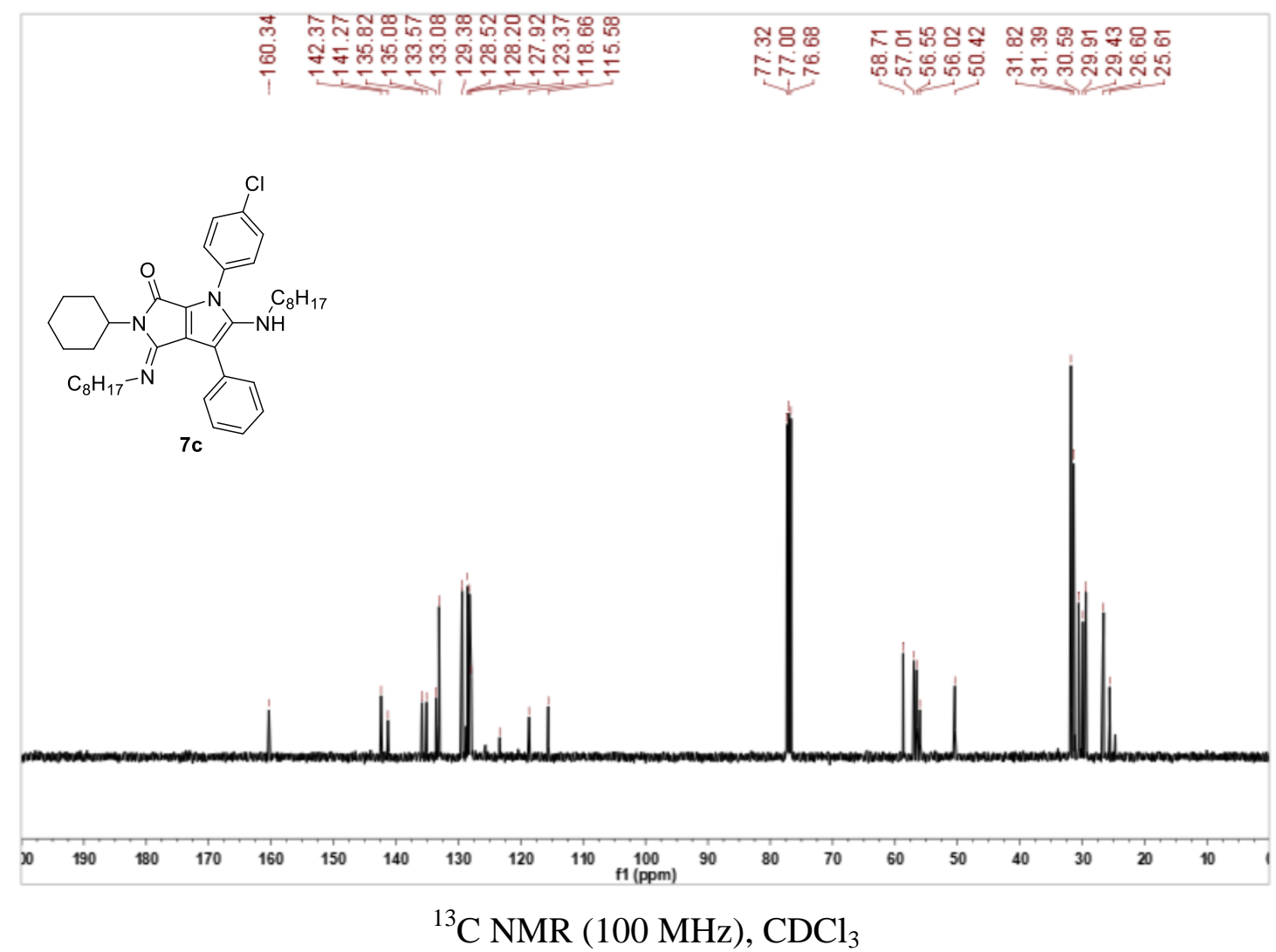




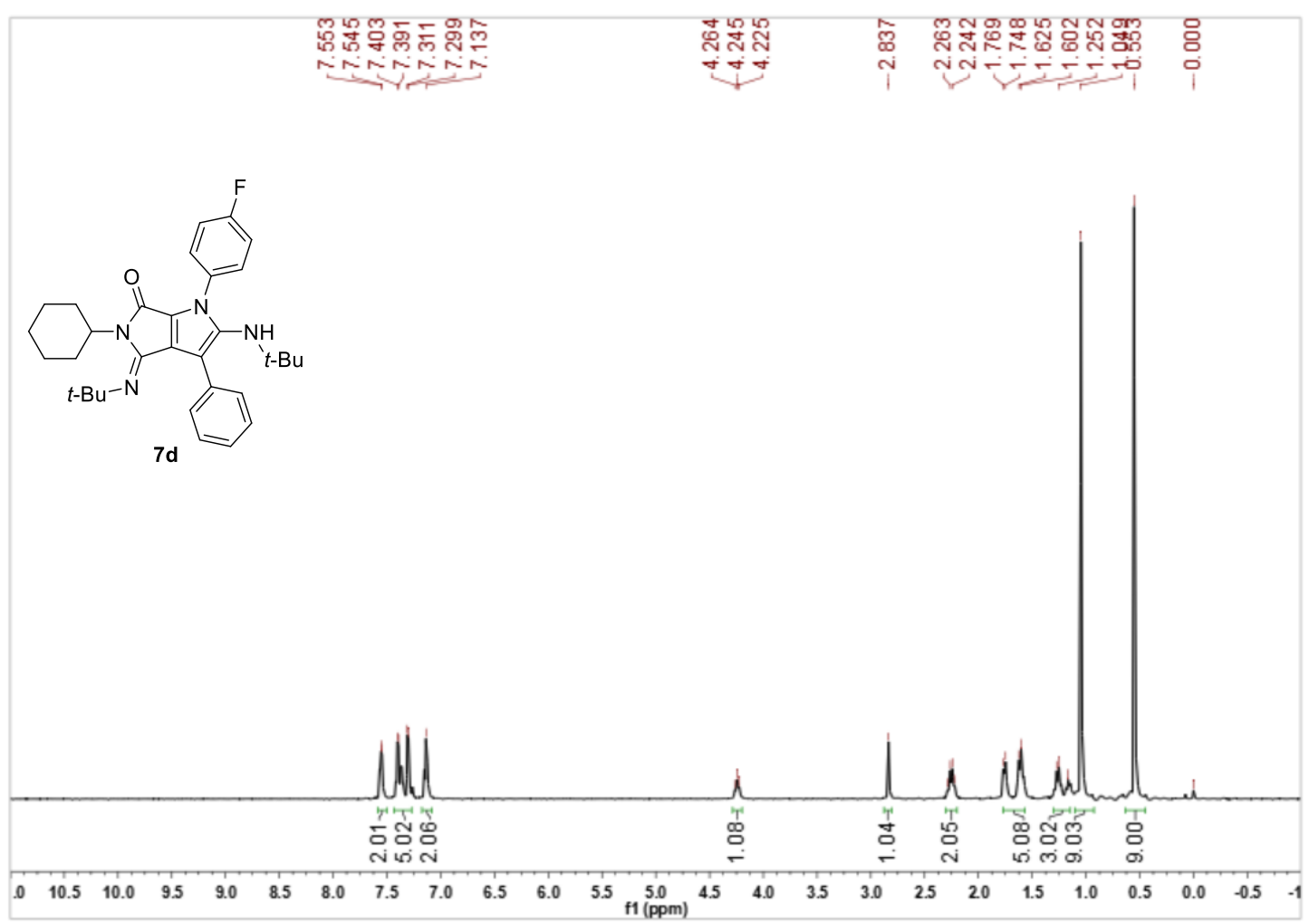

${ }^{1} \mathrm{H}$ NMR (400 MHz), $\mathrm{CDCl}_{3}$

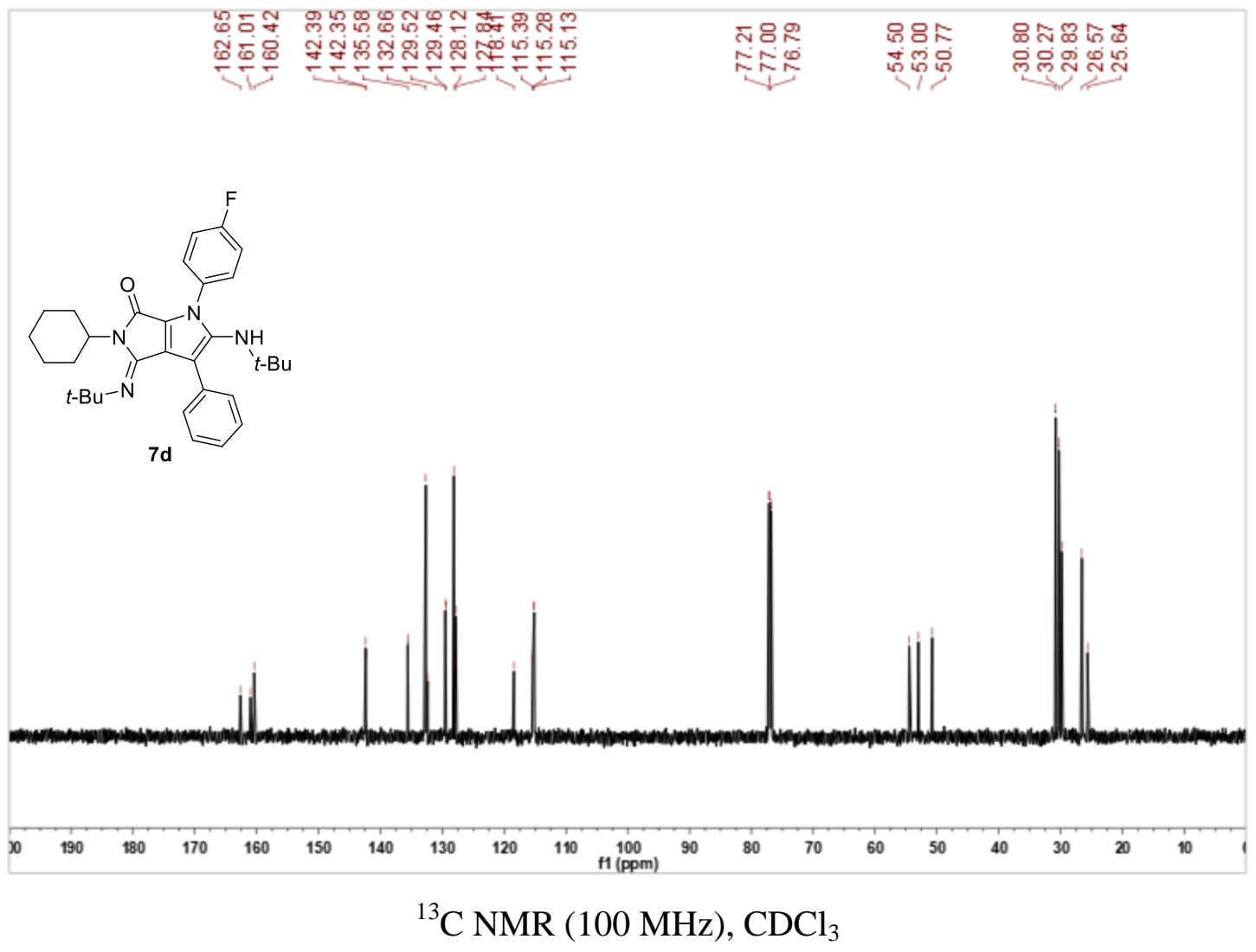




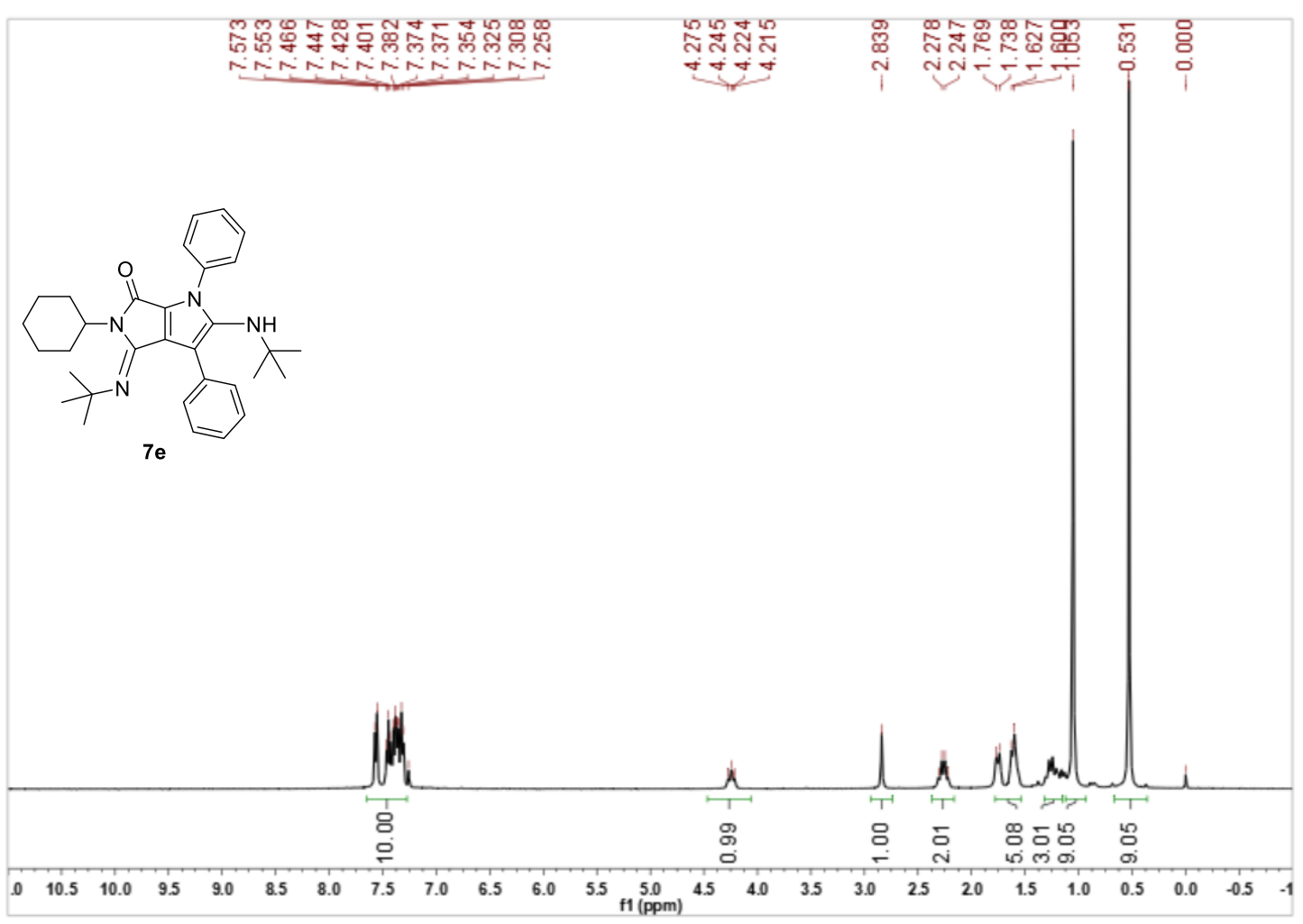

${ }^{1} \mathrm{H}$ NMR (400 MHz), $\mathrm{CDCl}_{3}$

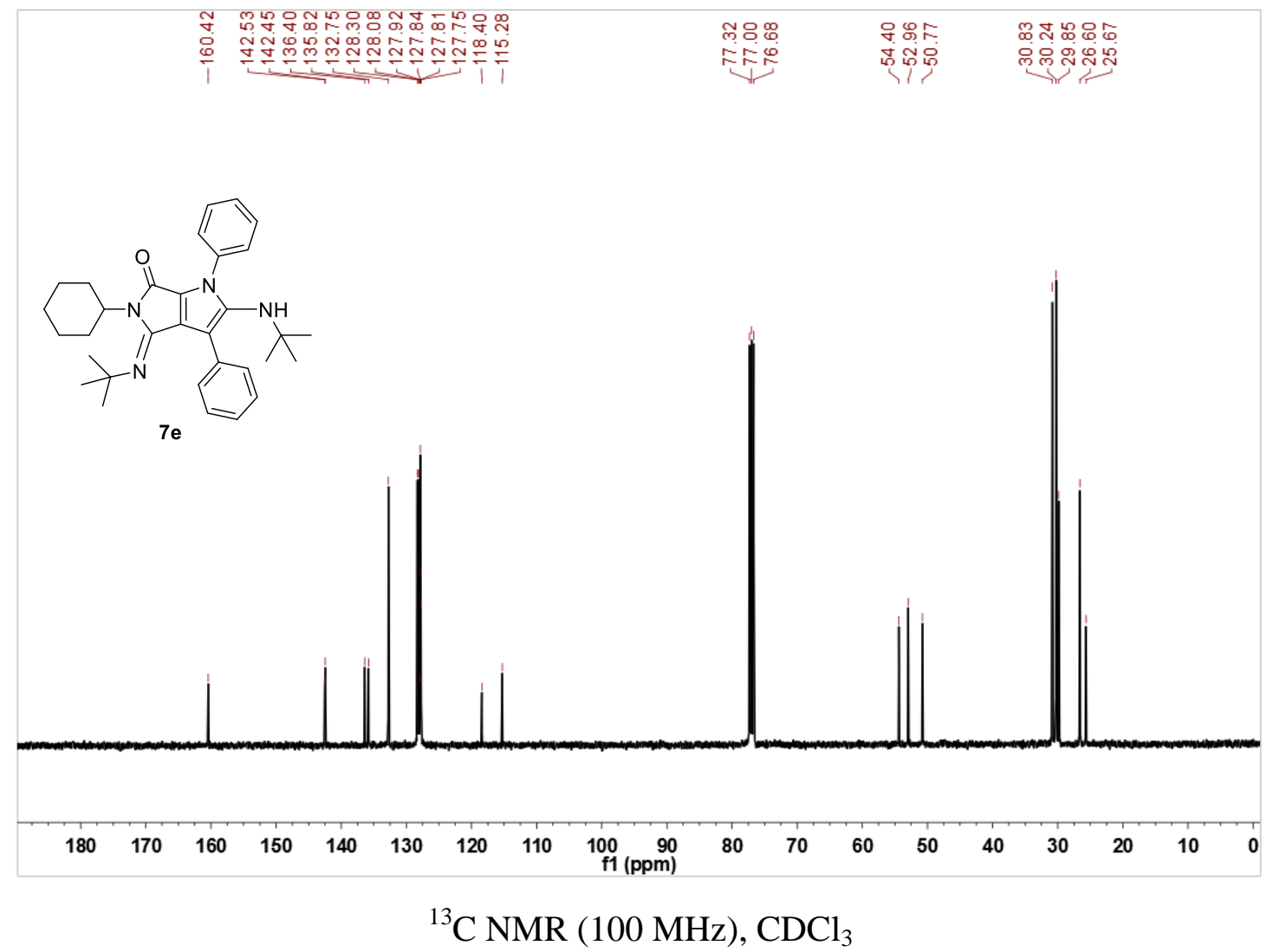




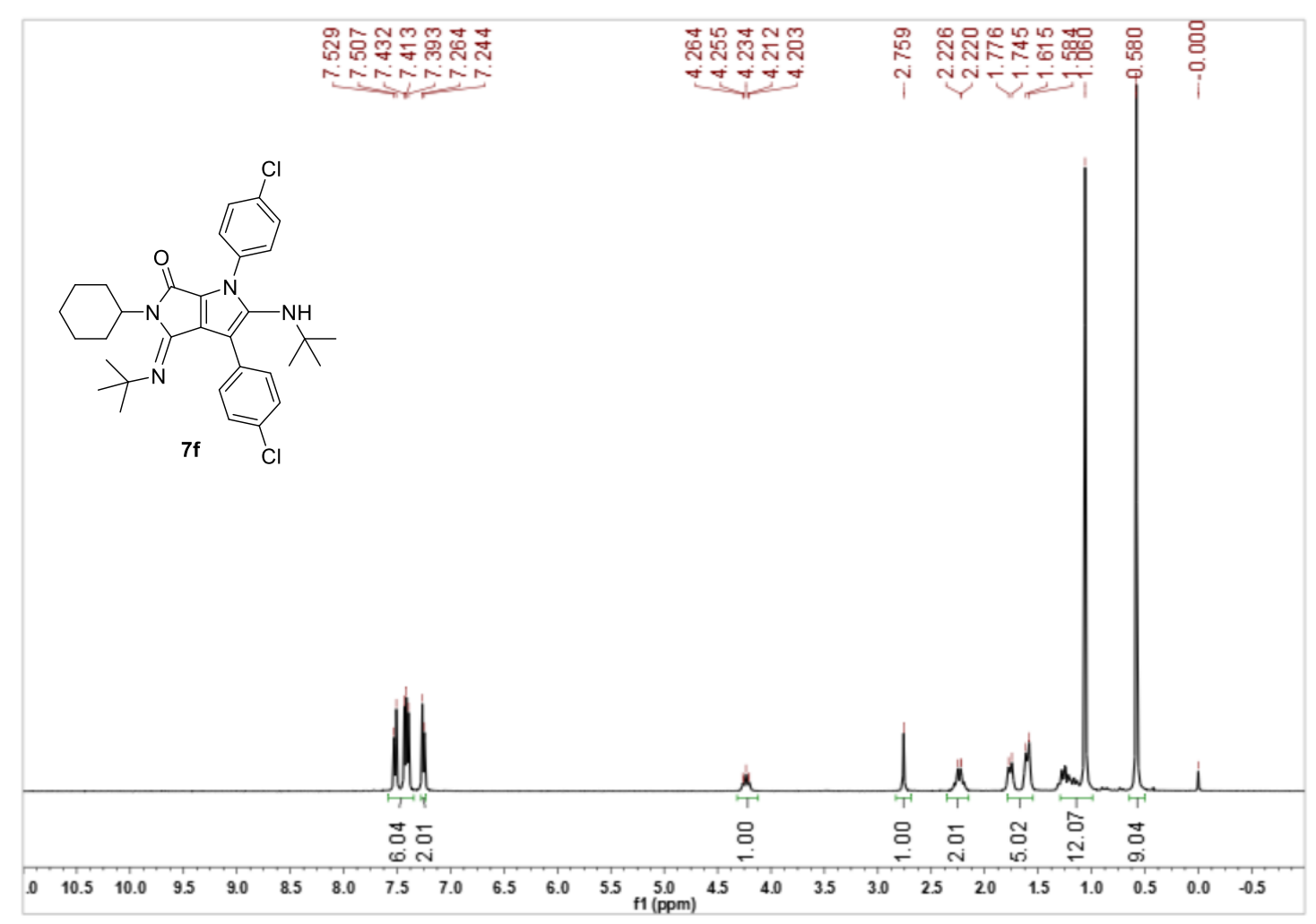

${ }^{1} \mathrm{H}$ NMR (400 MHz), $\mathrm{CDCl}_{3}$

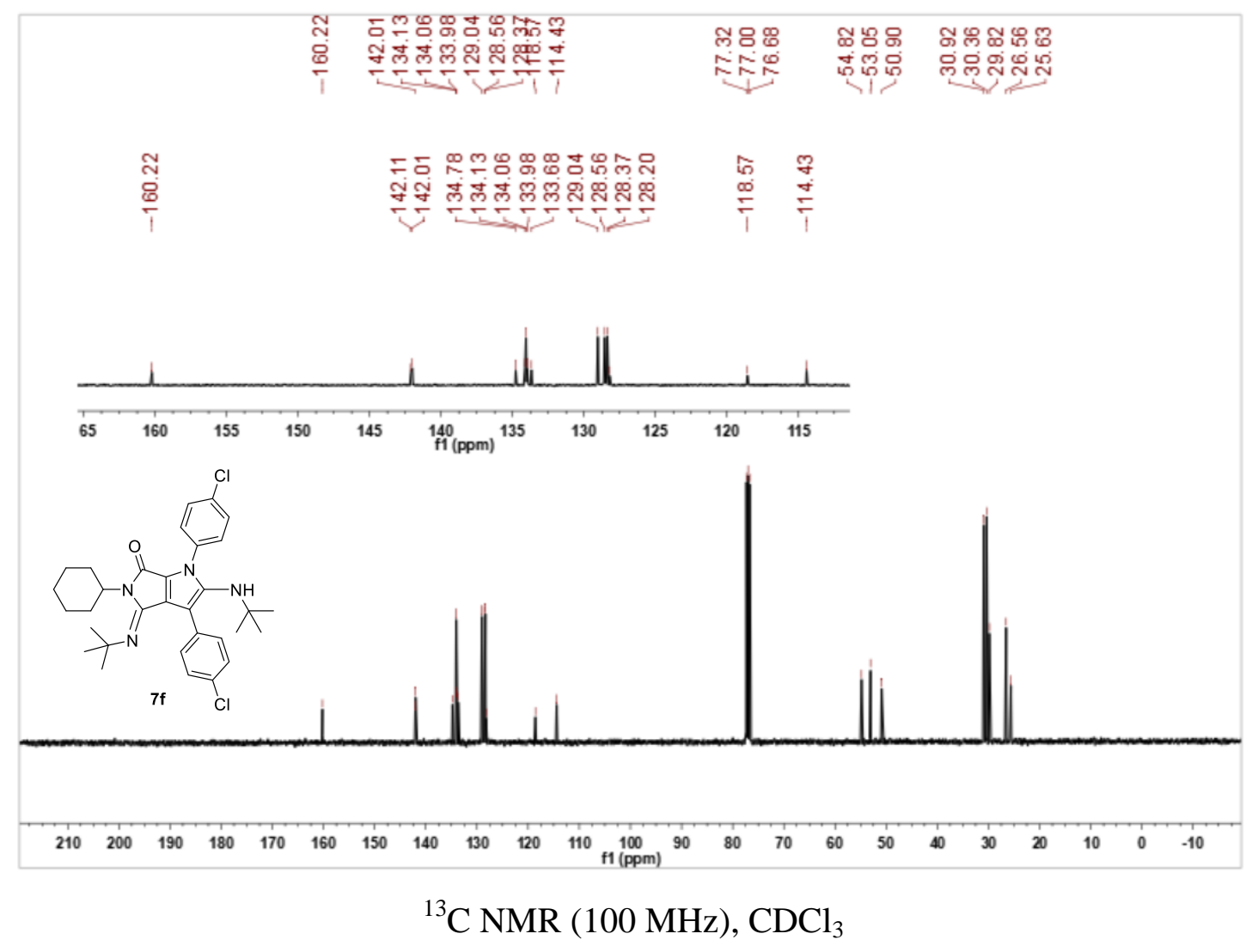




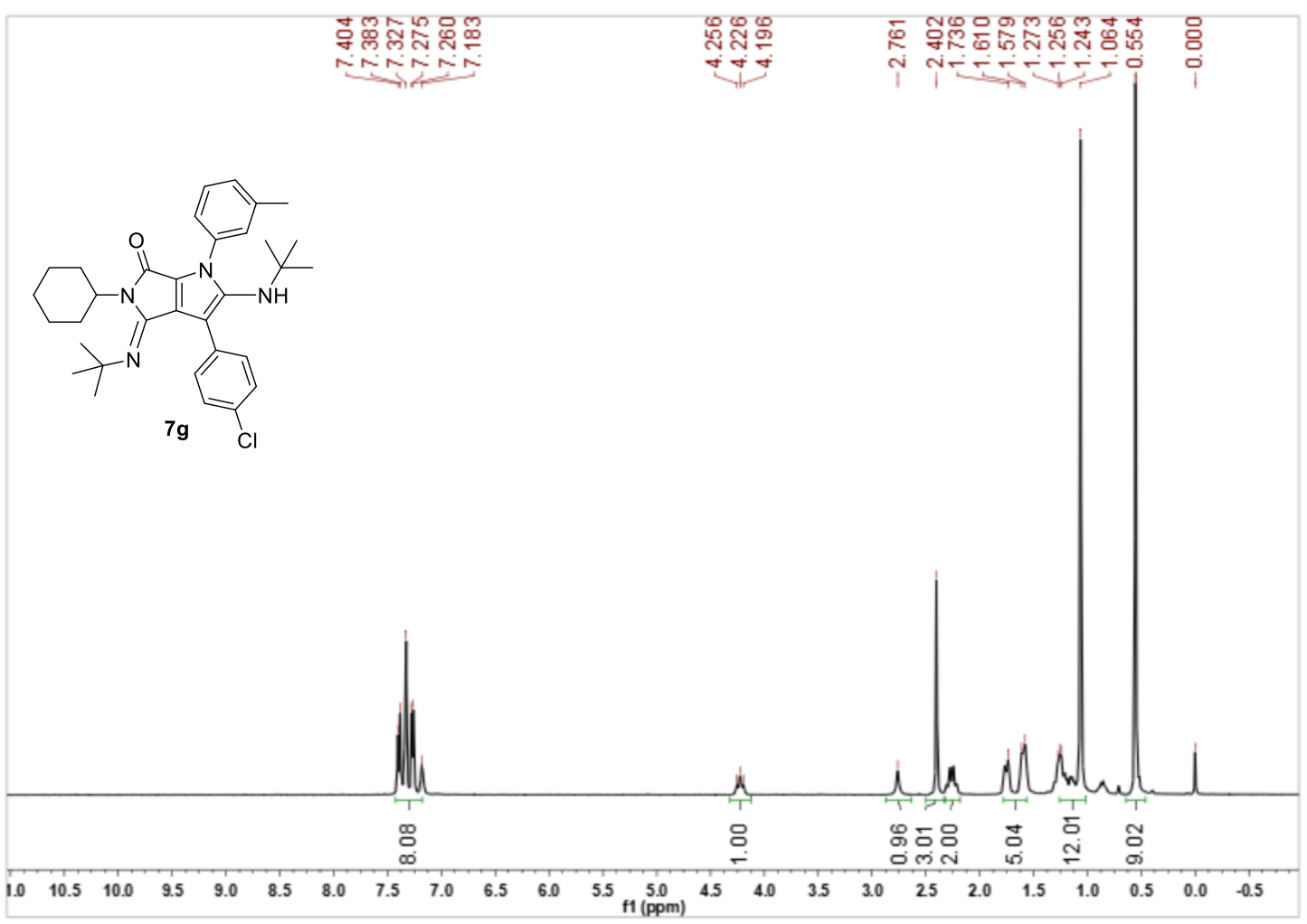

${ }^{1} \mathrm{H}$ NMR (400 MHz), $\mathrm{CDCl}_{3}$

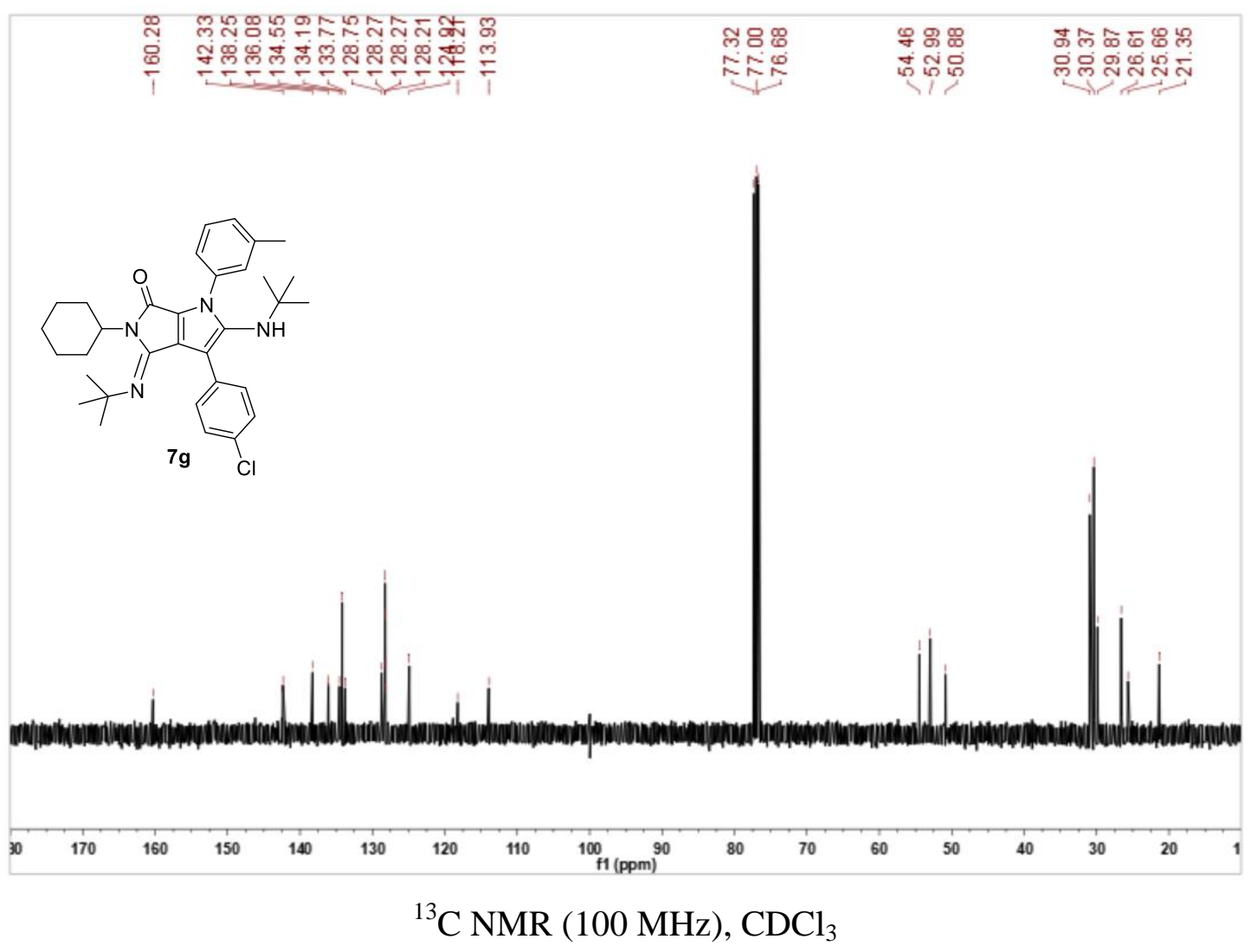




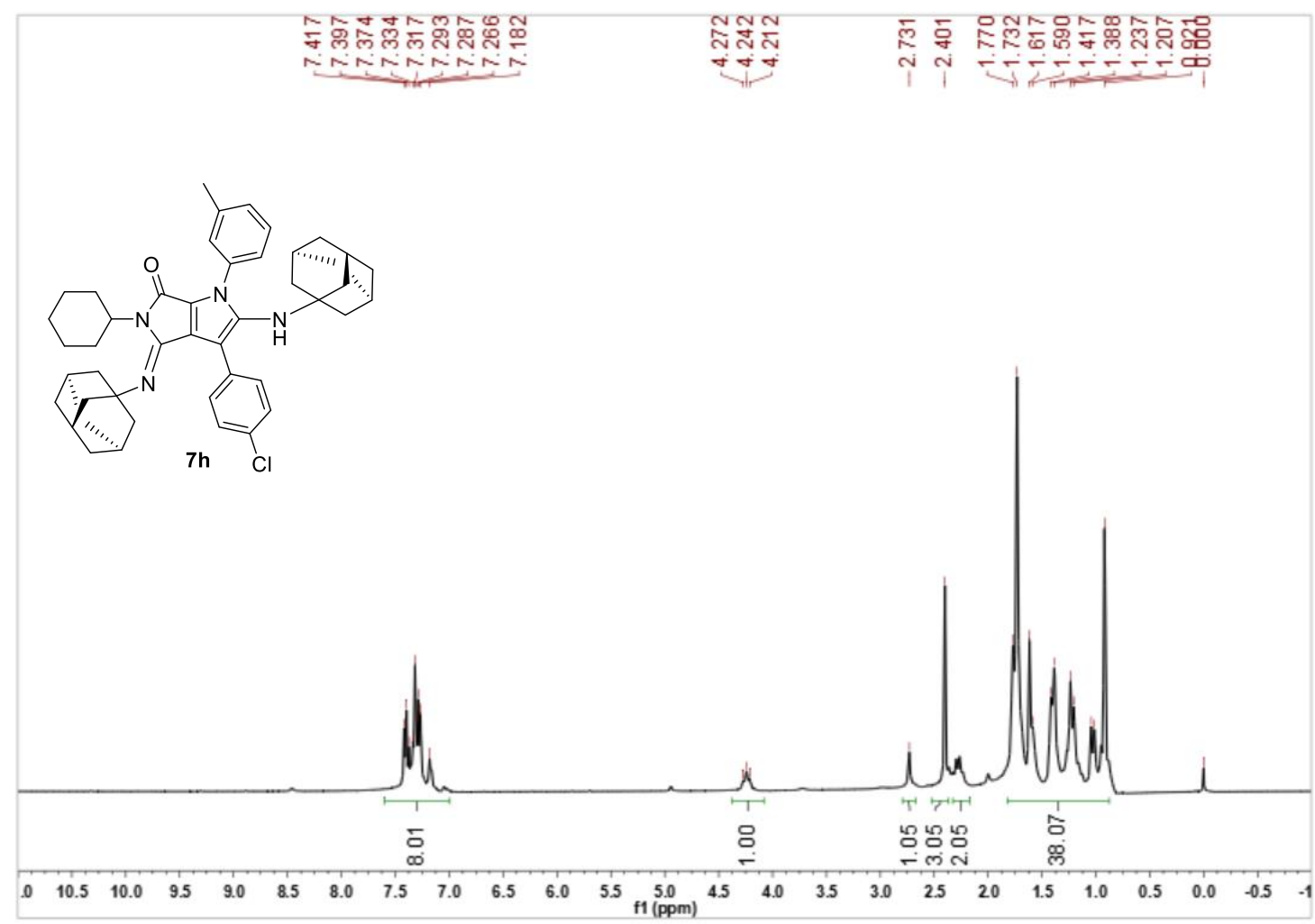

${ }^{1} \mathrm{H}$ NMR (400 MHz), $\mathrm{CDCl}_{3}$

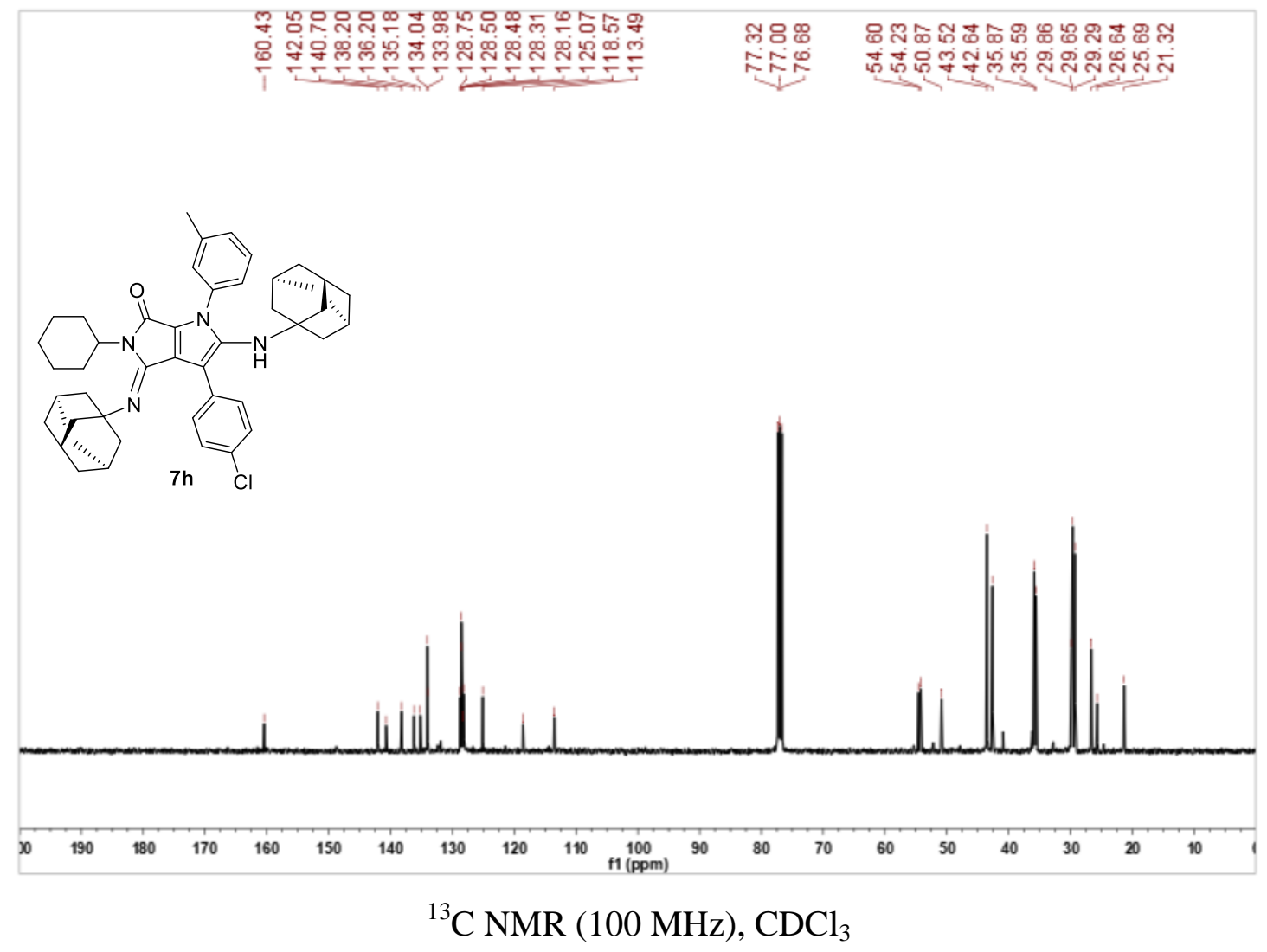




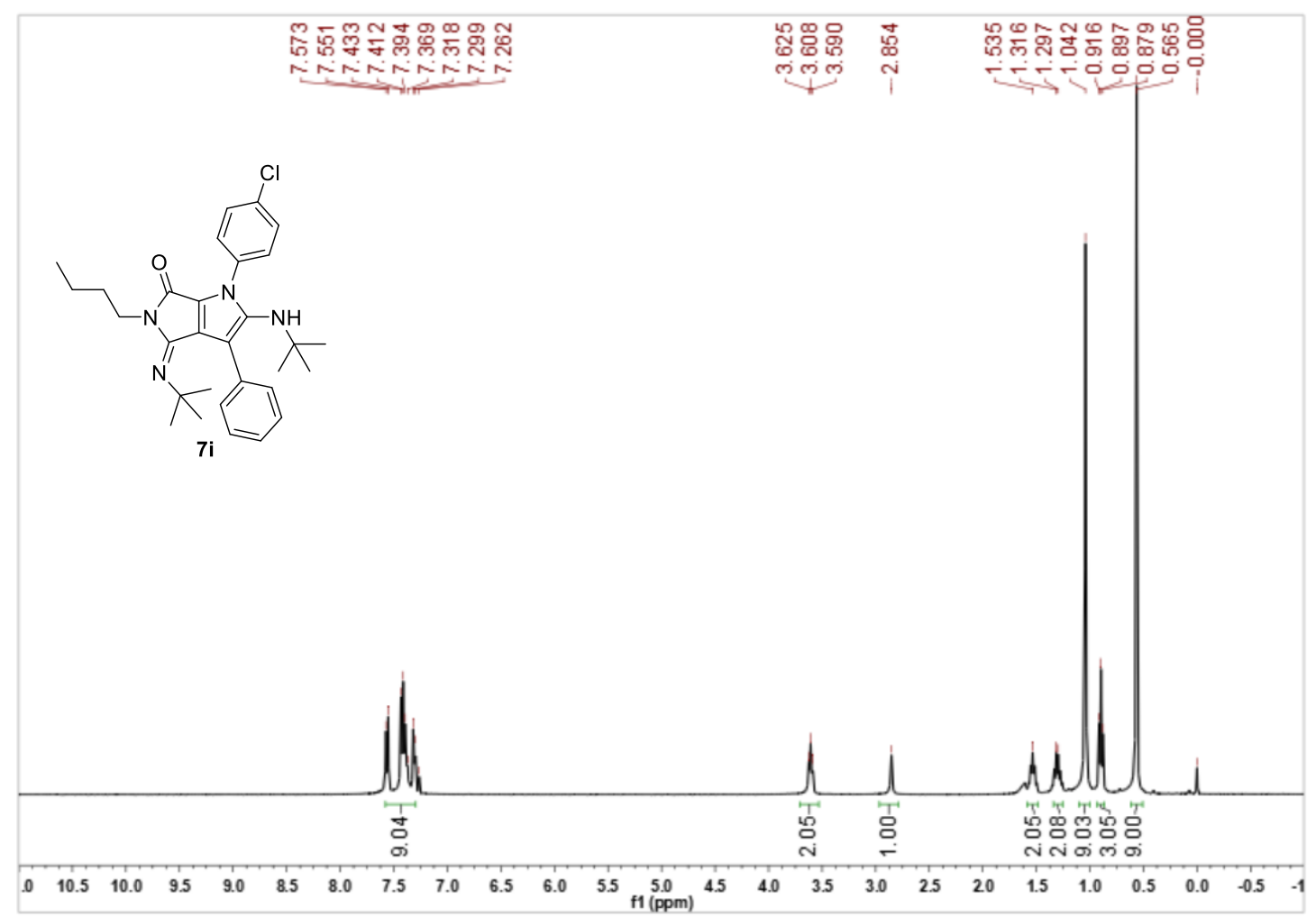

${ }^{1} \mathrm{H} \mathrm{NMR}(400 \mathrm{MHz}), \mathrm{CDCl}_{3}$

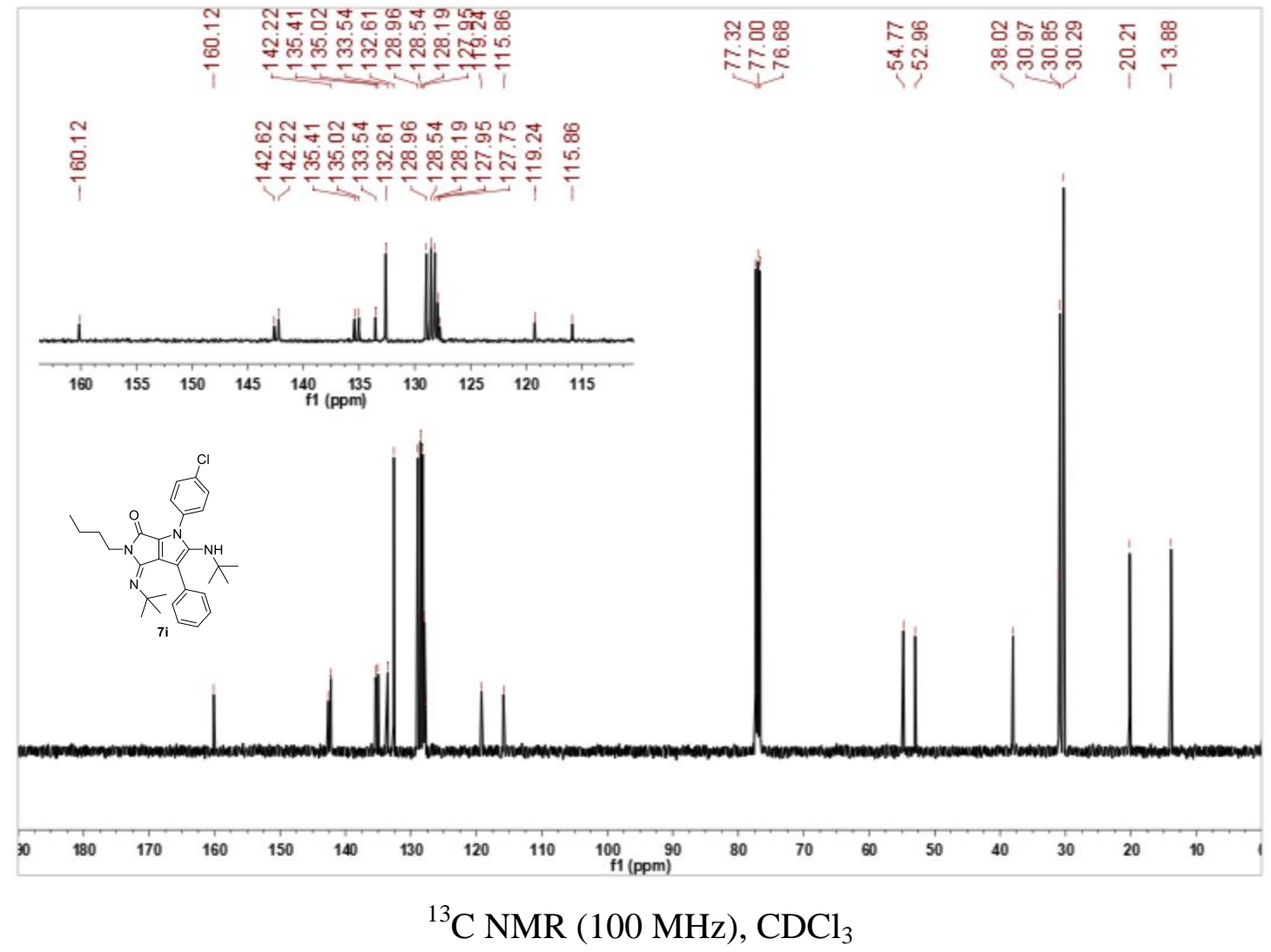




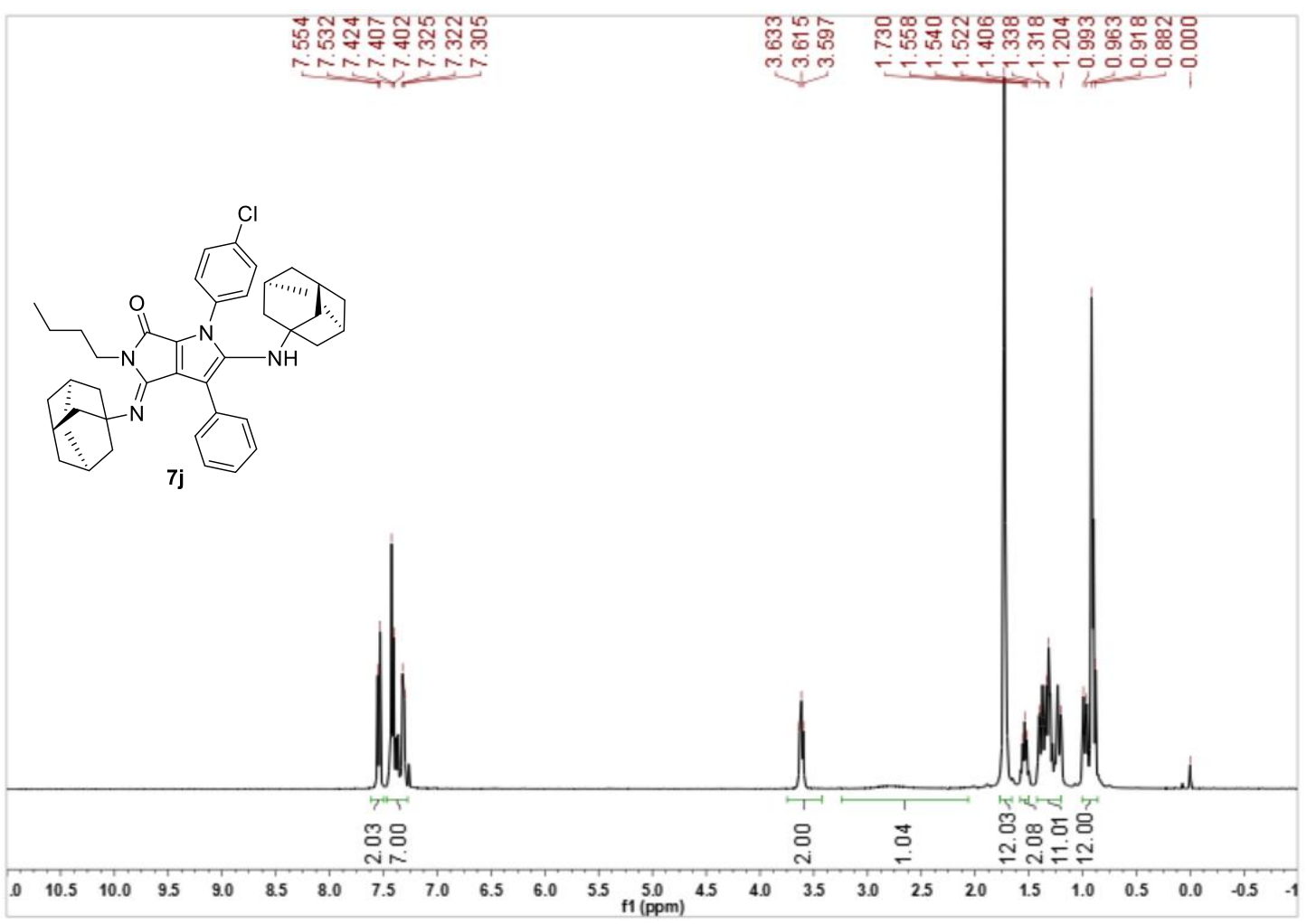

${ }^{1} \mathrm{H}$ NMR (400 MHz), $\mathrm{CDCl}_{3}$

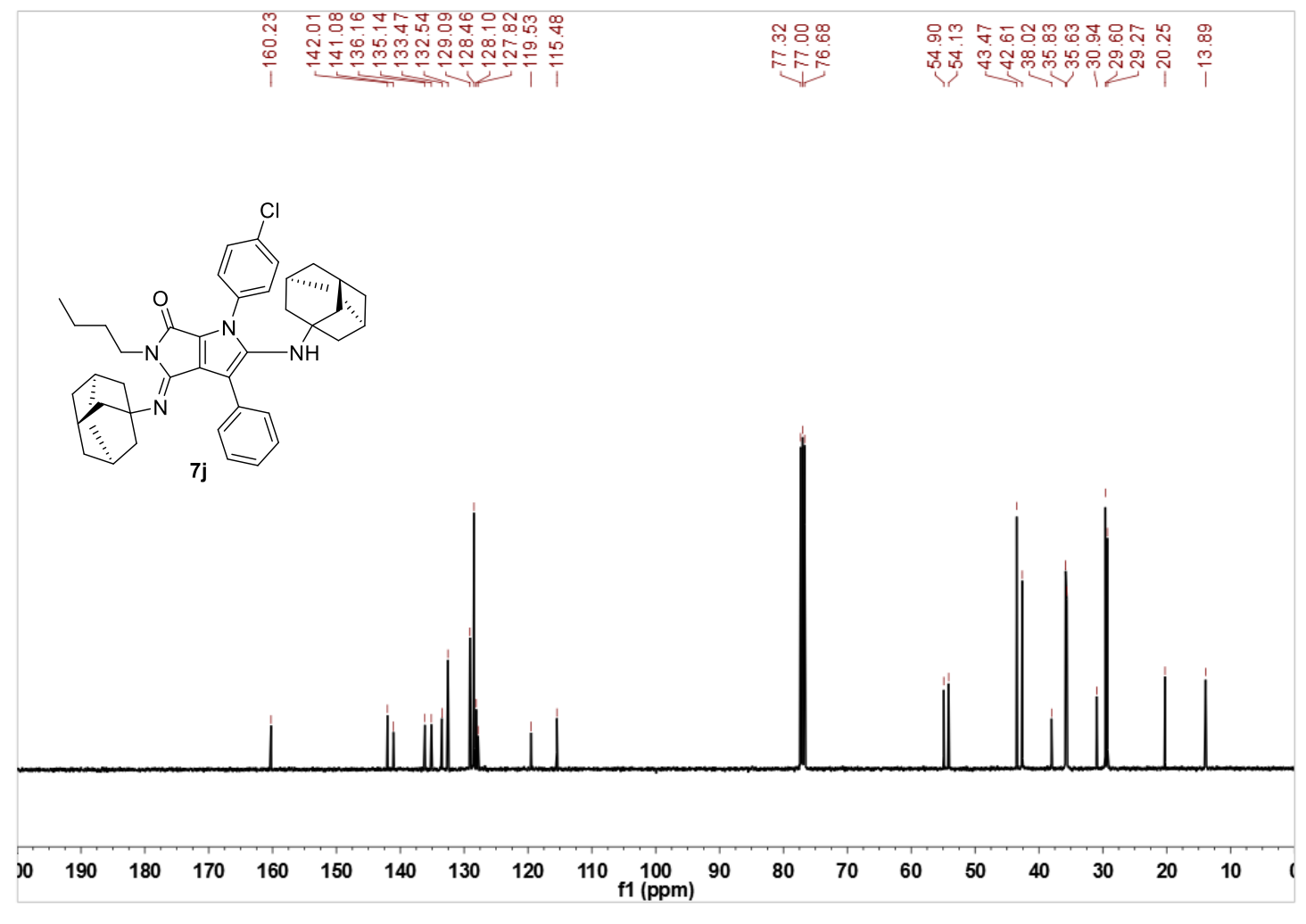

${ }^{13} \mathrm{C}$ NMR (100 MHz), $\mathrm{CDCl}_{3}$ 


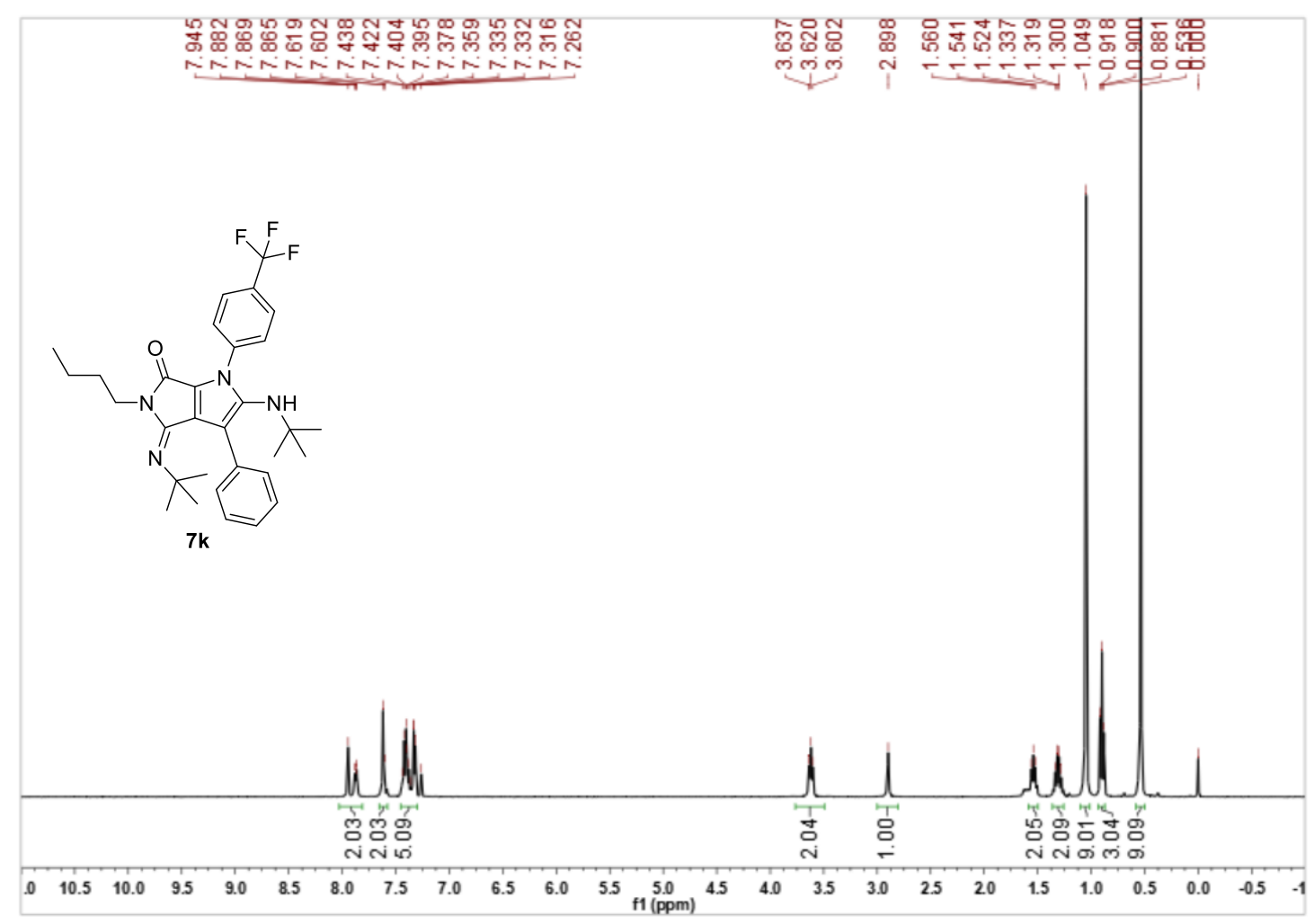

${ }^{1} \mathrm{H}$ NMR (400 MHz), $\mathrm{CDCl}_{3}$

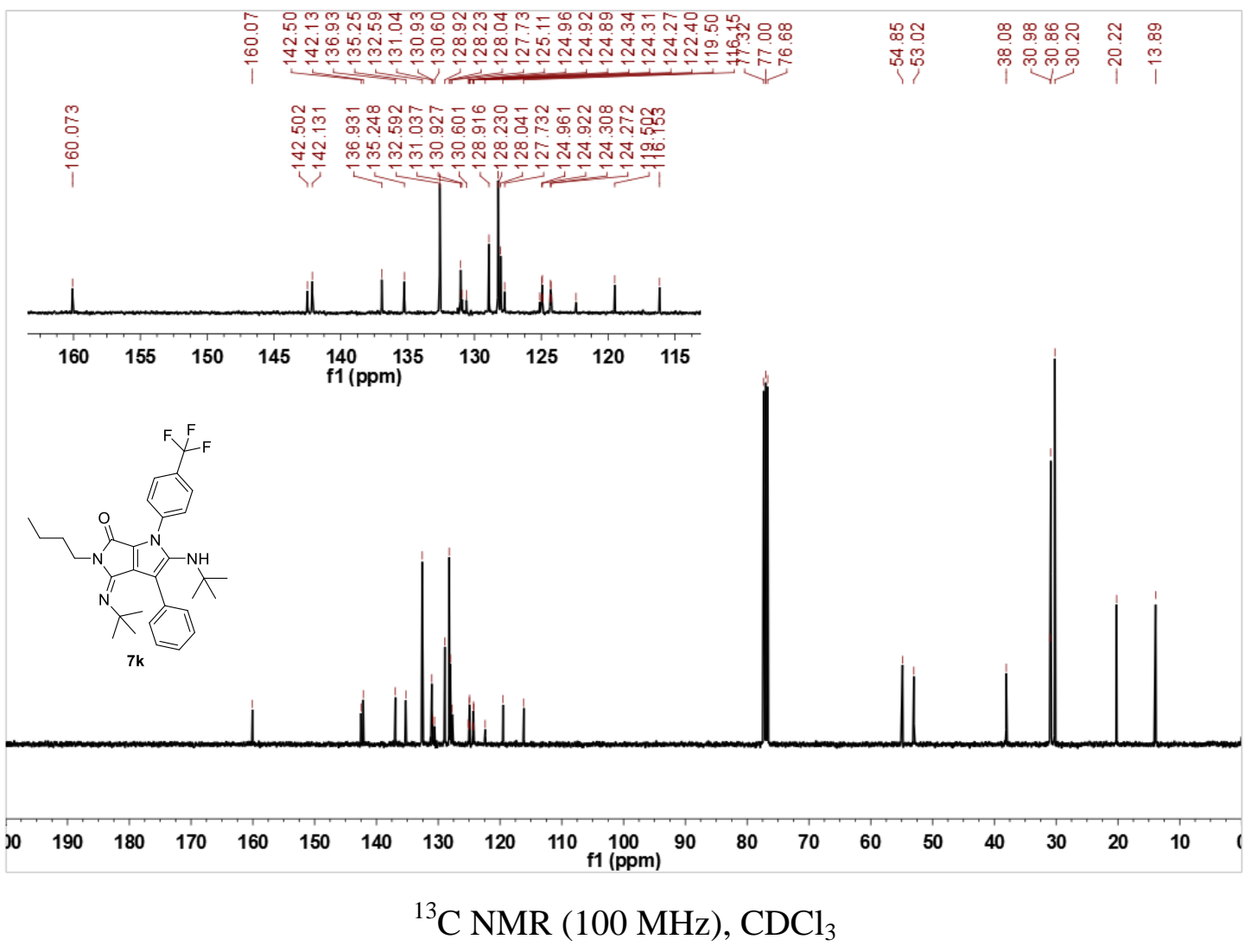

